\title{
Laserspektroskopische Untersuchungen zur Dynamik von ionischen Flüssigkeiten mit Hilfe molekularer Sonden
}

\author{
Dissertation \\ zur Erlangung des mathematisch- naturwissenschaftlichen \\ Doktorgrades \\ „Doctor rerum naturalium" \\ der Georg-August-Universität Göttingen
}

\author{
vorgelegt von \\ Peter William Lohse \\ aus Northeim
}

Göttingen 2010 
Referent: Prof. Dr. Jürgen Troe Korreferentin: PD Dr. Kawon Oum

Tag der mündlichen Prüfung: 12. Oktober 2010 
"Is there any conflict between science and religion?"

There is no conflict in the mind of God.

Henry Eyring 



\section{Danksagung}

Herrn Prof. Dr. Jürgen Troe möchte ich für die Bereitstellung des optimalen Arbeitsplatzes und für die freundliche Unterstützung und Beratung während der Doktorarbeit danken.

Besonderen Dank gilt PD Dr. Kawon Oum für die anregenden Diskussionen, die wesentlich zur Gestalt meiner Arbeit beitrugen. Ihr gebührt mein außerordentlicher Dank für die Einführung in die Welt der ionischen Flüssigkeiten. Danken möchte ich auch für die vielzählige Unterstützung die sie mir bei privaten Schwierigkeiten gewährt hat.

Herrn Prof. Dr. Thomas Lenzer danke ich ebenfalls für seine ständige Diskussionsbereitschaft.

Ein spezieller Dank gilt Reinhard Bürsing, dessen feinmechanischen Fähigkeiten wesentlich zum Gelingen dieser Arbeit beigetragen haben.

Mein Dank gilt auch allen Mitarbeitern und Mitarbeiterinnen der Werkstätten und des Instituts für ihre Hilfsbereitschaft.

Herrn Jens Schimpfhauser und Herrn Jürgen Bienert im Max-Planck-Institut für biophysikalische Chemie möchte ich für die Karl-Fischer-Titrationen der ionischen Flüssigkeiten danken.

Besonders danken möchte ich auch Herrn Dr. Hansgeorg Ernst von der BASF, der freundlicherweise die Carotinoide zur Verfügung gestellt hat.

Dank der lieben Kollegen wird mir die Zeit der Doktorarbeit unvergesslich bleiben. Daher möchte ich mich ganz besonders bei Matthäus, Florian, Heiko, Matthias, Arne, Lars, Elsa, Heinrich, Katrin, Sebastian, Yaxing und Alex bedanken.

Anatoli Maergoiz ist ein verflixt guter Gesprächspartner. Danke für die schönen Mittagspausen.

Für die nette Zusammenarbeit während ihrer Bachelorarbeit, möchte ich mich bei Steffen Schubert, Nils Bartels und Julia Kuhnt bedanken.

Meinen lieben Freunden Tobias, Moritz, Matthäus und Karsten danke ich für die schönen Jahre in Göttingen.

Der Unterstützung von meiner Familie verdanke ich alles.

Zum Schluss möchte ich meiner lieben Maren danken, die immer für mich da ist. 



\section{Inhaltsverzeichnis}

1 Einleitung 1

2 Mikroskopische Polarität von ionischen Flüssigkeiten: Spektroskopische Methoden

2.1 UV/Vis- und Fluoreszenz-Spektroskopie solvatochromer Farbstoffe . . . . . . 6 2.1.1 Reichardts Farbstoff . . . . . . . . . . . . . . . . . . 7

2.1.2 Kamlet-Abboud-Taft-Parameter ................ 8

2.2 ESR-Spektroskopie von Spinprobes in ionischen Flüssigkeiten . . . . . . . . . 10

2.3 Ultraschnelle Laserspektroskopie . . . . . . . . . . . . . . . . . . . . . . . . . . 11

3 Experimentelle Technik 15

3.1 Prinzip der Femtosekundenspektroskopie . . . . . . . . . . . . . . . . . . . 16

3.2 Breitband Pump-Superkontinuum-Probe-Spektroskopie . . . . . . . . . . . 17

3.2.1 Das Hurricane-System . . . . . . . . . . . . . . . . . . . . . . . . . . 18

3.2.2 Erzeugung der Anregungspulse . . . . . . . . . . . . . . . . . 20 20

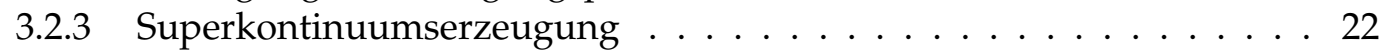

3.2 .4 Chirp-Korrektur . . . . . . . . . . . . . . . . 23

3.3 Ultraschnelles transientes Absorptionsexperiment . . . . . . . . . . . . 24

3.3 .1 Das Tsunami-System . . . . . . . . . . . . . . . . . . 26

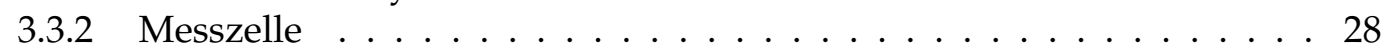

3.3 .3 Datenaufnahme . . . . . . . . . . . . . . . . . . 29

3.4 UV/Vis-spektroskopische Untersuchungen: Solvatochromie . . . . . . . . . 30

3.5 Chemikalien . . . . . . . . . . . . . . . . . . . . . 31

4 Apocarotinoide zur Untersuchung lokaler Wechselwirkungen in ionischen Flüssigkeiten 33

4.1 Ultraschnelle intramolekulare Prozesse von Apocarotinoiden . . . . . . . . . 35

4.2 Globale Analyse der PSCP-Daten . . . . . . . . . . . . . . . . . . 36

4.3 Experimentelle Ergebnisse für 12'-Apo- $\beta$-carotin-12'-säure . . . . . . . . . . . 39

4.4 Experimentelle Ergebnisse für 12 '-Apo- $\beta$-carotin-12'-al . . . . . . . . . . 48

4.5 Diskussion . . . . . . . . . . . . . . . . . . 554

5 Mikroskopische Eigenschaften von ionischen Flüssigkeiten nach Zugabe von Acetonitril

5.1 Kinetisches Modell zur Interpretation der transienten Absoprtsionsmessungen . . . . . . . . . . . . . . . . . . 60

5.2 Ergebnisse für die Mischungen von $\left[\mathrm{C}_{6} \mathrm{mim}\right]^{+}\left[\mathrm{Tf}_{2} \mathrm{~N}\right]^{-}$mit Acetonitril . . . 63

5.2.1 Stationäre Absorptionsspektroskopie . . . . . . . . . . . . . . 64 . . 64

5.2.2 Pump-Superkontinuum Probe (PSCP)-Spektroskopie . . . . . . . . . . 666 66

5.2.3 Transiente Absorptions- (TA) Spektroskopie . . . . . . . . . . . . . . 78 
5.3 Ergebnisse für die Mischung $\left[\mathrm{C}_{2} \mathrm{mim}\right]^{+}\left[\mathrm{EtSO}_{4}\right]^{-} /$Acetonitril $\ldots . . . . . .83$

5.3.1 Ergebnisse des transienten Absorptionsexperiments . . . . . . . . . . . 83

5.3.2 Solvatochrome Untersuchungen . . . . . . . . . . . . . . . 887

5.4 Diskussion . . . . . . . . . . . . . . . . . . . 88

6 Indolinbasierte Farbstoffe für Solarzellen 99

6.1 Prinzip der DSSC . . . . . . . . . . . . . . . . . . . . . . . 100

6.2 Sensibilatoren und Elektrolyte . . . . . . . . . . . . . . . . . . . . . 101

6.3 Ultraschnelle intramolekulare Prozesse von indolinbasierten Farbstoffen . . .103

6.4 Experimentelle Ergebnisse für D102, D131, D149 und D205 . . . . . . . . . . 105

6.4.1 Stationäre Absorptionsspektroskopie . . . . . . . . . . . . . . . 105

6.4.2 Transiente Absorptions- (TA) Spektroskopie . . . . . . . . . . . . . . 107

6.4.3 Pump-Superkontinuum-Probe-Spektroskopie . . . . . . . . . . . . 110

6.5 Diskussion . . . . . . . . . . . . . . . . . . . . . . . . 1117 


\section{Zusammenfassung}

Ionische Flüssigkeiten wurden in dieser Arbeit mittels transienter Absorptionsspektroskopie untersucht. Die Relaxationsprozesse molekularer Sonden nach Photoanregung dienten als Indikator, um die mikroskopische Umgebung in ionischen Flüssigkeiten und deren Mischungen mit organischen Lösungsmitteln verstehen zu können. Es werden zwei ultraschnelle Pump-Probe-Techniken angewandt. Zum einen die Pump-SuperkontinuumProbe- (PSCP) Spektroskopie im Bereich von 350 bis $770 \mathrm{~nm}$ und zum anderen ZweifarbenPump-Probe-Messungen im nahen IR-Bereich bei $860 \mathrm{~nm}$.

Die Einführung einer Carbonylfunktion in ein Carotinoid führt zu einer leistungsstarken Sonde für die Untersuchung der Polarität und Solvatationsdynamik ionischer Flüssigkeiten. Die molekularen Sonden 12'-Apo- $\beta$-carotin-12'-säure und 12'-Apo- $\beta$-carotin-12'-al wurden bei den PSCP-Messungen mit $480 \mathrm{~nm}$ in den $\mathrm{S}_{2}$-Zustand angeregt. Durch schnelle innere Konversion mit etwa 100 fs wurde der $\mathrm{S}_{1} /$ ICT-Zustand mit intramolekularem Charge-Transfer-Charakter besetzt. Dieser Zustand besitzt eine von der Polarität des Lösungsmittel abhängige Lebensdauer und zeigt eine spezifische spektrale Verschiebung der Absorptionsbanden mit der Zeit.

Eine andere Herangehensweise zur Charakterisierung der mikroskopischen Eigenschaften von ionischen Flüssigkeiten ist das Mischen mit organischen Lösungsmitteln. Für die Messungen dieser binären Mischungen wurde die molekulare Sonde 12'-Apo- $\beta$-carotin12 '-säure verwendet. Es konnte gezeigt werden, dass sich bei den Messungen in $\left[\mathrm{C}_{6} \mathrm{~m}\right.$ $\mathrm{im}]^{+}\left[\mathrm{Tf}_{2} \mathrm{~N}\right]^{-}$die Polarität der mikroskopische Umgebung dieser Sonde bis hin zu hohen Verdünnungen auf einer kurzen Zeitskala nach einer elektronischen Anregung kaum ändert und somit der Polarität in der reinen ionischen Flüssigkeit gleicht.

Ein Anwendungsgebiet der ionischen Flüssigkeiten sind Farbstoffsolarzellen. Hierbei wird das Licht durch organische Moleküle und nicht durch einen Halbleiter absorbiert. Mit ionischen Flüssigkeiten als Elektrolyten wurden bereits recht hohe Wirkungsgrade erreicht, doch ist über die Wechselwirkung zwischen den verwendeten Farbstoffen und dieser Art von Lösungsmitteln nichts genaues bekannt. In dieser Arbeit wurde eine Klasse von indolinbasierten Farbstoffen untersucht. Es konnte gezeigt werden, dass der Farbstoff D205 in protischen Lösungsmitteln die höchste der gemessen Lebensdauern des angeregten $\mathrm{Zu}$ stands aufweist. Dieser Farbstoff ist unter der Klasse der indolinbasierten Sensibilisatoren von Solarzellen derjenige mit dem höchsten Wirkungsgrad. 



\section{Abkürzungen}

$12^{\prime} \mathrm{CA}$

12'TAC

$\left[\mathrm{C}_{2} \mathrm{mim}\right]^{+}\left[\mathrm{N}(\mathrm{CN})_{2}\right]^{-}$

$\left[\mathrm{C}_{2} \mathrm{mim}\right]^{+}\left[\mathrm{EtSO}_{4}\right]^{-}$

$\left[\mathrm{C}_{4} \mathrm{mim}\right]^{+}\left[\mathrm{Tf}_{2} \mathrm{~N}\right]^{-}$

$\left[\mathrm{C}_{4} \mathrm{mim}\right]^{+}\left[\mathrm{B}(\mathrm{CN})_{4}\right]^{-}$

$\left[\mathrm{C}_{4} \mathrm{mmim}\right]^{+}\left[\mathrm{Tf}_{2} \mathrm{~N}\right]^{-}$

$\left[\mathrm{C}_{6} \mathrm{mim}\right]^{+}\left[\mathrm{Tf}_{2} \mathrm{~N}\right]^{-}$

D102

D131

D149

D205

ESA

GSB

ICT

IL

PSCP

SE

TA

THF

TMS
12'-Apo- $\beta$-carotin-12'-säure

12 '-Apo- $\beta$-carotin-12'-al

1-N-Ethyl-3-N-methyl-imidazolium-dicyanamid

1-N-Ethyl-3-N-methylimidazoliumethylsulfat

1-N-Butyl-3-N-methyl-imidazolium-

bis(trifluoromethylsulfonyl)imid

1-N-Butyl-3-N-methyl-imidazolium-tetracyanoborat

1-N-Hexyl-2,3-N-dimethyl-imidazolium-

bis(trifluoromethylsulfonyl)imid

1-N-Hexyl-3-N-methyl-imidazolium-

bis(trifluoromethylsulfonyl)imid

indolinbasierte Farbstoffe,

chemische Strukturformeln siehe S. 32

Excited-State-Absorption

Ground-State-Bleach

Intramolecular-Charge-Transfer

ionische Flüssigkeit

Pump-Supercontinuum-Probe

stimulierte Emission

transiente Absorption

Tetrahydrofuran

Tetramethylsilan 



\section{Kapitel 1}

\section{Einleitung}

Ionische Flüssigkeiten (ILs) stellen außergewöhnliche Lösungsmittelmedien dar, welche gänzlich aus Ionen bestehen. Es sind organische Salze, die Schmelzpunkte unterhalb von $100{ }^{\circ} \mathrm{C}$ besitzen. Für viele Verbindungen zeigen sie hervorragende Lösungseigenschaften und verhalten sich vergleichbar mit mittelpolaren Lösungsmitteln, wie kurzkettigen Alkoholen. Allerdings haben ILs im Vergleich zu organischen Lösungsmitteln andere makroskopische Eigenschaften. Die Viskositäten und Siedepunkte sind wesentlich höher, was für ein stark geordnetes Ionennetzwerk in diesen Medien spricht. Ein Vorteil beim Gebrauch von ILs ist die große Anzahl möglicher Kombinationen von Kationen und Anionen, wodurch es möglich ist, Lösungsmittel mit gewünschten Eigenschaften zu kreieren. $1+3$

In Abbildung 1.1 sind Beispiele einiger gebräuchlicher Kationen und Anionen zum Aufbau ionischer Flüssigkeiten zu finden. Die Kationen lassen sich allgemein in zwei strukturell unterschiedliche Arten unterteilen:

i) relativ kleine, starre Ionen mit Ringstruktur

ii) große und flexible Ionen, wie Alkylammonium und Alkylphosphonium

Als Anionen kommen kleine anorganische Ionen, wie die Halogenide, aber auch größere organische Verbindungen in Frage. Einige der Verbindungen basierend auf Imidazolium und Pyridinium sind sogar bis $-80{ }^{\circ} \mathrm{C}$ flüssig, doch liegen die Schmelzpunkte der meisten ionischen Flüssigkeiten sehr viel höher. $\frac{455}{}$ Ionische Flüssigkeiten haben einen geringen Dampfdruck, es gibt allerdings Ausnahmen. So ist es z.B. möglich, einige imidazoliumbasierte ionische Flüssigkeiten zu destillieren. ${ }^{677}$ Des Weiteren zeichnen sie sich durch ei- 
ne außergewöhnlich hohe thermische Stabilität aus. Eine chemische Zersetzung tritt erst bei hohen Temperaturen auf, bis zu $450{ }^{\circ} \mathrm{C}$ (bei 1-Hexyl-4-(4-methylpiperidino)pyridinbis(trifluoromethylsulfonyl)imid) können dabei erreicht werden. ${ }^{[5] 9}$ Die Struktur der ILs nimmt einen wesentlichen Einfluss auf deren Viskositäten, die ca. 10000 cP (z. B. EcoEng $^{T M}$-500) erreichen können. ${ }^{25]}$ Ionische Flüssigkeiten mit EcoEng ${ }^{T M}$ als Abkürzung basieren auf Sulfaten als Anionen, EcoEng ${ }^{T M}-500$ hat Methylsulfat als Anion. Die Viskositäten der für diese Arbeit relevanten Ils liegen in der Größenordnung von 10 bis $100 \mathrm{cp}$. Die Mischbarkeit mit anderen Flüssigkeiten ist ebenfalls eine wichtige Eigenschaft der ionischen Flüssigkeiten. Obwohl es solche gibt, die sich mit Wasser nicht mischen lassen, sind alle ILs hygroskopisch. Hierbei muss man sich verdeutlichen, dass schon kleine Mengen Wasser eine große Verunreinigung darstellen können, denn aufgrund der hohen molaren Masse der ILs bedeutet eine kleine Masse an Wasser bereits einen hohen Molenbruch. Wasserverunreinigungen können die physikalischen Eigenschaften der ILs beeinflussen. Für die in dieser Arbeit relevanten Messungen spielen Wasserverunreinigungen durch Hygroskopie nur eine untergeordnete Rolle, wie früher gezeigt werden konnte. ${ }^{10}$
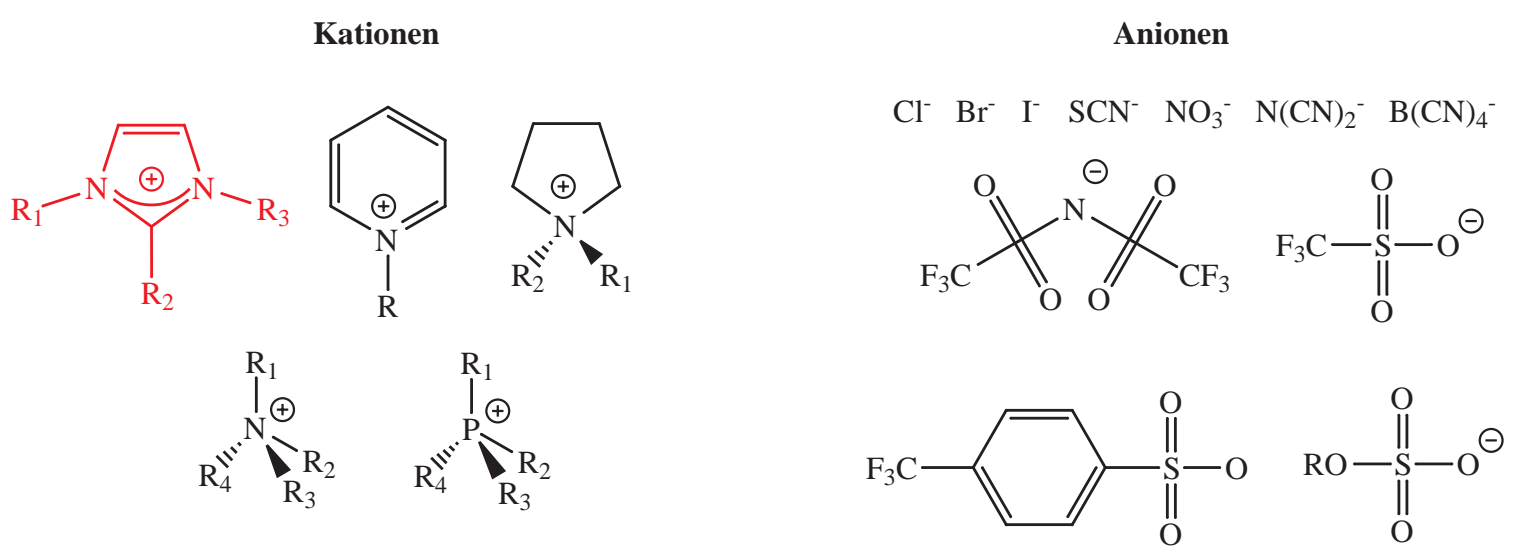

Abbildung 1.1: Chemische Strukturformeln ionischer Flüssigkeiten. Rot: Die für die vorliegende Arbeit zentrale imidazoliumbasierte ionische Flüssigkeit.

Die Untersuchung der mikroskopischen Wechselwirkungen in ILs ist erforderlich, um ihre Eigenschaften vorhersagen zu können. In dieser Arbeit werden Solvatationsprozesse von ionischen Flüssigkeiten auf einer ultrakurzen Zeitskala (wenige Femtosekunden bis zu einigen hundert Pikosekunden) untersucht. Die Lösungsmittelstruktur in der Umgebung einer gelösten Substanz spielt für die Reaktionsdynamik eine wesentliche Rolle. In 
anderen Arbeitsgruppen sind verschiedene spektroskopische Methoden benutzt worden, um die Dynamik von ILs zu untersuchen. ${ }^{11-25}$ Hervorzuheben sind Untersuchungen der Wechselwirkung von molekularen Sonden mit ILs, wie z. B. durch ESR-Spektroskopie ${ }^{26127}$ und solvatochrome Verschiebung. ${ }^{28129}$ Die Lösungsdynamik von molekularen Sonden in ILs wird auch mittels verschiedener zeitaufgelöster spektroskopischer Methoden vom Subpikosekunden- bis Nanosekunden-Bereich untersucht. ${ }^{30-32}$ Überraschenderweise gibt es noch nicht so viele Messungen zur Charakterisierung der Wechselwirkung von Sonden mit ILs auf diesen ultrakurzen Zeitskalen, obwohl die mikroskopische Umgebung von Molekülen in Ils sehr wichtig für deren Dynamik und chemische Aktivität ist.

In der vorliegenden Arbeit werden Ergebnisse der Messungen ultraschneller photochemischer Prozesse von Farbstoffen in ILs diskutiert. Diese Messungen beruhen darauf, dass die Moleküle durch Absorption eines Photons angeregt werden und unter Emission von Strahlung zurück in den Grundzustand übergehen. Darüber hinaus sind viele verschiedene strahlungslose Wege für die Desaktivierung möglich, z. B. innere Konversion, intersystem crossing, intramolekularer Ladungstransfer und Konformationsänderungen. Die Charakteristik der Desaktivierungsprozesse, welche durch Wechselwirkungen des angeregten Moleküls mit seiner engsten Umgebung beeinflusst werden, kann Informationen über die Lösungsmittelumgebung liefern. So ist in früherer Arbeit die Lebensdauer der inneren Konversion von elektronisch angeregter 12 '-Apo- $\beta$-carotin-12'-säure (12'CA) in verschiedenen ILs untersucht worden, um Rückschlüsse auf die Mikroumgebung zu ziehen. $\frac{33}{3}$

Dieses Molekül zeigt eine ausgeprägte Änderung der Lebensdauer des ersten angeregten Zustands im Bereich von unpolaren (ca. 230 ps in Hexan) bis stark polaren (ca. 40 ps in Methanol ) organischen Lösungsmitteln. Diese starke Änderung ermöglicht auch die Untersuchung der spezifischen intermolekularen Wechselwirkung zwischen Sonde und der ionischen Flüssigkeit. Obwohl sich die ionischen Flüssigkeiten in ihrer chemischen Struktur und Ionenzusammensetzung beträchtlich unterscheiden, befinden sich alle Lebensdauern $\tau_{1}$ des ersten angeregten Zustands von 12'CA in einem eng definierten Bereich.

Die Ergebnisse deuten darauf hin, dass die Stabilisierung des angeregten Zustands von 12 'CA in allen ionischen Flüssigkeiten durch ähnliche Wechselwirkungen hervorgerufen wird. Wahrscheinlich dominiert hier eine elektrostatische Wechselwirkung zwischen dem Carbonylsauerstoff und der positiven Ladung des Kations der ionischen Flüssigkeit. Die 
Änderung der Lösungsmittelstruktur oder der chemischen Identität von Kationen und Anionen scheinen demgegenüber nur einen kleinen Beitrag zur Stabilisierung oder Destabilisierung des angeregten Zustands zu leisten. Ein solcher Beitrag wird deutlich, betrachtet man die imidazoliumbasierten ILs in Abbildung 1.1 (rote Strukturformel). Die Änderung von $\mathrm{R}_{2}=\mathrm{H}$ auf einen Methylrest bewirkt eine Zunahme der Lebensdauer $\tau_{1}$. Der angeregte Zustand kann nicht mehr so gut stabilisiert werden, da die Coulomb-Wechselwirkung mit dem Carbonylsauerstoff der Sonde schwächer ist.

Eine genauere Charakterisierung der Solvatation erfolgt in dieser Arbeit neben Messungen der transienten Absorption im nahen IR-Bereich, durch die erstmalige Anwendung der Breitband-Pump-Superkontinuum-Probe (PSCP) Spektroskopie auf molekulare Sonden in ionischen Flüssigkeiten. Dies ermöglicht eine Interpretation des Einflusses der ionischen Flüssigkeiten auf die Dynamik angeregter Zustände, die bisher noch nicht möglich war. Es sind zwei Klassen von Farbstoffen untersucht worden, zum einen die Carotinoide 12'Apo- $\beta$-carotin-12'-säure und 12'-Apo- $\beta$-carotin-12'-al, zum anderen indolinbasierte Farbstoffe, die in Grätzelzellen Anwendung finden. ${ }^{34}$ Grätzelzellen sind Farbstoffsolarzellen (Dye-Sensitized-Solar-Cells, DSSCs) die eine interessante Alternative zu den Siliziumsolarzellen darstellen. Als geeignete Elektrolyte haben sich ILs erwiesen. ${ }^{35}$ Ein großer Vorteil dieser Solarzellen sind die geringeren Produktionskosten. In puncto Effizienz können die DSSCs noch nicht mit den Siliziumsolarzellen konkurrieren. Grundlegende Forschung, wie die Untersuchung von Wechselwirkungen zwischen den eingesetzten Farbstoffen und ILs, ist notwendig, um die Prozesse in den DSSCs verstehen und letztendlich zur Verbesserung dieser Solarzellen beitragen zu können.

Zur Einordnung dieser Arbeit in die aktuelle Forschung auf dem Gebiet der ionischen Flüssigkeiten folgt zunächst ein kurzer Überblick über bisherige spektroskopische Methoden zur Untersuchung der Polarität von ILs mit Hilfe von molekularen Sonden. In Kapitel 3 werden die benutzten experimentellen Techniken erläutert. Anschließend werden in Kapitel 4 die Ergebnisse der ultraschnellen Dynamik von Carotinoiden in ILs präsentiert und diskutiert. Es folgen die Untersuchungen von binären Mischungen ionischer Flüssigkeiten mit Acetonitril. Kapitel 6 widmet sich den Farbstoffen für Solarzellen und deren Wechselwirkung mit ILs. 


\section{Kapitel 2}

\section{Mikroskopische Polarität von ionischen Flüssigkeiten: Spektroskopische Methoden}

Eine für diese Arbeit zentrale Eigenschaft der ionischen Flüssigkeiten (ILs) ist ihre Polarität, welche eine nicht einfach zu definierende Größe darstellt. Da die Polarität des Lösungsmittels einen Einfluss auf chemische Reaktionen haben kann, ist es möglich, durch die richtige Wahl des Lösungsmittels die Reaktion zu steuern. Eine Möglichkeit zur Bestimmung der Polarität von reinen ILs sind Messungen der Dielektrizitätskonstanten $\epsilon$. Dafür wird die Mikrowellenspektroskopie im Bereich von $100 \mathrm{MHz}-20 \mathrm{GHz}$ für viele ILs verwendet. Die meisten ILs haben $\epsilon$-Werte bei Zimmertemperatur im Bereich von 9-15 ${ }^{1}$, womit sie aus der Sicht der Mikrowellenspektroskopie als mäßig polar eingestuft werden. Die Dielektrizi-

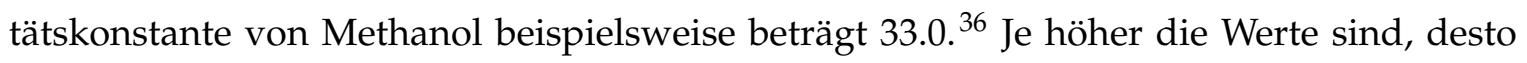
polarer ist das Lösungsmittel. Eine andere Sichtweise erhält man durch spektroskopische Methoden zur Untersuchung der mikroskopischen Umgebung von molekularen Sonden in ILs, die in diesem Kapitel vorgestellt werden.

Die wohl bekannteste Polaritätsskala ist die $E_{T}(30)-S k a l a$, welche durch die solvatochrome Verschiebung des Reichardt-Farbstoffs Betain-30 aufgestellt wird. ${ }^{29}$ An diese Methode der UV/Vis-Spektroskopie von solvatochromen Farbstoffen reihen sich noch weitere Polaritätsskalen, auf die in diesem Kapitel eingegangen wird. Im Kontrast zur Mikrowellenspektroskopie wird hierbei die „lokale Polarität“ der ILs vergleichbar mit kurzkettigen Alkoholen eingestuft. Es ist allerdings zu bemerken, dass die Ergebnisse manchmal widersprüchlich sind. Dies liegt wohl daran, dass die Korrelation zwischen der solvatochromen Verschiebung und der Lösungsmittelpolarität nicht so leicht zu definieren ist, da 
sowohl spezifische Wechselwirkungen, wie die Wasserstoffbrückenbindungen, als auch nicht-spezifische Wechselwirkungen, wie Ion-Dipol, Dipol-Dipol und Dipol-induzierter Dipol, eine Rolle spielen. Die Korrelation hängt in den verschiedenen Experimenten von der Natur des untersuchten Farbstoffes ab. Dies liegt in den verschiedenen Arten der Wechselwirkung zwischen Sonde und Lösungsmittel begründet, die je nach Art der Sonde unterschiedliche Bedeutung haben können. Eine Korrelation zwischen verschiedenen Wechselwirkungsparametern wird durch die Polaritätsskala nach KAMLET-ABBOUD-TAFT zugänglich. Diesen Polaritätsskalen von solvatochromen Farbstoffen widmet sich das Kapitel 2.1 .

Eine weitere Methode zur Untersuchung der Polarität von Ils ist die ESR-Spektroskopie von Radikalen. Zwischen den Radikalen (Spinprobes) und den ILs gibt es eine starke Wechselwirkung, die von der Natur der ionischen Flüssigkeit abhängt. Als Parameter dieser Messungen erhält man die Rotationskorrelationszeit $\tau$ und die Hyperfeinkopplungskonstante $\mathrm{A}_{i s o}\left({ }^{14} N\right)$, welche Informationen über die mikroskopische Polarität geben. ${ }^{37}$ Diese Methode wird in Kapitel 2.2 vorgestellt.

Die in dieser Arbeit untersuchte ultraschnelle Relaxation angeregter Zustände verschiedener Farbstoffe dient ebenfalls der Aufklärung der mikroskopischen Umgebung in ILs. Die Grundlage dieser Messungen wird in Kapitel 2.3 beschrieben.

\subsection{UV/Vis- und Fluoreszenz-Spektroskopie solvatochromer}

\section{Farbstoffe}

Wie oben erwähnt ist die Polarität von Lösungsmitteln nicht leicht zu beschreiben. Die Definition der IUPAC lautet: „Die Polarität ist die Summe aller möglichen, nicht-spezifischen und spezifischen, intermolekularen Wechselwirkungen zwischen gelösten Stoffen und dem Lösungsmittel, ohne die Wechselwirkungen die zu chemischer Veränderung des gelösten Stoffes führen würden.“ ${ }^{38}$ Die Untersuchung von solvatochromen Farbstoffen ist sehr einfach, da lediglich stationäre Spektroskopiemethoden angewandt werden müssen. Die Interpretation der Ergebnisse im Hinblick auf die Polarität der ionischen Flüssigkeiten ist allerdings nicht ganz so einfach. Im Folgenden werden zwei Methoden zur Aufstellung einer Polaritätsskala beschrieben. 


\subsubsection{Reichardts Farbstoff}

Lösungsmittel können einen starken Einfluss auf die Lage von Absorptionsbanden haben. Die Pyridinium- $N$-phenolat-Betainfarbstoffe eignen sich besonders als Indikator für die Lösungsmittelpolarität, da sie einen starken hypsochromen Shift mit steigender Polarität des Lösungsmittels zeigen. Bei der $E_{T}(30)$-Skala wird der Batain-Farbstoff Nr. 30 benutzt (2,6-Diphenyl-4-(2,4,6-triphenylpyridinium-1-yl)phenolat; s. Abb. 2.1), welcher auch als Reichardts Farbstoff bezeichnet wird. Dieser Farbstoff hat ein großes Dipolmoment $\left(\mu_{G}\right.$ $\approx 15 \mathrm{D}$ ), wodurch verschiedene Wechselwirkungen in den ILs registriert werden können. Des Weiteren beinhaltet er ein großes $\pi$-Elektronen System mit insgesamt $44 \pi$-Elektronen, was die Erfassung von Dispersionswechselwirkungen möglich macht. Eine Besonderheit sind die freien Elektronenpaare des Phenolatsauerstoffs, welche mit einem Wasserstoffbrückendonor interagieren können. 29

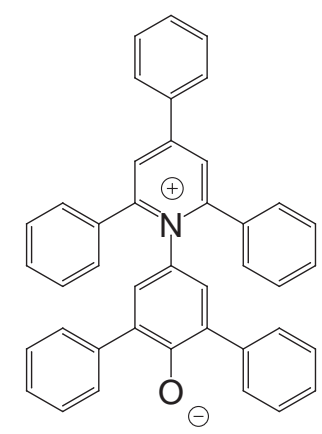

Abbildung 2.1: Reichardts Farbstoff

Die Dipolmomente des Grundzustands und des ersten angeregten Zustands differieren um 9 D, so dass mit steigender Polarität des Lösungsmittels der Grundzustand stärker stabilisiert wird. Von Diphenylether bis Wasser ergibt sich so eine hypsochrome Verschiebung von $\Delta \lambda=357 \mathrm{~nm}$ oder respektive $\Delta E_{T}=28 \mathrm{kcal} \cdot \mathrm{mol}^{-1}$. Die Polaritätsskala dieses Farbstoffes wird als $E_{T}(30)$-Skala bezeichnet. Definitionsgemäß handelt es sich hierbei um die molare Anregungsenergie in $\mathrm{kcal} \cdot \mathrm{mol}^{-1}$ des Betainfarbstoffs Nr. 30, welche bei Zimmertemperatur $\left(25^{\circ} \mathrm{C}\right)$ und 1 bar gemessen worden ist. Nach Gleichung 2.1 berechnen sich die Werte wie folgt:

$$
E_{T}(30) / k c a l \cdot \mathrm{mol}^{-1}=28591 /\left(\lambda_{\max } / \mathrm{nm}\right)
$$


Hierbei ist $\lambda_{\max }$ die Wellenlänge des Absorptionsmaximums vom intramolekularen Charge-Transfer Übergang $\pi-\pi^{*}$. Große $E_{T}(30)$-Werte sind ein Zeichen für eine hohe Lösungsmittelpolarität. Die Skala reicht von $63.1 \mathrm{kcal} \cdot \mathrm{mol}^{-1}$ in Wasser bis $30.7 \mathrm{kcal} \cdot \mathrm{mol}^{-1}$ für TMS. Zusätzlich wird eine dimensionslose Skala eingeführt. Diese wird als $E_{T}^{N}$-Skala bezeichnet. Als Referenz dient Wasser $\left(E_{T}^{N}=1.0\right)$ und TMS $\left(E_{T}^{N}=0.0\right)$. Ausgehend von den $E_{T}(30)$-Werten lässt sich eine $E_{T}^{N}$-Skala für ionische Flüssigkeiten berechnen. ${ }^{39}$

$$
E_{T}^{N}=\left[E_{T}(I L)-E_{T}(T M S)\right] /\left[E_{T}\left(H_{2} O\right)-E_{T}(T M S)\right]=\left[E_{T}(I L)-30.7\right] / 32.4
$$

Auf dieser Skala liegen die für diese Arbeit wichtigen imidazoliumbasierten Ils zwischen Cyclohexanol (0.5) und n-Propanol (0.6), also im mittelpolaren Bereich. Imidazoliumbasierte ILs kann man in zwei Klassen aufteilen, zum einen die 1-Methyl-3-alkyl-substituierten und zum anderen die mit zusätzlicher Methylgruppe an C-2-Position. Dem Wasserstoffatom an C-2 wird häufig die Fähigkeit zugeschrieben, Wasserstoffbrücken zu bilden. ILs mit aliphatischem Rest am C-2-Atom sind weniger polar, da sie keine Wasserstoffbrückenbindung eingehen können. Dies wird sehr gut mit der $E_{T}^{N}$-Skala wiedergegeben.

\subsubsection{Kamlet-Abboud-Taft-Parameter}

Nach KAMLET, ABBOUD und TAFT (KAT) werden Lösungsmittel durch ihre Polarisierbarkeit $\left(\pi^{*}\right)$ und die Wasserstoffbrückenbindungs-Donor- $(\alpha)$ und -Akzeptor-Fähigkeit $(\beta)$ beschrieben. ${ }^{40|41|}$ Diese drei Parameter können UV/Vis-spektroskopisch durch eine geeignete solvatochrome Probe ermittelt werden. In Gleichung 2.3 wird der Zusammenhang zwischen dem Absorptionsmaximum und den Polaritätsparametern deutlich. Diese Abhängigkeit wird auch als Linear Solvation Energy Relationship (LSER) bezeichnet.

$$
\nu_{\max }=\nu_{\max , 0}+a \alpha+b \beta+s \pi^{*}
$$

Die $\pi^{*}$-Werte können z. B. aus Messungen von 4-Nitroanisol oder N,N-Dimethyl-4-nitroanilin erhalten werden. ${ }^{42}$ Weitere solvatochrome Proben sind von SPANGE et. al bekannt. ${ }^{28}$ Die beiden Parameter zur Charakterisierung der Wasserstoffbrückenbindungseigenschaften $\alpha$ und $\beta$ erhält man durch Paare von solvatochromen Proben aus beispielsweise 4-Nitroanisol und dem Betain-Farbstoff Nr. $30 .{ }^{29}$ Es ergeben sich folgende Grenzen der drei Para- 
meter:
i) $\alpha=0.00$ (Cyclohexan) bis 1.96 (Hexafluoro-2-propanol)
ii) $\beta=0.00$ (Cyclohexan) bis 1.00 (Hexamethylphosphorsäuretriamid (HMPT))
iii) $\pi^{*}=0.00$ (Cyclohexan) bis 1.00 (Dimethylsulfoxid)

Um die Werte nach Gleichung 2.3 zu bestimmen, sollte eine Vielzahl von Lösungsmitteln untersucht werden, die sich in ihren KAT-Werten unterscheiden. Die Regressionskoeffizienten $a, b$ und $s$ sind hierbei unabhängig vom Lösungsmittel. Für viele ionische Flüssigkeiten sind die KAT-Parameter bereits bekannt. ${ }^{43}$ Die Werte für $\left[\mathrm{C}_{6} \mathrm{mim}\right]^{+}\left[\mathrm{Tf}_{2} \mathrm{~N}\right]^{-}$betragen $\alpha=0.69, \beta=0.25$ und $\pi^{*}=0.97$. Unter den organischen Lösungsmitteln ist kein Vertreter mit ähnlichen Eigenschaften zu finden. Der $\pi^{*}$-Wert ist etwas niedriger als der von Wasser und viel höher als der von kurzkettigen Alkoholen wie Methanol und Ethanol. Dahingegen ist der $\alpha$-Wert vergleichbar mit kurzkettigen Alkoholen und der $\beta$-Wert wieder wesentlich kleiner. Aufgrund der geringen Nukleophilie würde man diese ionische Flüssigkeit als nichtpolar einstufen, allerdings besitzt sie eine Polarisierbarkeit von polaren Lösungsmitteln. Eine Übersicht der Kamlet-Taft-Parameter für die in dieser Arbeit relevanten Lösungsmittel ist in Tabelle 2.1. gegeben.

Tabelle 2.1: Kamlet-Taft-Parameter

\begin{tabular}{lcccr}
\hline Lösungsmittel & $\alpha$ & $\beta$ & $\pi^{*}$ & Referenz \\
\hline$\left[\mathrm{C}_{6} \text { mim }\right]^{+}\left[\mathrm{Tf}_{2} \mathrm{~N}\right]^{-}$ & 0.69 & 0.25 & 0.97 & {$[40$} \\
{$\left[\mathrm{C}_{2} \mathrm{mim}\right]^{+}\left[\mathrm{EtSO}_{4}\right]^{-}$} & & & & \\
{$\left[\mathrm{C}_{4} \mathrm{mim}\right]^{+}\left[\mathrm{Tf}_{2} \mathrm{~N}\right]^{-}$} & 0.61 & 0.24 & 0.98 & 49 \\
{$\left[\mathrm{C}_{4} \mathrm{mmim}\right]^{+}\left[\mathrm{Tf} \mathrm{N}_{2}\right]^{-}$} & 0.38 & 0.24 & 1.01 & \\
{$\left[\mathrm{C}_{2} \mathrm{mim}\right]^{+}\left[\mathrm{N}(\mathrm{CN})_{2}\right]^{-}$} & & & & \\
{$\left[\mathrm{C}_{4} \mathrm{mim}\right]^{+}\left[\mathrm{B}(\mathrm{CN})_{4}\right]^{-}$} & & & & \\
Methanol & 0.93 & 0.62 & 0.60 & 40 \\
Ethanol & 0.83 & 0.77 & 0.54 & {$[40$} \\
Acetonitril & 0.19 & 0.31 & 0.75 & 40 \\
Tetrahydofuran & & 0.55 & 0.58 & {$[50$} \\
\hline
\end{tabular}




\subsection{ESR-Spektroskopie von Spinprobes in ionischen}

\section{Flüssigkeiten}

Radikale tauchen als reaktive Spezies in vielen chemischen Reaktionen auf. Das Mittel der Wahl zur Untersuchung von Radikalen in Lösungsmitteln ist die ESR Spektroskopie. Diese Methode ermöglicht Aussagen über die Mikropolarität von ionischen Flüssigkeiten (ILs). Hierbei wird die Wechselwirkung von Radikalen (Spinprobes) in dieser Art von Lösungsmittel betrachtet. Durch Veränderungen im ESR-Spektrum in Abhängigkeit von der Struktur der ionischen Flüssigkeit lassen sich Rückschlüsse auf deren mikroskopisches Verhalten ziehen. So sind bereits Piperidin-1-yloxyl-Derivate (siehe Abbildung 2.2) eingesetzt worden, um ILs zu untersuchen. $26 / 3751-55$

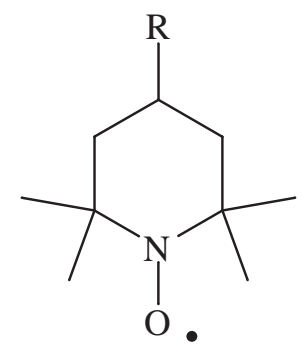

Abbildung 2.2: Struktur von Piperidin-1-yloxyl-Derivaten

Neben der Rotationskorrelationszeit $\tau$, die einen charakteristischen Parameter aus den ESR-Spektren darstellt, ist die isotropische Hyperfeinkopplungskonstante $\mathrm{A}_{\text {iso }}\left({ }^{14} \mathrm{~N}\right)$ wichtig zur Beschreibung der Wechselwirkungen in ionischen Flüssigkeiten. Die Diffusion des Radikals wird durch $\tau$ beschrieben. Hierbei lässt sich eine Abhängigkeit von der Viskosität der ionischen Flüssigkeit feststellen. ${ }^{56}$ Hervorgerufen durch elektrostatische Wechselwirkungen und Wasserstoffbrückenbindungen der Spinprobes mit dem Medium ändert sich die Verteilung der Spindichte, was im ESR-Spektrum detektiert werden kann. Eine maßgebliche Größe für die Spindichteverteilung ist $\mathrm{A}_{i s o}\left({ }^{14} \mathrm{~N}\right)$. Damit stellt dieser Parameter einen Indikator für die Wechselwirkungen mit den ionischen Flüssigkeiten dar. Natürlich ist zu beachten, dass die Art der Wechselwirkung auch immer von der Natur des Radikals abhängig ist. Trägt z. B. ein Piperidin-1-yloxyl-Derivat einen ionischen Substituenten $(R=$ $\mathrm{SO}_{4}{ }^{-}+\mathrm{K}^{+}$etc., s. Abb. 2.2, gibt es eine starke Wechselwirkung mit den jeweiligen Ionen der ionischen Flüssigkeit. 
Bei der Betrachtung von 1-Alkyl-2-methylimidazolium-bis(trifluoromethylsulfonyl)imid mit unterschiedlicher Kettenlänge konnte im Fall von geladenen Spinprobes eine Abhängigkeit der Hyperfeinkopplungskonstante $\mathrm{A}_{i s o}\left({ }^{14} \mathrm{~N}\right)$ von der Kettenlänge festgestellt werden. Obwohl die Polarität dieser imidazoliumbasierten ILs mit steigender Kettenlänge sinken sollte, zeigen die ESR-spektroskopischen Messungen ein anderes Bild. In weniger polaren Lösungsmitteln ist für gewöhnlich $\mathrm{A}_{i s o}\left({ }^{14} \mathrm{~N}\right)$ kleiner als in polaren. In den ILs steigt $\mathrm{A}_{\text {iso }}\left({ }^{14} \mathrm{~N}\right)$ allerdings mit wachsender Kettenlänge. Das würde bedeuten, dass die ILs mit großer Alkylkettenlänge polarer sind als die mit kleinerer Kette. Als mögliche Erklärung dafür wird die Tendenz zur Bildung von polaren und nichtpolaren Regionen in der ILs mit steigender Kettenlänge geboten. Die Vorstellung ist, dass sich dadurch anionische Spinprobes bevorzugt in den polaren Regionen aufhalten und es zu einer stärkeren Wechselwirkung mit dem Imidazoliumkation kommt. Im Gegensatz zu diesen Beobachtungen zeigen die kationischen und nicht geladenen Spinprobes keine Abhängigkeit der Hyperfeinkopplungskonstante. Bei diesen Spinprobes gibt es keine Wechselwirkung mit dem Imidazoliumkation. $\frac{56}{56}$

\subsection{Ultraschnelle Laserspektroskopie}

Die beiden vorangegangenen Kapitel widmen sich verschiedener Methoden zur Aufklärung der Polarität von ionischen Flüssigkeiten (ILs). Es ist gezeigt worden, wie molekulare Sonden verwendet werden können, um etwas über die mikroskopische Wechselwirkung in den ILs zu lernen. Dieses Kapitel liefert eine weitergehende Betrachtung dieser Wechselwirkungen. Es wird gezeigt, wie laserspektroskopische Methoden angewandt werden können, um etwas über die Solvatationsdynamik in den ILs zu erfahren. Bei diesen Methoden kommen ebenfalls molekulare Sonden zum Einsatz. Damit reihen sich die hier besprochenen und für diese Arbeit relevanten Methoden in den vorhergehend präsentierten Themenkomplex ein, liefern allerdings weitergehende Einblicke in die Welt der Mikropolarität von ionischen Flüssigkeiten.

Alle in diesem Kapitel besprochenen Methoden folgen dem gleichen Prinzip. Es ist die zeitabhängige Antwort von Molekülen auf eine Störung. Diese Störung wird durch einen Laserpuls verursacht, indem das Molekül angeregt wird. Den zeitlichen Verlauf der Relaxa- 
tionsdynamik, sprich die Umverteilung der Energie, die in dem Molekül deponiert worden ist, kann beispielsweise durch einen weiteren Laserpuls beobachtet werden. Die Prozesse, die hierbei in Erscheinung treten, laufen auf ultrakurzen Zeitskalen ab und sind abhängig von der Wahl des Lösungsmittels. Diese Art zur Untersuchung der Solvatationsdynamik und der Wechselwirkung der Sonde mit der Umgebung ist Gegenstand der vorliegenden Arbeit.

Die meisten ultraschnellen Messungen von Sonden in ionischen Flüssigkeiten beruhen auf der Time-Correlated Single-Photon-Counting (TCSPC)-Technik (zur Beschreibung dieser Technik sei auf die nachfolgenden Referenzen verwiesen), wohingegen in dieser Arbeit die transiente Absorptionsspektroskopie benutzt wird. Es gibt bereits eine große Menge an Daten zur Solvatationsdynamik in ionischen Flüssigkeiten, doch ergeben sich durch diese Arbeit Einblicke, die bisher noch nicht möglich waren (siehe Kapitel 4.5, 5.4 und 6.5). Angefangen hat das Interesse an diesen ultraschnellen Prozessen in ionischen Flüssigkeiten mit HupperT, der eine Klasse von Salzen mit Schmelzpunkten leicht oberhalb von $100{ }^{\circ} \mathrm{C}$ mittels TCSPC untersucht hat. ${ }^{[57-60]}$ Hierbei handelte es sich um Tetraalkylammoniumsalze, die nach aktueller Definition nicht mehr zu den ionischen Flüssigkeiten dazugezählt werden. Die Solvatationsdynamik in imidazoliumbasierten ILs ist erstmals von SAMANTA untersucht worden. ${ }^{61 / 62}$ Es folgen weitere Veröffentlichungen von SAMANTA ${ }^{63} \cdot 67$ und anderen Gruppen. ${ }^{24 \mid 68}+71$ Besonders hervorzuheben ist die Arbeit von MARONCELLI, $\stackrel{30 \mid 72+76}{-76}$ der unter anderem Messungen in einem Zeitintervall von $100 \mathrm{fs}-200$ ps durchgeführt hat. Ungefähr in diesem Regime liegen auch die Messungen der vorliegenden Arbeit.

Als fundamentales Ergebniss der TCSPC-Messungen ist festzuhalten, dass die Dynamik aus zwei Komponenten besteht. Eine schnelle Komponente, die der inertialen Eigenschaft der Ionen zuzuordnen ist und eine langsame Komponente, die mit der Viskosität der ionischen Flüssigkeit korreliert. Wie bereits aus Kapitel 2.1 und 2.2 ersichtlich sind die Ergebnisse aus diesen Messungen ebenfalls von der Beschaffenheit der molekularen Sonde abhängig. Die Solvatationsdynamik in ionischen Flüssigkeiten ist nicht leicht zu beschreiben, besonders die ultraschnelle Komponente wird noch nicht gut verstanden. SAMANTA schreibt: „Wegen der komplizierten Art des Problems und des Mangels an überzeugenden theoretischen Resultaten liegt es folglich auf der Hand, dass es durchaus einige Zeit dauern wird, bevor wir den Mechanismus der Solvatationsdynamik in ILs vollständig verstehen 
können.“ An diesem Punkt setzt die vorliegende Arbeit an, denn durch die Anwendung der ultraschnellen transienten Absorptionsspektroskopie ist es möglich, Aussagen über die allerersten Prozesse der Solvatation zu machen.

Es werden in dieser Arbeit zwei sich ergänzende Techniken der transienten Absorptionsspektroskopie angewandt (siehe Kapitel 3). Im nahen UV und sichtbaren Bereich wird die Breitband-Pump-Superkontinuum-Probe-Spektroskopie (PSCP) ${ }^{77}$ und im nahen IR ein transientes Zweifarben-Absorptionsexperiment (TA) benutzt. So können Informationen erhalten werden, die sich über einen weiten spektralen Bereich erstrecken. Das Grundprinzip der beiden Experimente ist identisch und soll im Folgenden erläutert werden.

In der transienten Absorptionsspektroskopie wird ein Teil der Moleküle durch einen Pumpstrahl in ein höher gelegendes elektronisches Niveau angeregt. Anschließend wird ein schwächerer Abfragepuls (Probepuls) mit einer Zeitverzögerung $\tau$ durch die Messzelle geleitet. Im Fall des PSCP-Experiment handelt es sich um ein Weißlicht-Probepuls und im TA-Experiment hat der Probepuls ein sehr enges Spektrum. Durch Änderung der Zeitverzögerung zwischen Pump- und Probepuls wird ein transientes Spektrum aufgenommen. Bei dem PSCP-Experiment kommt eine Abhängigkeit von der Zeit und der Wellenlänge bei nur einer Messung zustande. Im TA-Experiment müsste man bei jeder Messung eine neue Wellenlänge wählen. Durch Messungen mit der PSCP-Technik erhält man die transienten Spektren aus der Differenz des Absorptionsspektrums der angeregten Moleküle und dem der Moleküle im Grundzustand ( $\triangle \mathrm{OD}$ ). Es wird also die Änderung der optischen Dichte der Moleküle aufgenommen.

$$
\Delta O D=\log _{10}\left(\frac{I_{0}}{I}\right)_{p u m p}-\log _{10}\left(\frac{I_{0}}{I}\right)_{0} .
$$

Hierbei sind $\log _{10}\left(\frac{I_{0}}{I}\right)_{\text {pump }}$ und $\log _{10}\left(\frac{I_{0}}{I}\right)_{0}$ die negativen Extinktionen der Sonden mit und ohne Anregung durch einen Pumppuls. Die $\Delta$ OD-Spektren enthalten immer Beiträge verschiedener Prozesse. Einer dieser Beiträge ist der so genannte Ground-State Bleach (GSB). Da ein Teil der Moleküle angeregt worden ist, ist die Absorption aus dem Grundzustand geringer als vor der Anregung. Daher kommt es im Wellenlängenbereich der Grundzustandsabsorption zu einem negativen transienten Signal. Ein weiterer Beitrag könnte eine stimulierte Emission (SE) sein. In einem Zwei-Niveau-System sind die Einstein-Koeffizienten für die Absorption vom Grundniveau in das angeregte Niveau $\left(\mathrm{B}_{12}\right)$ und die stimulier- 
te Emission zurück in das Grundniveau $\left(B_{21}\right)$ gleich. Daher kann nach Anregung durch den Pumpstrahl eine stimulierte Emission hervorgerufen vom Probestrahl erfolgen. Das Spektrum der stimulierten Emission entspricht dem Fluoreszenzspektrum der molekularen Sonde multipliziert mit einem Faktor $\frac{1}{\nu^{2}}$, ist also im Vergleich zur Absorption stokesverschoben. Im transienten Spektrum erscheint sie ebenfalls als negatives Signal.

Aus angeregten Zuständen kann ebenfalls eine Absorption erfolgen, die so genannte Excited-State Absorption (ESA). Da hierbei ein Teil des Probepulses für den Übergang aus dem angeregten in ein noch höheres Niveau absorbiert wird, erscheint dieser Beitrag als positives Signal im transienten Spektrum. Als weiterer Beitrag zum transienten Spektrum könnte noch eine Absorption von einem möglicherweise gebildeten Produkt beobachtet werden. So entsteht z. B. bei den in dieser Arbeit gemessenen Carotinoiden durch eine Zwei-Photonen-Absorption ein langlebiges Radikalkation. Dieses Radikalkation absorbiert einen Teil des Probepulses im langwelligen Bereich des Spektrums und erscheint als positives Signal. Alle hier besprochenen Beiträge sind nochmals in Abbildung 2.3 verdeutlicht. Die technischen Details der transienten Absorptionsspektroskopie folgen in Kapitel 3 .

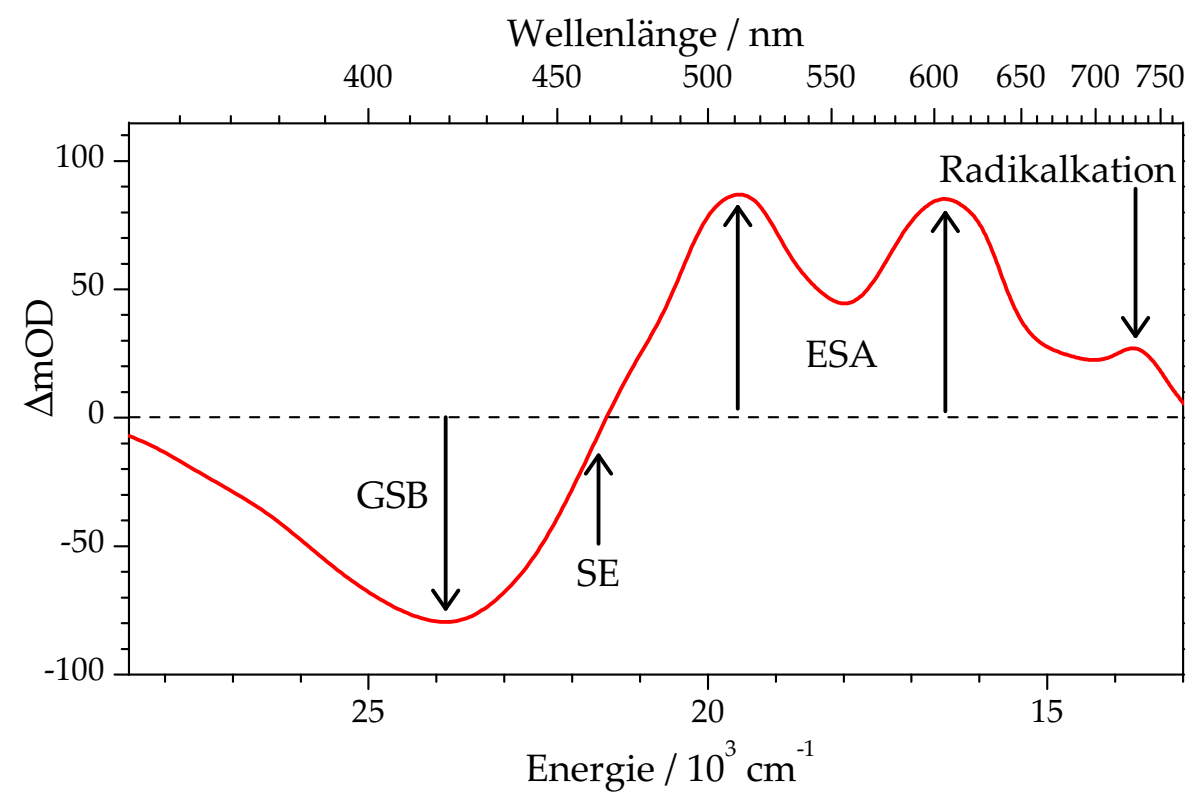

Abbildung 2.3: Beiträge zu einem $\triangle \mathrm{OD}$ Spektrum: Ground-State Bleach (GSB), stimulierte Emission (SE), Excited-State Absorption (ESA) und Absorption eines Radikalkations. 


\section{Kapitel 3}

\section{Experimentelle Technik}

Die Dynamik angeregter Zustände der betrachteten Farbstoffe wird durch zwei sich ergänzende Pump-Probe-spektroskopische Methoden untersucht. Zur Aufnahme transienter Spektren vom ultravioletten bis Nah-IR-Bereich (355 - 775nm) wurde ein Breitband-PumpSuperkontinuum-Probe-Spektroskopie (PSCP)-Aufbau benutzt. ${ }^{77}$ Noch weiter im Roten kann die Dynamik mit einem ultraschnellen transienten Absorptionsexperiment (TA) untersucht werden. Beide Experimente haben sich für entsprechende Untersuchungen von Farbstoffen in organischen Lösungsmitteln bewährt. ${ }^{78-81}$ Nach ersten Messungen von 12'Apo- $\beta$-carotin-12'-säure in ionischen Flüssigkeiten mittels transienter Absorptionsspektroskopie $\mathrm{e}^{33}$ konnten im Rahmen der vorliegenden Arbeit erstmals transiente Spektren verschiedener Farbstoffe mit der PSCP-Technik in dieser Art von Medium bestimmt werden. ${ }^{8283}$ Dies ermöglicht eine Interpretation des Einflusses der ionischen Flüssigkeiten auf die Dynamik angeregter Zustände, die bisher noch nicht möglich war.

Der Einführung in die Femtosekundenspektroskopie wird eine detaillierte Beschreibung der experimentellen Aufbauten folgen. Eine zentrale Untersuchung dieser Arbeit behandelt Mischungen von ionischen Flüssigkeiten und organischen Lösungsmitteln. Daher werden am Ende des Kapitels Grundlagen der Solvatochromie vorgestellt, welche unter anderem als Methode zur Messung dieser Mischungen gewählt wird. 


\subsection{Prinzip der Femtosekundenspektroskopie}

Dynamische Prozesse von Molekülen (z. B. Relaxationen angeregter Zustände) laufen auf ultrakurzen Zeitskalen ab. Um deren Entwicklung zu studieren, bedarf es zeitaufgelöster Spektroskopiemethoden. Werden Laser als Lichtquelle dieser Methoden benutzt, muss das Zeitintervall $\Delta \mathrm{t}$ der Pulse kleiner sein als die Dauer $\tau$ des zu untersuchenden Vorganges. Die Dynamik angeregter Zustände der in dieser Arbeit untersuchten Farbstoffe bewegt sich in einem Zeitintervall von mehreren hundert Femtosekunden bis zu mehreren hundert Pikosekunden. Zur Messung dieser Dynamik sind Femtosekundenpulse notwendig.

Ein Mittel um ultrakurze Prozesse zu messen, ist die Pump-Probe-Spektroskopie, welche eine höhere Zeitauflösung erreicht als Spektroskopiemethoden unter Benutzung herkömmlicher Detektoren. Bei dieser Methode werden die Moleküle durch einen Laserpuls (Pumppuls) angeregt und können durch einen weiteren Puls (Probepuls) abgefragt werden. Die zeitliche Entwicklung der angeregten Zustände kann durch die Verzögerung von Pump- und Probepuls erfasst werden. Allein die Dauer beider Pulse bestimmt die zeitliche Auflösung und nicht der Detektor.

Ein System, welches vielfältige Anwendungsmöglichkeiten besitzt, ist der Titan-Saphir (Ti:Saphir)-Laser. Hiermit lässt sich eine Zeitauflösung von wenigen Femtosekunden erreichen. ${ }^{84}$ Das Prinzip, welches der Erzeugung ultrakurzer Pulse im Ti:Saphir-Laser zugrunde liegt, ist die Kerr-Linsen-Modenkopplung. Aufgrund des nichtlinearen intensitätsabhängigen Brechungsindexes

$$
n(I)=n_{0}(\omega)+n_{2}(\omega) \cdot I
$$

wird der Laserstrahl im Kristall fokussiert. Hierbei ist $n_{0}$ der lineare Brechungsindex und $n_{2}$ der Kerr-Koeffizient. Hohe Intensitäten des Lasers werden stärker fokussiert als niedrige. Für einen gaußförmigen Puls nimmt der Brechungsindex zur Mitte des Strahls hin zu. Dies führt dazu, dass die Lage der Fokusebene während des Pulses variiert. Durch geeignete Ausblendung der niedrigen Intensitäten (cw-Moden) werden diese entfernt und nur der intensive modengekoppelte Puls wird verstärkt. $\frac{85}{6}$

In den folgenden Kapiteln werden die für diese Arbeit relevanten laserspektroskopischen Techniken erläutert. In beiden Experimenten werden Ti:Saphir-Laser unterschiedli- 
cher Bauweise benutzt. Die grundlegende Methode ist in beiden Fällen die Pump-ProbeSpektroskopie.

\subsection{Breitband Pump-Superkontinuum-Probe-Spektroskopie}

Die Breitband Pump-Superkontinuum-Probe-Spektroskopie (PSCP) ist eine wirkungsvolle Methode zur Untersuchung der Dynamik angeregter Zustände. Diese Methode ermöglicht es, mit einer einzelnen Messung eine große spektrale Breite (ca. $15000 \mathrm{~cm}^{-1}$ ) zu erfassen. Dies wird erreicht durch die Erzeugung eines Weißlichtkontinuums, welches als einzelner gechirpter Puls betrachtet werden kann. Daraus resultiert das Problem einer genauen Zeitkorrektur der transienten Spektren, das durch die Chirp-Korrektur (siehe Kapitel 3.2.4) des Signals eines reinen Lösungsmittels gelöst werden kann. $77 / 86$

Das verwendete Lasersystem ist ein Ti:Saphir-Oszillator-regenerativer Verstärker (Hurricane, Spectra-Physics, 780 nm, 920 Hz, 100 fs, 1 mJ/Puls), welches in Kapitel[3.2.1]näher beschrieben wird. Die Pump- und Probepulse werden in zwei Non-Collinearly Phase-Matched Optical Parametric Amplifiers (NOPAs) erzeugt. Die Pulse des Probe-NOPAs werden zur Erzeugung eines Weißlichtspektrums auf ein $1 \mathrm{~mm}$ dickes $\mathrm{CaF}_{2}$-Plättchen fokussiert. Eine genaue Beschreibung der Erzeugung von Pump- und Probepulsen erfolgt in Kapitel 3.2.2 und 3.2 .3 .

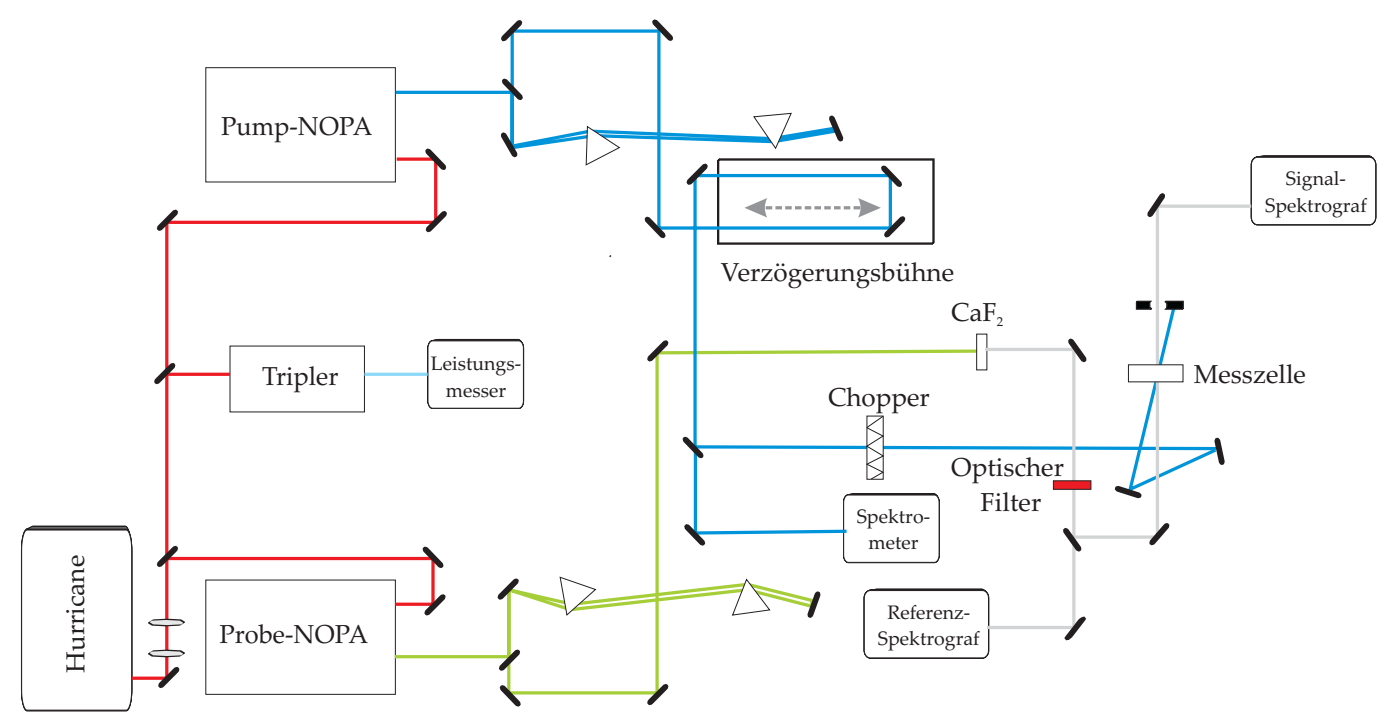

Abbildung 3.1: Schematischer Aufbau des PSCP Experiments. 
Der Strahlengang des PSCP-Experiments ist in Abbildung 3.1 illustriert. Zur täglichen Optimierung der Intensität und Zeitauflösung der Fundamentalen aus dem Hurricane wird der Puls nach Austreten aus dem Tripler auf maximale Energie eingestellt. Je kürzer die Pulse sind, desto besser funktioniert die Verdreifachung der Pulsfrequenz im Tripler und um so höher ist dementsprechend die Energie. Der Fundamentalpuls (780 nm) wird zum Pumpen von zwei NOPAs verwendet. Die NOPA-Pulse sind aufgrund von enthaltenen dispersiven Elementen verlängert. Daher werden sie jeweils durch ein Paar von Quarzprismen komprimiert. Der Pumppuls wird dann über eine Verzögerungsbühne geleitet und in eine Messzelle fokussiert. Der Strahl aus dem Probe-NOPA wird zur Erzeugung von Weißlicht-Pulsen in einem $1 \mathrm{~mm}$ dicken $\mathrm{CaF}_{2}$-Kristall benutzt. Nach spektraler Glättung in einer Farbstofflösung und Aufteilung in Referenz- und Signalstrahl werden die Probepulse ebenfalls in die Messzelle fokussiert.

Die Spektren von Referenz- und Signalstrahl werden in zwei separaten Spektrografen aufgelöst und durch Photodioden mit 512 Elementen detektiert. Die relative Polarisation von Pump- und Probe-Strahl ist bei allen Messungen im magischen Winkel. Jedes transiente Spektrum wird 1500 mal gemittelt. Die Zeitauflösung des Aufbaus beträgt 60 bis 80 fs. Eine genaue Beschreibung des PSCP-Aufbaus ist bereits an anderer Stelle veröffentlicht worden. 7786

\subsubsection{Das Hurricane-System}

Das Hurricane-Lasersystem der Firma Spectra-Physics erzeugt ultrakurze Pulse mit einer Dauer von ca. 100 fs. Dieses System kombiniert die vier typischen Komponenten eines Kurzpuls-Festkörperlasers in einem Gerät: Oszillator, Strecker, regenerativer Verstärker und Kompressor (siehe Abbildung 3.2). Es handelt sich um ein abgeschlossenes und vollständig temperierbares System, wobei der Oszillator und der Verstärker einen voneinander getrennten Kühlkreislauf aufweisen. 87

Ein Bauteil des Hurricanes ist der so genannte MaiTai (Spectra-Physics), welcher den Ti:Saphir Oszillator enthält. Als Pumplaser wird ein diodengepumpter Nd:YAG-Laser mit einer zentralen, in einem LBO-Kristall verdoppelten, Wellenlänge von $532 \mathrm{~nm}$ benutzt. Der Ti:Saphir-Oszillator wird mit einer konstanten Leistung von $5 \mathrm{~W}$ gepumpt. Innerhalb des Resonators befindet sich ein akustooptischer Modulator, der die Modenkopplung der Pul- 


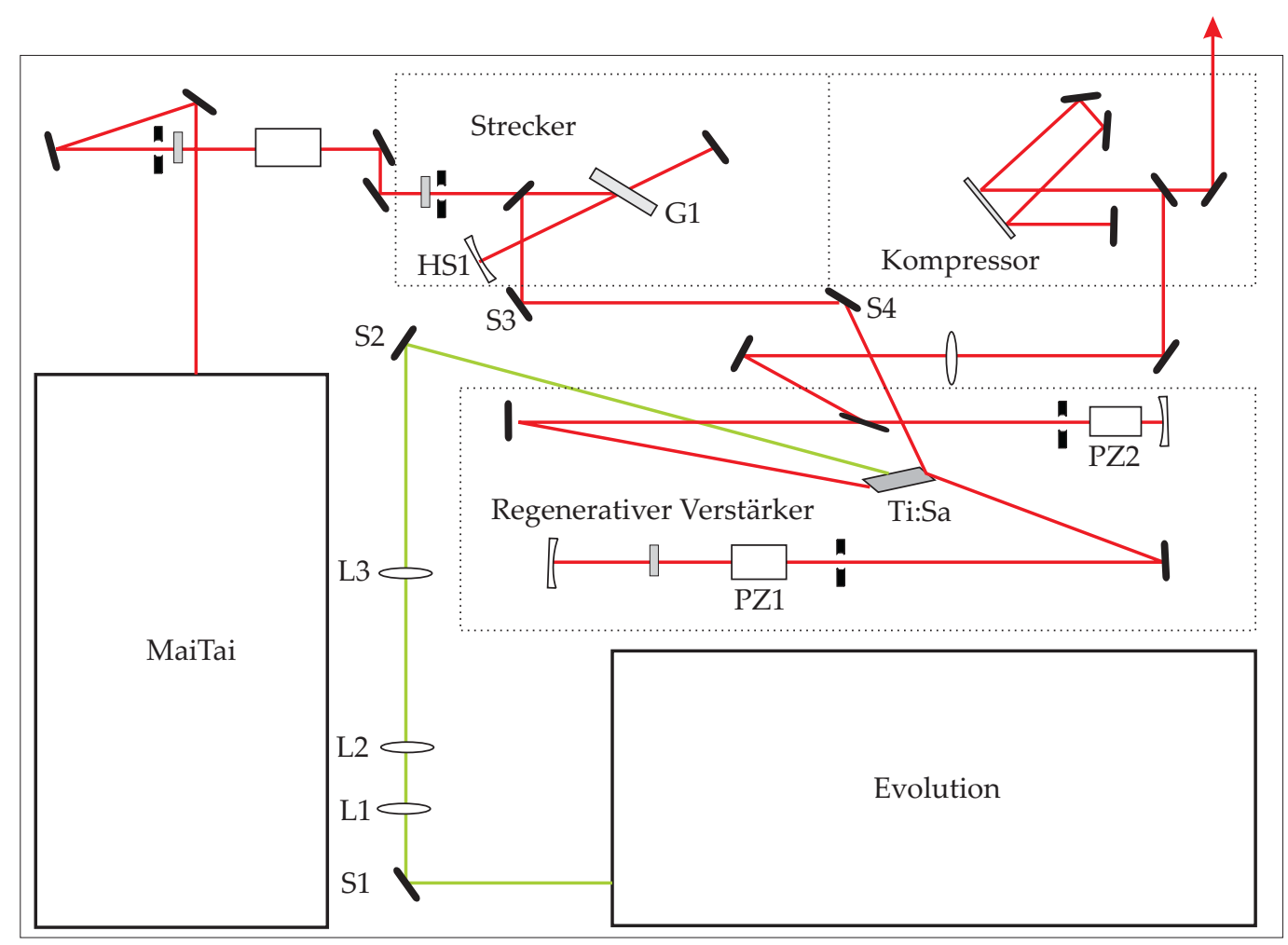

Abbildung 3.2: Aufbau des Hurricane-Systems.

se unterstützt. Die Emissionswellenlänge eines Ti:Saphir-Lasers ist im Fall des MaiTais von 780-820 nm durchstimmbar und wurde für das Experiment dieser Arbeit auf $780 \mathrm{~nm}$ festgelegt. Die in den optischen Elementen auftretende Gruppengeschwindigkeitsdispersion wird durch ein Prismenpaar innerhalb des Resonators ausgeglichen.

Ultrakurze Pulse weisen ein relativ breites Spektrum auf. Es ist möglich, diese mit Hilfe von Prismen oder Gittern zeitlich zu strecken, um ihre Spitzenintensitäten zu verringern. Der Hurricane-Strecker macht sich dies nutzbar, um eine Schädigung des Laserkristalls zu verhindern. Eine Anordnung von Gitter (G1) und Goldhohlspiegeln (HS1) (siehe Abbildung 3.2) ermöglicht eine Verzögerung der blauen spektralen Anteile gegenüber den roten Anteilen und somit eine Verlängerung der Pulse.

Die gestreckten Pulse werden anschließend im regenerativen Verstärker selektiert und maximal verstärkt. In einem weiteren Ti:Saphir-Kristall wird den Pulsen vom Evolution (Spectra-Physics) zusätzliche Energie zugeführt. Dies ist ein diodengepumpter, frequenzverdoppelter Neodym-Yttriumlithiumfluorid-Laser (Nd:YLF), der durch einen Q-Switch ca. 50 ns lange Pulse mit einer Wellenlänge von $527 \mathrm{~nm}$ und einer Repetitionsrate von 920 
$\mathrm{Hz}$ hat. Die vom Evolution emittierten Pulse werden in einem Teleskop (L1 und L2, siehe Abbildung 3.2 kollimiert, von L3 fokussiert und durch die Spiegel S1 und S2 in den regenerativen Verstärker eingekoppelt. Die Pulse aus dem MaiTai werden nach dem Strecker über die Spiegel S3 und S4 an dem Ti:Saphir im regenerativen Verstärker reflektiert. Bei deaktivierter Einkoppel-Pockelszelle (PZ1) kommt es nicht zur Verstärkung. Durch das Öffnen des Evolution-Q-Switches trifft ein Pumppuls auf den Ti:Saphir-Kristall, PZ1 wird aktiviert und der Verstärkungsprozess kann beginnen. Ist die maximale Verstärkung erreicht, wird die Auskoppel-Pockelszelle (PZ2) aktiviert und der Strahl kann schließlich zum Kompressor gelangen.

Im Kompressor werden die Pulse mittels Retroreflektoren und einem Gitter auf ca. 100 fs komprimiert. Einer dieser Reflektoren befindet sich auf einer elektronisch steuerbaren Translationsbühne und kann auf niedrigste Pulsdauern optimiert werden. Die Hurricane-Laserpulse haben eine Wellenlänge von $780 \mathrm{~nm}$ mit einer Energie von $1 \mathrm{~mJ}$ pro Puls und einer Repetitionsrate von $920 \mathrm{~Hz}$.

\subsubsection{Erzeugung der Anregungspulse}

Die Anregungspulse werden mit einem NOPA erzeugt. Dieser stellt einen optisch-parametrischen Verstärker zur Erzeugung von Femtosekundenpulsen dar. Die Verstärkung von Laserlicht erfolgt in einem optisch nichtlinearen Kristall (z. B. BBO). Hier wird ein so genannter Signalpuls $\left(\omega_{s}\right)$ mit einem Pumppuls $\left(\omega_{p}\right)$ nicht kollinear überlagert (siehe Abbildung 3.3. Der Pumppuls ist dabei sehr viel stärker als der Signalpuls. Durch die Nichtlinearität des Kristalls können eine Vielzahl neuer Frequenzen entstehen. Der Winkel des Kristalls muss so eingestellt sein, dass die Phasengeschwindigkeiten von Signal- und Pumppuls aufeinander abgestimmt sind, damit nur die Welle der Differenzfrequenz erzeugt wird. Diese Welle wird als Idler $\left(\omega_{i}\right)$ bezeichnet. 88

$$
\omega_{i}=\omega_{p}-\omega_{s}
$$

Der NOPA besitzt zwei Verstärkungsstufen. In der ersten Stufe wird ein Weißlicht als Signalpuls verwendet. Dieses Weißlicht wird durch die fundamentale Laserstrahlung des Hurricanes $(780 \mathrm{~nm})$ in einem Saphirkristall erzeugt. Bei der Erzeugung des Weißlichts entsteht ein Chirp, da sich im Medium nicht alle Wellenlängen mit der gleichen Geschwindig- 
keit ausbreiten, wodurch eine Verlängerung der Pulse zustande kommt. Daher erreichen nicht alle Anteile des Weißlichts den BBO-Kristall, in dem der parametrische Prozess stattfinden soll, zur gleichen Zeit. Durch zeitliche Verzögerung zwischen dem Pumppuls und dem Weißlicht-Signal kann somit ein bestimmter Wellenlängenbereich verstärkt werden. Als Pumppuls für die parametrische Verstärkung dient die in einem BBO frequenzverdoppelte Strahlung (390 nm). In der zweiten Stufe wird auf gleiche Weise der ausgewählte Wellenlängenbereich weiter verstärkt.

Als Herzstück der parametrischen Verstärkung sind die nichtlinearen Kristalle anzusehen. Die wichtigste Eigenschaft dieser Medien ist deren Polarisierbarkeit. Die Polarisation kann hierbei als Funktion des elektrischen Feldes beschrieben werden:

$$
P(E)=\epsilon_{0} \cdot \sum_{n} \chi^{n} E^{n}=\epsilon_{0} \chi^{1} E^{1}+\epsilon_{0} \chi^{2} E^{2}+\epsilon_{0} \chi^{3} E^{3} \cdots
$$

Hierbei entspricht $\chi$ der elektrischen Suszeptibilität und $\epsilon_{0}$ ist die elektrische Feldkonstante. Im sichtbaren Spektralbereich haben Festkörper elektrische Suszeptibilitäten in der Größenordnung von $\chi^{1} \approx 1, \chi^{2} \approx 10^{-10} \mathrm{~m} / \mathrm{V}$ und $\chi^{3} \approx 10^{-17} \mathrm{~m}^{2} / \mathrm{V}^{2} .89$ Die lineare Suszeptibilität ist also sehr viel größer. Die nicht linearen Terme können nur bei sehr starken Feldern, wie es bei einem Laser der Fall ist, beobachtet werden. Die parametrische Verstärkung ist ein Beispiel für einen Effekt zweiter Ordnung.

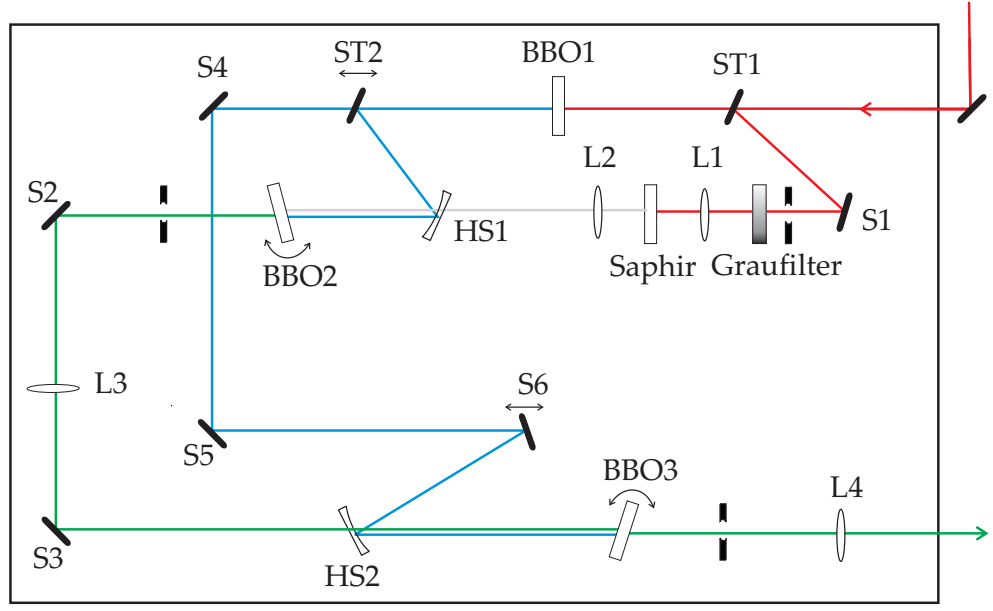

Abbildung 3.3: Optischer Aufbau des NOPA Systems. 


\subsubsection{Superkontinuumserzeugung}

Als Probepuls wird ein Weißlichtkontinuum verwendet, welches als einzelner gechirpter Puls angesehen werden kann. Es gibt verschiedene Techniken zur Erzeugung solcher Pulse. Breitbandige Weißlichtpulse lassen sich beispielsweise mit gasgefüllten Hohlfasern ${ }^{90}$ oder dünnen Quarzfasern ${ }^{91}$ erzeugen. Eine weitere Methode ist die Verwendung transparenter Festkörper. ${ }^{92}$ In dieser Arbeit wird das Superkontinuum in einem Calciumfluoridkristall $\left(\mathrm{CaF}_{2}\right)$ erzeugt. Dieser muss ständig in Bewegung gehalten werden, damit er durch die Fokussierung des Lasers nicht beschädigt wird. Für die Erzeugung des Weißlichts wird der Output eines weiteren NOPAs (ca. $560 \mathrm{~nm}$ ) verwendet. Das Kontinuum muss durch eine Farbstofflösung spektral gefiltert werden, um das Spektrum zu glätten. Ein Beispiel des Weißlichtspektrums ist in Abbildung $3.4 \mathrm{zu}$ finden.

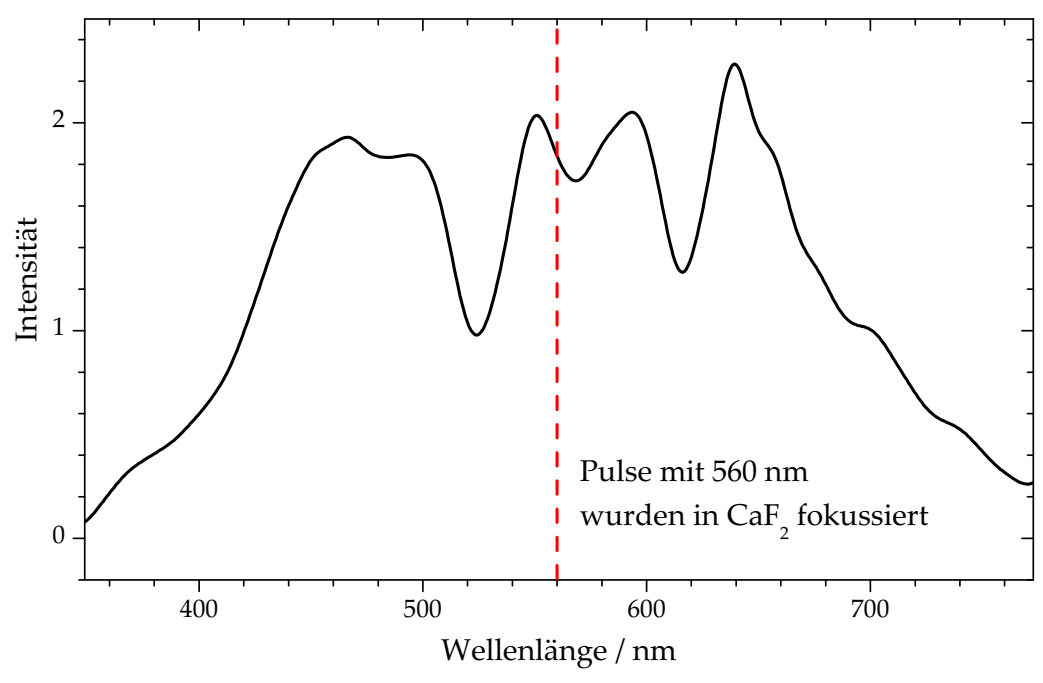

Abbildung 3.4: In $\mathrm{CaF}_{2}$ erzeugtes Weißlichtspektrum.

Die Effekte, die bei der Erzeugung des Superkontinuums eine Rolle spielen, sind die Selbstfokussierung und die Selbstphasenmodulation. Hierbei handelt es sich um nichtlineare Effekte. In einem nichtlinearen Medium wird durch den eintretenden Strahl über die Suszeptibilität dritter Ordnung (siehe Gleichung 3.3 die Brechzahl so verändert, dass die Intensität des Strahls zum Zentrum gebündelt wird (Voraussetzung: gaußförmiges Strahlenprofil). Bei diesem Effekt spricht man von einer Selbstfokussierung. Mit dieser Brechzahländerung $\Delta n$ tritt eine proportionale Änderung der Phase des Strahls $\Delta \Phi$ auf, die so genannte Selbstphasenmodulation. Physikalisch entspricht dem eine zeitliche Verschie- 
bung der $\Delta \nu(t)$ Frequenz des Strahls. An Vorder- und Rückflanke der Pulse ändert sich die Intensität besonders schnell, daher ist dort die Frequenzverschiebung am größten. Je kürzer ein Puls ist, desto steiler sind dessen Flanken, was zu einem breiteren Spektrum der austretenden Pulse führt. ${ }^{93}$ Die Femtosekundenpulse aus dem NOPA (ca. 60 fs) erzeugen ein Weißlichtspektrum mit etwa $420 \mathrm{~nm}$ Breite.

\subsubsection{Chirp-Korrektur}

Das Weißlicht ist aufgrund der Selbstphasenmodulation und der Gruppengeschwindigkeitsdispersion im $\mathrm{CaF}_{2}-$ Kristall (siehe Kapitel 3.2.3 gechirpt. Das bedeutet das unterschiedliche spektrale Bereiche des Pulses zu unterschiedlichen Zeiten in der Messzelle ankommen (siehe Abbildung 3.5. Daher müssen die transienten Spektren zeitlich korrigiert werden, die so genannte Chirp-Korrektur.

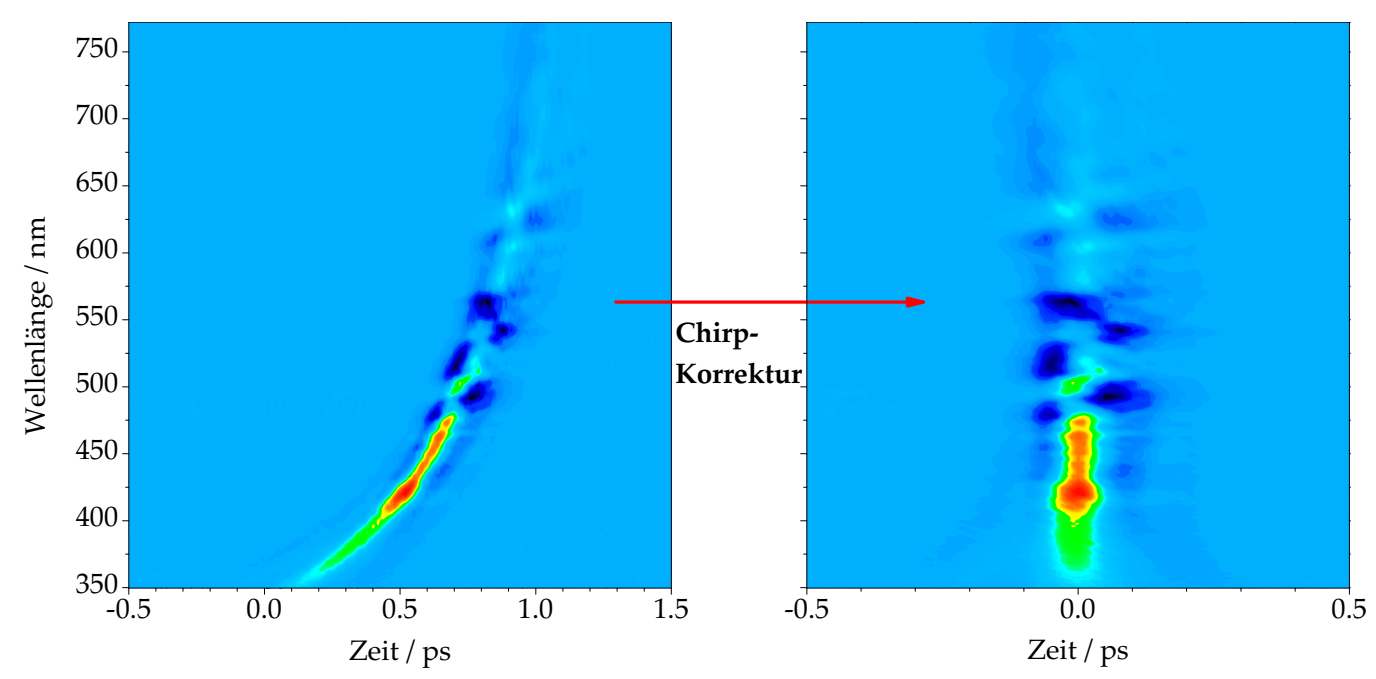

Abbildung 3.5: Nichtresonantes kohärentes Signal einer ionischen Flüssigkeit vor und nach der Chirp-Korrektur.

Die zeitliche Korrektur erfolgt anhand des nichtresonanten kohärenten Signals von reinen Lösungsmitteln. In Abbildung 3.5 ist ein solches charakteristisches Signal für ionische Flüssigkeiten exemplarisch dargestellt. Die Messung der reinen ionischen Flüssigkeit erfolgt vor jeder regulären Messung einer molekularen Sonde in ionischen Flüssigkeiten. Die zeitliche Verschiebung, die sich durch den Chirp ergibt, wird durch eine Funktion angepasst und kann anschließend korrigiert werden. Dann sind die Maxima der Intensitäten aller Wellenlängen auf den zeitlichen Nullpunkt geschoben, so wie es bei nicht gechirpten 
Pulsen der Fall wäre. Anhand der Chirp-Korrektur des Signals der reinen ionischen Flüssigkeiten werden alle nachfolgend gemessenen transienten Signale korrigiert. Schließlich ist es dann auch möglich das nichtresonante kohärente Signal des Lösungsmittels von den Signalen der molekularen Sonde abzuziehen, sodass man die reinen transienten Signale dieser Moleküle in den ionischen Flüssigkeiten bei kurzen Zeiten erhält.

\subsection{Ultraschnelles transientes Absorptionsexperiment}

Zur laserspektroskopischen Untersuchung der Farbstoffe in ionischen Flüssigkeiten im nahen IR wird das Tsunami-System der Firma Spectra-Physics verwendet. Eine genaue Beschreibung des Lasers erfolgt in Abschnitt 3.3.1. Es handelt sich hierbei um einen Ti:SaphirLaser, der durch Kerr-Linsen-Modenkopplung ultrakurze Pulse erzeugt. Eine Besonderheit dieses Experiments ist die Detektion der Signale. Hierbei kommt eine Lock-In-Technologie zum Einsatz, die in Abschnitt 3.3.3 näher erläutert wird.

In Abbildung 3.6 ist der optische Aufbau für das transiente Absoprtionsexperiment gezeigt. Abgesehen vom Strahlengang gibt es drei wichtige Bereiche, dies sind das LaserSystem (A), die Erzeugung der Pumppulse (B) und die Detektion der Signale (C).

Der Klappspiegel (S1) dient zum Umlenken des ausgekoppelten Laserstrahls auf den Leistungsmesser A, welcher zur Optimierung des Tsunamis benötigt wird. Durch einen Strahlteiler (ST) wird der Laserstrahl in zwei Strahlen geteilt. Der Strahlteiler sorgt durch seine Eigenschaft (Laseroptik, 10 \% Transmission und 90 \% Reflexion; Angabe bei $800 \mathrm{~nm}$ ) dafür, dass die beiden Strahlen unterschiedliche Energien haben. Durch einen weiteren Klappspiegel (S2), der auf einem Spektrometer justiert werden kann, ist es möglich, das Spektrum der Laserpulse zu beobachten und somit die gewünschte Wellenlänge einzustellen.

Das Teleskop (L1 und L2) kollimiert den transmittierten Teil, der für die Erzeugung der Pumppulse verwendet wird (siehe Abbildung 3.6). Im akusto-optischen Modulator (AOM, Isomet 1205C-2) befindet sich ein Bleimolybdat-Kristall, in dem ein Piezoelement ein periodisch optisches Gitter mit einer Frequenz von $2 \mathrm{MHz}$ erzeugt. Der AOM wird von einem Hochfrequenztreiber (Landwehr A103) angesteuert, welcher von einem Pulsgenerator (Hewlett Packard 8111A) getriggert und von einem 24V-Gleichspannungsnetzteil (Volt- 
craft) versorgt wird. Das frequenzmodulierte erste Beugungsmaximum wird durch einen LBO-Kristall (2 $\mathrm{mm}$ ) frequenzverdoppelt. Der nicht verdoppelte Anteil wird durch einen dichroitischen Spiegel abgetrennt. Der verdoppelte Anteil des Laserstrahls wird mit einer Sammellinse (L5, Suprasil I, $\mathrm{f}=75 \mathrm{~mm}$ ) fokussiert und als Pumpstrahl durch die Messzelle geleitet.

Eine computergesteuerte Verzögerungsbühne (Physik Instrumente (PI), M-531.GG) sorgt für die zeitliche Verzögerung zwischen Pump- und Probestrahl. Sie besitzt eine Weglänge von $30 \mathrm{~cm}$, womit eine Verzögerungszeit von bis zu 2 ns möglich ist. Der am Strahlteiler (ST) reflektierte Teil passiert einen Polarisator (P) und eine $\lambda$ / 2-Platte um den magischen Winkel zwischen Pump- und Probe-Strahl einstellen zu können. Der Probe-Strahl wird ebenfalls mit L5 fokussiert. Beide Foki werden mit Hilfe eines Pinholes (50 $\mu \mathrm{m}$ ) übereinander gelegt. Während des Experiments befindet sich die Messzelle kurz vor oder hinter dem Fokus. Eine zweite Sammellinse (L6, Suprasil I, $\mathrm{f}=50 \mathrm{~mm}$ ) ist in ca. $15 \mathrm{~cm}$ Entfernung angebracht, um den Probe-Strahl auf eine Avalanche-Photodiode zu fokussieren (APD, Hamamatsu S2381, aktiver Durchmesser: $0.2 \mathrm{~mm}$ ). Der Pumpstrahl wird vorher durch eine Filterkombination (Schott, UG11 und GG454) geblockt. Die Avalanche-Photodiode ist zur Signalverarbeitung an einen Lock-In Verstärker angeschlossen (siehe Kapitel 3.3.3). Um eine „Bühnenkinetik“ zu vermeiden, müssen die beiden Strahlen exakt parallel zueinander verlaufen. Für diese Justage sind die Irisblenden I2-I8 vorgesehen. Die Blende I8 befindet sich in drei Meter Entfernung von der Verzögerungsbühne und wird mit einer Videokamera aufgenommen und abgebildet. Dies erleichtert die Justage über größere Entfernungen.

Die Messungen wurden alle im magischen Winkel und bei $298 \mathrm{~K}$ durchgeführt. Für alle weiteren Messbedingungen sei auf die entsprechenden Kapitel verwiesen. 


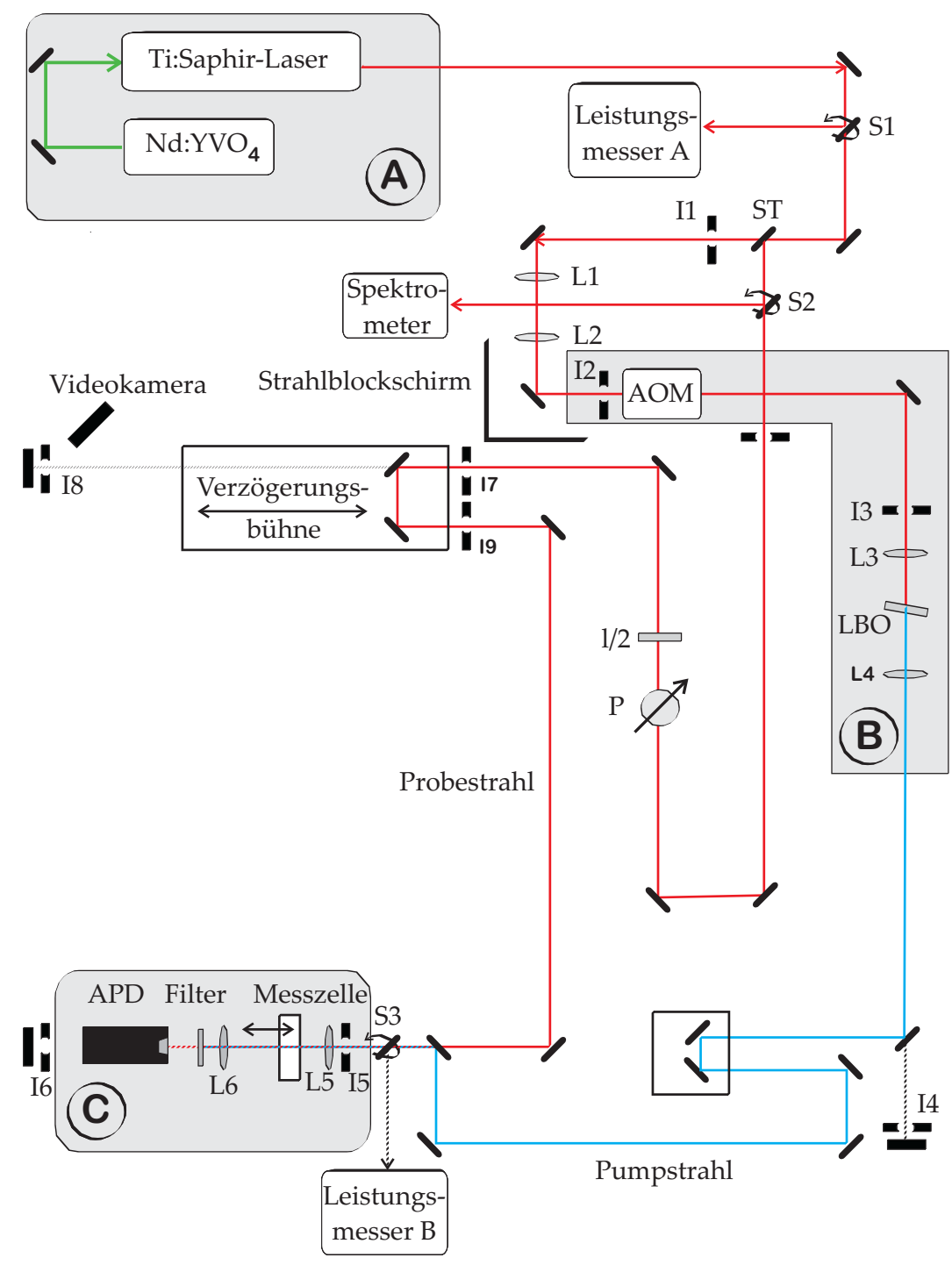

Abbildung 3.6: Experimenteller Aufbau des ultraschnellen transienten Absorptionsexperiments. Das Lasersystem (A) besteht aus einem Nd:YVO 4 -Laser (Millenia Xs, Spectra-Physics) gepumpten Ti:Saphir-Laser (Tsunami, Spectra-Physics). (B) zeigt den Teil zur Erzeugung der Pumppulse und (C) stellt die Einheit der Signalerzeugung und Datenaufnahme dar. Die Abkürzungen werden im Text erläutert.

\subsubsection{Das Tsunami-System}

Die ionischen Flüssigkeiten sind in diesem Experiment mit einem Titan-Saphir-Oszillator (Tsunami) der Firma Spectra-Physics untersucht worden. Als Pumplaser kommt ein Nd:$\mathrm{YVO}_{4}$ Laser (Millenia Xs, Spectra-Physics) zum Einsatz. Das Tsunami-System liefert bei 860 nm Pulse mit einer Dauer von 120 fs und einer Repititionsrate von $82 \mathrm{MHz}$. Der Millenia hat eine Ausgangswellenlänge von 1064 nm die nach einer „Intracavity“-Frequenzverdop- 
pelung $532 \mathrm{~nm}$ beträgt, die Leistung des Lasers wird auf $8.5 \mathrm{~W}$ eingestellt. Die Leistung des Ti:Saphir-Oszillators beträgt im Mittel 1.4 W. Den schematischen Aufbau des Ti:SaphirLasers zeigt Abbildung 3.7

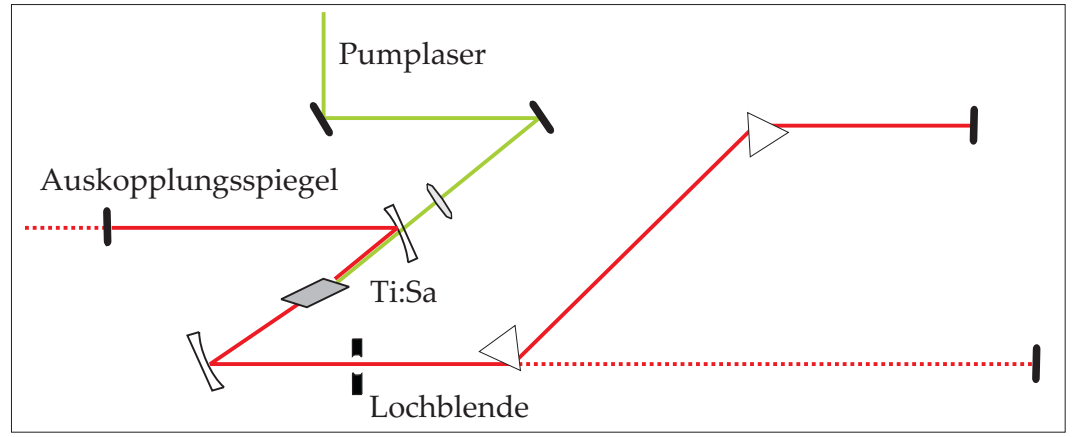

Abbildung 3.7: Schematischer Aufbau des Titan-Saphir Lasers.

Im Lasermaterial ist der Saphir $\left(\mathrm{Al}_{2} \mathrm{O}_{3}\right)$ mit Ti $^{3+}$ dotiert. Aus der Kristallfeldtheorie folgt eine Aufspaltung der d-Orbitale des Titans in $\mathrm{e}_{g}$ - und $\mathrm{t}_{2 g}$-Orbitale. Diese Aufspaltung beträgt etwa $20000 \mathrm{~cm}^{-1}$ respektive $500 \mathrm{~nm}$. Nach Anregung der $\mathrm{Ti}^{3+}$-Ionen wird das obere $\mathrm{e}_{g}$-Niveau erneut aufgespalten. Dies führt zu einer energetischen Absenkung des elektronisch angeregten Zustands, $\mathrm{E}_{g} \rightarrow \mathrm{A}_{1 g}+\mathrm{B}_{1 g}$. Das Prinzip, welches dem zugrunde liegt, wird als Jahn-Teller-Effekt bezeichnet. Die überschüssige Energie wird durch Gitterschwingungen abgeführt, sodass sich ein neues Gleichgewicht einstellt. Der Ti-Saphir-Oszillator ist ein Vier-Niveau-Laser, wie in Abbildung 3.8 ersichtlich wird.

Ti:Saphir-Laser haben den Vorteil eines großen Abstimmbereichs von etwa $300 \mathrm{~nm}$. Als Begründung hierfür ist die einzigartige Energieniveaustruktur von Titan zu nennen. Aufgrund der $3 \mathrm{~d}^{1}$-Konfiguration ist eine Selbstabsorption von Laserstrahlung unterbunden. In anderen Festkörperlasern schränkt diese Selbstabsorption den Abstimmbereich stark ein. Die Absorptionsbande des Ti:Saphir liegt im blau-grünen Bereich des sichtbaren Spektrums, wohingegen die Fluoreszenzbande gut separiert bei etwa 690 bis $1080 \mathrm{~nm}$ zu finden ist.

Femtosekundenpulse können nicht einfach durch Zufuhr gepulster Pumpenergie oder mit Hilfe von Güteschaltungen (Q-Switches) erzeugt werden. Viele longitudinale Moden mit fester Phasenbeziehung müssen im Resonator überlagert werden, dies wird im Fall des Ti:Saphir-Lasers durch eine Kerr-Linsen-Modenkopplung erzielt. Die Repetitionsrate eines Lasers ergibt sich aus der reziproken Umlaufdauer im Resonator, die Halbwertsbreite 


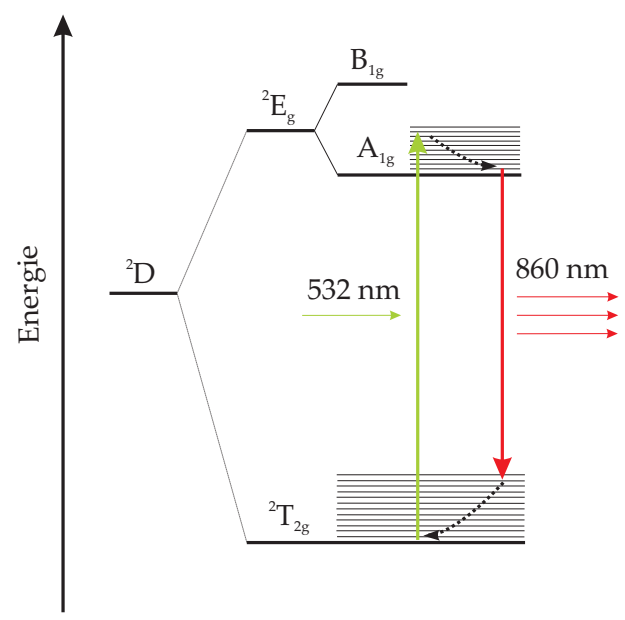

Abbildung 3.8: Vier Niveau-System eines Titan-Saphir Kristalls

entspricht ungefähr der reziproken Verstärkungsbandbreite $\tau \approx \frac{1}{\Delta \nu}$.

Ist das Verstärkungsprofil breit, können viele Moden anschwingen, wodurch ein kurzer Puls erzeugt werden kann. Das Prinzip der Kerr-Linsen-Modenkopplung beruht auf der Selbstfokussierung des Pumpstrahls im Medium. Hierbei handelt es sich um einen nichtlinearen Effekt wie schon oben besprochen worden ist. Die schwächer fokussierten Anteile (cw-Moden) werden durch eine Lochblende geblockt, sodass nur der modengekoppelte Puls verstärkt wird. Eine Kombination von Prismenpaaren komprimiert die Pulse um die Gruppengeschwindigkeitsdispersion im Resonator zu kompensieren. Weitergehende Informationen zum Thema Ti:Saphir-Laser ist in der Dissertation von KAPPEL zu finden. 85

\subsubsection{Messzelle}

Die transienten Absorptionsspektren wurden in einer Durchflussküvette (Hellma) mit $1 \mathrm{~mm}$ Weglänge und $1.25 \mathrm{~mm}$ dicken Fenstern, wie in Abbildung 3.9 zu sehen, aufgenommen. Hierbei handelt es sich um eine Spezialanfertigung von der Firma Hellma. Die Messzelle kann zusätzlich durch einen weiteren Kreislauf mit Hilfe eines Thermostaten auf $25^{\circ} \mathrm{C}$ temperiert werden. Der Durchfluss der ionischen Flüssigkeiten bzw. deren Mischungen mit organischen Lösungsmitteln wird durch eine Mikropumpe gewährleistet. Die Abbildung weicht etwas von der tatsächlich verwendeten Messzelle ab, verdeutlicht aber das Prinzip besser. 


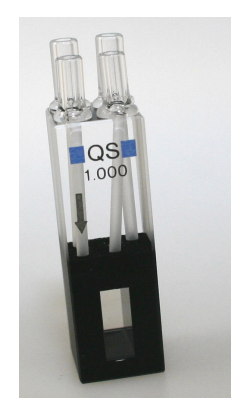

Abbildung 3.9: Temperierbare Durchflussküvette. Fotografiert von Andrea Kempf mit freundlicher Genehmigung von Hellma.

\subsubsection{Datenaufnahme}

Das Prinzip der Datenaufnahme ist in Abbildung 3.10 veranschaulicht. Die Intensität des Pumppulses wird durch einen akusto-optischen Modulator (AOM) moduliert. Daher ist dann auch die transiente Absorption bzw. stimulierte Emission der molekularen Sonden nach der Anregung intensitätsmoduliert. Das Signal wird von einer Avalanche-Photodiode (APD) detektiert und an einen Lock-In-Verstärker (Stanford Research Systems, SR 844) gesendet. Dieser bekommt ein Referenz-Signal (2 MHz) von einem Pulsgenerator, der gleichzeitig den AOM antreibt. Die analogen Signale werden $300 \mathrm{~ms}$ lang gemittelt und durch einen 12-Bit-A/D-Wandler (Data Translation, DT3003PGL) transformiert. Mit Hilfe einer Messsoftware werden die Signale verwertet, dieses Programm steuert zugleich die Verzögerungsbühne.

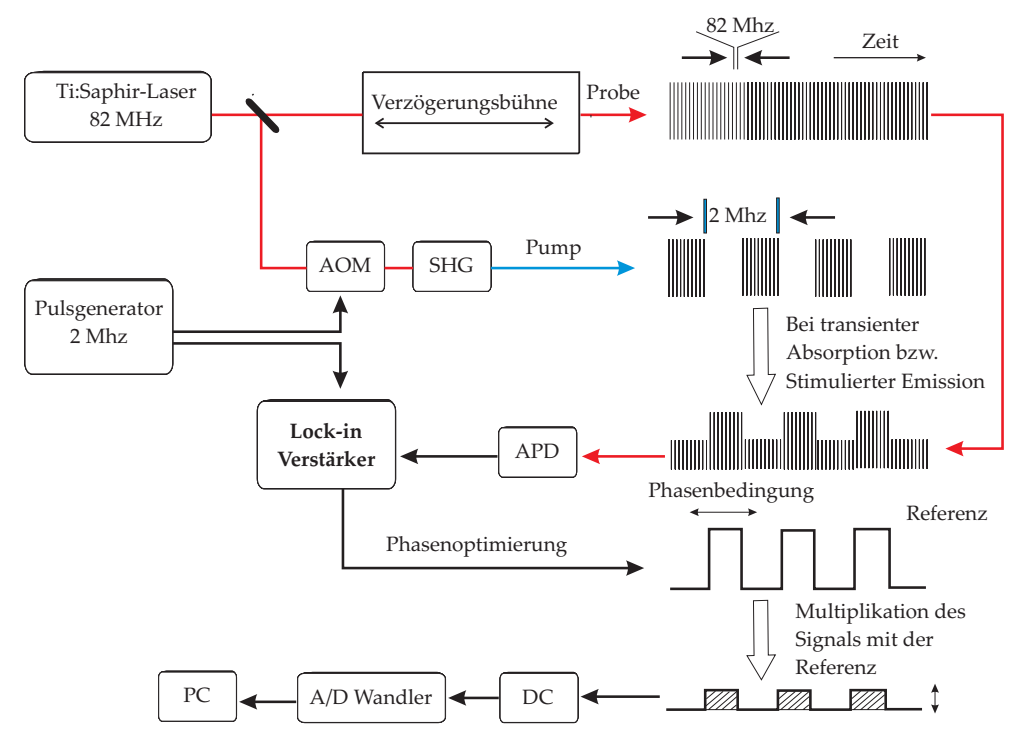

Abbildung 3.10: Prinzip der Datenaufnahme im ultraschnellen TA-Experiment. 
Der Lock-In-Verstärker ermöglicht es, sehr schwache Signale zu messen. Im Prinzip wird hierbei das Rauschen aus den Signalen herausgefiltert. In Abbildung 3.11 ist der prinzipielle Aufbau stark vereinfacht dargestellt. Signal und Referenz werden miteinander multipliziert und in einem Tiefpass integriert. Diese Kreuzkorrelation ermöglicht es, alle Signale, die nicht der Referenz ( $2 \mathrm{MHz}$ ) entsprechen, zu verwerfen. Im Lock-In lässt sich die Phasenbeziehung zwischen Referenz und Signal einstellen, sodass ein möglichst großes Signal gefunden werden kann.

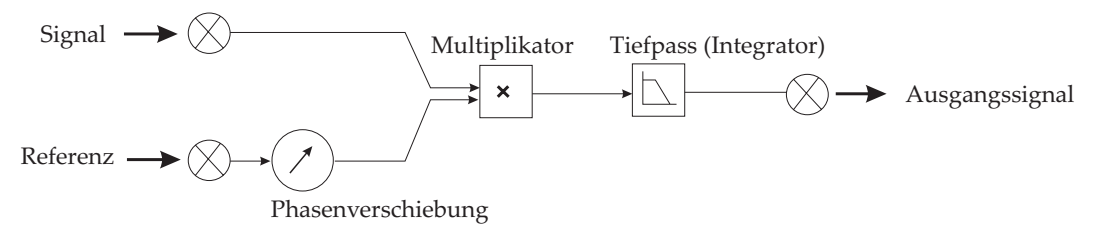

Abbildung 3.11: Prinzipieller Aufbau des Lock-In-Verstärkers.

\subsection{UV/Vis-spektroskopische Untersuchungen: Solvatochromie}

Die molekularen Sonden wurden unter anderem in Mischungen von ionischen Flüssigkeiten mit Acetonitril UV/Vis-spektroskopisch untersucht. Die Verschiebung der Absorptionsbande in Abhängigkeit vom Lösungsmittel bzw. von der Zusammensetzung der binären Mischungen, auch Solvatochromie genannt, steht bei diesen Experimenten im Mittelpunkt. Die Lage der Absorptionsmaxima hängt von der Polarisierbarkeit des Lösungsmittels ab, daher wurden die Brechzahlen aller binären Mischungen gemessen, um eine Korrelation mit dem Absorptionsmaximum herstellen zu können (siehe Kapitel 5.2.1)

Die UV/Vis-Spektren wurden bei Zimmertemperatur über einen Wellenlängenbereich von 200 bis $800 \mathrm{~nm}$ aufgenommen. Es wurde ein Spektrometer der Firma Varian (Cary 5E) benutzt. Beispielspektren aller verwendeten molekularen Sonden sind in Abbildung $3.12 \mathrm{zu}$ sehen, die Auflösung beträgt $0.5 \mathrm{~nm}$. Mit D gekennzeichnete Moleküle stellen hierbei die indolinbasierten Farbstoffe dar, 12'CA und 12'TAC sind Carotinoide. Eine genaue Zuordnung der Abkürzungen erfolgt im nächsten Kapitel. Für die SolvatochromieUntersuchungen der binären Mischungen von ionischen Flüssigkeiten und Acetonitril wurde $12^{\prime} \mathrm{CA}$ als Sonde verwendet. 


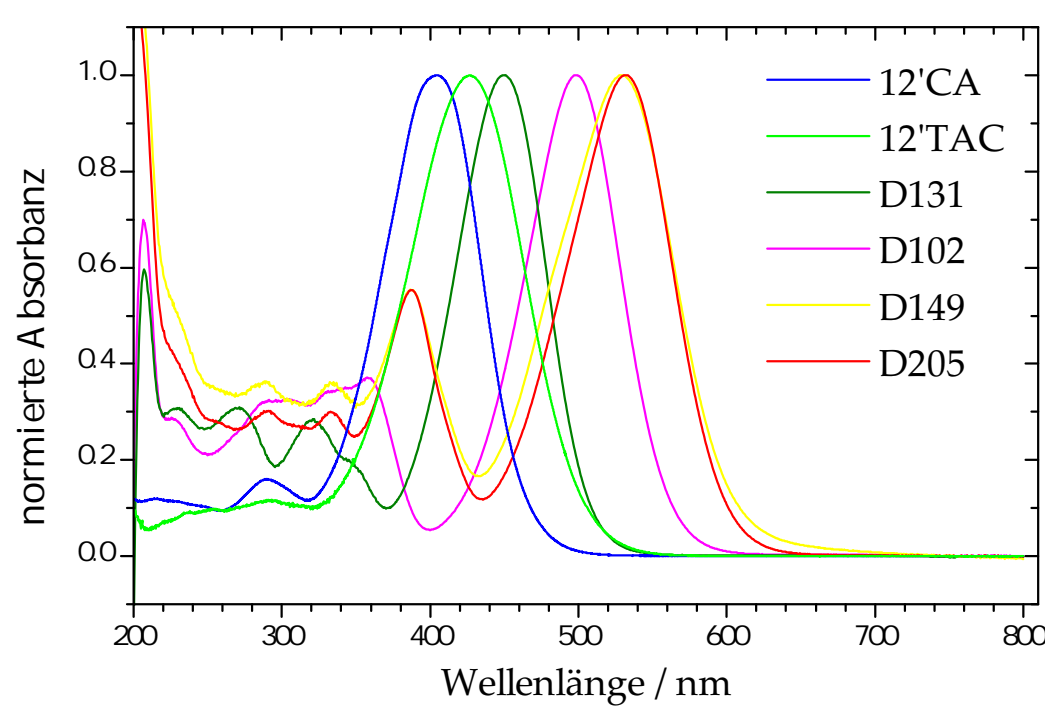

Abbildung 3.12: Stationäre Spektren der verwendeten molekularen Sonden in Methanol.

\subsection{Chemikalien}

Die molekularen Sonden 12'-Apo- $\beta$-carotin-12'-säure (12'CA) und 12'-Apo- $\beta$-carotin-12'-al (12'TAC) sind als reine Proben von der BASF AG zur Verfügung gestellt worden. Die indolinbasierten Farbstoffe D102, D131, D149 und D205 sind von der Firma Mitsubishi Paper Mills bezogen worden. Alle molekularen Sonden werden unter Argonatmosphäre aufbewahrt. Die Carotinoide müssen bei $252 \mathrm{~K}$ gelagert werden, wohingegen die indolinbasierten Sonden bei Zimmertemperatur verweilen können. Aufgrund der Hygroskopie der ionischen Flüssigkeiten werden alle Proben für die Experimente in einer Glove-Box vorbereitet, als Inertgas wird auch hier Argon verwendet. Als zusätzliche Maßnahme um den Wassergehalt gering zu halten, werden die ionischen Flüssigkeiten unter Molekularsieb (Merck, Porengröße 3) aufbewahrt. Eine Zusammenfassung aller verwendeten Chemikalien ist in Tabelle 3.1 gegeben. Die Struktur der molekularen Sonden ist in Abbildung 3.13 zu sehen. Für die Strukturformeln der ionischen Flüssigkeiten sei auf die nachfolgenden Kapitel verwiesen. 
Tabelle 3.1: Chemikalien

\begin{tabular}{|c|c|c|c|}
\hline Substanz & Hersteller & Reinheit [\%] & Molmasse $[\mathrm{g} / \mathrm{mol}]$ \\
\hline \multicolumn{4}{|l|}{ molekulare Sonden } \\
\hline $12^{\prime} \mathrm{CA}$ & BASF & $>97$ & 366.5 \\
\hline 12'TAC & BASF & $>97$ & 350.5 \\
\hline D102 & Mitsubishi Paper Mills Ltd. & $>95$ & 614.8 \\
\hline D131 & Mitsubishi Paper Mills Ltd. & $>95$ & 508.6 \\
\hline D149 & Mitsubishi Paper Mills Ltd. & $>95$ & 741.9 \\
\hline D205 & Mitsubishi Paper Mills Ltd. & $>95$ & 826.1 \\
\hline \multicolumn{4}{|l|}{ ionische Flüssigkeiten } \\
\hline$\left[\mathrm{C}_{6} \mathrm{mim}\right]^{+}\left[\mathrm{Tf}_{2} \mathrm{~N}\right]^{-}$ & IoLiTec & 99 & 447.4 \\
\hline$\left[\mathrm{C}_{2} \mathrm{mim}^{+}\left[\mathrm{EtSO}_{4}\right]^{-}\right.$ & IoLiTec & 99 & 236.3 \\
\hline$\left[\mathrm{C}_{4} \mathrm{mim}\right]^{+}\left[\mathrm{Tf}_{2} \mathrm{~N}\right]^{-}$ & IoLiTec & 99 & 419.4 \\
\hline$\left[\mathrm{C}_{4} \mathrm{mmim}\right]^{+}\left[\mathrm{Tf}_{2} \mathrm{~N}\right]^{-}$ & IoLiTec & 99 & 433.4 \\
\hline$\left[\mathrm{C}_{2} \mathrm{mim}\right]^{+}\left[\mathrm{N}(\mathrm{CN})_{2}\right]^{-}$ & IoLiTec & $>98$ & 177.2 \\
\hline$\left[\mathrm{C}_{4} \mathrm{mim}\right]^{+}\left[\mathrm{B}(\mathrm{CN})_{4}\right]^{-}$ & Merck & $>99$ & 257.2 \\
\hline \multicolumn{4}{|c|}{ organische Lösungsmittel } \\
\hline Methanol & Merck & $>99.9$ & 32.0 \\
\hline Ethanol & Merck & $>99.9$ & 46.1 \\
\hline Acetonitril & Merck & $>99.5$ & 41.1 \\
\hline Tetrahydrofuran & Merck & $>99.9$ & 72.1 \\
\hline
\end{tabular}<smiles>CC(C=CCCC(C)=CCCC=C(C)C(=O)O)=CC=CC1=C(C)CCCC1(C)C</smiles>

12'CA<smiles>O=C(O)CN1C(=O)/C(=C\c2ccc3c(c2)C2CCCC2N3c2ccc(C=C(c3ccccc3)c3ccccc3)cc2)SC1=S</smiles>

D102<smiles>CC(=O)/C=C(\C)CC/C=C/C(C)=C/C=C/C=C(C)/C=C/C1=C(C)CCCC1(C)C</smiles>

12'TAC<smiles>N#C/C(=C\c1ccc2c(c1)C1CCCC1N2c1ccc(C=C(c2ccccc2)c2ccccc2)cc1)C(=O)O</smiles>

D131<smiles>CCN1C(=O)/C(=c2\s/c(=C/c3ccc4c(c3)C3CCCC3N4c3ccc(C=C(c4ccccc4)c4ccccc4)cc3)c(=O)n2CC(=O)O)SC1=S</smiles>

D149

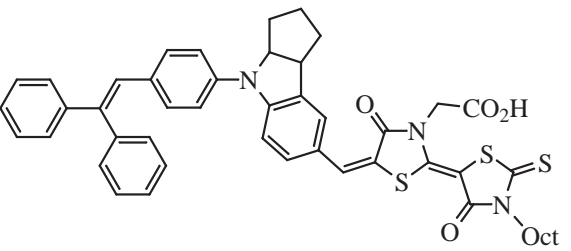

D205

Abbildung 3.13: Chemische Strukturformeln der molekularen Sonden. 


\section{Kapitel 4}

\section{Apocarotinoide zur Untersuchung lokaler Wechselwirkungen in ionischen Flüssigkeiten}

Carotinoide sind bekannt für ihre faszinierende Farbenpracht. Im Herbst geben sie den Blättern die schönen Rot- und Gelbtöne. Sie sind zusammen mit Chlorophyll die am häufigsten in der Natur vorkommenden Farbpigmente. Als Lichtsammelpigmente decken sie einen Bereich des sichtbaren elektromagnetischen Spektrums ab, den Chlorophylle nicht erreichen können und spielen damit unter anderem eine wichtige Rolle in der Photosynthese. Durch oxidative Spaltung der Carotinoide entstehen so genannte Apocarotinoide, unter ihnen die besondere Klasse mit konjugierter Carbonylfunktion. Ein berühmter Vertreter dieser Klasse ist Retinal, eine Form des Vitamin A. Die am meisten untersuchte Funktion dieser Carbonyl-Carotinoide ist die des Lichtsammelns in einigen marinen Algen. ${ }^{9495}$

Die Photophysik von carbonylsubstituierten Carotinoiden hat in den letzten Jahren beträchtliche Aufmerksamkeit erregt. Der erste angeregte Zustand dieser Apocarotinoide zeigt in einigen Fällen eine starke Abhängigkeit der Lebensdauer von der Polarität des Lösungsmittels. $\frac{79[809496699}{9}$ Es wird angenommen, dass dieser Zustand intramolekularen Charge-Transfer (ICT)-Charakter aufweist, daher wird er als $\mathrm{S}_{1} /$ ICT bezeichnet. Die Beschaffenheit dieses Zustands wird immer noch diskutiert. Bei dieser Debatte geht man auf der einen Seite davon aus, dass es zwei separierte Zustände $S_{1}$ und ICT gibt, die miteinander gekoppelt sind und auf der anderen Seite wird ein einzelner Zustand $S_{1}$ mit ICTCharakter diskutiert. $100+104$ 
<smiles>CC1=C(/C=C/C(C)=C/C=C/C(C)=C/C=C/C=C(\C)C=O)C(C)(C)CCC1</smiles>

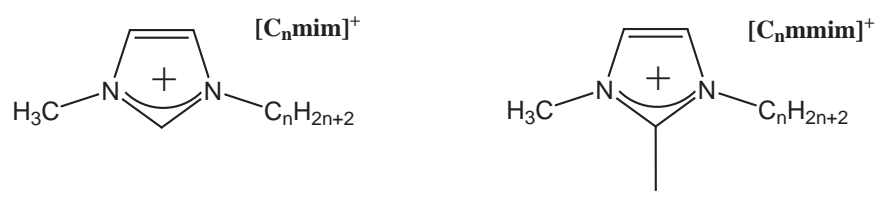<smiles>O=S(=O)(NS(=O)(=O)C(F)(F)F)C(F)(F)F</smiles>

Abbildung 4.1: Chemische Strukturformeln.

In diesem Kapitel werden Ergebnisse der ultraschnellen laserspektroskopischen Experimente an 12'-Apo- $\beta$-carotin-12'-säure (12'CA) und 12'-Apo- $\beta$-carotin-12'-al (12'TAC) in ionischen Flüssigkeiten (ILs) gezeigt (siehe Abbildung 4.1). Diese beiden Farbstoffe eignen sich hervorragend für die Untersuchung der Mikropolarität und Solvatationsdyna-

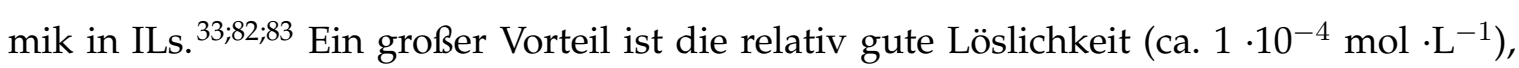
welche besser ist als die von $\beta$-Carotin. Außerdem kann die Bildung von Aggregaten der Carotinoide vermieden werden, welche die Interpretation der Dynamik angeregter Zustände verkomplizieren würde. Der entscheidende Vorteil ist allerdings die relativ lange Lebensdauer des $\mathrm{S}_{1}$ /ICT-Zustands, die eine genaue Analyse der Solvatationsdynamik in ILs bis hin zu größeren Zeitskalen ermöglicht. Es wurden imidazoliumbasierte ILs mit Bis(trifluoromethylsulfonyl)imid $\left[\mathrm{Tf}_{2} \mathrm{~N}\right]^{-}$als Anion betrachtet. Diese Klasse der ILs hat den Vorteil, dass es weniger Probleme mit Wasserverunreinigungen gibt und keine Hydrolyseprodukte wie $\mathrm{HF}$ aus $\left[\mathrm{PF}_{6}\right]^{-}$und $\left[\mathrm{BF}_{4}\right]^{-}$entstehen können. ${ }^{105}$ 


\subsection{Ultraschnelle intramolekulare Prozesse von Apocarotinoiden}

Der elektronische Grundzustand $\left(\mathrm{S}_{0}\right)$ der Carotinoide weist unter Annahme von $\mathrm{C}_{2 h}$-Symmetrie des Polyens $\mathrm{A}_{g}{ }^{-}$-Symmetrie auf. ${ }^{106}$ Der Übergang in den ersten angeregten elektronischen Zustand $\left(\mathrm{S}_{1}\right)$ ist auf Grund seiner $2^{1} \mathrm{~A}_{g}{ }^{-}$-Symmetrie durch 1-Photon-Anregung optisch verboten. Die Anregung in den zweiten elektronisch angeregten Zustand $\left(\mathrm{S}_{2}\right)$ mit ${ }^{1} \mathrm{~B}_{u}{ }^{+}$-Symmetrie ist hingegen erlaubt. Der $\mathrm{S}_{0} \rightarrow \mathrm{S}_{2}$-Übergang bei Carotinoiden besitzt einen hohen Extinktionskoeffizienten von etwa $10^{5} \mathrm{~L} \cdot \mathrm{mol}^{-1} \cdot \mathrm{cm}^{-1}$. Im statischen Absorptionsspektrum der betrachteten Apocarotinoide ist eine relativ breite Bande mit Absorptionsmaximum im sichtbaren Bereich zu erkennen, die diesem Übergang zugeordnet werden kann (siehe Abbildung 3.12). Bei höheren Wellenlängen gibt es keine Absorptionsbanden, was ein Indiz für ausschließlich „dunkle“ niedrigere Zustände ist. Eine weitere Absorptionsbande im UV kann vermutlich dem $2{ }^{1} \mathrm{~B}_{u}{ }^{+}$-Zustand zugeordnet werden (siehe Abbildung 3.12).

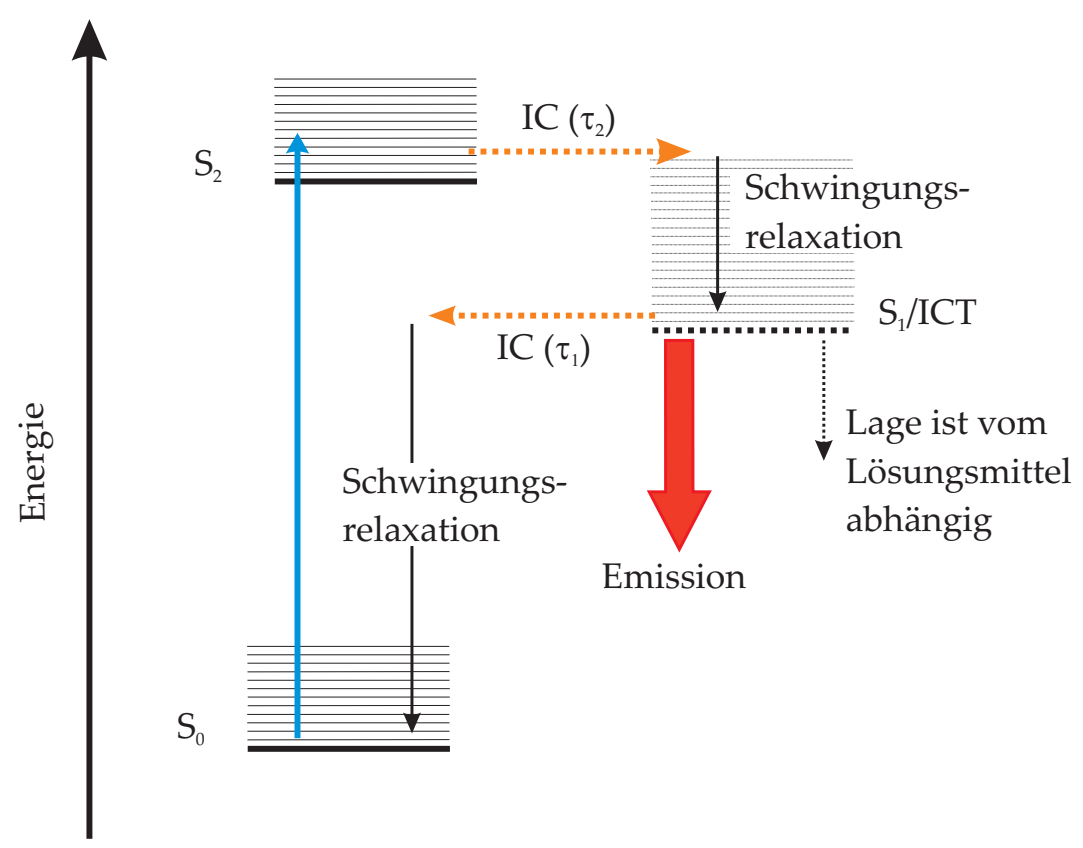

Abbildung 4.2: Drei-Zustände-Modell der Apocarotinoide.

Der $\mathrm{S}_{1}$-Zustand ist nur durch strahlungslose Prozesse z. B. aus dem $\mathrm{S}_{2}$-Zustand zu erreichen, daher spricht man auch von einem „dunklen“ Zustand. Nach Anregung in den $\mathrm{S}_{2}$-Zustand folgen die in Abbildung 4.2 verdeutlichten Relaxationsprozesse. Die Carbonylfunktion der Apocarotinoide sorgt durch die Beeinflussung der Elektronendichte innerhalb 
des konjugierten Polyens für eine komplexere Relaxation als es z. B. in $\beta$-Carotin der Fall ist. Der Zustand mit ICT-Charakter wird von der Polarität des Lösungsmittels beeinflusst. Diesen Einfluss von z. B. verschiedenen ionischen Flüssigkeiten auf die innere Konversion $\mathrm{S}_{1} / \mathrm{ICT} \rightarrow \mathrm{S}_{0}$ der Apocarotinoide kann man durch zeitaufgelöste Messungen bestimmen. Mit steigender Lösungsmittelpolarität kommt es zu einer stärkeren Stabilisierung des $\mathrm{S}_{1} /$ ICT-Zustands relativ zum Grundzustand, wodurch die Lebensdauer des angeregten Zustands unmittelbar beeinflusst wird. Das „Energy-Gap-Law“ ${ }^{107}$ bietet einen möglichen Ansatz zum Verständnis dieses Einflusses. Die Lebensdauer strahlungsloser Prozesse wird folgendermaßen beschrieben:

$$
\tau=\exp (\gamma \cdot \Delta E)
$$

Dabei ist $\tau$ die Lebensdauer des strahlungslosen Prozesses, $\gamma$ ein Proportionalitätsfaktor und $\Delta E$ die Energiedifferenz zwischen zwei Zuständen. Durch steigende Stabilisierung des $\mathrm{S}_{1} /$ ICT-Zustands relativ zu $\mathrm{S}_{0}$ wird $\Delta E$ kleiner und es resultiert eine Abnahme der Lebensdauer. Diese theoretische Abschätzung entspricht qualitativ den experimentellen Beobachtungen. $\underline{81}$

\subsection{Globale Analyse der PSCP-Daten}

In den PSCP-Experimenten werden die transienten Signale simultan für verschiedene Wellenlängen gemessen. In erster Linie ist für diese Arbeit die Relaxation des $\mathrm{S}_{1}$ / ICT-Zustands von Interesse. Die energetische Lage dieses Zustands relativ zum Grundzustand hängt von der Polarität des verwendeten Lösungsmittels ab. Die Untersuchung der Dynamik dieses Zustands ermöglicht die Erkundung der mikroskopischen Umgebung der Sonde 12'CA. In Abschnitt 4.1 ist das Relaxationsschema dieser Sonde präsentiert worden. In Abbildung 4.5 ist beispielsweise im unteren Feld die Relaxation des $\mathrm{S}_{1}$ / ICT-Zustands als Abnahme der Intensitäten der ESA-Banden zu erkennen. Wird nur eine Wellenlänge betrachtet so ergibt sich aus den transienten Spektren eine kinetische Spur. Aus dem langsamen exponentiellen Abfall dieser kinetischen Spur lässt sich durch Anpassung des Signals die Lebensdauer des $\mathrm{S}_{1}$ /ICT-Zustands bestimmen. Dies ist für jede Wellenlänge möglich, die zwischen 350 bis $770 \mathrm{~nm}$ gemessen worden ist. In der vorliegenden Arbeit wurden die Lebensdauern in den unterschiedlichen ionischen Flüssigkeiten durch eine globale Analyse ermittelt. Dafür 
wurden etwa 35 kinetische Spuren mit einem Abstand von ca. $8 \mathrm{~nm}$ entlang des gemessenen Spektrums ausgewählt und mit gleicher Zeitkonstante durch eine Funktion angepasst. Diese Anzahl ist ausreichend um die transienten Spektren zu rekonstruieren und ebenfalls ein gutes Maß für die Auswertbarkeit mit dem Programm Origin 8.

Bei der genauen Analyse der transienten Spektren hat sich ergeben, dass eine biexponentielle Anpassung die kinetischen Spuren am besten widerspiegelt. Dies kann in Abbildung 4.3 nachvollzogen werden. Die kinetischen Spuren wurden aus drei verschiedenen Messreihen zusammengestellt. Bei jeder ionischen Flüssigkeit bzw. bei jedem Lösungsmittel sind drei Scans mit unterschiedlichen Zeitintervall durchgeführt worden. Neben der Messung der reinen ionischen Flüssigkeit von -1 bis 2 ps im 10-fs-Intervall zur Chirp-Korrektur (siehe Kapitel 3.2.4) wird eine Messung der Sonde im gleichen Zeitintervall durchgeführt. Um die gesamte Dynamik abzudecken werden Messungen mit längerem Intervall von 1 bis 5 ps je nach Sonde und Lösungsmittel aufgenommen. Für das Erfassen von mehr Datenpunkten bei früheren Zeiten wird noch ein Scan mit mittlerem Zeitintervall durchgeführt, in dem Fall zwischen 200 fs und 1 ps. Aufgrund von leicht schwankender Laserleistung müssen die drei Scans normiert werden und können dann zu einer kinetischen Spur zusammengefügt werden. In Abbildung 4.3 sind zwei kinetische Spuren abgebildet, die auf drei unterschiedliche Arten simuliert werden. Es sind eine monoexponentielle und eine biexponentielle Anpassung gezeigt. Zusätzlich gibt es eine Anpassung nach dem kinetischen Modell $\mathrm{S}_{2} \rightarrow \mathrm{S}_{1} / \mathrm{ICT} \rightarrow \mathrm{S}_{0}$ (siehe Gleichung 5.8). Die monoexponentielle Anpassung und das kinetische Modell liefern in diesem Beispiel beide die gleiche Lebensdauer $\tau_{1}$. Durch die biexponentielle Anpassung ergibt sich eine langsame und eine schnellere Komponente. Die PSCP-Daten dieser Arbeit werden alle mit einer biexponentiellen Funktion angepasst.

Bei den transienten Spektren wird ein Verschiebung zu kürzeren Wellenlängen mit der Zeit beobachtet. Zur Demonstration dieser Blauverschiebung wird es in den nachfolgenden Kapiteln an einigen Stellen nötig sein, simulierte Spektren zu zeigen. Die transienten Spektren werden alle mit einer möglichst kleinen Anzahl von Gaußfunktionen angepasst. Aus diesen simulierten Spektren können die Absorptionsmaxima extahiert und gegen die Zeit aufgetragen werden. Aus dieser Auftragung erhält man zwei Zeitkonstanten für die Solvensdynamik wie im nächsten Abschnitt gezeigt werden wird. Hier seien noch ein paar angepasste Beispielspektren gezeigt (siehe Abbildung 4.4 


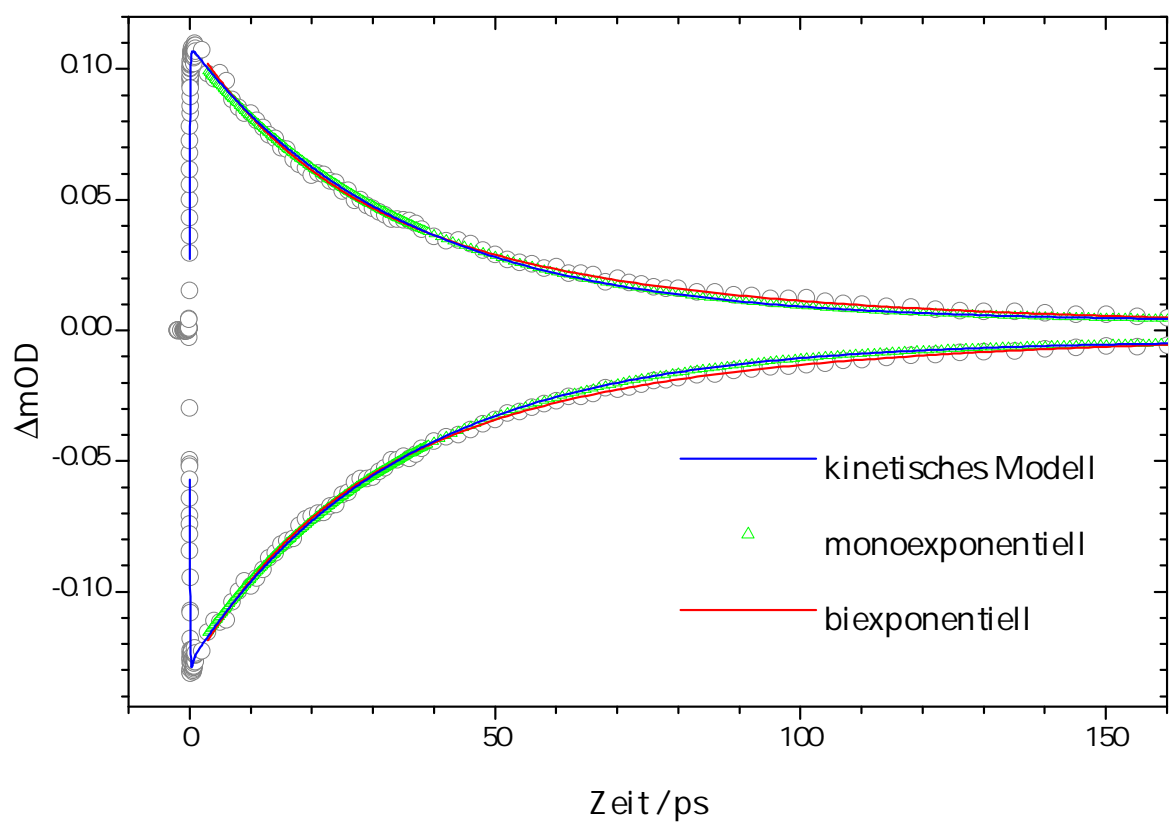

Abbildung 4.3: Verschiedene globale Anpassungen.

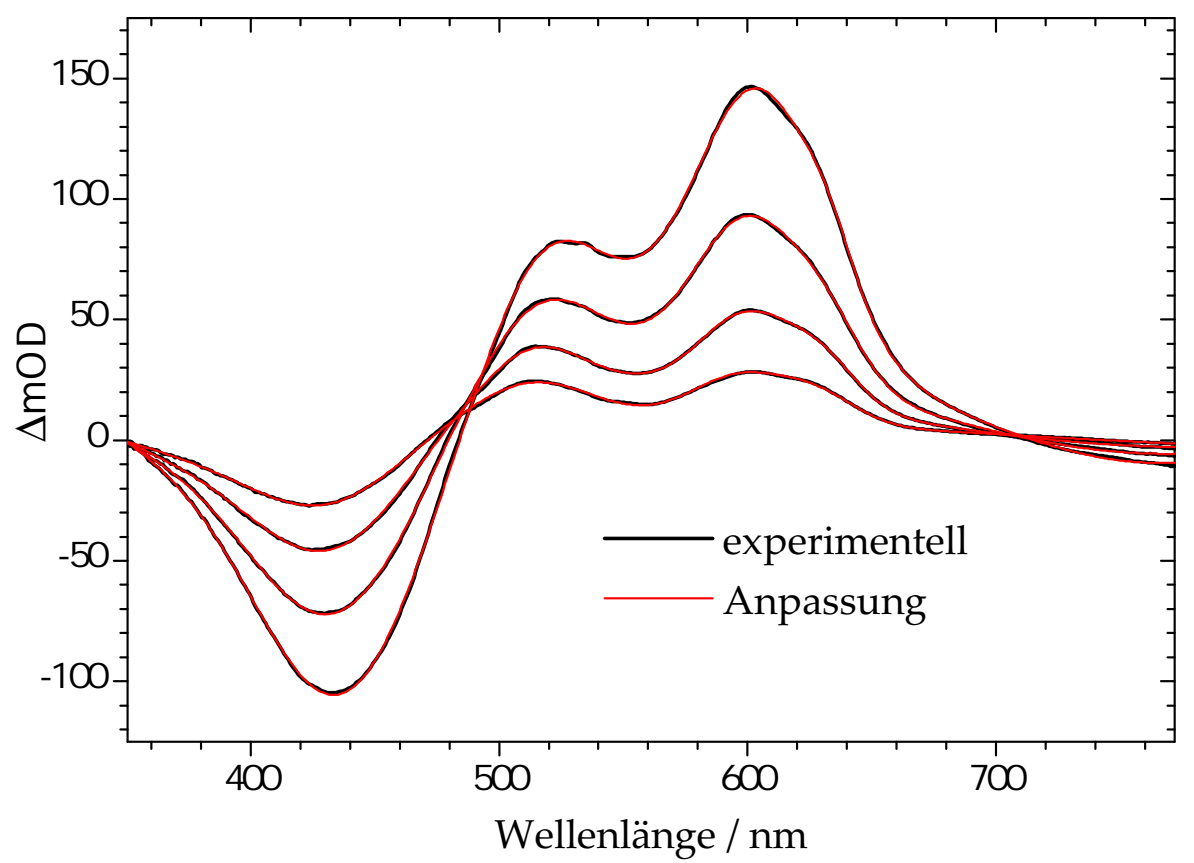

Abbildung 4.4: Mit Gaußfunktionen angepasste transiente Spektren. 


\subsection{Experimentelle Ergebnisse für 12'-Apo- $\beta$-carotin-12'-säure}

Die PSCP-Spektren von $12^{\prime} \mathrm{CA}$ in $\left[\mathrm{C}_{6} \mathrm{mim}\right]^{+}\left[\mathrm{Tf}_{2} \mathrm{~N}\right]^{-},\left[\mathrm{C}_{2} \mathrm{mim}\right]^{+}\left[\mathrm{EtSO}_{4}\right]^{-},\left[\mathrm{C}_{4} \mathrm{mim}\right]^{+}\left[\mathrm{Tf}_{2} \mathrm{~N}\right]^{-}$ und $\left[\mathrm{C}_{4} \mathrm{mmim}\right]^{+}\left[\mathrm{Tf}_{2} \mathrm{~N}\right]^{-}$sind in Abbildung 4.5 bis 4.8 gezeigt. Die molekulare Sonde wird in allen ionischen Flüssigkeiten bei $480 \mathrm{~nm}$ angeregt. Im oberen Abschnitt der Abbildungen ist die Entwicklung bei sehr frühen Zeiten zu sehen. Negative Signale stammen dabei vom Ground-State Bleach (GSB) und der stimulierten Emission (SE), wohingegen die positiven Signale der Excited-State Absorption (ESA), also der Absorption aus den angeregeten Zuständen, zuzuordnen sind. Während der Pumppuls anwächst und sein Maximum bei einer Zeitverzögerung zwischen Pump- und Probepuls von 0 ps erreicht, erscheint der $S_{0} \rightarrow S_{2^{-}}$ GSB und die $S_{2} \rightarrow S_{0}$-SE. Die Struktur auf diesen frühen Signalen stammt von den Ramanübergängen der molekularen Sonde. Die transiente Absorption zwischen 650 und $750 \mathrm{~nm}$ erfolgt aus dem zweiten angeregten Zustand des Carotinoids in einen höher gelegenen Zustand. Diese Absorption verschwindet dann mit der Bildung des $\mathrm{S}_{1} /$ ICT-Zustands.

Im mittleren Abschnitt werden zwei ESA-Banden des $\mathrm{S}_{1} / \mathrm{ICT}$ deutlich. Aus weiteren Messungen von 12 'CA in anderen Lösungsmitteln ist bekannt, dass die breitere Bande bei höheren Wellenlängen aus zwei sich überlappenden Banden bestehen könnte. ${ }^{83}$ Das heißt, die ESA findet wahrscheinlich in drei höher gelegene elektronische Zustände statt. Der Peak bei etwa $750 \mathrm{~nm}$ nimmt mit der Zeit ab, weil hier die ESA aus dem $\mathrm{S}_{2}$-Zustand mit voranschreitener Depopulation abnimmt. Es bleibt allerdings ein Restsignal in diesem Bereich vorhanden, was nicht der $\mathrm{S}_{2}$-ESA zuzuordnen ist, sondern einem Radikalkation der 12'CA. Dieses Radikalkation wird durch Zwei-Photonen Ionisation geformt. An dieser Stelle muss erwähnt werden, dass man in diesem nahen IR Bereich eine schwache stimulierte Emission bei den Apocarotinoiden beobachtet, dies deutet auf einen ICT-Charakter dieser Moleküle hin. $\frac{33|8199| 108 \mid 109}{}$ Das Erscheinen stimulierter Emission in diesem Bereich ist Grundlage für die Zweifarben-TA Messungen, welche in den nachfolgenden Kapiteln gezeigt werden. Im Fall der PSCP-Messungen ist diese SE durch die Absorption des Radikalkations von 12'CA überdeckt.

Der untere Abschnitt der Abbildungen 4.5 bis 4.8 zeigt die Entwicklung der transienten Spektren bei langen Zeiten bis zu mehreren hundert Pikosekunden. Hier wird deutlich, wie die $S_{1} /$ ICT-ESA-Banden abnehmen und gleichzeitig der GSB aufgefüllt wird. Allerdings bleibt ein kleiner Rest des GSB erhalten, da ein Teil der Grundzustandspopulation 
von 12 'CA in Radikalkationen umgewandelt worden ist. Bei den Messungen der ionischen Flüssigkeiten gibt es stetig Probleme mit dem Streulicht des Pumpstrahls, welches in einigen Fällen als zusätzliche Struktur im Signal bei etwa $480 \mathrm{~nm}$ zu erkennen ist. Ebenfalls im unteren Abschnitt gezeigt sind die stationären Absorptionsspektren (gestrichelte Linie) von $12^{\prime} \mathrm{CA}$ in den jeweiligen ionischen Flüssigkeiten.

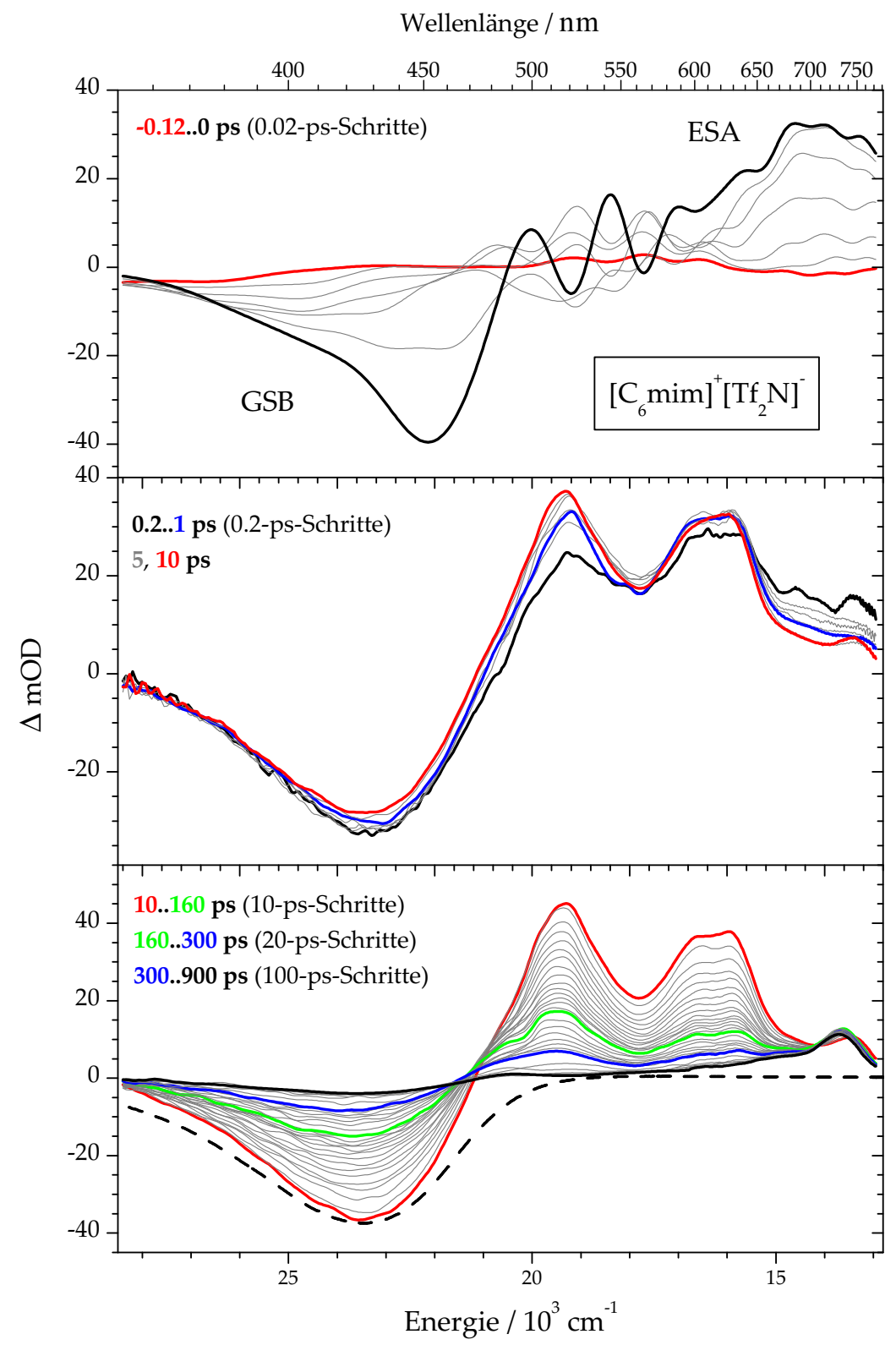

Abbildung 4.5: Transientes PSCP-Absorptionsspektrum von $12^{\prime} \mathrm{CA}$ in $\left[\mathrm{C}_{6} \mathrm{mim}\right]^{+}\left[\mathrm{Tf}_{2} \mathrm{~N}\right]^{-}$bei einer Anregungswellenlänge von $480 \mathrm{~nm}$. $\Delta$ mOD ist die Änderung der optischen Dichte in $10^{-3}$. 


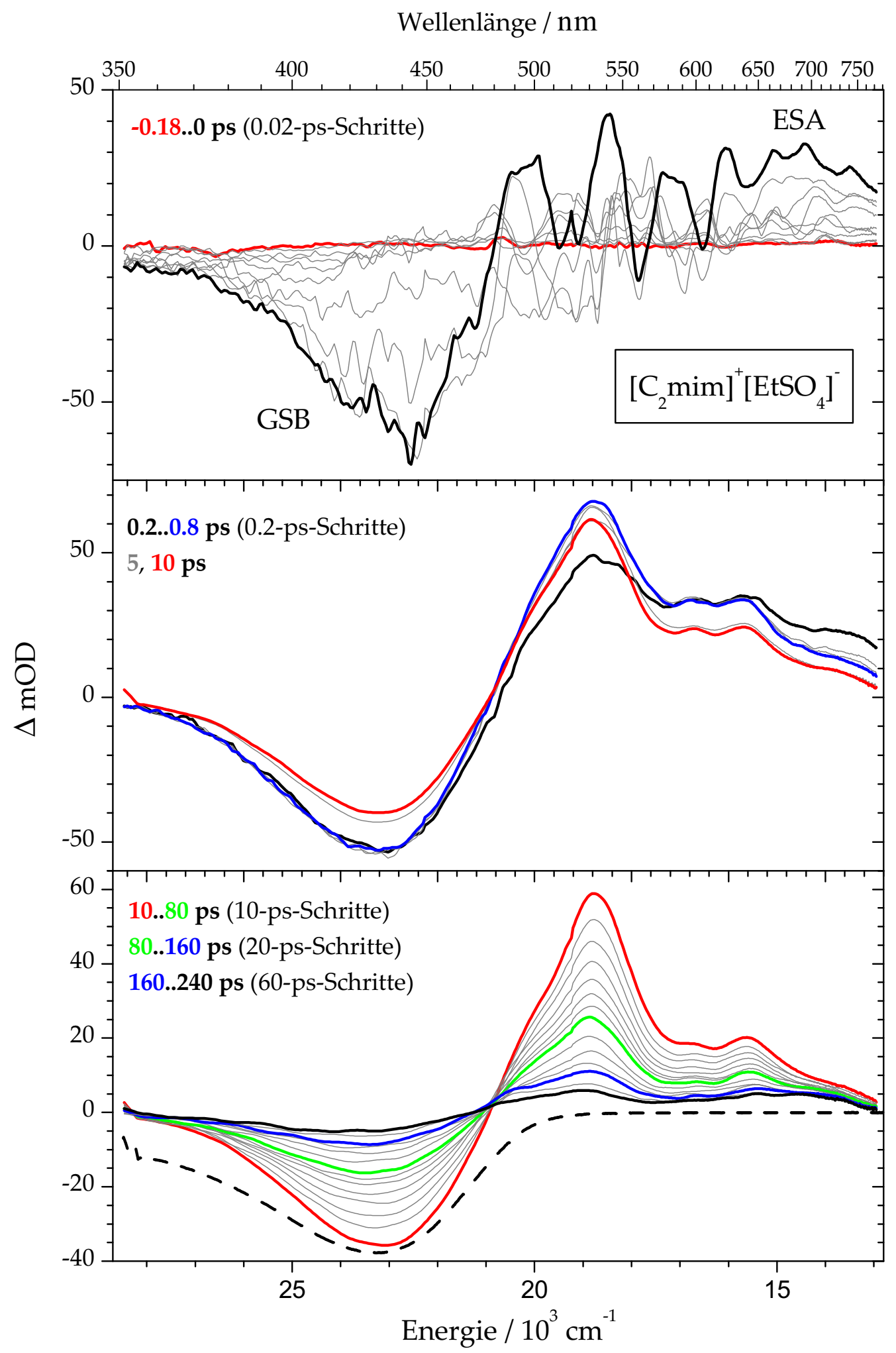

Abbildung 4.6: Wie Abbildung 4.5, aber in $\left[\mathrm{C}_{2} \mathrm{mim}\right]^{+}\left[\mathrm{EtSO}_{4}\right]^{-}$. 


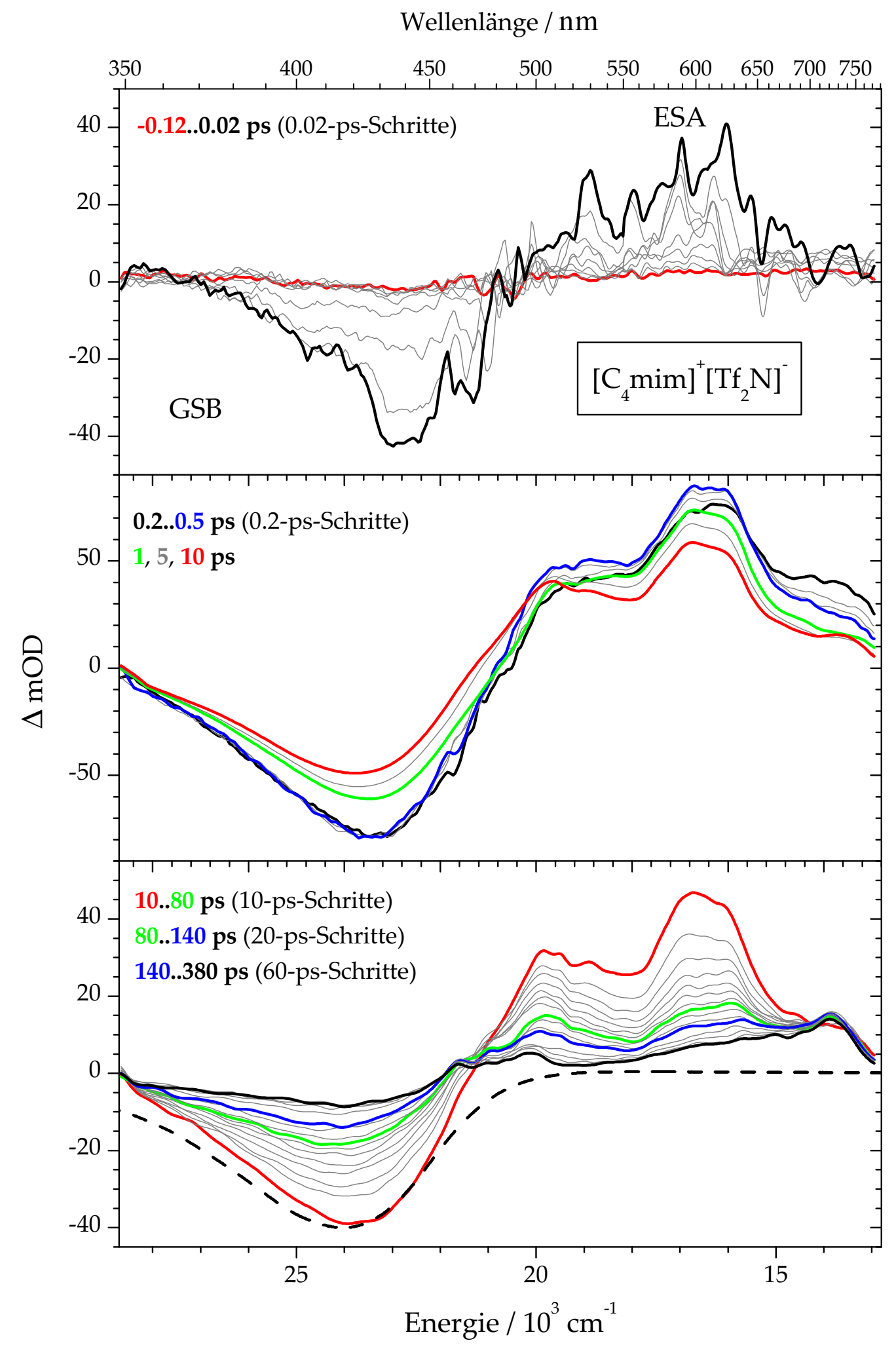

Abbildung 4.7: Wie Abbildung 4.5, aber in $\left[\mathrm{C}_{4} \mathrm{mim}\right]^{+}\left[\mathrm{Tf}_{2} \mathrm{~N}\right]^{-}$. 


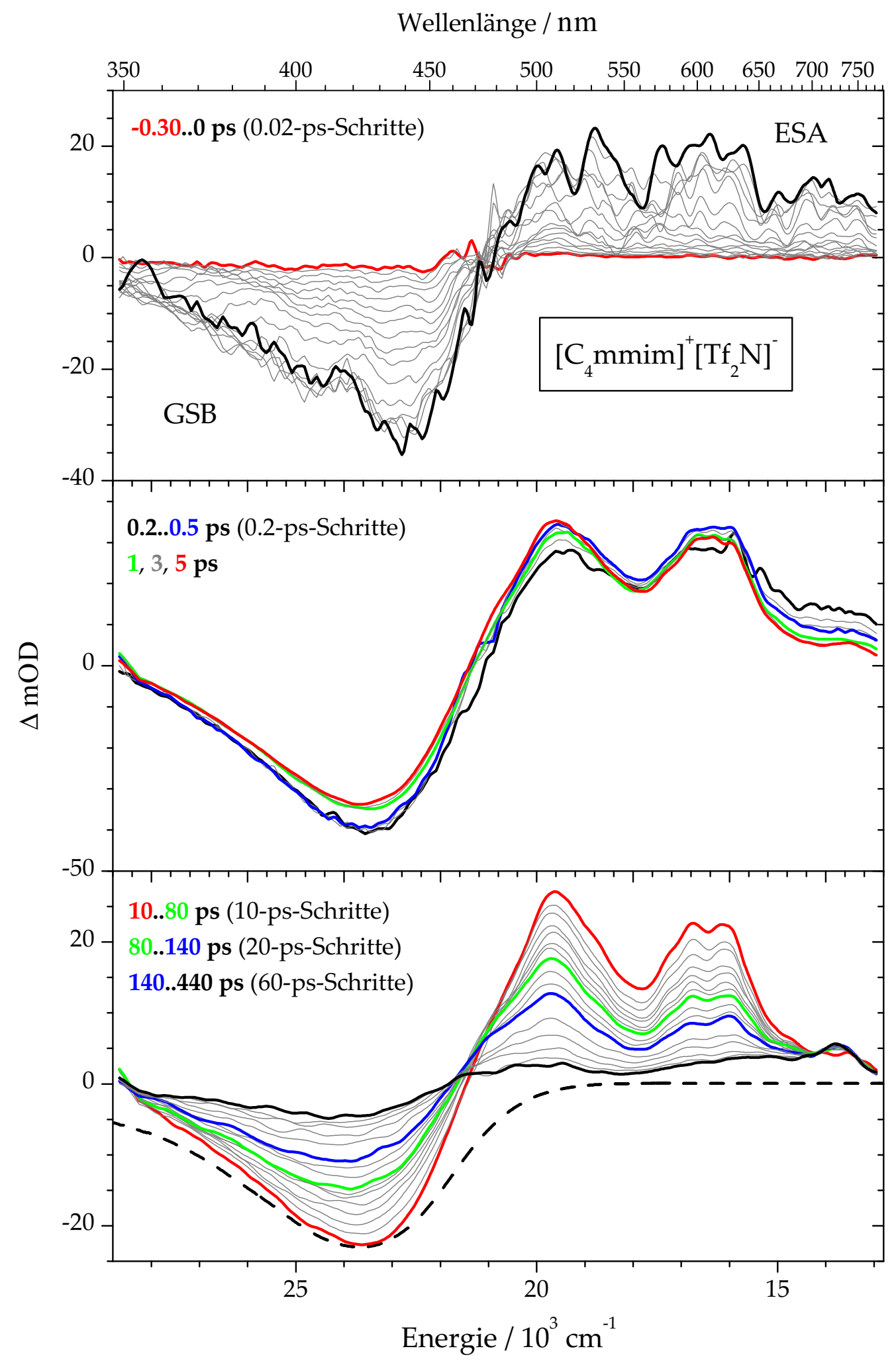

Abbildung 4.8: Wie Abbildung 4.5, aber in $\left[\mathrm{C}_{4} \mathrm{mmim}\right]^{+}\left[\mathrm{Tf}_{2} \mathrm{~N}\right]^{-}$. 
Wie in den vorhergehenden Abbildungen zu erkennen gibt es eine spektrale Verschiebung der Absorptionsmaxima mit der Zeit. Besonders gut ist dies bei der linken ESA-Bande zu sehen. In nachfolgender Abbildung ist ein Ausschnit der transienten ESA-Banden von $12^{\prime} \mathrm{CA}$ in $\left[\mathrm{C}_{6} \mathrm{mim}\right]^{+}\left[\mathrm{Tf}_{2} \mathrm{~N}\right]^{-}$als angepasste Spektren gezeigt, um die Blauverschiebung dieser Bande zu verdeutlichen. In Abbildung 4.10 ist dann exemplarisch die zeitliche Entwicklung des Absorptionsmaximums der ESA-Bande abgebildet. Die Daten sind mit folgender Funktion angepasst:

$$
\nu(t)=\nu_{0}+A_{1} \cdot \exp \left(-\left(\frac{t}{\tau_{l}}\right)^{\beta}\right)+A_{2} \cdot \exp \left(-\frac{t}{\tau_{s}}\right) .
$$

Die Blauverschiebung hervorgerufen durch eine Solvensdynamik lässt sich mit den Zeitkonstanten $\tau_{l}$ für die langsame Komponenten und $\tau_{s}$ für die schnelle Komponenten beschreiben (siehe Tabelle 4.1). Die langsamere Komponente wird hierbei durch eine gestreckte Exponentialfunktion repräsentiert, wohingegen die schnelle Komponente aus dem monoexponentiellen Teil stammt.

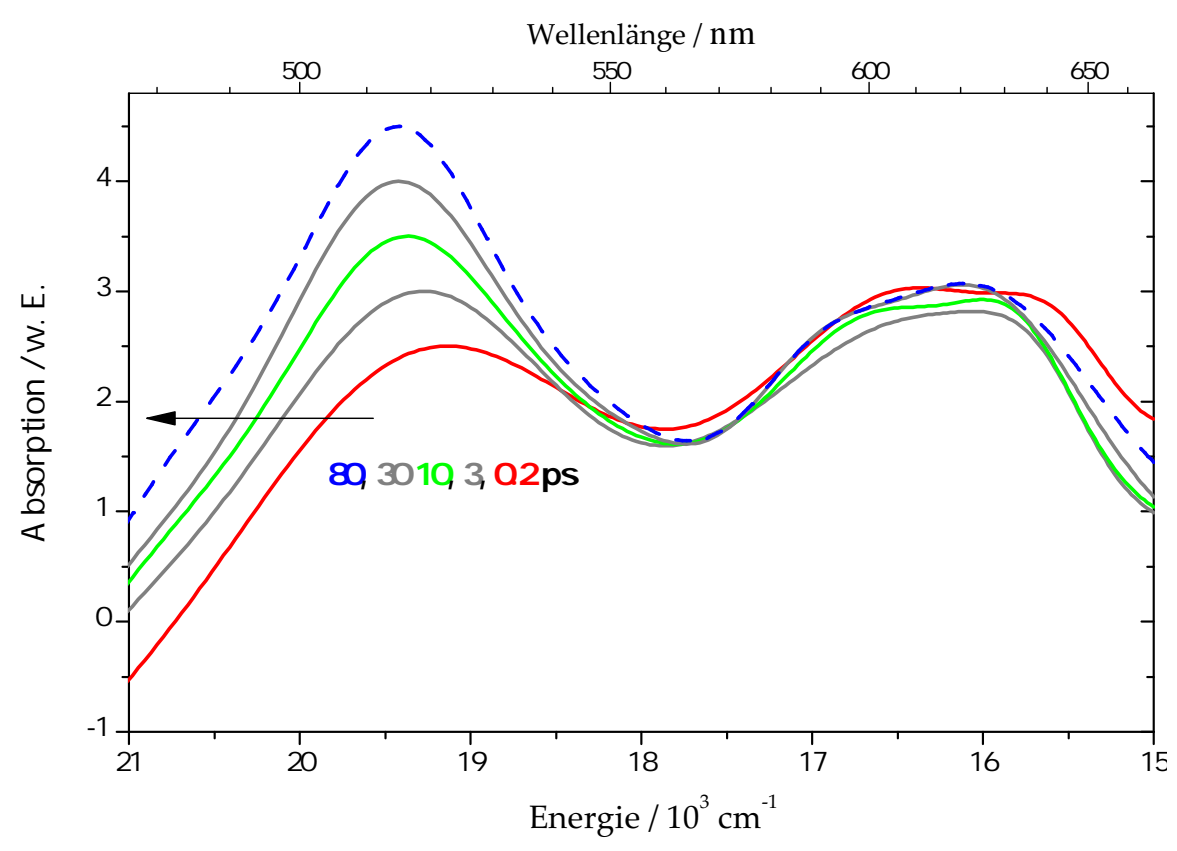

Abbildung 4.9: Ausschnitt der ESA-Banden von $12^{\prime} \mathrm{CA}$ in $\left[\mathrm{C}_{6} \mathrm{mim}\right]^{+}\left[\mathrm{Tf}_{2} \mathrm{~N}\right]^{-}$. 


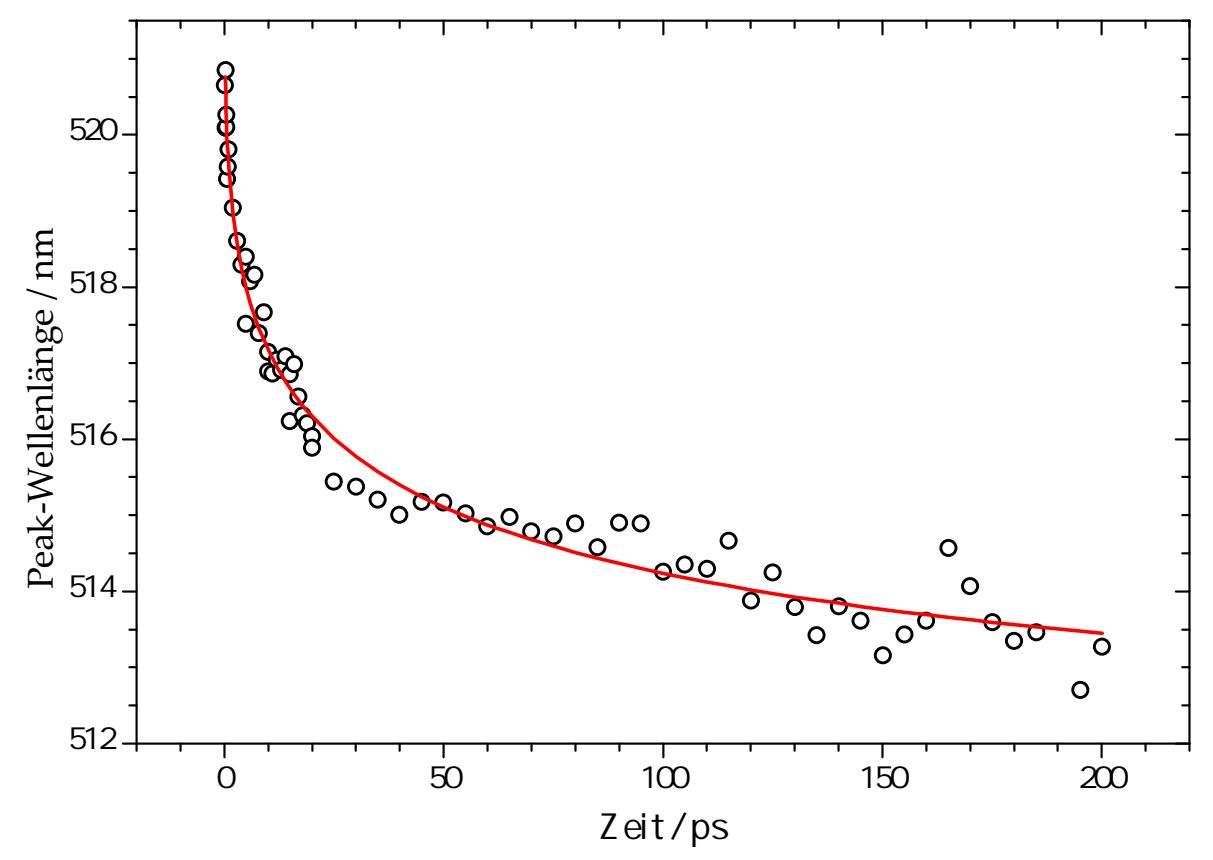

Abbildung 4.10: Entwicklung des Absorptionsmaximums der linken ESA-Bande von 12' $\mathrm{CA}$ in $\left[\mathrm{C}_{6} \mathrm{mim}\right]^{+}\left[\mathrm{Tf}_{2} \mathrm{~N}\right]^{-}$mit der Zeit.

Die PSCP-Spektren wurden mit einer globalen kinetischen Analyse ausgewertet. Hierbei wird der Übergang $S_{1} / I C T \rightarrow S_{0}$ in Betracht gezogen. Die Dynamik von 12 'CA in den ionischen Flüssigkeiten wird durch jeweils vier konsistente kinetische Spuren bei unterschiedlichen Wellenlängen in Abbildung 4.11- 4.14 beschrieben. Das langsame Abklingen der Kurven wird durch die Depopulation des $\mathrm{S}_{1}$ /ICT-Zustands hervorgerufen. Hierbei ergibt sich, wie oben besprochen, eine Abhängigkeit von der Art der ionischen Flüssigkeit. Die kinetischen Spuren werden wie in Abschnitt 4.2 gezeigt simuliert. Die transienten Spektren werden hinsichtlich ihrer spektralen Verschiebung in Abhängigkeit von der Zeit analysiert. Die linke ESA-Bande zeigt eine Blauverschiebung mit der Zeit. Diese Verschiebung ist ebenfalls abhängig von der Natur der ionischen Flüssigkeit. Die Ramanstruktur bei sehr frühen Zeiten während der Kreuzkorrelation wird allerdings bei der Analyse außen vor gelassen. Die erhaltenen Zeitkonstanten aus den kinetischen Spuren der transienten Spektren und für die Anpassung der Blauverschiebung der linken ESA-Bande sind in Tabelle 4.1 zusammengefasst. 


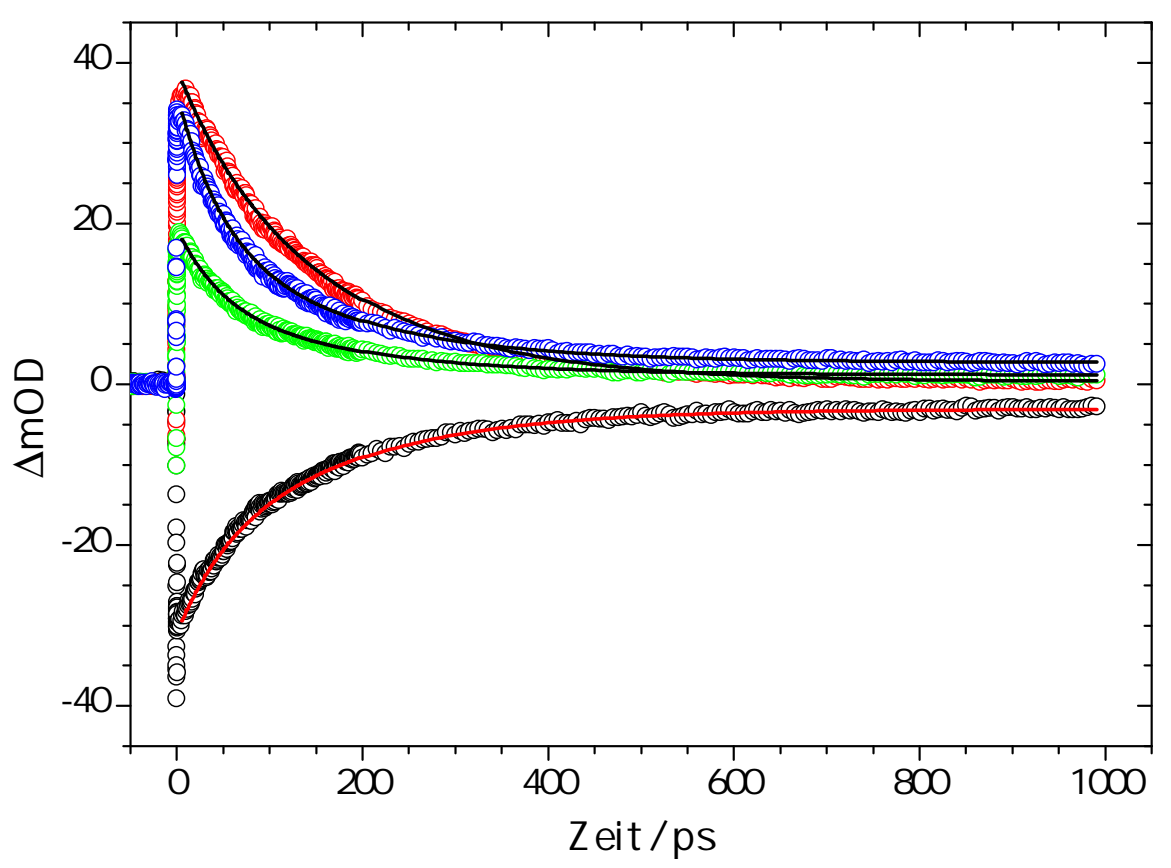

Abbildung 4.11: Kinetische Spuren mit globaler Anpassung von $12^{\prime} \mathrm{CA}$ in $\left[\mathrm{C}_{6} \mathrm{mim}^{+}\left[\mathrm{Tf}_{2} \mathrm{~N}\right]^{-}\right.$bei $423 \mathrm{~nm}(\circ), 514 \mathrm{~nm}(\circ), 564 \mathrm{~nm}(\circ)$ und $622 \mathrm{~nm}(\circ)$.

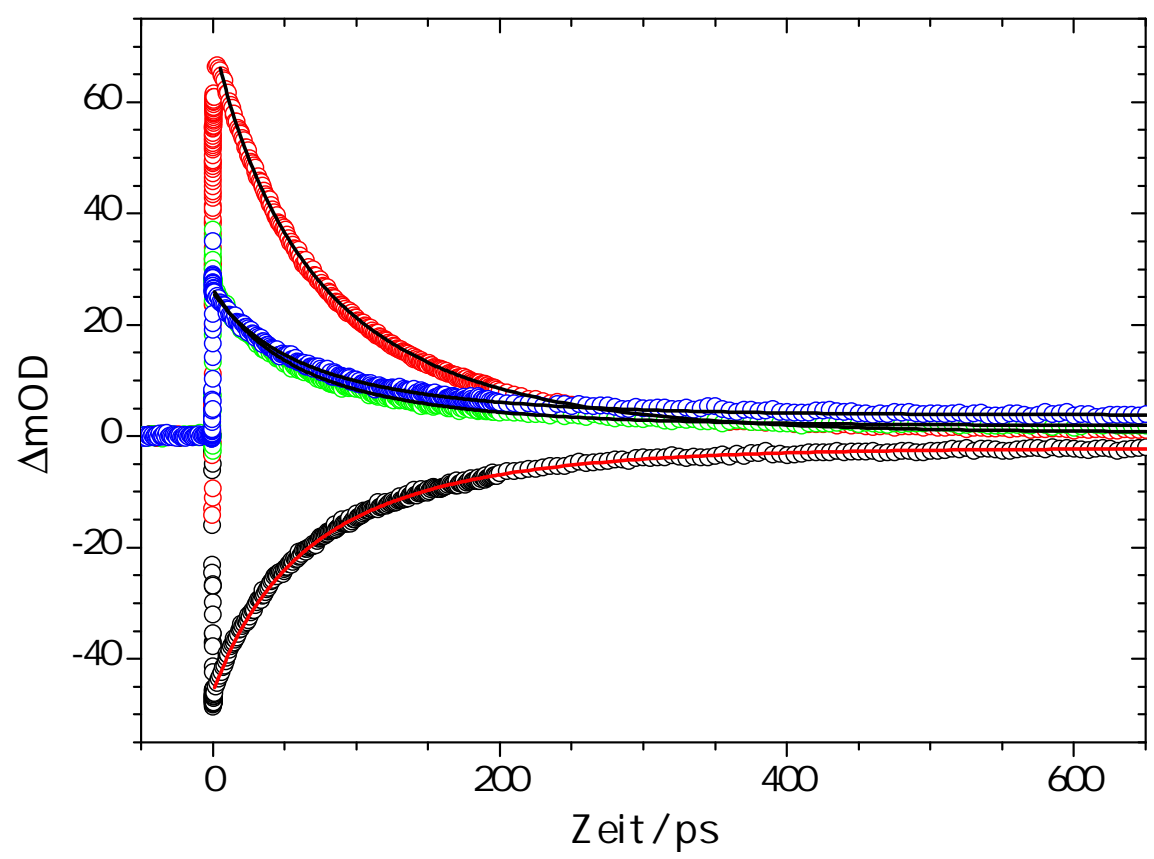

Abbildung 4.12: Wie Abbildung 4.11, aber in $\left[\mathrm{C}_{2} \mathrm{mim}\right]^{+}\left[\mathrm{EtSO}_{4}\right]^{-}$bei $431 \mathrm{~nm}(\circ), 531 \mathrm{~nm}(\circ), 597 \mathrm{~nm}$ (०) und $647 \mathrm{~nm}(\circ)$. 


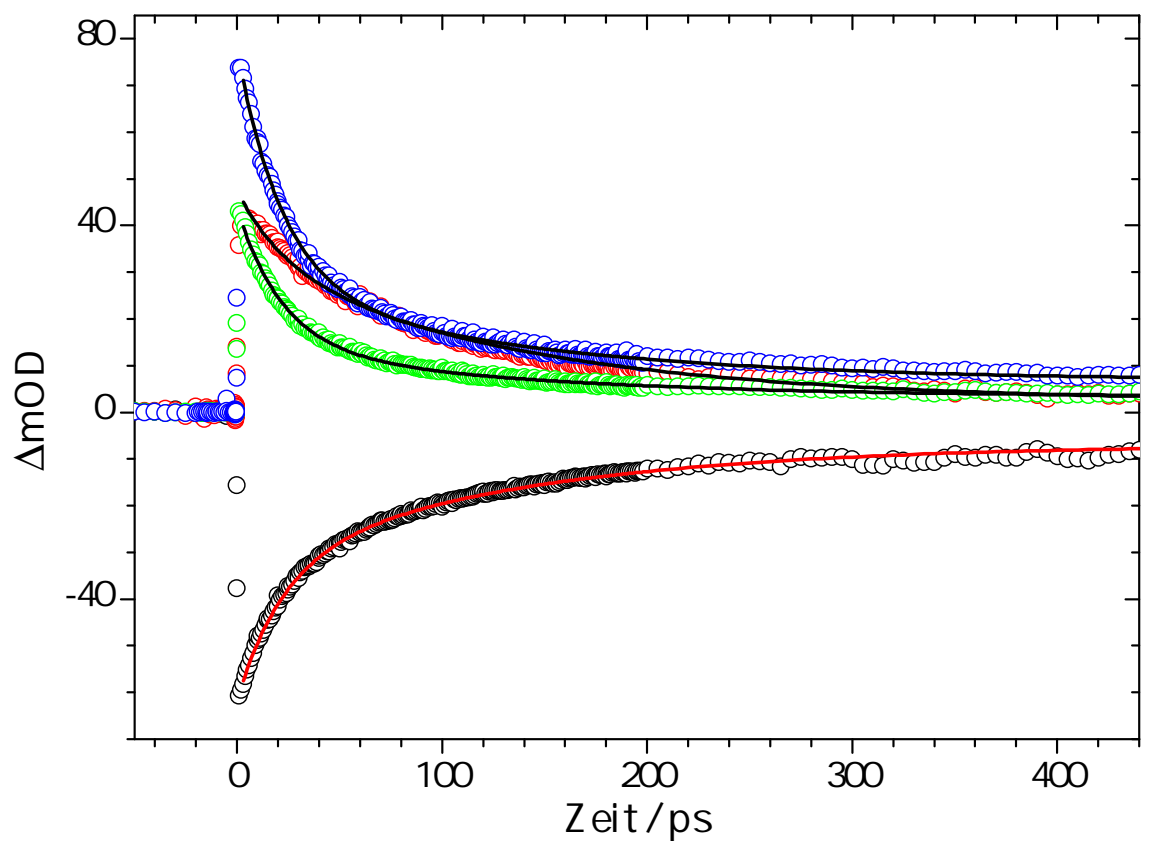

Abbildung 4.13: Wie Abbildung 4.11, aber in $\left[\mathrm{C}_{4} \mathrm{mim}\right]^{+}\left[\mathrm{Tf}_{2} \mathrm{~N}\right]^{-}$bei $422 \mathrm{~nm}(\circ), 505 \mathrm{~nm}(\circ), 555 \mathrm{~nm}$ (०) und $597 \mathrm{~nm}(\circ)$.

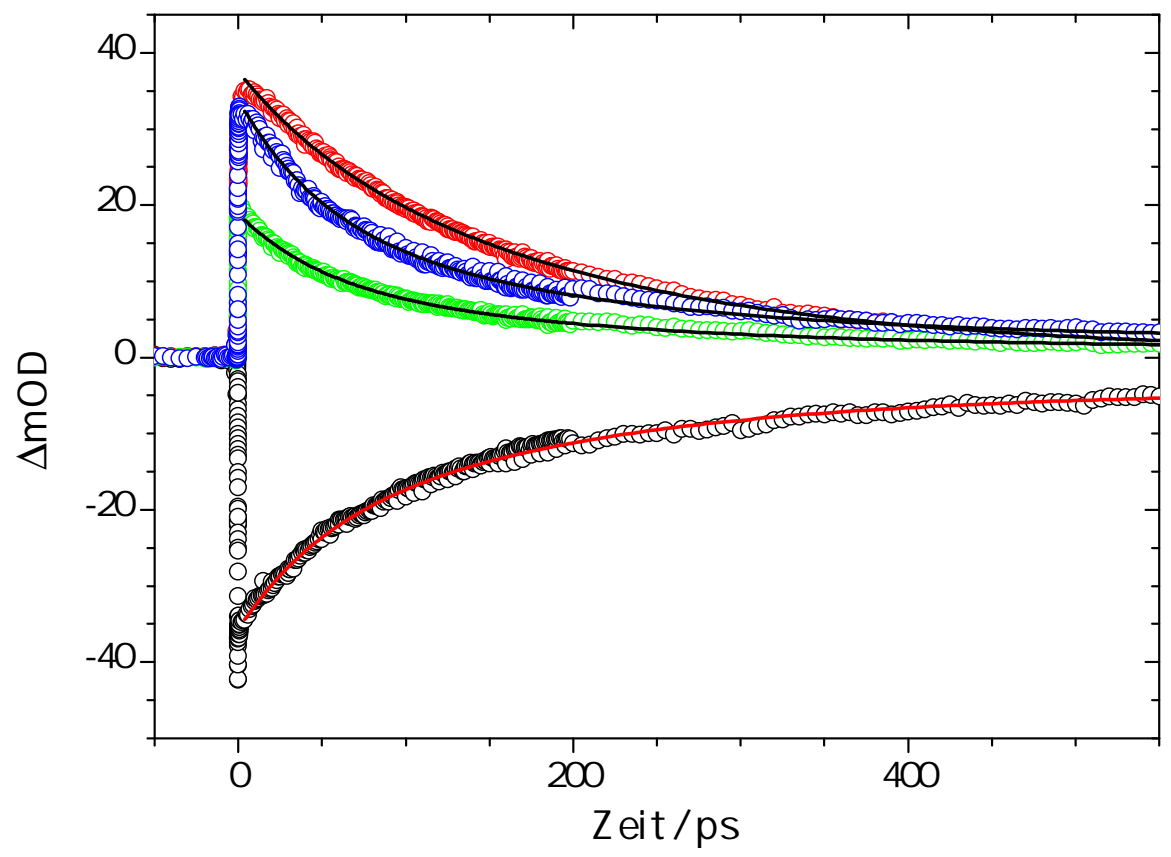

Abbildung 4.14: Wie Abbildung 4.11 aber in $\left[\mathrm{C}_{4} \mathrm{mmim}\right]^{+}\left[\mathrm{Tf}_{2} \mathrm{~N}\right]^{-}$bei $423 \mathrm{~nm}(\circ), 514 \mathrm{~nm}(\circ), 564$ $\mathrm{nm}(\circ)$ und $613 \mathrm{~nm}(\circ)$. 


\subsection{Experimentelle Ergebnisse für 12'-Apo- $\beta$-carotin-12'-al}

In diesem Kapitel werden die Ergebnisse der Pump-Supercontinuum-Probe (PSCP)-Messungen für 12'-Apo- $\beta$-carotin-12'-al (12'TAC) gezeigt. Die chemische Struktur dieser Sonde ist in Abbildung 4.1 aufgeführt. 12'TAC eignet sich ebenfalls zur Charakterisierung von Solvatationsprozessen und der Polarität von ionischen Flüssigkeiten (ILs). Die Lebensdauer des ersten angeregten Zustands ( $\left.\mathrm{S}_{1} / \mathrm{ICT}\right)$ zeigt wie bei anderen Carbonylcarotinoiden eine Abhängigkeit von der Polarität des Lösungsmittels. Die Polaritätsskala für organische Lösungsmittel ist im Fall dieser aldehydsubstituierten Sonde etwas weiter gefasst als bei 12'Apo- $\beta$-carotin-12'-säure und reicht von 220 ps in $n$-Hexan bis 8 ps in Methanol. ${ }^{79}$ Hierbei handelt es sich um Zeitkonstanten für die schnelle innere Konversion von 12'TAC in den entsprechenden organischen Lösungsmitteln. Diese wurden mittels transienter Absorptionsspektroskopie (TA) ermittelt. Es gibt bereits erste Messungen von 12'TAC in ionischen Flüssigkeiten mit dieser Methode. ${ }^{80}$ Anhand der Lebensdauer des $\mathrm{S}_{1}$ /ICT-Zustands könnte man die Polarität der gemessenen imidazoliumbasierten ILs zwischen Ethanol und Methanol einordnen. Dieser Befund ist konsistent mit den Messungen von 12'-Apo- $\beta$-carotin12'-säure in ILs. In dieser Arbeit konnte zum ersten mal die PSCP-Methode für 12'TAC angewandt werden. Dies gibt neue Kenntnisse auf dem Gebiet der mikroskopischen Wechselwirkung von molekularen Sonden in ILs.

Zur Charakterisierung der Solvatationsprozesse in ILs sind zwei verschiedene PSCPSpektren von 12'TAC in Abbildung 4.15 und 4.16 dargestellt. Es handelt sich hierbei um Messungen in $\left[\mathrm{C}_{4} \mathrm{mim}\right]^{+}\left[\mathrm{Tf}_{2} \mathrm{~N}\right]^{-}$und $\left[\mathrm{C}_{4} \mathrm{mmim}\right]^{+}\left[\mathrm{Tf}_{2} \mathrm{~N}\right]^{-}$. Die gleichen ionischen Flüssigkeiten sind ebenfalls mit der molekularen Sonde 12'CA untersucht worden, um einen Ver-

gleich der beiden Sonden herstellen zu können. Die Messungen bei frühen Zeiten sind im Fall von 12'TAC etwas deutlicher. Hier wird ebenfalls ein negativer Ground-State Bleach (GSB) der $\mathrm{S}_{0} \rightarrow \mathrm{S}_{2}$-Anregung und eine stimulierte Emission (SE) aus dem $\mathrm{S}_{2}$-Zustand beobachtet. Zur gleichen Zeit erscheint auch hier zwischen 500 und $770 \mathrm{~nm}$ eine Excited-State Absorption (ESA) aus dem $\mathrm{S}_{2}$-Zustand in höhere elektronische Zustände.

Im mittleren Teil der Abbildungen 4.15 und 4.16 wird die schnelle innere Konversion aus dem $S_{2}$ - in den $S_{1} /$ ICT-Zustand deutlich. Hier beobachtet man das Verschwinden der SE und ESA des $\mathrm{S}_{2}$-Zustands und den Aufbau der charakteristischen ESA-Banden des $\mathrm{S}_{1} /$ ICTZustands mit den beiden Peaks bei etwa 610 und 530 nm. Der Grund für das Auftreten 
mehrerer Banden ist die Absorption in verschiedene höher gelegene elektronische Zustände. Bei $650 \mathrm{~nm}$ ist kein klarer isosbestischer Punkt zu verzeichnen, was dafür spricht, dass eine zusätzliche Dynamik auf den Spektren liegt. Dies ist ein klares Zeichen für die Solvatation in ionischen Flüssigkeiten.

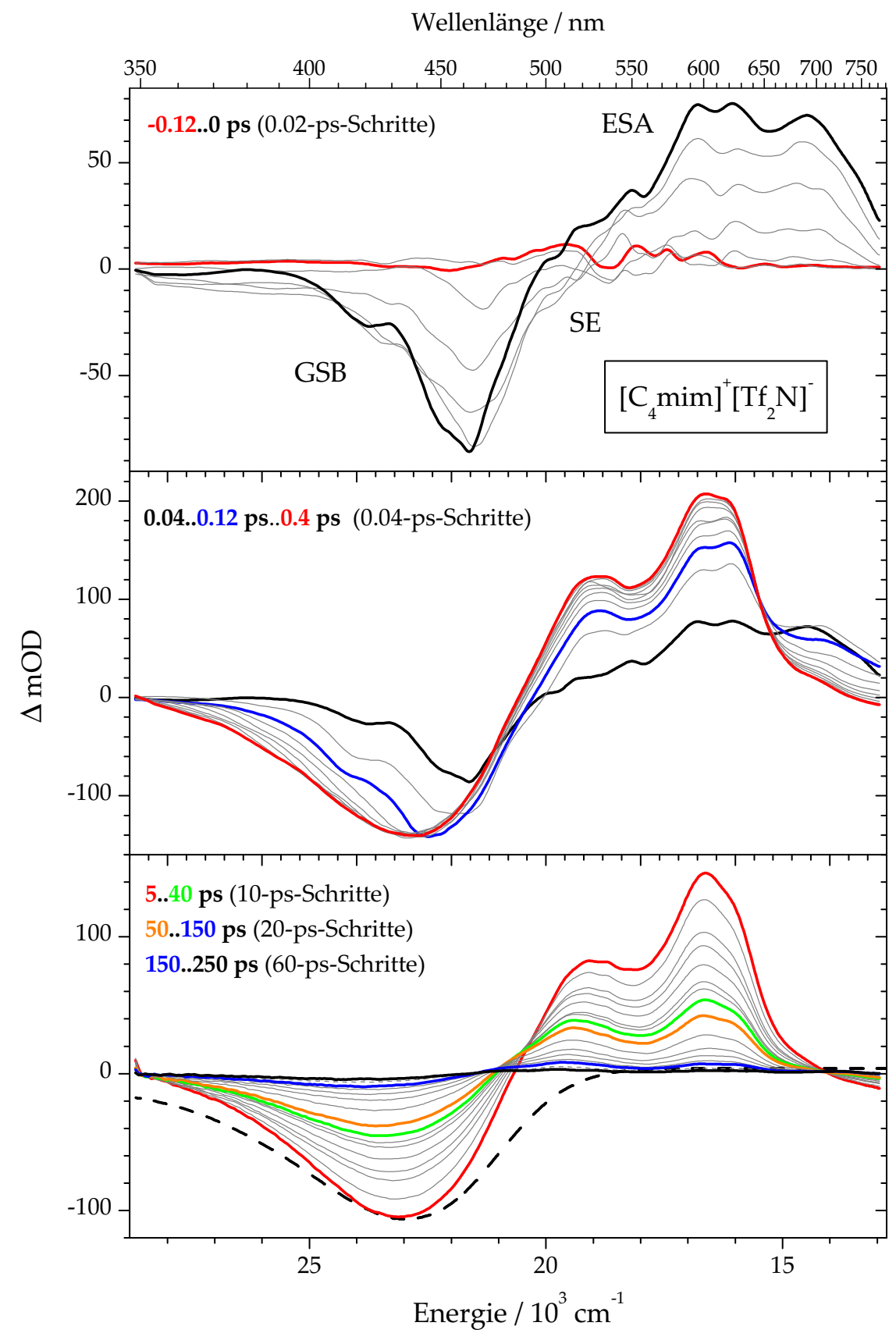

Abbildung 4.15: Transientes PSCP-Absorptionsspektrum von $12^{\prime} \mathrm{TAC}$ in $\left[\mathrm{C}_{4} \mathrm{mim}\right]^{+}\left[\mathrm{Tf}_{2} \mathrm{~N}\right]^{-}$bei einer Anregungswellenlänge von $480 \mathrm{~nm}$. $\Delta$ mOD ist die Änderung der optischen Dichte in $10^{-3}$. 


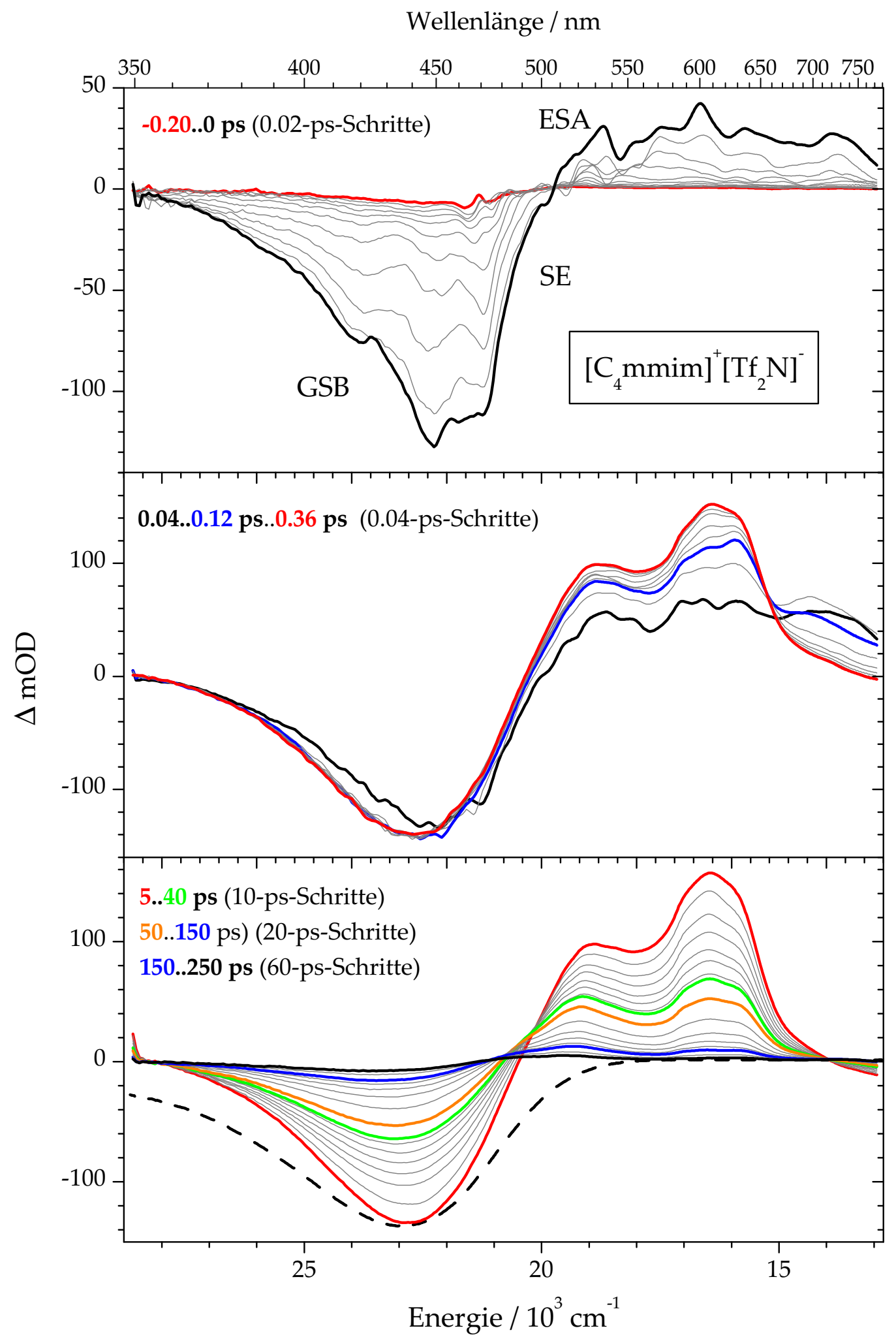

Abbildung 4.16: Wie Abbildung 4.15, aber in $\left[\mathrm{C}_{4} \mathrm{mmim}\right]^{+}\left[\mathrm{Tf}_{2} \mathrm{~N}\right]^{-}$. 
Auf einer längeren Zeitskala (unteres Feld) zeigt sich die Depopulation des $\mathrm{S}_{1} /$ ICTZustands. Der Grundzustand wird zeitgleich wieder aufgefüllt, was in einer Abnahme des GSB resultiert. Auffällig beim Verlauf der transienten Spektren ist erneut eine stetige Blauverschiebung. Dies ist besonders deutlich bei der linken Bande der ESA also im Bereich von 480 bis $550 \mathrm{~nm}$ zu sehen. Daher ist diese nochmals vergrößert in Abbildung 4.17 gezeigt. Der GSB zeigt ebenfalls diese Blauverschiebung mit der Zeit, hier liegt wahrscheinlich ein Teil einer ESA-Bande über dem Spektrum. Die spektrale Verschiebung wird durch die Solvatationsdynamik von 12'TAC in den ionischen Flüssigkeiten hervorgerufen. Ein weiterer Effekt, der in den ILs deutlich wird, ist das sich ändernde Verhältnis der beiden ESA-Banden mit der Zeit. Eine Zusammenfassung der globalen Analyse ist analog zu 12'CA im vorherigen Abschnitt in Tabelle 4.1 gegeben.

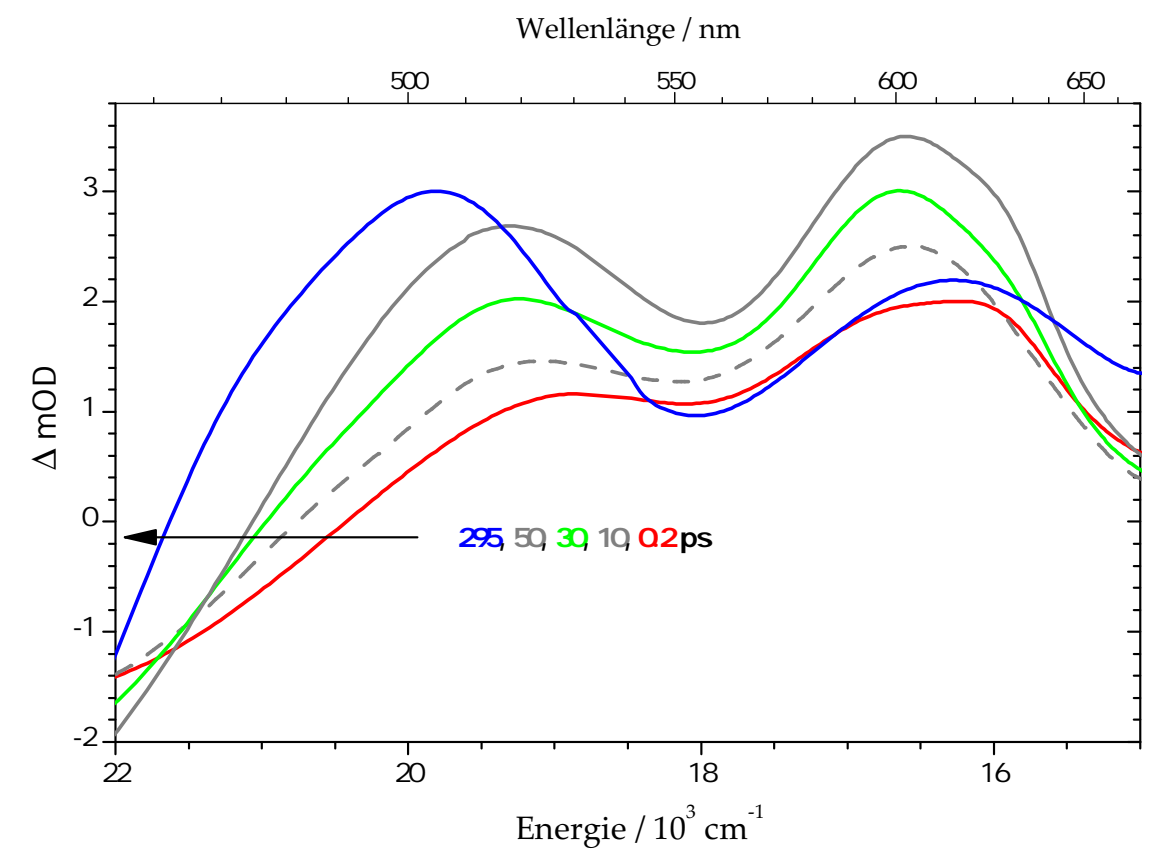

Abbildung 4.17: Ausschnitt der ESA-Banden von 12 TAC in $\left[\mathrm{C}_{4} \mathrm{mim}\right]^{+}\left[\mathrm{Tf}_{2} \mathrm{~N}\right]^{-}$.

Die Verschiebung des Absorptionsmaximums der linken ESA-Bande wird wie in Abschnitt 4.3 beschrieben ausgewertet. Ein Beispiel für die Abhängigkeit des Absorptionsmaximums von der Zeit ist in Abbildung 4.18 gegeben. Die Solvatationsdynamik wird dabei ab einer Zeit von 500 fs betrachtet. Bei früheren Zeiten ist die Charakterisierung der Solvatation durch die Überlagerung von spektralen Eigenschaften des $\mathrm{S}_{2}$-Zustands schwierig. 


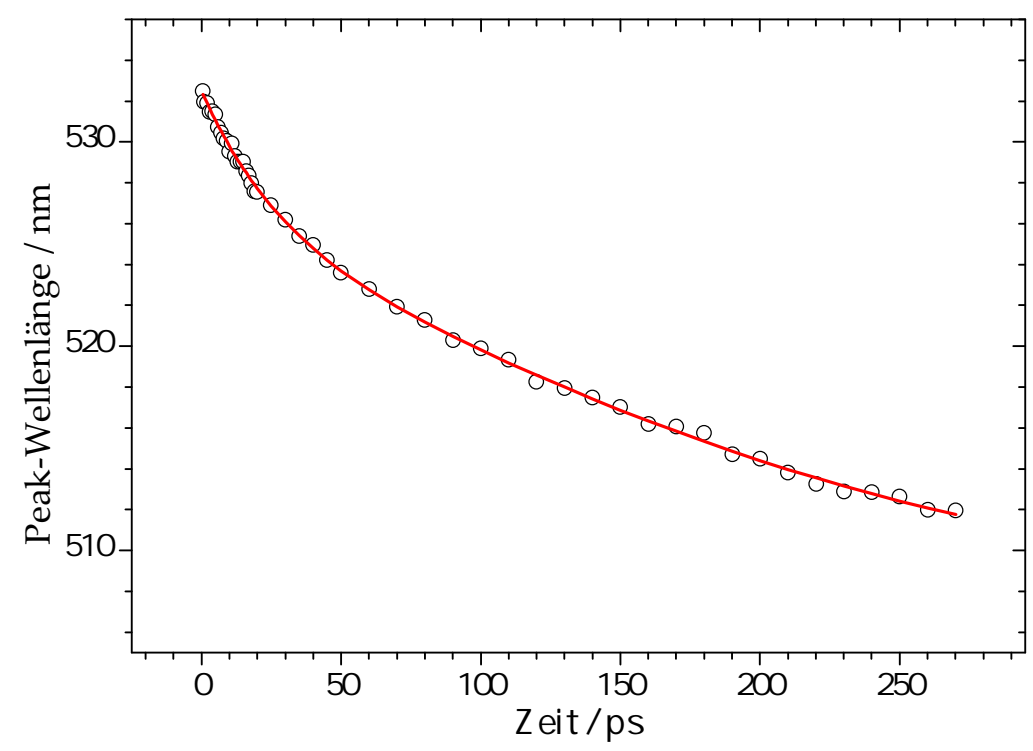

Abbildung 4.18: Entwicklung des Absorptionsmaximums der linken ESA-Bande von 12'TAC in $\left[\mathrm{C}_{4} \mathrm{mmim}\right]^{+}\left[\mathrm{Tf}_{2} \mathrm{~N}\right]^{-}$mit der Zeit.

Die Kinetik der transienten Spektren von 12 'TAC in $\left[\mathrm{C}_{4} \mathrm{mim}\right]^{+}\left[\mathrm{Tf}_{2} \mathrm{~N}\right]^{-}$und $\left[\mathrm{C}_{4} \mathrm{mmim}\right]^{+}$ $\left[\mathrm{Tf}_{2} \mathrm{~N}\right]^{-}$wird global ausgewertet. Vier repräsentative Anpassungen sind in den Abbildungen 4.19 und 4.20 gezeigt. Anschließend folgt in Tabelle 4.1 eine Übersicht aller ermittelten Zeitkonstanten von $12^{\prime}$ TAC und $12^{\prime} \mathrm{CA}$ in den verschiedenen ionischen Flüssigkeiten.

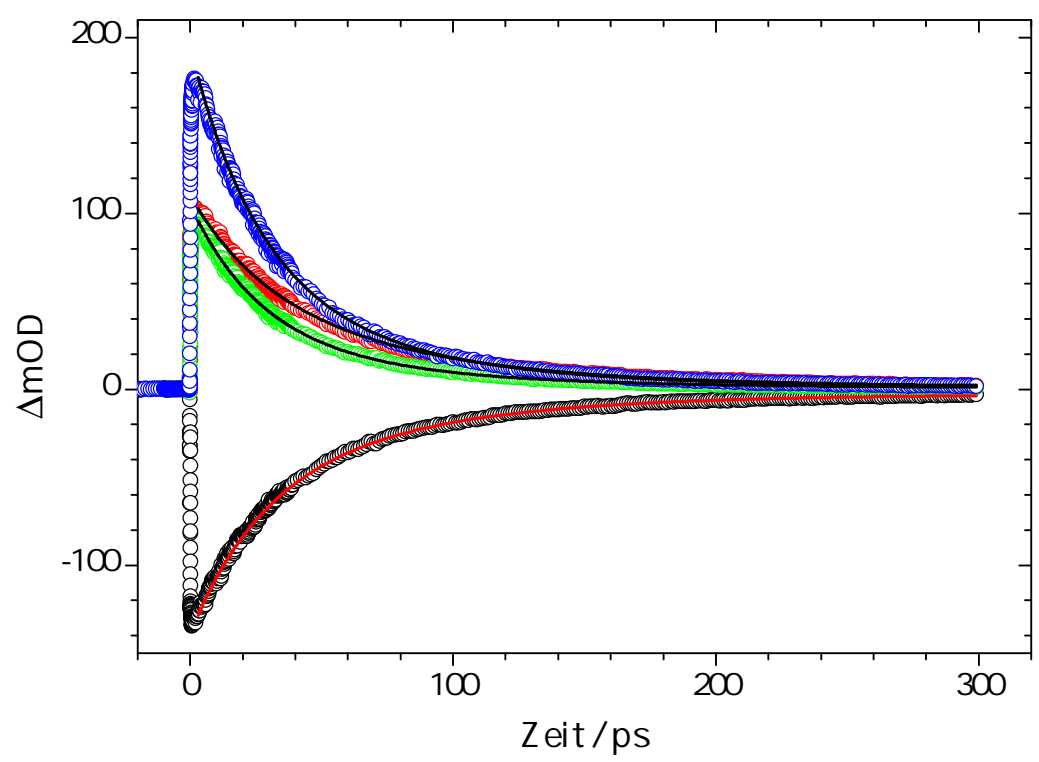

Abbildung 4.19: Kinetische Spuren mit globaler Anpassung von $12^{\prime} \mathrm{TAC}$ in $\left[\mathrm{C}_{4} \mathrm{mim}^{+}\left[\mathrm{Tf}_{2} \mathrm{~N}\right]^{-}\right.$bei $430 \mathrm{~nm}(\circ), 522 \mathrm{~nm}(\circ), 555 \mathrm{~nm}(\circ)$ und $605 \mathrm{~nm}(\circ)$. 


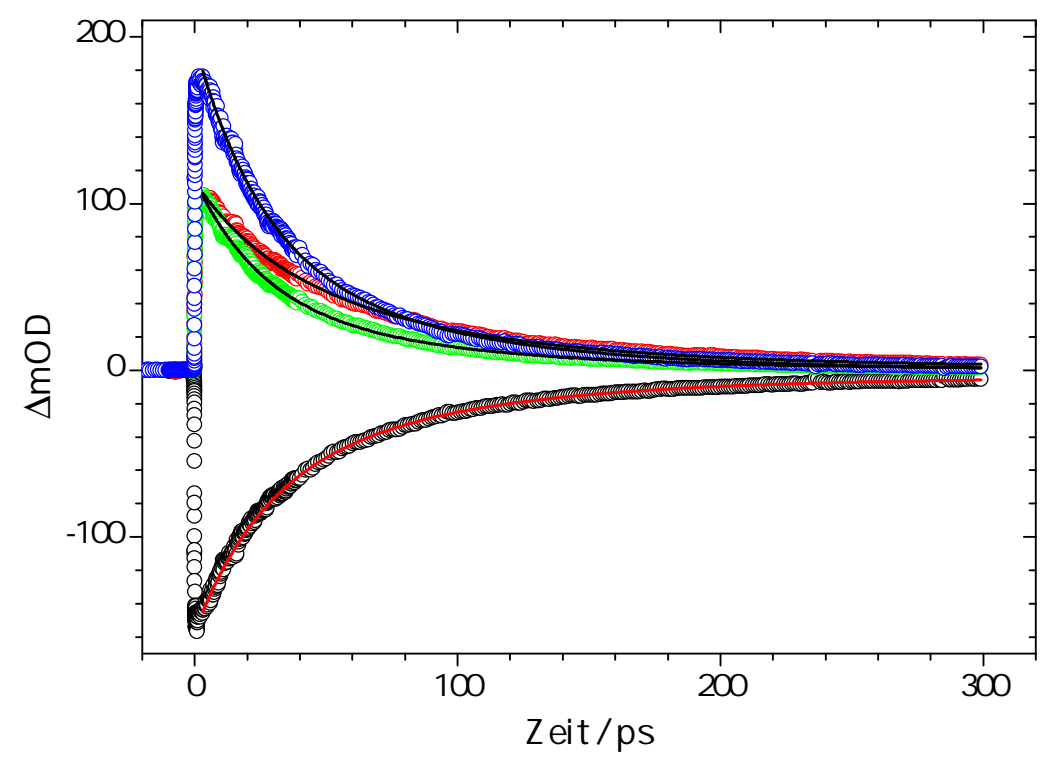

Abbildung 4.20: Kinetische Spuren mit globaler Anpassung von 12 'TAC in $\left[\mathrm{C}_{4} \mathrm{mmim}\right]^{+}\left[\mathrm{Tf}_{2} \mathrm{~N}\right]^{-}$bei $440 \mathrm{~nm}(\circ), 523 \mathrm{~nm}(\circ), 556 \mathrm{~nm}(\circ)$ und $606 \mathrm{~nm}(\circ)$.

Tabelle 4.1: Anpassungsparameter

\begin{tabular}{lccccc}
\hline $\mathrm{IL}$ & $\tau_{1, A}$ [ps] & $\tau_{1, B}$ [ps] & $\tau_{l}$ [ps] & $\tau_{s}$ [ps] & $\beta$ \\
\hline 12'-Apo- $\beta$-carotin-12'-säure $^{\prime}$ & & & & & \\
{$\left[\mathrm{C}_{6} \text { mim }\right]^{+}\left[\mathrm{Tf}_{2} \mathrm{~N}\right]^{-}$} & 158 & 45 & 32 & 0.1 & 0.33 \\
{$\left[\mathrm{C}_{2} \mathrm{mim}\right]^{+}\left[\mathrm{EtSO}_{4}\right]^{-}$} & 114 & 39 & 155 & 0.1 & 0.86 \\
{$\left[\mathrm{C}_{4} \mathrm{mim}\right]^{+}\left[\mathrm{Tf}_{2} \mathrm{~N}\right]^{-}$} & 132 & 22 & 7 & 0.1 & 0.30 \\
{$\left[\mathrm{C}_{4} \mathrm{mmim}^{+}\left[\mathrm{Tf}_{2} \mathrm{~N}\right]^{-}\right.$} & 184 & 49 & 7 & 0.2 & 0.30 \\
$\mathbf{1 2}^{\prime}$-Apo-$\beta$-carotin-12'-al & & & & & \\
{$\left[\mathrm{C}_{4} \text { mim }\right]^{+}\left[\mathrm{Tf}_{2} \mathrm{~N}\right]^{-}$} & 68 & 26 & 67 & 0.01 & 0.97 \\
{$\left[\mathrm{C}_{4} \mathrm{mmim}\right]^{+}\left[\mathrm{Tf}_{2} \mathrm{~N}\right]^{-}$} & 76 & 25 & 214 & 30 & 1.6 \\
\hline
\end{tabular}




\subsection{Diskussion}

Wie in der Einleitung erwähnt, hat die Polarität von Lösungsmitteln einen großen Einfluss auf chemische Reaktionen. Daher ist es wichtig, diese zu charakterisieren um letztendlich die Kinetik der Reaktionen besser verstehen zu können. Der Ansatz dieser Arbeit ist es, den Einfluss von ionischen Flüssigkeiten (ILs) auf die ultraschnelle Dynamik von molekularen Sonden zu studieren. Dazu sind diese Sonden mit transienter Absorptionsmethoden untersucht worden. Die in Kapitel 4.3 und 4.4 vorgestellten Ergebnisse werden im Folgenden diskutiert.

Die PSCP-Spektren werden im Hinblick auf die Relaxation des $\mathrm{S}_{1}$ / ICT-Zustands analysiert und die erhaltenen Zeitkonstanten sind in Tabelle 4.1 zu finden. Die PSCP-Spektren beider Apocarotinoide zeigen in den ionischen Flüssigkeiten eine Blauverschiebung der ESA-Bande des $\mathrm{S}_{1} /$ ICT-Zustands. Dieses Verhalten ist in dem polaren Lösungsmittel Methanol ebenfalls zu verzeichnen, wohingegen in Tetrahydrofuran eine solch klare Verschiebung nicht beobachtet wird. ${ }^{82}$ Die spektrale Entwicklung in Methanol findet auf einer anderen Zeitskala als in den ILs statt. Diese Dynamik, die in allen Lösungsmitteln beobachtet wird, ist typisch für die Relaxation des Lösungsmittels um einen dipolaren ChargeTransfer-Zustand. $\frac{110}{10}$ Die Blauverschiebung ist nicht für alle Übergänge gleich. Wie oben besprochen ist, gibt es eine Verschiebung der linken ESA-Bande bei etwa 480 bis $550 \mathrm{~nm}$ und der GSB scheint ebenfalls ins Blaue verschoben zu werden, wobei es sich hier um eine weitere ESA-Bande handeln muss, die über dem Signal liegt. Bei der rechten ESA-Bande ist diese Verschiebung nicht so stark ausgeprägt. Dies kann durch den unterschiedlichen elektronischen Charakter der angeregten Zustände erklärt werden. Die beiden Zustände, die für eine spektrale Verschiebung verantwortlich sind, sollten ein kleineres Dipolmoment haben als der $\mathrm{S}_{1} / \mathrm{ICT}$-Zustand. Der obere Zustand, der für die rechte Bande des ESA-Spektrums verantwortlich ist, hat wahrscheinlich ein ähnliches Dipolmoment, daher kommt es hier nur zu einer sehr schwachen spektralen Verschiebung mit der Solvensrelaxation. Die Amplituden der beiden sichtbaren ESA-Banden gleichen sich im Verlauf der Relaxation an. Dies könnte durch die Änderung der Franck-Condon-Faktoren und der Umverteilung der Population auf der Potentialfläche des $\mathrm{S}_{1} /$ ICT-Zustands während der Relaxation hervorgerufen werden.

Die spektrale Entwicklung der molekularen Sonden in den ionischen Flüssigkeiten wird 
am besten durch eine ultraschnelle Subpikosekunden- und eine langsamere Komponente im Pikosekundenregime beschrieben. Dies ist typisch für Relaxationsprozesse bei denen die Bewegung der Lösungsmittelmoleküle, oder im Fall von ILs der Ionen, eine Rolle spielt. ${ }^{111}$ Über die funktionale Form zur Anpassung der spektralen Entwicklung lässt sich natürlich streiten. Wie von ARZHANTSEV vorgeschlagen,,$\frac{76}{1}$ lassen sich auch die Daten dieser Arbeit am besten mit einer Summe aus einer monoexponetiellen und einer gestrecktexponentiellen Funktion anpassen (siehe Gleichung 4.2). Durch eine globale Analyse der Spektren lässt sich das Maximum der linken ESA-Bande als Funktion der Zeit ermitteln. Aus diesem Verlauf resultieren die beiden Zeitkonstanten, die charakteristisch sind für die Solvensrelaxation der spezifischen ionischen Flüssigkeiten.

Wenn man die ionische Flüssigkeit $\left[\mathrm{C}_{4} \mathrm{mim}\right]^{+}\left[\mathrm{Tf}_{2} \mathrm{~N}\right]^{-}$mit der methylierten Spezies $\left[\mathrm{C}_{4^{-}}\right.$ mmim $]^{+}\left[\mathrm{Tf}_{2} \mathrm{~N}\right]^{-}$vergleicht, fällt auf, dass die Werte für die spektrale Entwicklung beider molekularen Sonden im Fall von $\left[\mathrm{C}_{4} \mathrm{mmim}\right]^{+}\left[\mathrm{Tf}_{2} \mathrm{~N}\right]^{-}$größer sind. Nach KOBRAK wird die kürzere Komponente $\left(\tau_{s}\right)$ der Relaxation durch die Bewegung der Ionen in unmittelbarer Nachbarschaft zur Sonde verursacht. Die langsame Komponente $\left(\tau_{l}\right)$ ist langweitreichenderen Wechselwirkungen zuzuordnen. ${ }^{112}$ Folgt man der Interpretation seiner MDSimulationen von Coumarin-153 in 1-Butyl-2-methylimidazoliumhexafluorophosphat dann lässt sich der Befund für die beiden imidazoliumbasierten ionischen Flüssigkeiten mit leicht konstitutionellem Unterschied wie folgt erklären. Die Translation der Ionen zu Beginn der Solvatation ist durch eine leicht erhöhte sterische Behinderung durch die zusätzliche Methylgruppe gehindert. Daher sind die $\tau_{s}$-Werte für $\left[\mathrm{C}_{4} \mathrm{mmim}\right]^{+}\left[\mathrm{Tf}_{2} \mathrm{~N}\right]^{-}$etwas höher. Bei der langsameren Komponente ist der Unterschied nicht ganz so groß. Die $\tau_{l}$ Werte werden wahrscheinlich durch Käfigdeformationen und Reorientierungsprozesse der ionischen Flüssigkeiten beeinflusst. $\frac{31113}{}$ Diese Prozesse scheinen in der methylierten Spezies etwas langsamer zu sein, auch hier kann es zu einer leicht erhöhten sterischen Hinderung kommen. Aufgrund der geringeren Qualität der Messungen von $12^{\prime} \mathrm{CA}$ gerade in $\left[\mathrm{C}_{4} \mathrm{mim}\right]^{+}$$\left[\mathrm{Tf}_{2} \mathrm{~N}\right]^{-}$sind hier die ermittelten Werte nicht so genau. In $\left[\mathrm{C}_{4} \mathrm{mim}\right]^{+}\left[\mathrm{Tf}_{2} \mathrm{~N}\right]^{-}$und $\left[\mathrm{C}_{4} \mathrm{~m}-\right.$ mim $]^{+}\left[\mathrm{Tf}_{2} \mathrm{~N}\right]^{-}$ergeben sich für die spektrale Verschiebung gleiche Zeitkonstanten. Nach den Ergebnissen von 12'TAC kann aber vermutet werden, dass es auch hier eigentlich Unterschiede geben müsste.

Messungen von reinen imidazoliumbasierten ILs ohne Zuhilfenahme von molekularen 
Sonden können mit den Methoden der optischen Kerr-Effekt Spektroskopie (OKE), dielektrischen Relaxationsspektroskopie (DRS) und Terahertz- und FTIR-Spektroskopie im Bereich von $200 \mathrm{MHz}-10 \mathrm{THz}$ durchgeführt werden.114 [117 Die Spektren dieser Messungen sind relativ breit und beinhalten verschiedene sich überlappende Banden der Lösungsmittel. Es sind Zeitkonstanten für die Relaxation der ionischen Flüssigkeiten von 80 fs bis 5 ns gefunden worden. Dies ist konsistent mit den ultraschnellen spektroskopischen Methoden dieser Arbeit. Allerdings konnte hier nur bis maximal 1.5 ns gemessen werden.

Mit der molekularen Sonde $12^{\prime} \mathrm{CA}$ sind drei ionische Flüssigkeiten mit gleichem Anion und imidazoliumbasierten Kationen mit unterschiedlicher Alkylkettenlänge gemessen worden. Anhand der kinetischen Spuren der PSCP-Messungen konnten zwei Zeitkonstanten $\tau_{1, A}$ und $\tau_{1, B}$ bestimmt werden. Die langsamere Zeitkonstante $\tau_{1, A}$ wird der Lebensdauer des $\mathrm{S}_{1} /$ ICT-Zustands zugeordnet. Diese Lebensdauern von 12'CA folgen dem Trend $\left[\mathrm{C}_{4} \mathrm{mmim}\right]^{+}\left[\mathrm{Tf}_{2} \mathrm{~N}\right]^{-}>\left[\mathrm{C}_{6} \mathrm{mim}^{+}\left[\mathrm{Tf}_{2} \mathrm{~N}\right]^{-}>\left[\mathrm{C}_{4} \mathrm{mim}^{+}\left[\mathrm{Tf}_{2} \mathrm{~N}\right]^{-}\right.\right.$. Die molekulare Sonde 12'TAC zeigt ebenfalls einen Unterschied zwischen $\left[\mathrm{C}_{4} \mathrm{mmim}\right]^{+}\left[\mathrm{Tf}_{2} \mathrm{~N}\right]^{-}$und $\left[\mathrm{C}_{4} \mathrm{mim}\right]^{+-}$ $\left[\mathrm{Tf}_{2} \mathrm{~N}\right]^{-}$, wobei die Lebensdauern generell kleiner sind als bei $12^{\prime} \mathrm{CA}$. Dieser Unterschied zwischen den beiden Sonden wird auch in polaren organischen Lösungsmitteln gefunden. ${ }^{81}$ Die Lebensdauern lassen sich gut mit dem Kationendurchmesser korrelieren. Dies geschieht zur Verdeutlichung in Abbildung 4.21. Die an C-2 Position methylierte ionische Flüssigkeit hat in diesem Vergleich die höchste Lebensdauer. Dies könnte daran liegen, dass eine mögliche Stabilisierung des $\mathrm{S}_{1}$ / ICT-Zustands durch Coulomb-Wechselwirkungen mit dem Kation schlechter möglich ist. Auch eine Stabilisierung durch Wasserstoffbrückenbindungen ist denkbar. Die beiden anderen ionischen Flüssigkeiten könnten über das H-Atom an C-2 Position Wasserstoffbrücken aufbauen.

In dieser und früheren Arbeiten konnte gezeigt werden, dass sich die Apocarotinoide 12 'CA und 12'TAC als molekulare Sonden für die ultraschnelle transiente Absorptionsspektroskopie zur Untersuchung der mikroskopischen Umgebung in Lösungsmitteln eignen. ${ }^{[3] 81+83}$ Durch die PSCP-Spektroskopie haben sich neue Einblicke in die Solvatationsdynamik von ionischen Flüssigkeiten ergeben. 
Ein anderer Ansatz zum Studium der ionischen Flüssigkeiten ist das Mischen mit organischen Lösungsmitteln, deren Eigenschaften bereits besser bekannt sind. Daraus könnten sich auch neue Erkenntnisse auf dem Gebiet der ionischen Flüssigkeiten ergeben. Dieser Ansatz wird nun im folgenden Kapitel aufgenommen.

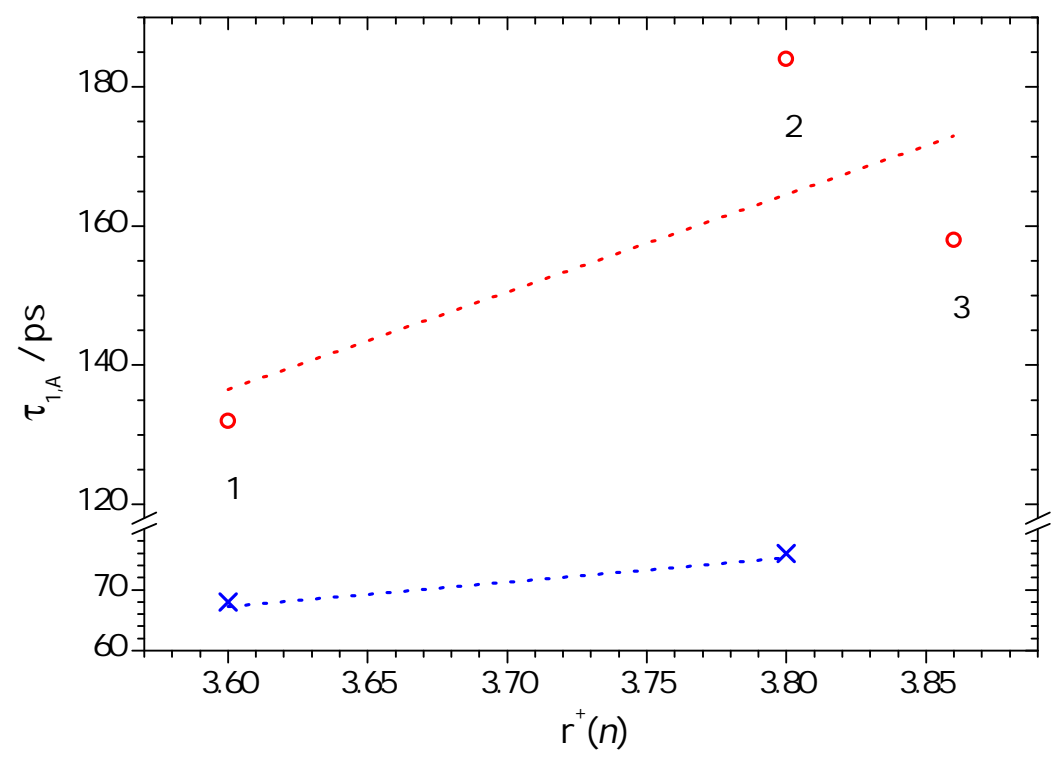

Abbildung 4.21: Lebensdauern $\tau_{1, A}$ der molekularen Sonden 12'CA (०) und 12'TAC $(\times)$ in den ionischen Flüssigkeiten $\left[\mathrm{C}_{4} \mathrm{mim}\right]^{+}\left[\mathrm{Tf}_{2} \mathrm{~N}\right]^{-}(1),\left[\mathrm{C}_{4} \mathrm{mmim}\right]^{+}\left[\mathrm{Tf}_{2} \mathrm{~N}\right]^{-}$(2) und $\left[\mathrm{C}_{6} \mathrm{mim}\right]^{+}\left[\mathrm{Tf}_{2} \mathrm{~N}\right]^{-}(3)$ in Abhängigkeit vom Kationenradius $\mathrm{r}^{+}$. 



\section{Kapitel 5}

\section{Mikroskopische Eigenschaften von ionischen Flüssigkeiten nach Zugabe von Acetonitril}

Ionische Flüssigkeiten (ILs) eignen sich hervorragend zur Manipulation physiko-chemischer Prozesse. Durch Variation von Kationen und Anionen lassen sich Lösungsmittel mit gewünschten Eigenschaften maßschneidern. Dies führt zu einer Vielzahl von Anwendungen, unter anderem in der Synthese. Ein weiterer Freiheitsgrad, um die Eigenschaften zu steuern, stellt das Mischen mit organischen Lösungsmitteln dar. ${ }^{2}$

Die Untersuchung der Solvatationsdynamik gelöster Moleküle in ILs ist für das Verständnis der chemischen Reaktivität fundamental. Das Auftreten mangelhafter Löslichkeit bestimmter Moleküle in ILs stellt ein erhebliches Problem für ihre Anwendbarkeit dar. Durch das Herstellen binärer Mischungen könnte dieses Problem umgangen werden, indem die Löslichkeit erhöht wird. Aufgrund der Zugabe von molekularen Lösungsmitteln wird die lokale Umgebung der gelösten Probemoleküle geändert, daher ist es interessant das Verhalten in binären Mischungen mit den Ergebnissen von Experimenten an reinen Komponenten zu vergleichen.

Dielektrische Spektren über einen weiten Frequenzbereich von 0.2 - $89 \mathrm{GHz}$ für das System 1-N-Ethyl-3-N-methylimidazoliumethylsulfat $\left(\left[\mathrm{C}_{2} \mathrm{mim}\right]^{+}\left[\mathrm{EtSO}_{4}\right]^{-}\right) /$Dichlormethan führten BUCHNER zu der Aussage, dass die ionische Flüssigkeit ihre Struktur bis zu einer hohen Verdünnung $\left(\mathrm{x}_{I L}=0.2\right)$ beibehält. ${ }^{118}$ Bei anderen Mischungen wurde qualitativ das gleiche Ergebnis gefunden. Die mikroskopische Umgebung eines Probemoleküls in einer binären Mischung könnte also Komponenten beider Spezies enthalten. Ein weiteres denkbares Szenario wäre eine präferierte Solvatation durch nur eines der gemischten Lö- 
sungsmittel, wie es z. B. in einigen so genannten Gas-Expanded Liquids (GXLs) der Fall ist, die von MARONCELLI untersucht worden sind. $119-121$ Dies sind Mischungen aus organischen Lösungsmitteln und Gasen, wie z. B. $\mathrm{CO}_{2}$, nahe am kritischen Punkt. Es konnte eine bevorzugte Löslichkeit einiger Probemoleküle in der flüssigen Komponente nachgewiesen werden. Dieses Verhalten könnte auch auf binäre Mischungen ionischer Flüssigkeiten mit organischen Lösungsmitteln zutreffen.

In der vorliegenden Arbeit sind binäre Mischungen von 1-N-Hexyl-3-N-methyl-imidazolium-bis(trifluoromethylsulfonyl)imid $\left(\left[\mathrm{C}_{6} \mathrm{mim}\right]^{+}\left[\mathrm{Tf}_{2} \mathrm{~N}\right]^{-}\right)$und 1-N-Ethyl-3-N-methylimidazoliumethylsulfat $\left(\left[\mathrm{C}_{2} \mathrm{mim}\right]^{+}\left[\mathrm{EtSO}_{4}\right]^{-}\right)$mit Acetonitril untersucht worden. Spektroskopische Messungen an 12'-Apo- $\beta$-carotin-12'-säure (12'CA) in den genannten Mischungen werden im besonderen Hinblick auf mögliche präferierte Solvatation durchgeführt. Die reinen Komponenten sind bereits mittels transienter Absorptionsspektroskopie untersucht worden. ${ }^{3381}$ Acetonitril lässt sich besonders gut mit den ionischen Flüssigkeiten mischen und die Lebensdauer $\tau_{1}$ des $\mathrm{S}_{1}$ /ICT-Zustands von 12 'CA ist im Vergleich mit den ILs relativ klein ist. Somit ergibt sich ein möglichst großes Fenster für die Messungen der Mischungen. Der Unterschied von $\tau_{1}$ der reinen Komponenten $\left[\mathrm{C}_{6} \mathrm{mim}\right]^{+}\left[\mathrm{Tf}_{2} \mathrm{~N}\right]^{-}$und Acetonitril beträgt $\Delta \tau_{1}=39 \mathrm{ps}$ und von $\left[\mathrm{C}_{2} \mathrm{mim}\right]^{+}\left[\mathrm{EtSO}_{4}\right]^{-}$im Vergleich mit Acetonitril $\Delta \tau_{1}$ $=32$ ps. Diese großen Differenzen ermöglichen eine genaue Analyse des gesamten Zusammensetzungsbereichs der binären Mischungen.

\subsection{Kinetisches Modell zur Interpretation der transienten Absoprtsionsmessungen}

Ausgehend von einem Drei-Zustände-Modell der Apocarotinoide (siehe Abbildung 4.2) werden in dem kinetischen Modell zwei Zeitkonstanten berücksichtigt. Die innere Konversion $\mathrm{S}_{2} \rightarrow \mathrm{S}_{1} / \mathrm{ICT}$ hat eine relativ kurze Lebensdauer $\tau_{2}$ von etwa $100 \mathrm{fs}$ und stellt die eine Zeitkonstante dar (Gleichung 5.1). Die Lebensdauer $\tau_{1}$ der inneren Konversion $\mathrm{S}_{1} / \mathrm{ICT} \rightarrow \mathrm{S}_{0}$ der betrachteten Apocarotinoide ist von der Polarität des Lösungsmittels abhängig. Die Änderung der Population dieses Zustandes beinhaltet die zweite erwähnte Zeitkonstante (Gleichung 5.2). Ein Beispiel eines typischen transienten Signals ist in Abbildung 5.1 zu finden. 


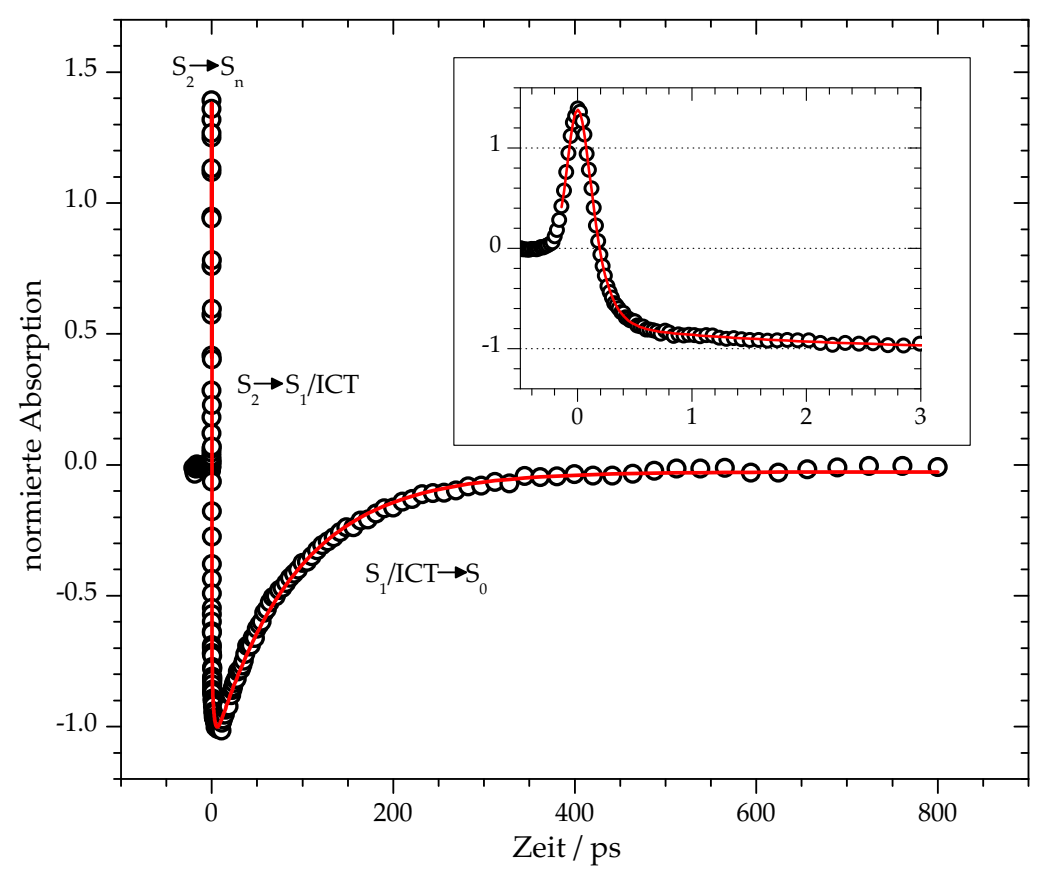

Abbildung 5.1: Typisches Signal einer TA-Messung (०) mit kinetischer Anpassung ( - ) von 12'Apo- $\beta$-carotin-12'-säure in $\left[\mathrm{C}_{6} \mathrm{mim}\right]^{+}\left[\mathrm{Tf}_{2} \mathrm{~N}\right]^{-}$.

Die Signale beginnen mit einer scharfen Spitze, die von der Absorption aus dem zweiten angeregten Zustand $S_{2}$ in ein höher gelegenes Niveau $S_{n}$ herrührt. Diesem transienten Absorptionssignal folgt ein Signal der stimulierten Emission (SE), welche dem niedrig liegenden elektronischen Zustand der Apocarotinoide mit intramolekularem ChargeTransfer (ICT)-Charakter, also dem Übergang $\mathrm{S}_{1} / \mathrm{ICT} \rightarrow \mathrm{S}_{0}$, zuzuordnen ist. Das kinetische Modell beinhaltet eine weitere Zeitkonstante bzw. Lebensdauer $\tau_{3}$. Diese Zeitkonstante lässt sich anhand des Drei-Zustände-Modells nicht erklären. Es handelt sich hierbei um Relaxationsprozesse des $\mathrm{S}_{1}$ /ICT-Zustands welche durch die Solvatationsdynamik in den ionischen Flüssigkeiten hervorgerufen werden.

Zur Aufstellung einer mathematischen Beschreibung des kinetischen Modells $S_{2} \rightarrow S_{1}$ $/ \mathrm{ICT} \rightarrow \mathrm{S}_{0}$ sei zunächst die Änderung der Population der drei beteiligten Zustände mit der Zeit betrachtet. Für den Pumppuls wird ein gaußförmiges Profil angenommen. Das Geschwindigkeitsgesetz für die Depopulation des $\mathrm{S}_{2}$-Zustands lautet dann:

$$
\frac{d\left[S_{2}\right]}{d t}=k_{A} \cdot \exp \left(-\frac{t^{2}}{\gamma^{2}}\right)-k_{2 \rightarrow 1} \cdot\left[S_{2}\right]
$$


Die Moleküle werden mit einer Geschwindigkeitskonstanten $k_{A}$ angeregt $\left(\mathrm{S}_{0} \rightarrow \mathrm{S}_{2}\right)$ und für die schnelle innere Konversion $S_{2} \rightarrow S_{1} /$ ICT steht die Zeitkonstante $k_{2 \rightarrow 1}$. Der Kehrwert der Halbwertsbreite des Laserpulses wird hierbei als $\gamma$ bezeichnet. Für die Population des $\mathrm{S}_{1}$-Zustands gilt dann folgendes Geschwindigkeitsgesetz:

$$
\frac{d\left[S_{1}\right]}{d t}=k_{2 \rightarrow 1} \cdot\left[S_{2}\right]-k_{1 \rightarrow 0} \cdot\left[S_{1}\right] .
$$

Aus dieser Gleichung wird die Be- und Entvölkerung des $S_{1}$ Zustandes ersichtlich. Der Kehrwert von der Geschwindigkeitskonstanten $k_{1 \rightarrow 0}$ stellt die oben genannte Lebensdauer $\tau_{1}$ dar, die von der Polarität des Lösungsmittels abhängt. Die Population des $\mathrm{S}_{0}$-Zustands folgt nachstehender formalen Kinetik:

$$
\frac{d\left[S_{0}\right]}{d t}=k_{1 \rightarrow 0} \cdot\left[S_{1}\right]-k_{A} \cdot \exp \left(-\frac{t^{2}}{\gamma^{2}}\right) .
$$

Das TA-Signal ist proportional zu den Konzentrationen im $\mathrm{S}_{1} /$ ICT- und $\mathrm{S}_{2}$-Zustand, sowie einem Beitrag der Solvensrelaxation:

$$
\text { TA-Signal }=A_{1} \cdot\left[S_{1}\right]+A_{2} \cdot\left[S_{2}\right]+A_{3} \cdot \text { Solvensrelaxation } .
$$

Für die zeitliche Abhängigkeit der Konzentrationen ergibt sich aus den Gleichungen 5.1 und 5.2 schließlich:

$$
\begin{gathered}
{\left[S_{2}\right](t)=\lambda \cdot \exp \left(-k_{2 \rightarrow 1} \cdot t\right) \int_{-\infty}^{\frac{1}{\gamma}-\frac{\gamma k_{2 \rightarrow 1}}{2}} \exp \left(-y^{2}\right) d y} \\
=\frac{\sqrt{\pi}}{2} \cdot \lambda \cdot \exp \left(-k_{2 \rightarrow 1} \cdot t\right) \cdot\left(1+\operatorname{erf}\left(\frac{2 t-\gamma^{2} k_{2 \rightarrow 1}}{2 \gamma}\right)\right) \\
{\left[S_{1}\right](t)=\frac{\sqrt{\pi}}{2} \cdot \frac{k_{2 \rightarrow 1} \lambda}{k_{2 \rightarrow 1}-k_{1 \rightarrow 0}} \cdot \exp \left(\frac{\gamma^{2}}{4}\left(k_{1 \rightarrow 0}^{2}-k_{2 \rightarrow 1}^{2}\right)\right) \cdot \exp \left(-k_{1 \rightarrow 0} t\right)} \\
\cdot\left(1+\operatorname{erf}\left(\frac{2 t-\gamma^{2} k_{1 \rightarrow 0}}{2 \gamma}\right)\right) \\
-\frac{\sqrt{\pi}}{2} \cdot \frac{k_{2 \rightarrow 1} \lambda}{k_{2 \rightarrow 1}-k_{1 \rightarrow 0}} \cdot \exp \left(\frac{\gamma^{2}}{4}\left(k_{1 \rightarrow 0}^{2}-k_{2 \rightarrow 1}^{2}\right)\right) \cdot \exp \left(-k_{1 \rightarrow 0} t\right) \cdot \exp \left(-k_{2 \rightarrow 1} t\right) \\
\cdot\left(1+\operatorname{erf}\left(\frac{2 t-\gamma^{2} k_{2 \rightarrow 1}}{2 \gamma}\right)\right)
\end{gathered}
$$


hierbei ist $\lambda=\gamma \cdot \mathrm{k}_{A} \cdot \exp \left(\frac{1}{4} \gamma^{2} \mathrm{k}_{A}^{2}\right)$.

Das Signal zeigt dann folgenden zeitlichen Verlauf:

$$
\operatorname{TA}-\operatorname{Signal}(t)=\int_{-\infty}^{\infty} \exp \left(-\left(\frac{\tau-t}{\gamma}\right)^{2}\right) \cdot\left(\alpha \cdot\left[S_{2}\right](\tau)+\beta \cdot\left[S_{1}\right](\tau)\right) d \tau
$$

Aus diesen Überlegungen ergibt sich letztendlich aus Gleichung 5.5 und 5.6 das kinetische Modell, mit dem die Signale angepasst werden:

$$
\begin{aligned}
T A-\operatorname{Signal}(t) & =A_{1} \cdot\left(1+\operatorname{erf}\left(\frac{\left(t-t_{0}\right)-\gamma^{2} / \tau_{1}}{\sqrt{2} \gamma}\right)\right) \exp \left(-\frac{t-t_{0}}{\tau_{1}}\right) \\
& +A_{2} \cdot\left(1+\operatorname{erf}\left(\frac{\left(t-t_{0}\right)-\gamma^{2} / \tau_{2}}{\sqrt{2} \gamma}\right)\right) \exp \left(-\frac{t-t_{0}}{\tau_{2}}\right) \\
& +A_{3} \cdot\left(1+\operatorname{erf}\left(\frac{\left(t-t_{0}\right)-\gamma^{2} / \tau_{3}}{\sqrt{2} \gamma}\right)\right) \exp \left(-\frac{t-t_{0}}{\tau_{3}}\right)+A_{0} .
\end{aligned}
$$

Die Solvensrelaxation wird durch eine dritte Lebensdauer $\tau_{3}$ mit der Amplitude $\mathrm{A}_{3}$ beschrieben. Es wird die gleiche mathematische Form angenommen, wie für die Relaxation $\operatorname{der} \mathrm{S}_{2}$ - und $\mathrm{S}_{1} /$ ICT-Zustände. Die Konstante $\mathrm{A}_{0}$ wird für einen möglichen Offset bei langen Zeiten eingeführt. Das Signal in Abbildung 5.1] wird mit dieser Modellfunktion angepasst. Es ist deutlich zu erkennen, dass die kinetische Modellierung gut mit den gemessenen Daten übereinstimmt.

\subsection{Ergebnisse für die Mischungen von $\left[\mathrm{C}_{6} \mathrm{mim}\right]^{+}\left[\mathrm{Tf}_{2} \mathrm{~N}\right]^{-}$mit Acetonitril}

In den folgenden Abschnitten werden die Ergebnisse der stationären Absorptions-, der PSCP- und der Zweifarben-TA-Spektroskopie von 12'-Apo- $\beta$-carotin-12'-säure (12'CA) in binären Mischungen von $\left[\mathrm{C}_{6} \mathrm{mim}\right]^{+}\left[\mathrm{Tf}_{2} \mathrm{~N}\right]^{-}$und Acetonitril präsentiert. Nach dieser Vorstellung der Ergebnisse folgt in Abschnitt 5.4 eine ausführliche Diskussion und der Vergleich mit den Messungen von $12^{\prime} \mathrm{CA}$ in $\left[\mathrm{C}_{2} \mathrm{mim}\right]^{+}\left[\mathrm{EtSO}_{4}\right]^{-}$und deren Mischungen mit Acetonitril. 


\subsubsection{Stationäre Absorptionsspektroskopie}

In der Abbildung 5.2 sind Ausschnitte der stationären Absorptionsspektren von 12' $\mathrm{CA}$ in $\left[\mathrm{C}_{6} \mathrm{mim}\right]^{+}\left[\mathrm{Tf}_{2} \mathrm{~N}\right]^{-}(-)$, Acetonitril (-) und Mischungen beider Komponenten in unterschiedlicher Zusammensetzung (graue Linien) gezeigt. Es ist jeweils eine Vergrößerung der Spektren des elektronischen Übergangs $S_{0} \rightarrow S_{2}$ abgebildet. Die Blauverschiebung von $12^{\prime} \mathrm{CA}$ in reinem $\left[\mathrm{C}_{6} \mathrm{mim}\right]^{+}\left[\mathrm{Tf}_{2} \mathrm{~N}\right]^{-}$nach Acetonitril lässt sich mit der besseren relativen Stabilisierung des $\mathrm{S}_{2}$-Zustands im Vergleich zum $\mathrm{S}_{0}$-Grundniveau in der ionischen Flüssigkeit erklären. Durch Zugabe von Acetonitril wird die $\mathrm{S}_{0}-\mathrm{S}_{2}$-Energielücke erhöht, sodass sich eine kontinuierliche Blauverschiebung der Absorptionsspektren ergibt. Dieser Effekt hängt allerdings nicht linear von der Zusammensetzung ab, wie in Abbildung 5.3 zu sehen ist. Die Abhängigkeit des Absorptionsmaximums von der Zusammensetzung nimmt mit steigendem Stoffmengenanteil der ionischen Flüssigkeit ab.

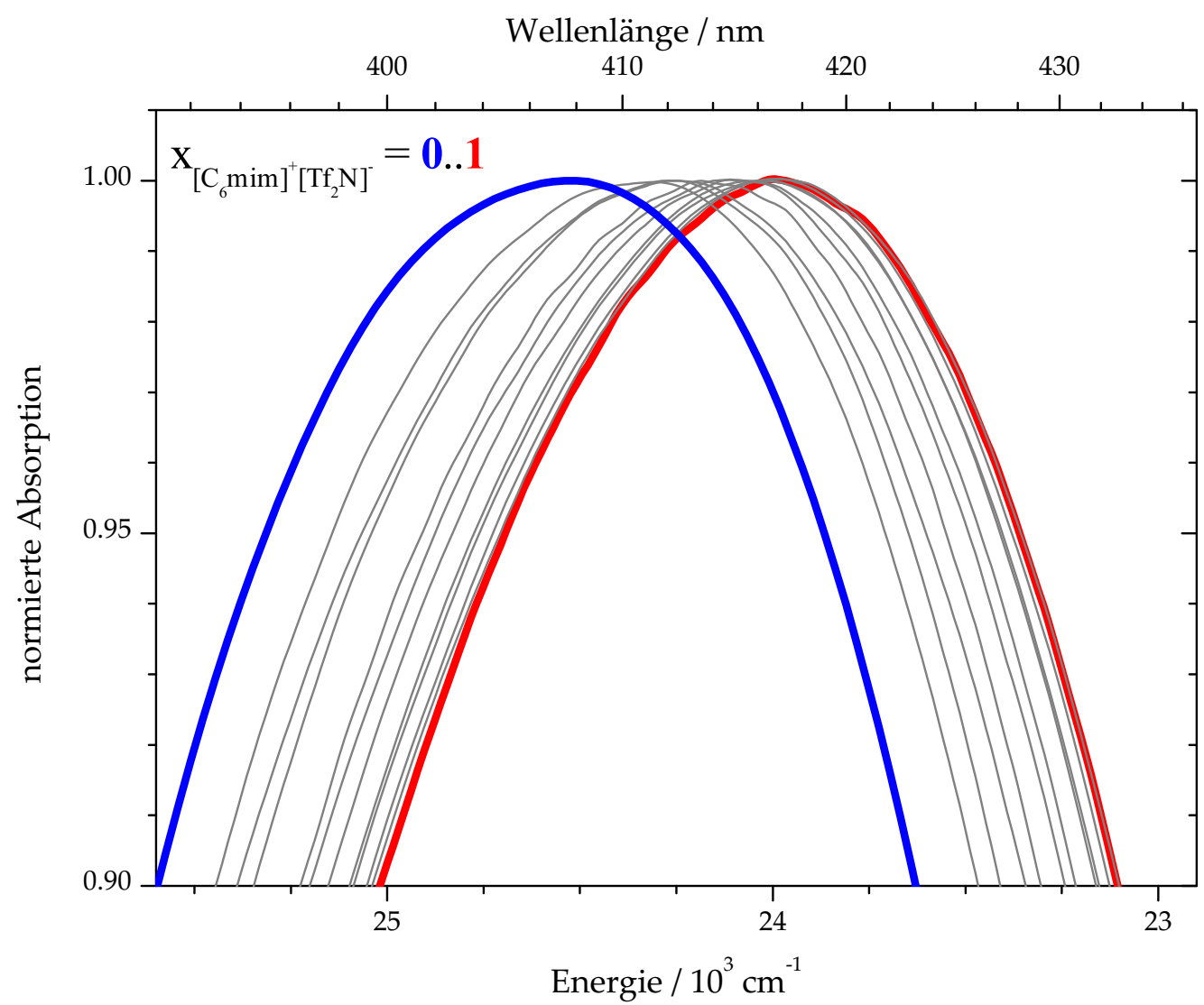

Abbildung 5.2: Stationäre Absorptionsspektren von $12^{\prime} \mathrm{CA}$ in binären Mischungen von $\left[\mathrm{C}_{6} \mathrm{mim}\right]^{+}\left[\mathrm{Tf}_{2} \mathrm{~N}\right]^{-}$und Acetonitril bei $298 \mathrm{~K}$. 


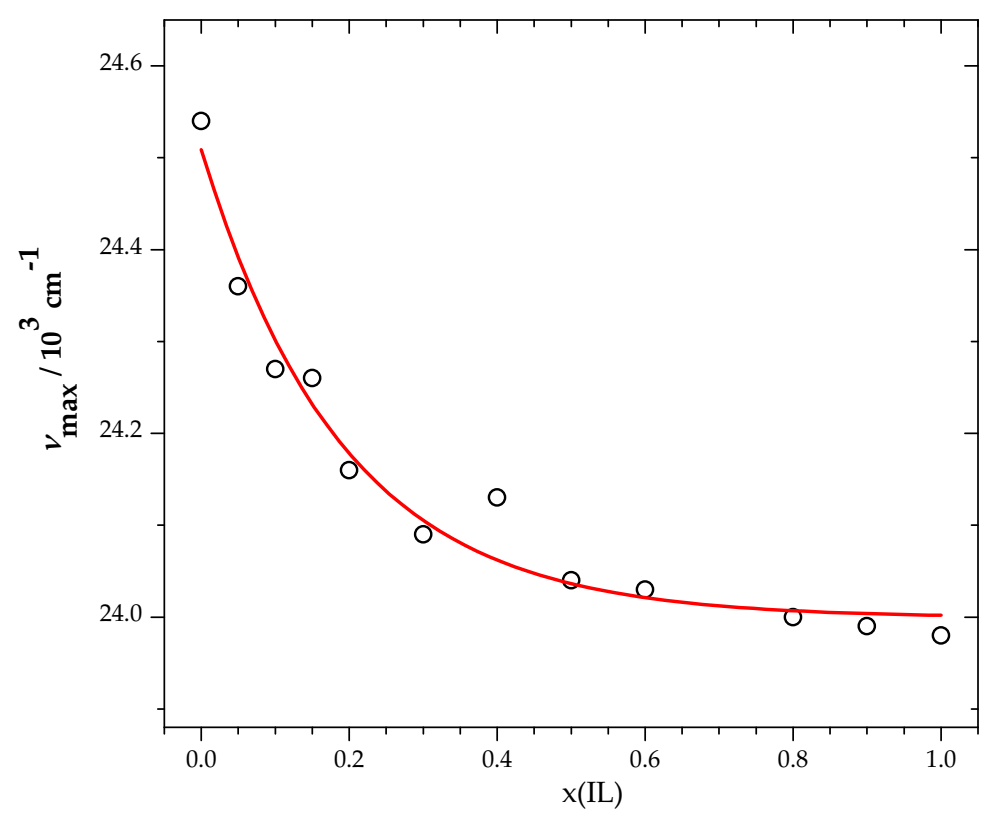

Abbildung 5.3: Abhängigkeit des stationären Absorptionsmaximums der Sonde 12' $\mathrm{CA}$ von der Zusammensetzung in binären Mischungen von $\left[\mathrm{C}_{6} \mathrm{mim}\right]^{+}\left[\mathrm{Tf}_{2} \mathrm{~N}\right]^{-}$und Acetonitril.

Die Position des Absorptionsmaximums hängt von der Polarisierbarkeit des Lösungsmittels ab. ${ }^{122}$ Diese Korrelation wird durch die Lorentz-Lorenz-Funktion beschrieben,

$$
R(n)=\left(n^{2}-1\right) /\left(n^{2}+2\right)
$$

$n$ ist hierbei der Brechungsindex des Lösungsmittels. ${ }^{122[123}$ In einem Lösungsmittel mit gröBerem $n$ hat das Carotinoid sein Absorptionsmaximum bei geringerer Wellenzahl. Bei den Mischungen mit unterschiedlicher Zusammensetzung ergibt sich ein linearer Zusammenhang zwischen dem Absorptionsmaximum von 12'CA und der Polarisierbarkeit des jeweiligen Lösungsmittels, worauf in der Diskussion noch näher eingegangen wird (siehe Abbildung 5.27).

In organischen Lösungsmitteln kann ebenfalls eine Blauverschiebung des Absorptionsmaximums von 12 'CA mit abnehmender Polarisierbarkeit beobachtet werden. ${ }^{81}$ Hierbei ergibt sich eine Verbreiterung der Absorptionsbanden mit steigender Polarität des Lösungsmittels. Dies trifft auf die Messungen der binären Mischungen nicht zu. Alle Absorptionsbanden schwanken unkorreliert mit $\pm 150 \mathrm{~cm}^{-1}$ um einen Mittelwert der Halbwertsbreiten von $4900 \mathrm{~cm}^{-1}$. 


\subsubsection{Pump-Superkontinuum Probe (PSCP)-Spektroskopie}

Eine Auswahl transienter Spektren von $12^{\prime} \mathrm{CA}$ in binären Mischungen von $\left[\mathrm{C}_{6} \mathrm{mim}\right]^{+}\left[\mathrm{Tf}_{2} \mathrm{~N}\right]^{-}$ mit Acetonitril nach Anregung bei $480 \mathrm{~nm}$ ist in den Abbildungen 5.4 bis 5.7 gezeigt. Die transienten Spektren von reiner $\left[\mathrm{C}_{6} \mathrm{mim}\right]^{+}\left[\mathrm{Tf}_{2} \mathrm{~N}\right]^{-}$ist im vorhergehenden Kapitel gezeigt worden. Abbildung 5.8 beinhaltet die Spektren von 12'CA in reinem Acetonitril. Im oberen Teil ist jeweils die Entwicklung bei kurzen Zeiten mit 20-fs-Schritten bzw. 40-fs-Schritten in Abbildung 5.8 zu sehen. Negative Signale ergeben sich aus dem Ausbleichen des Grundzustands (Ground State Bleach: GSB) und positive Signale werden der Absorption aus den angeregten Zuständen (Excited State Absorption: ESA) zugeschrieben. Die Qualität dieser frühen Signale ist aufgrund der allgemein niedrigen Intensität und Schwierigkeiten beim Abziehen des reinen Lösungsmittelsignals nicht besonders gut. Besonders auffällig ist die Struktur zwischen 450 und $600 \mathrm{~nm}$ welche als Artefakt beim Abziehen des Lösungsmittelsignals entsteht. Dennoch wird in allen Fällen die Bildung des GSB durch die $S_{0} \rightarrow S_{2^{-}}$ Absorption und der ESA durch $\mathrm{S}_{2} \rightarrow \mathrm{S}_{n}$-Absorption deutlich.

Im mittleren Bild ist die Entwicklung der transienten Spektren von wenigen fs bis 10 ps gezeigt. Es ist eine Abnahme der ESA-Bande bei etwa $700 \mathrm{~nm}$ beobachtbar, welche der Absorption aus dem zweiten elektronisch angeregten Zustand zuzuordnen ist. Gleichzeitig entwickelt sich die $\mathrm{S}_{1} /$ ICT-Bande, die in zwei Banden bei etwa 510 und $610 \mathrm{~nm}$ aufgeteilt ist. Diese Struktur wird durch die $S_{1} /$ ICT-ESA in verschiedene höhere elektronische Zustände verursacht. Das transiente Spektrum bei 10 ps verdeutlicht durch einen zusätzlichen Peak bei etwa 740 nm die Bildung eines Radikalkations von 12'CA. Dieses Radikal wird durch Zwei-Photonen-Ionisation des Pumpstrahls gebildet.

Das untere Feld umfässt jeweils die zeitliche Entwicklung von 10 ps bis mehreren 100 ps. Zusatzlich ist das stationäre Absorptionsspektrum als gestrichelte Linie gezeigt. Die Intensität der $\mathrm{S}_{1}$ /ICT ESA Bande nimmt mit der Zeit ab, wohingegen der GSB wieder aufgefüllt wird. Diese zeitliche Entwicklung der Banden wird durch sukzessive Zugabe von Acetonitril beschleunigt. Ein kleiner Rest des GSB bleibt auch bei langen Zeiten erhalten. Dies kann darauf zurückgeführt werden, dass ein Teil der $\mathrm{S}_{0}$-Population von 12 'CA in Radikalkationen umgewandelt wird. In den transienten Spektren bei langen Zeiten ist zu beachten, dass eine zusätzliche Struktur bei etwa $480 \mathrm{~nm}$ durch Schwierigkeiten bei der Streulichtkorrektur verursacht wird. 


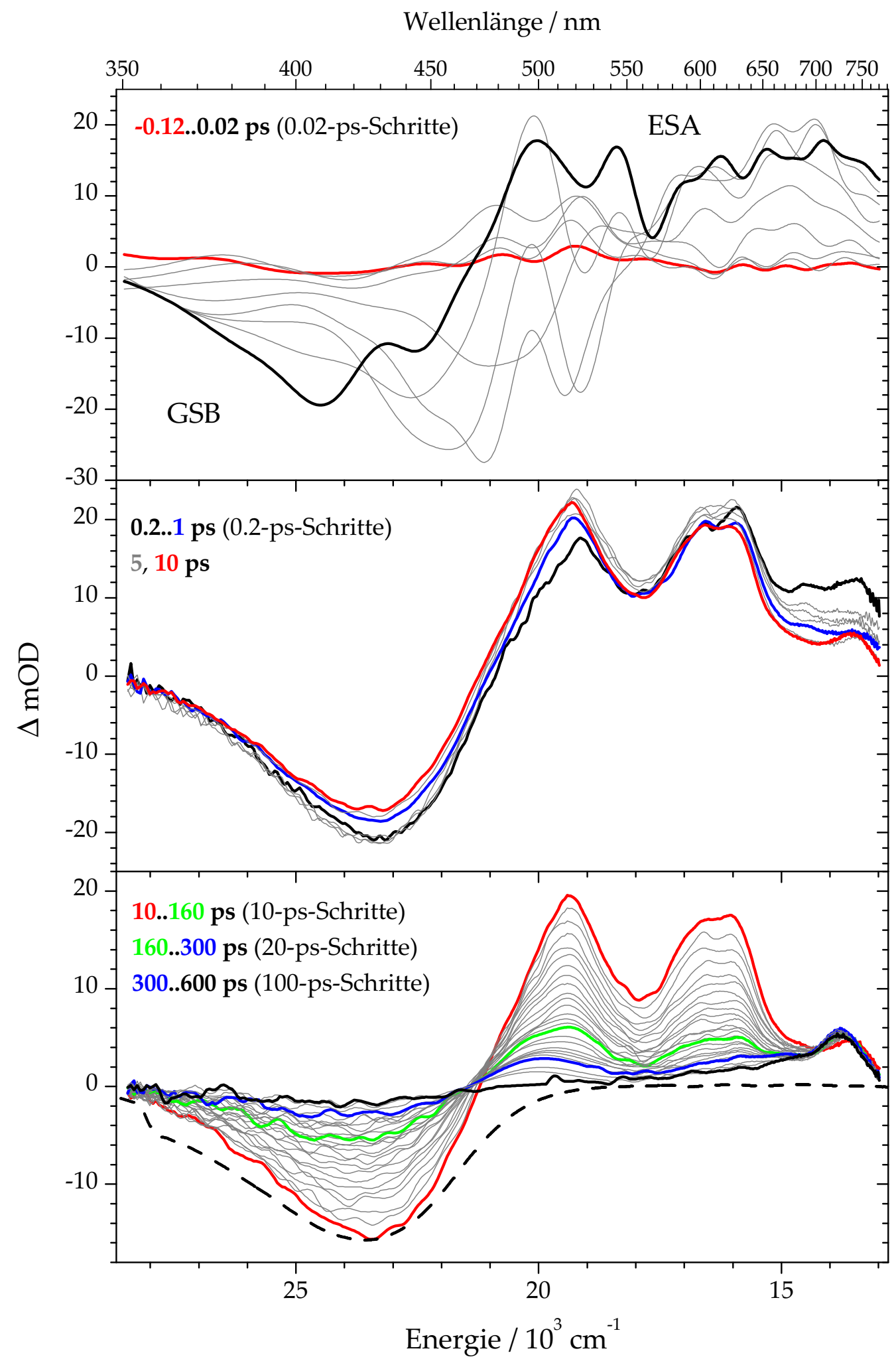

Abbildung 5.4: Transientes PSCP-Absorptionsspektrum von $12^{\prime} \mathrm{CA}$ in einer Mischung von $\left[\mathrm{C}_{6} \mathrm{~m}\right.$ $\mathrm{im}]^{+}\left[\mathrm{Tf}_{2} \mathrm{~N}\right]^{-}$mit Acetonitril $\left(\mathrm{x}_{I L}=0.75\right)$ bei einer Anregungswellenlänge von $480 \mathrm{~nm} . \Delta \mathrm{mOD}$ ist die Änderung der optischen Dichte in $10^{-3}$. (- - -) Stationäres Absorptionsspektrum. 


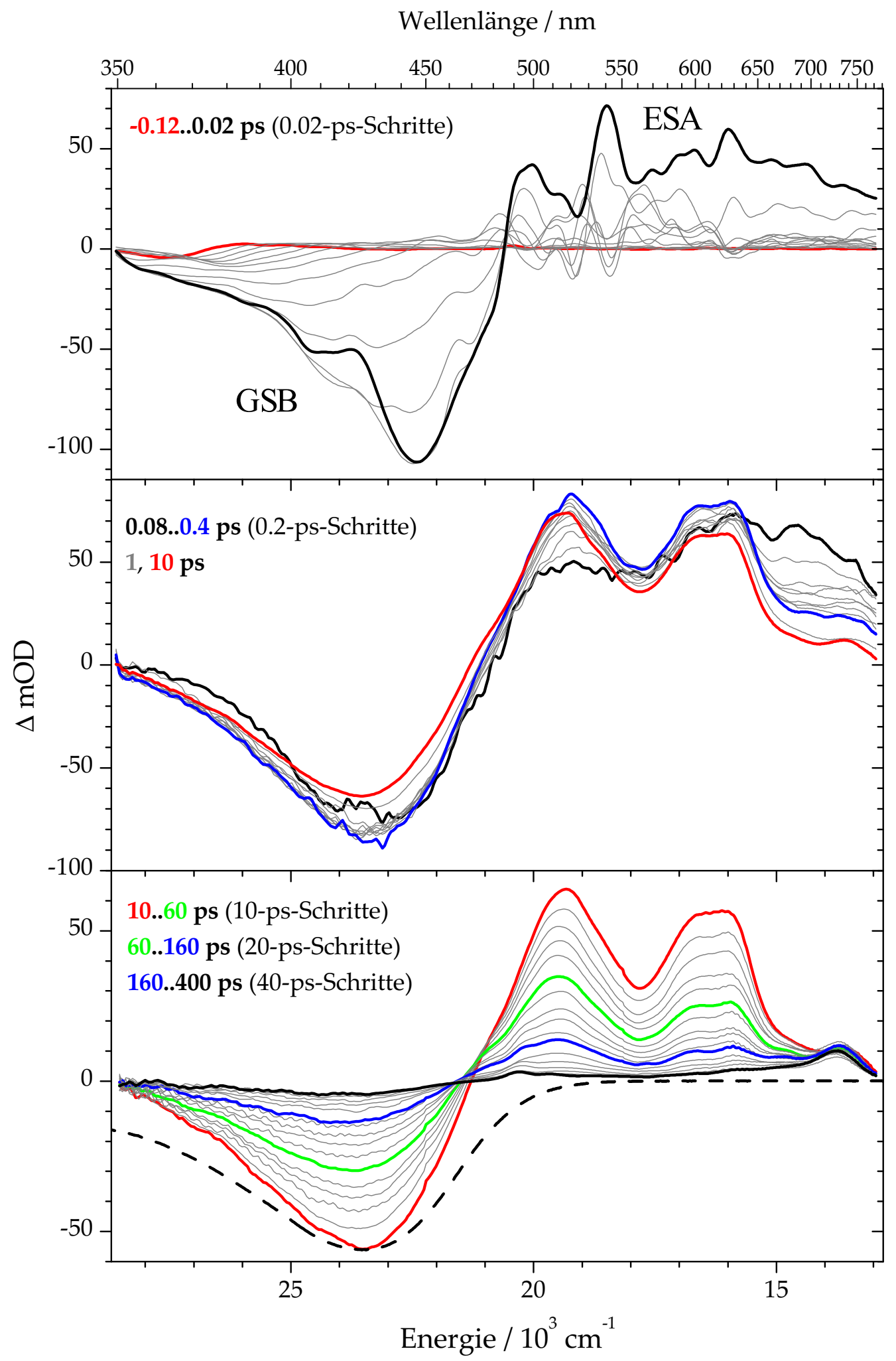

Abbildung 5.5: Wie Abb. 5.4, aber für $\mathrm{x}_{I L}=0.5$. 


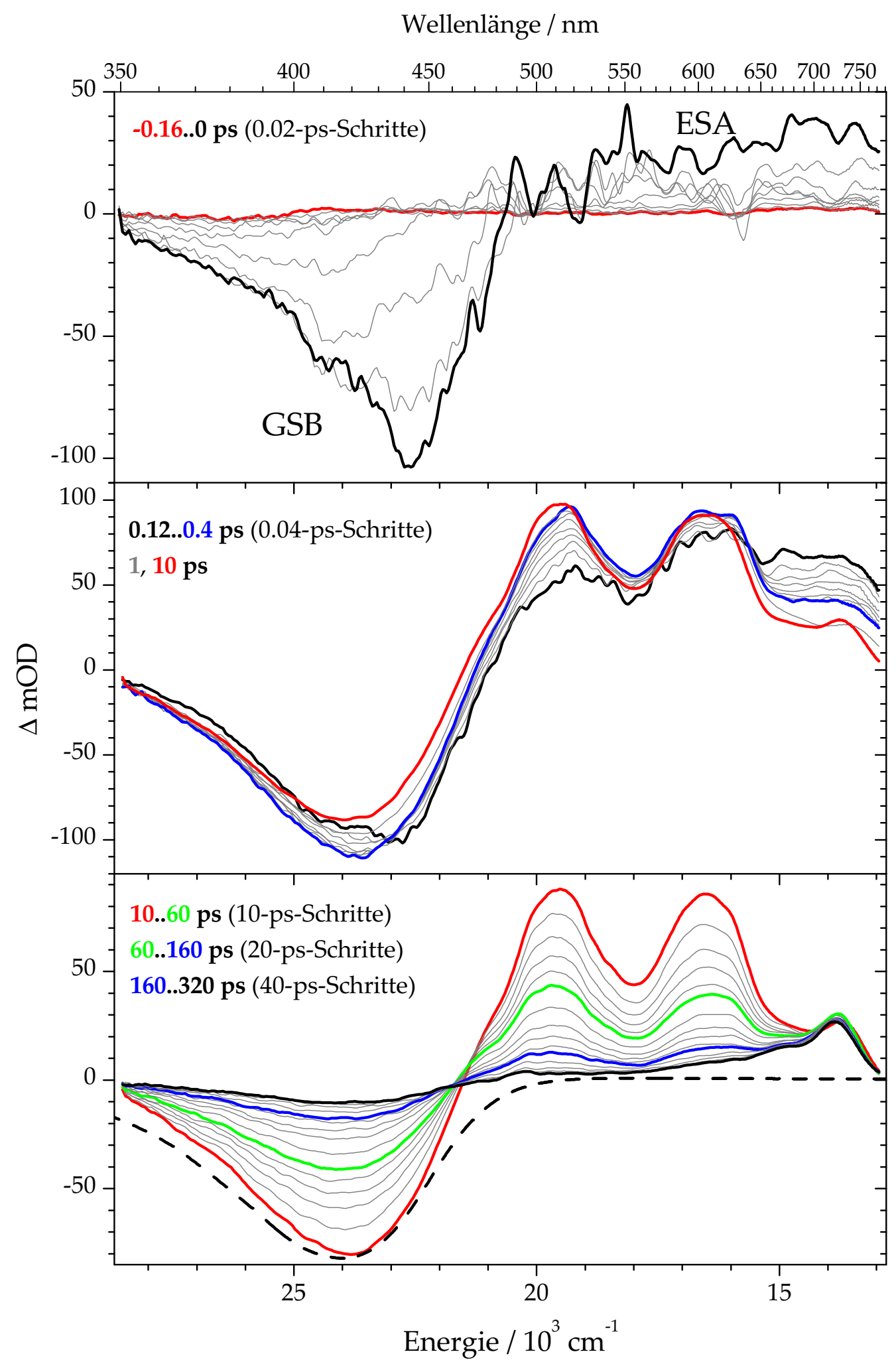

Abbildung 5.6: Wie Abb. 5.4, aber für $\mathrm{x}_{I L}=0.25$. 


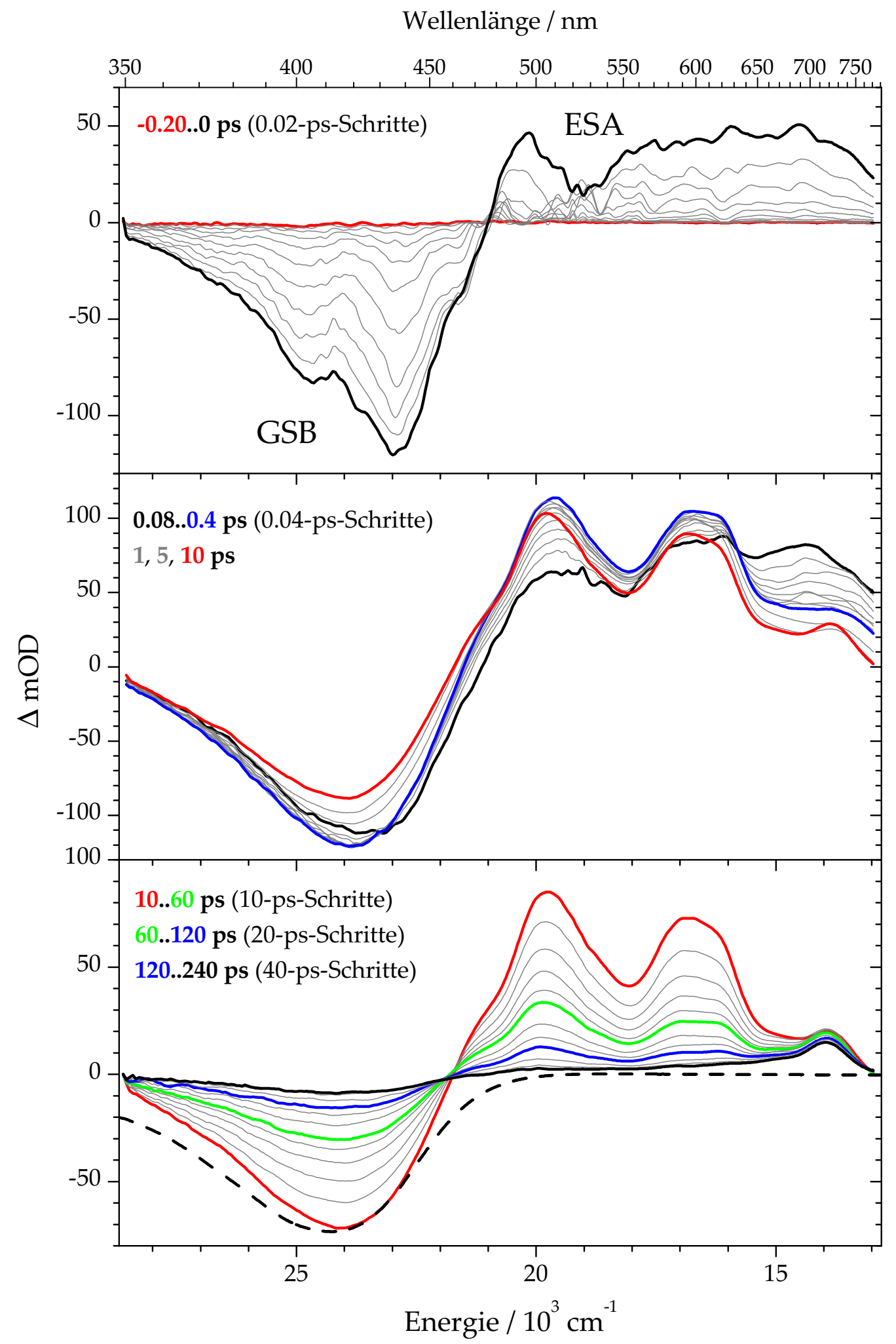

Abbildung 5.7: Wie Abb. 5.4, aber für $\mathrm{x}_{I L}=0.1$. 


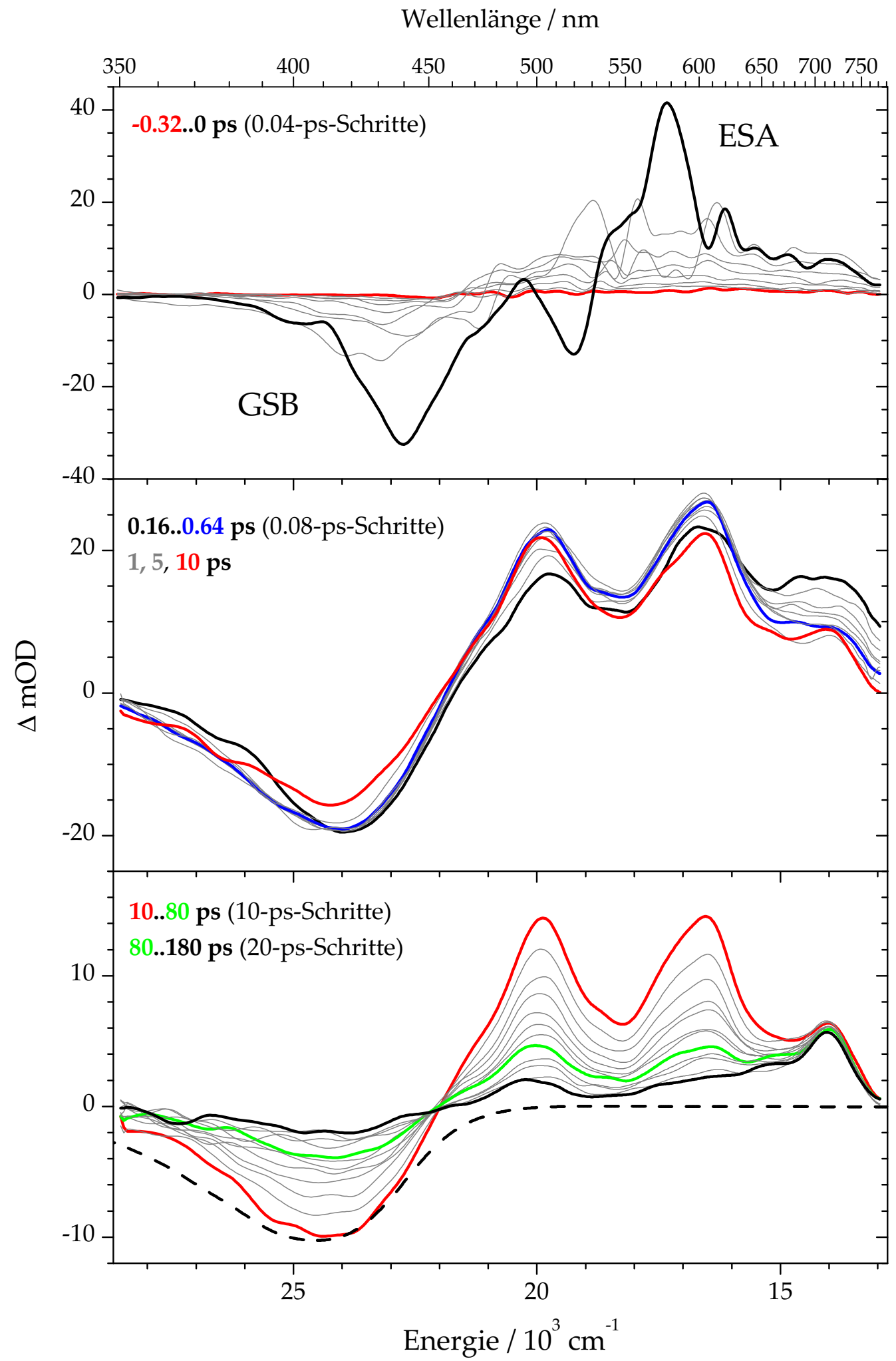

Abbildung 5.8: Wie Abb. 5.4 aber in reinem Acetonitril. 
Im unteren Teil der Abbildungen ist ein isosbestischer Punkt bei etwa $470 \mathrm{~nm}$ zu erkennen. An diesem Punkt haben die beiden Zustände $S_{1} /$ ICT und $S_{0}$ ein gleiches Absorptionsverhalten. Es handelt sich hierbei aber nicht um einen sehr deutlichen isosbestischen Punkt, sondern um einen leicht verzerrten. Dies spricht für eine zusätzliche Dynamik bei der Entwicklung der ESA-Banden, die durch eine Solvensrelaxation hervorgerufen werden könnte. Deutlich wird hierbei eine Blauverschiebung der linken ESA-Bande mit der Zeit. Diese Verschiebung ist ebenfalls im GSB zu erkennen, bei der rechten ESA-Bande hingegen nicht so stark. Daraus kann man schließen, dass es einen Überlapp mit einer ESA-Bande und dem GSB gibt. In der folgenden Abbildung wird die Blauverschiebung klar herausgestellt. Hierbei handelt es sich um transiente Absorptionsspektren von 12 ' $\mathrm{CA}$ in einer Mischung von $\left[\mathrm{C}_{6} \mathrm{mim}\right]^{+}\left[\mathrm{Tf}_{2} \mathrm{~N}\right]^{-}$und Acetonitril mit einem Stoffmengenanteil von 0.25. Die gezeigten Spektren sind, wie in Kapitel 4.2 vorgestellt, mit einer angemessenen Anzahl an Gaußfunktionen angepasste Signale. Die Spektren sind auf das GSB-Signal normiert worden. Es wird ein Ausschnitt der beiden ESA-Banden gezeigt.

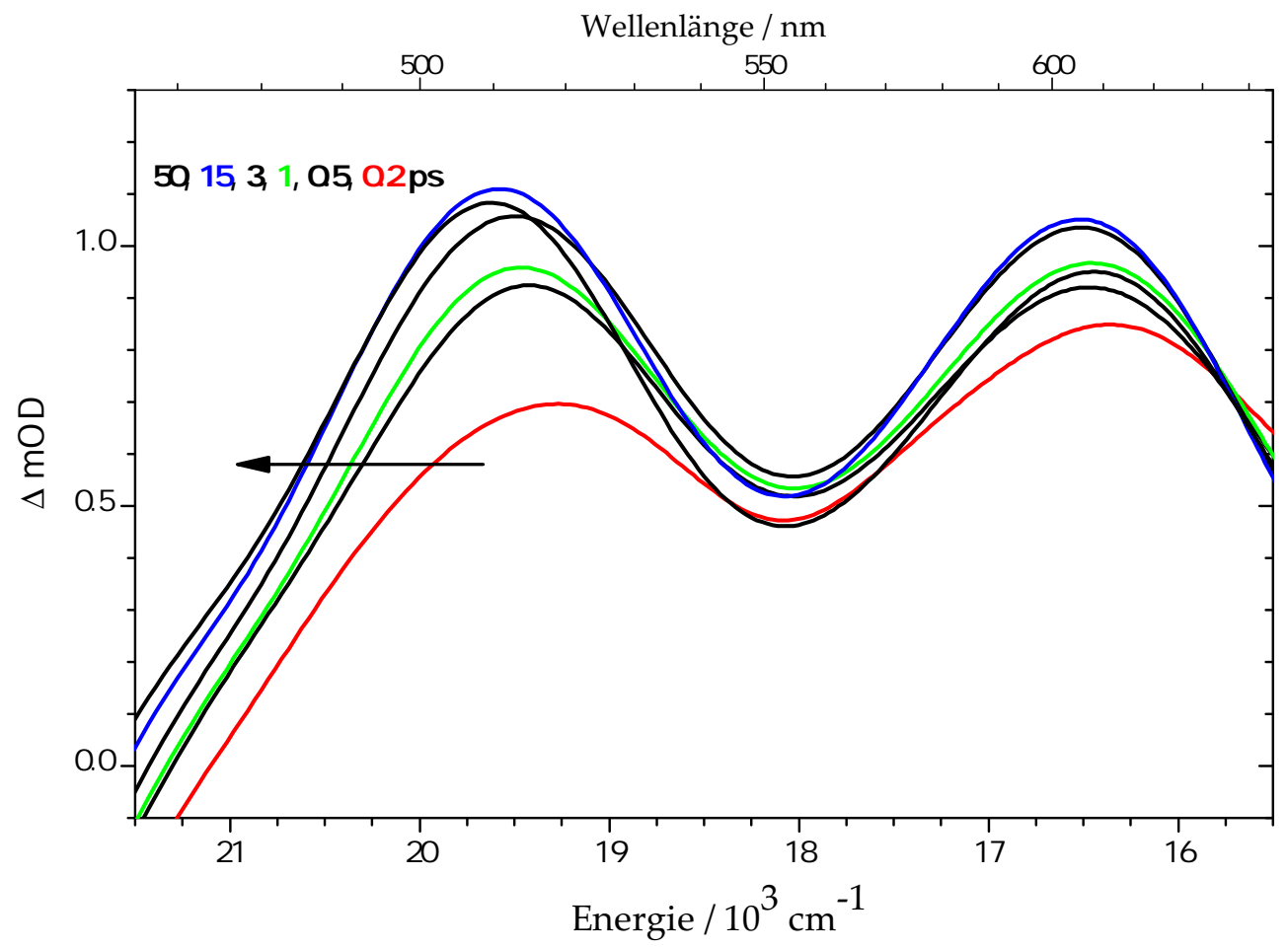

Abbildung 5.9: Ausschnitt der $\mathrm{S}_{1} /$ ICT-Banden von $12^{\prime} \mathrm{CA}$ in einer Mischung $\left[\mathrm{C}_{6} \mathrm{mim}\right]^{+}\left[\mathrm{Tf}_{2} \mathrm{~N}\right]^{-}$ und Acetonitril $(\mathrm{x}=0.25)$. 
Die Blauverschiebung kann quantitativ erfasst werden, indem die transienten Absorptionsmaxima der linken ESA-Bande gegen die Zeit aufgetragen werden. Die Daten lassen sich dann mit Gleichung 4.2 anpassen. Hierbei handelt es sich um eine Summe aus einer monoexponentiellen und einer gestrecktexponentiellen Funktion. Die Solvensdynamik, die für die Blauverschiebung verantwortlich ist, lässt sich somit durch eine langsame Komponente $\left(\tau_{l}\right)$ und eine schnelle Komponente $\left(\tau_{s}\right)$ beschreiben. Hierbei tritt $\tau_{l}$ im gestrecktexponentiellen Teil und $\left(\tau_{s}\right)$ im monoexponentiellen Teil auf. Der genaue Hintergrund ist in Kapitel 4 beschrieben. Exemplarisch ist eine solche Anpassung in Abbildung 5.10 aufgeführt. Hierbei handelt es sich um die linke ESA-Bande von 12'CA in einer Mischung von $\left[\mathrm{C}_{6} \mathrm{mim}\right]^{+}\left[\mathrm{Tf}_{2} \mathrm{~N}\right]^{-}$und Acetonitril mit einem Molenbruch von $\mathrm{x}_{I L}=0.1$. Die Anpassungsparameter sind Tabelle $5.1 \mathrm{zu}$ entnehmen.

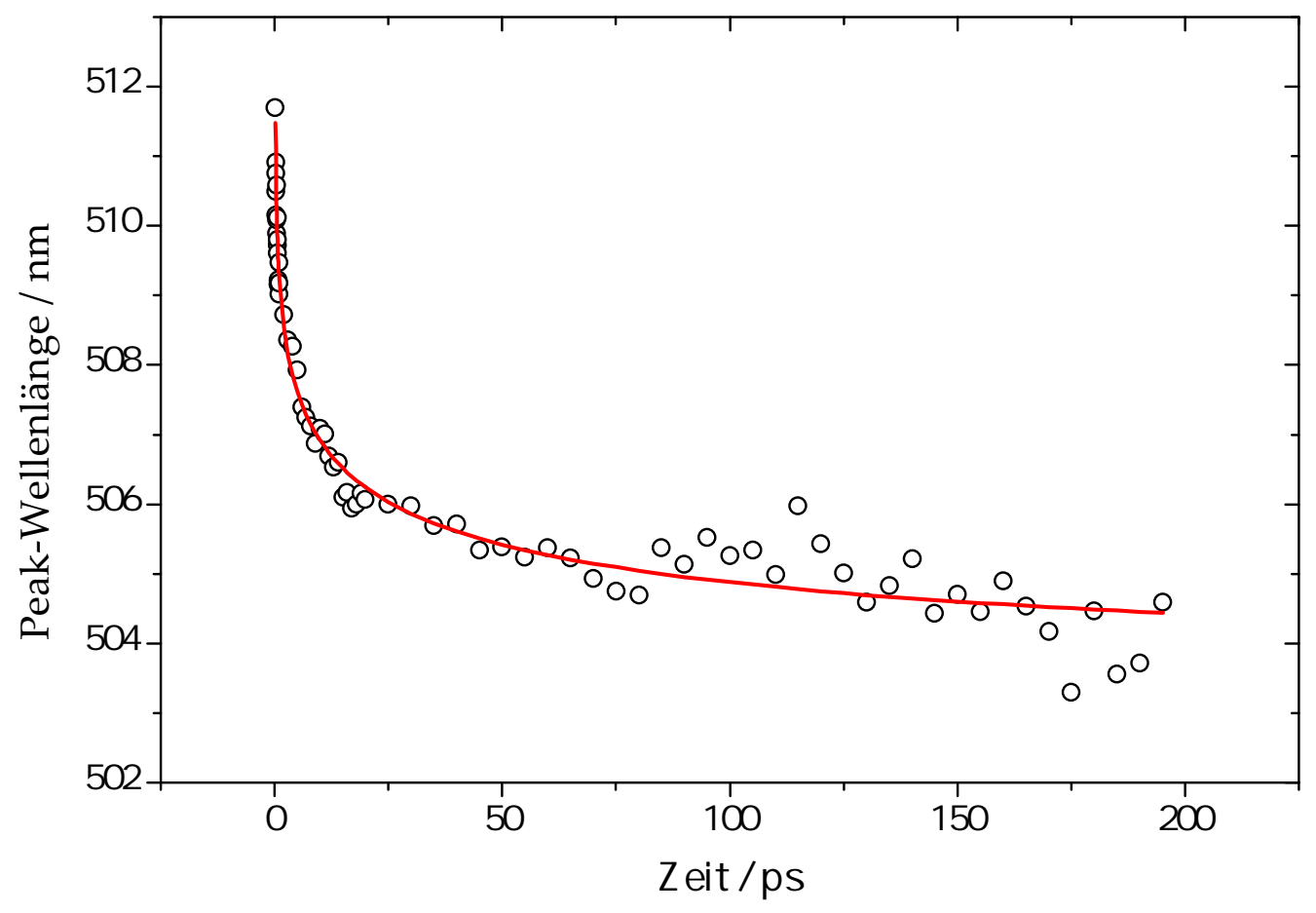

Abbildung 5.10: Anpassung der spektralen Entwicklung der linken ESA-Bande von 12'CA in einer Mischung von $\left[\mathrm{C}_{6} \mathrm{mim}\right]^{+}\left[\mathrm{Tf}_{2} \mathrm{~N}\right]^{-}$und Acetonitril $(\mathrm{x}=0.1)$.

Den PSCP-Daten können kinetische Spuren entnommen werden. So kann durch eine globale Analyse die Lebensdauer des $\mathrm{S}_{1}$ / ICT-Zustands bestimmt werden (siehe Kapitel 4.2). Da diese Lebensdauer von der Art des Lösungsmittels abhängig ist, können Rückschlüsse auf die Mikrostruktur der ionischen Flüssigkeiten und derer binären Mischungen gezogen 
werden. Vier exemplarische kinetische Spuren sind für jede binäre Mischung und reines Acetonitril in den Abbildungen 5.11 bis 5.15 dargestellt. Die kinetischen Spuren für $\left[\mathrm{C}_{6} \mathrm{~m}\right.$ $\mathrm{im}]^{+}\left[\mathrm{Tf}_{2} \mathrm{~N}\right]^{-}$sind bereits in Kapitel 4.3 gezeigt worden (siehe Abbildung 4.5). Ebenfalls den Abbildungen zu entnehmen ist eine Anpassung der Signale. Hierbei hat sich bei einer globalen Analyse gezeigt, dass sich die PSCP-Daten am besten durch eine biexponentielle Funktion simulieren lassen. Die Zeitkonstanten der biexponentiellen Anpassung ( $\tau_{1, A}$ und $\left.\tau_{1, B}\right)$ für die innere Konversion $\mathrm{S}_{1} / \mathrm{ICT} \rightarrow \mathrm{S}_{0}$ sind in Tabelle 5.1 zusammengefasst.

Tabelle 5.1: Anpassungsparameter

\begin{tabular}{lccccc}
\hline Lösungsmittel & $\tau_{1, A}$ [ps] & $\tau_{1, B}$ [ps] & $\tau_{l}$ [ps] & $\tau_{s}$ [ps] & $\beta$ \\
\hline $\left.\mathrm{CC}_{6} \mathrm{mim}\right]^{+}\left[\mathrm{Tf}_{2} \mathrm{~N}\right]^{-}$ & 158 & 45 & 32 & 0.1 & 0.33 \\
$\mathrm{x}_{I L}=0.75$ & 126 & 49 & 65 & 0.1 & 0.37 \\
$\mathrm{x}_{I L}=0.5$ & 110 & 32 & 17 & 0.1 & 0.47 \\
$\mathrm{x}_{I L}=0.25$ & 84 & 29 & 103 & 0.2 & 0.26 \\
$\mathrm{x}_{I L}=0.1$ & 79 & 30 & 4.4 & 0.2 & 0.23 \\
Acetonitril & 70 & 17 & 4.1 & 1.6 & 0.48 \\
\hline
\end{tabular}

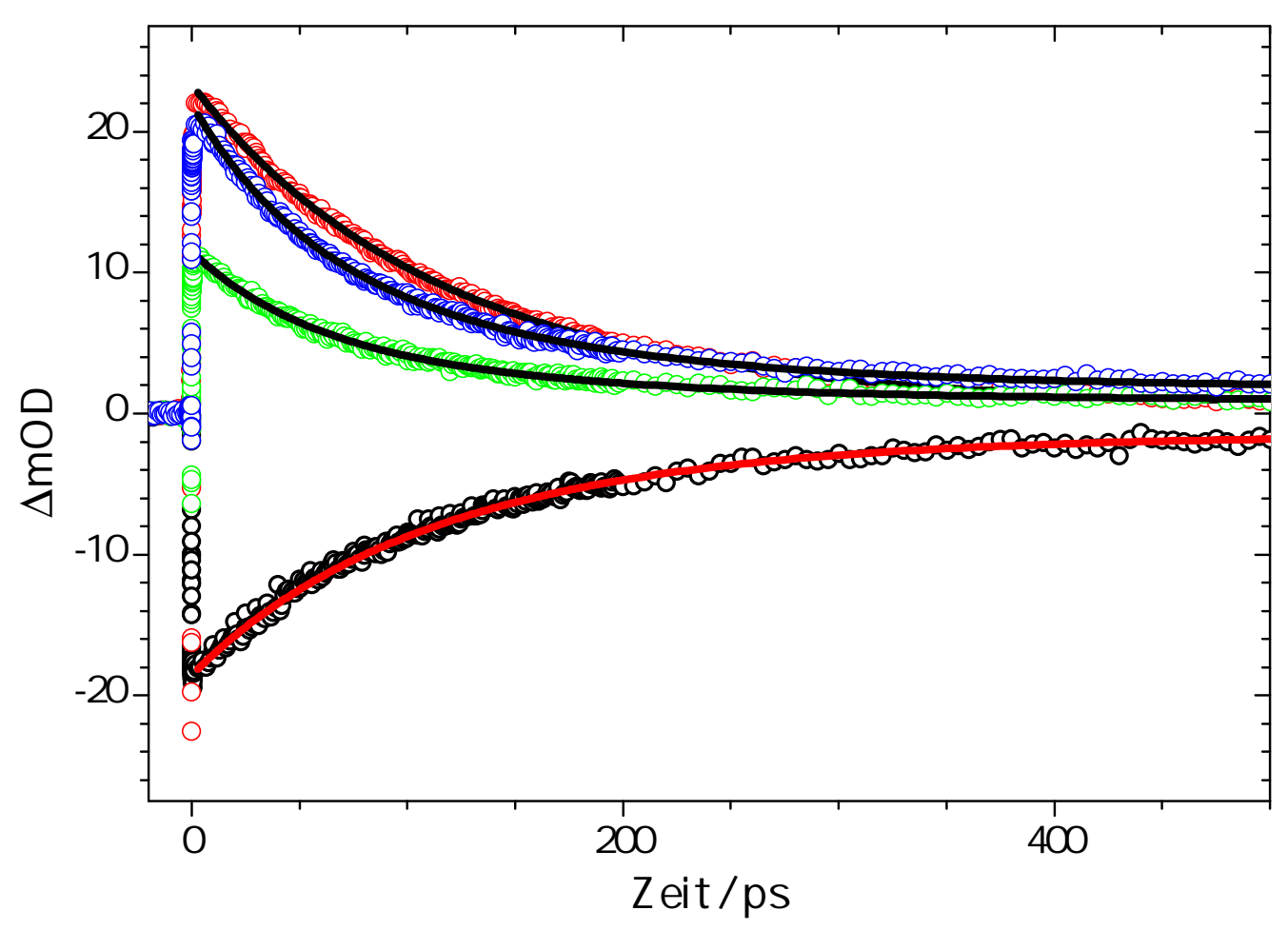

Abbildung 5.11: Kinetische Spuren mit globaler Anpassung von $12^{\prime} \mathrm{CA}$ in einer Mischung von $\left[\mathrm{C}_{6} \mathrm{mim}\right]^{+}\left[\mathrm{Tf}_{2} \mathrm{~N}\right]^{-}$und Acetonitril $(\mathrm{x}=0.75)$ bei vier repräsentativen Wellenlängen. (०) $423 \mathrm{~nm}$, (०) $522 \mathrm{~nm},(\circ) 563 \mathrm{~nm}$ und (०) $620 \mathrm{~nm}$. 


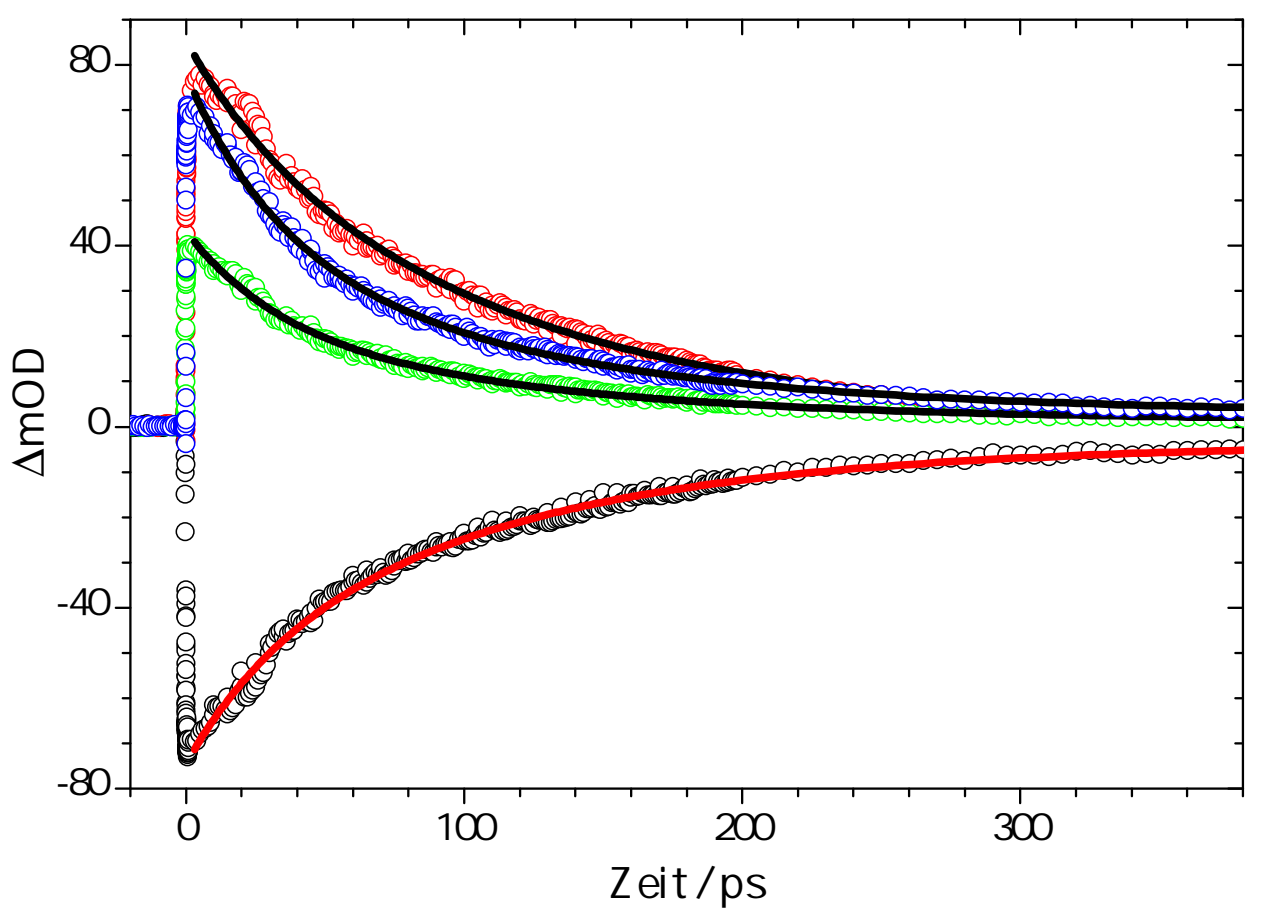

Abbildung 5.12: Kinetische Spuren mit globaler Anpassung von $12^{\prime} \mathrm{CA}$ in einer Mischung von $\left[\mathrm{C}_{6} \mathrm{mim}\right]^{+}\left[\mathrm{Tf}_{2} \mathrm{~N}\right]^{-}$und Acetonitril $(\mathrm{x}=0.5)$ bei vier repräsentativen Wellenlängen. (०) $423 \mathrm{~nm}$, (०) $514 \mathrm{~nm},(\circ) 564 \mathrm{~nm}$ und (०) $622 \mathrm{~nm}$.

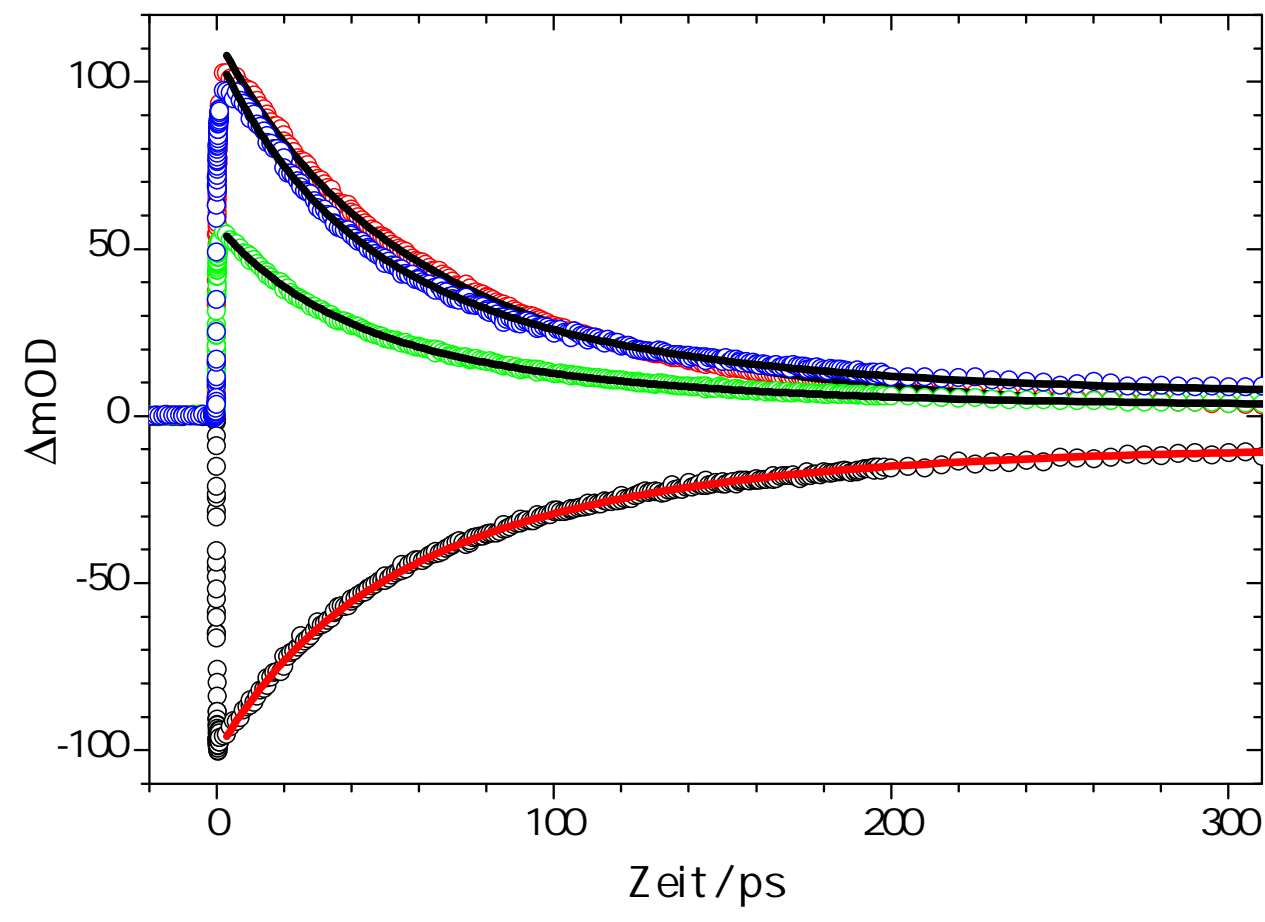

Abbildung 5.13: Kinetische Spuren mit globaler Anpassung von 12' $\mathrm{CA}$ in einer Mischung von $\left[\mathrm{C}_{6} \mathrm{mim}\right]^{+}\left[\mathrm{Tf}_{2} \mathrm{~N}\right]^{-}$und Acetonitril $(x=0.25)$ bei vier repräsentativen Wellenlängen. (o) $423 \mathrm{~nm}$, (०) $514 \mathrm{~nm},(\circ) 556 \mathrm{~nm}$ und (०) $606 \mathrm{~nm}$. 


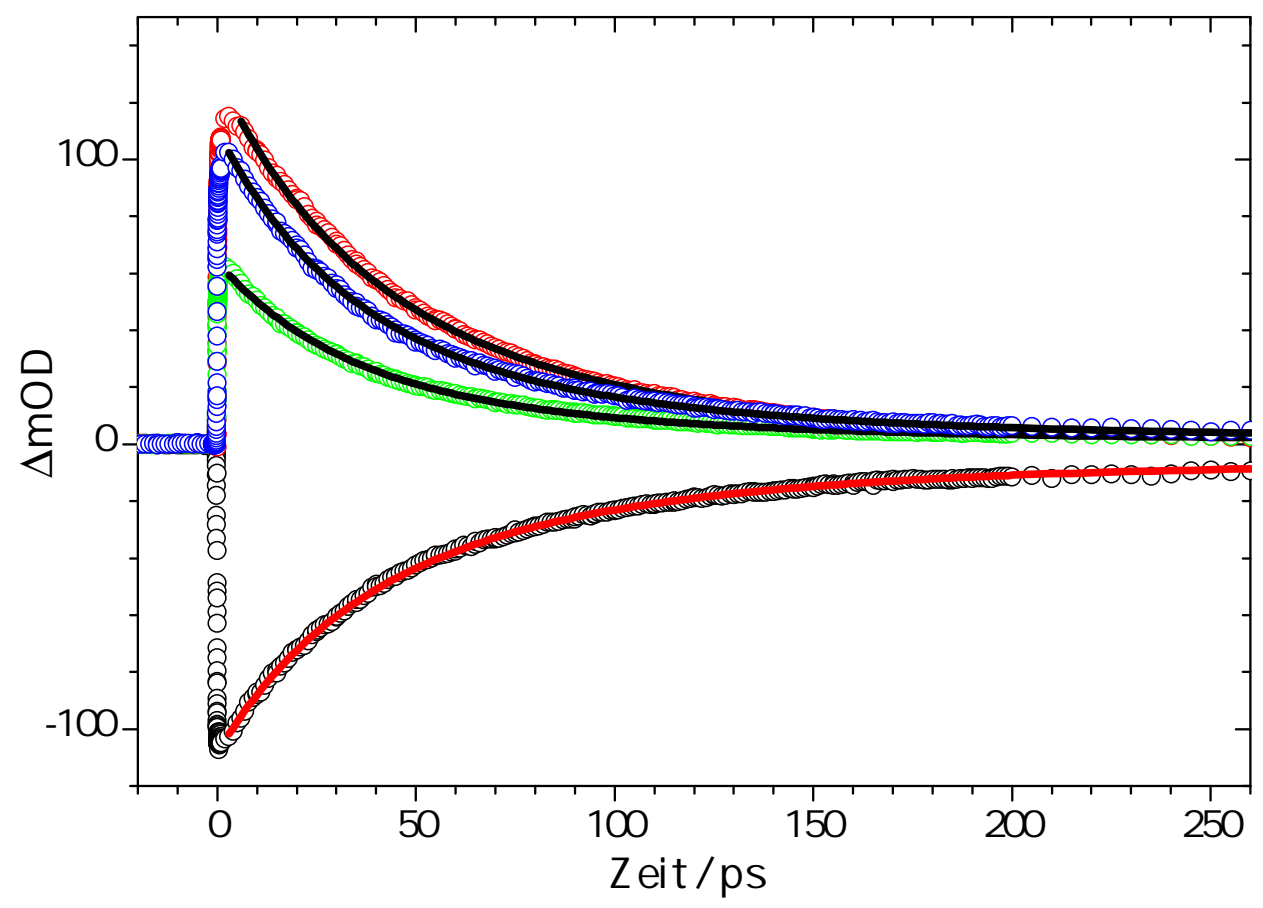

Abbildung 5.14: Kinetische Spuren mit globaler Anpassung von $12^{\prime} \mathrm{CA}$ in einer Mischung von

$\left[\mathrm{C}_{6} \mathrm{mim}\right]^{+}\left[\mathrm{Tf}_{2} \mathrm{~N}\right]^{-}$und Acetonitril $(\mathrm{x}=0.1)$ bei vier repräsentativen Wellenlängen. ( $\left.\circ\right) 415 \mathrm{~nm}$, (०) $506 \mathrm{~nm},(\circ) 556 \mathrm{~nm}$ und (०) $606 \mathrm{~nm}$.

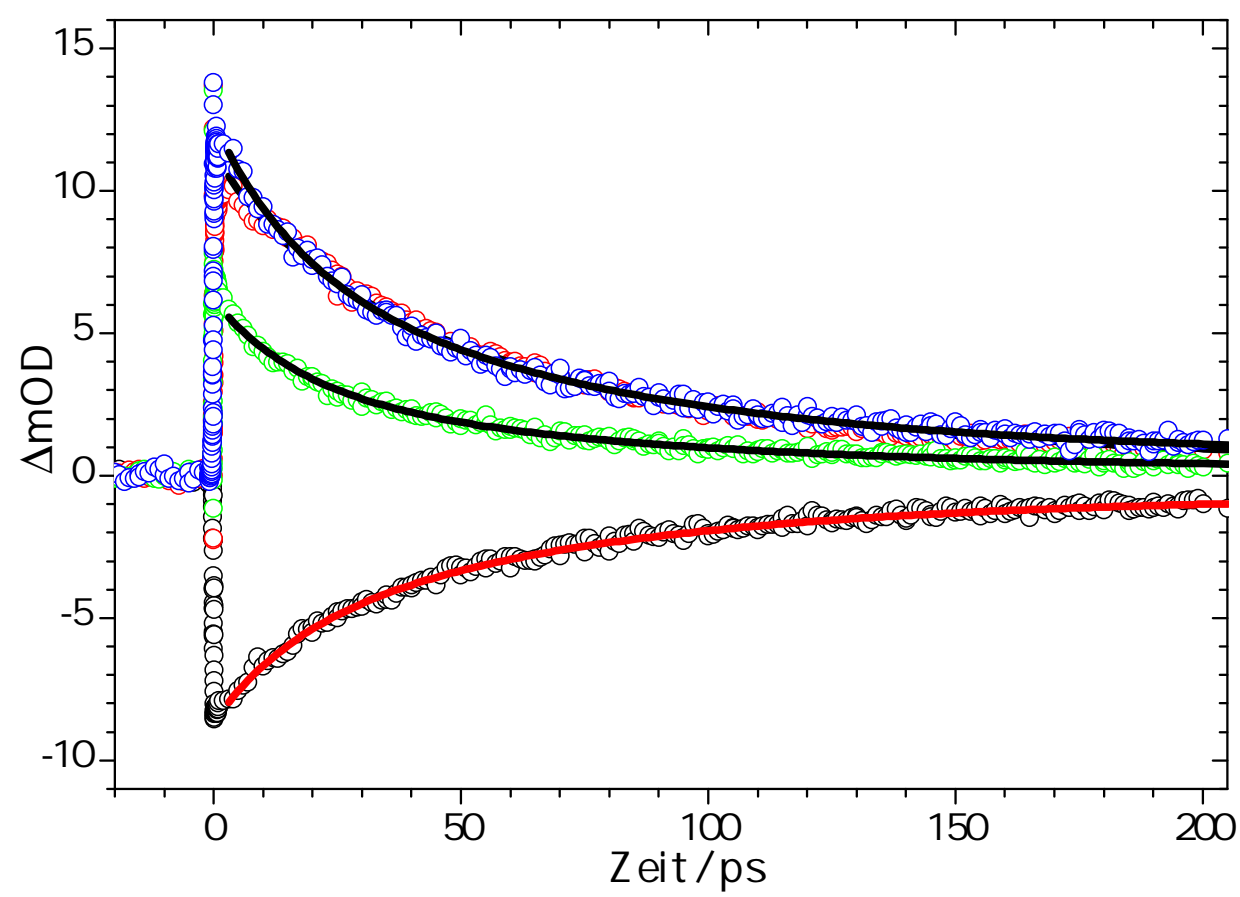

Abbildung 5.15: Kinetische Spuren mit globaler Anpassung von 12'CA in Acetonitril. (०) $415 \mathrm{~nm}$, (०) $498 \mathrm{~nm},(\circ) 548 \mathrm{~nm}$ und (०) $606 \mathrm{~nm}$. 
Analog zur stationären Absorption von $12^{\prime} \mathrm{CA}$ in $\left[\mathrm{C}_{6} \mathrm{mim}\right]^{+}\left[\mathrm{Tf}_{2} \mathrm{~N}\right]^{-}$und den Mischungen mit Acetonitril ergibt sich eine Blauverschiebung des GSB durch Zugabe des organischen Lösungsmittels. In Abbildung 5.16 sind die transienten Spektren von 12 ' $\mathrm{CA}$ in $\left[\mathrm{C}_{6} \mathrm{~m}-\right.$ $\mathrm{im}]^{+}\left[\mathrm{Tf}_{2} \mathrm{~N}\right]^{-}(-)$, Acetonitril (-) und Mischungen verschiedener Zusammensetzungen (graue Linien) bei 10 ps gezeigt. Abgesehen von der Blauverschiebung mit steigendem Stoffmengenanteil an Acetonitril fällt das unterschiedliche Verhältnis der GSB- und ESABande auf. In reinem Acetonitril ist die Intensität der ESA-Bande deutlich höher, wohingegen die Mischungen mit $\left[\mathrm{C}_{6} \mathrm{mim}\right]^{+}\left[\mathrm{Tf}_{2} \mathrm{~N}\right]^{-}$auch bei geringen Molenbrüchen ein sehr ähnliches Verhältnis zeigen.

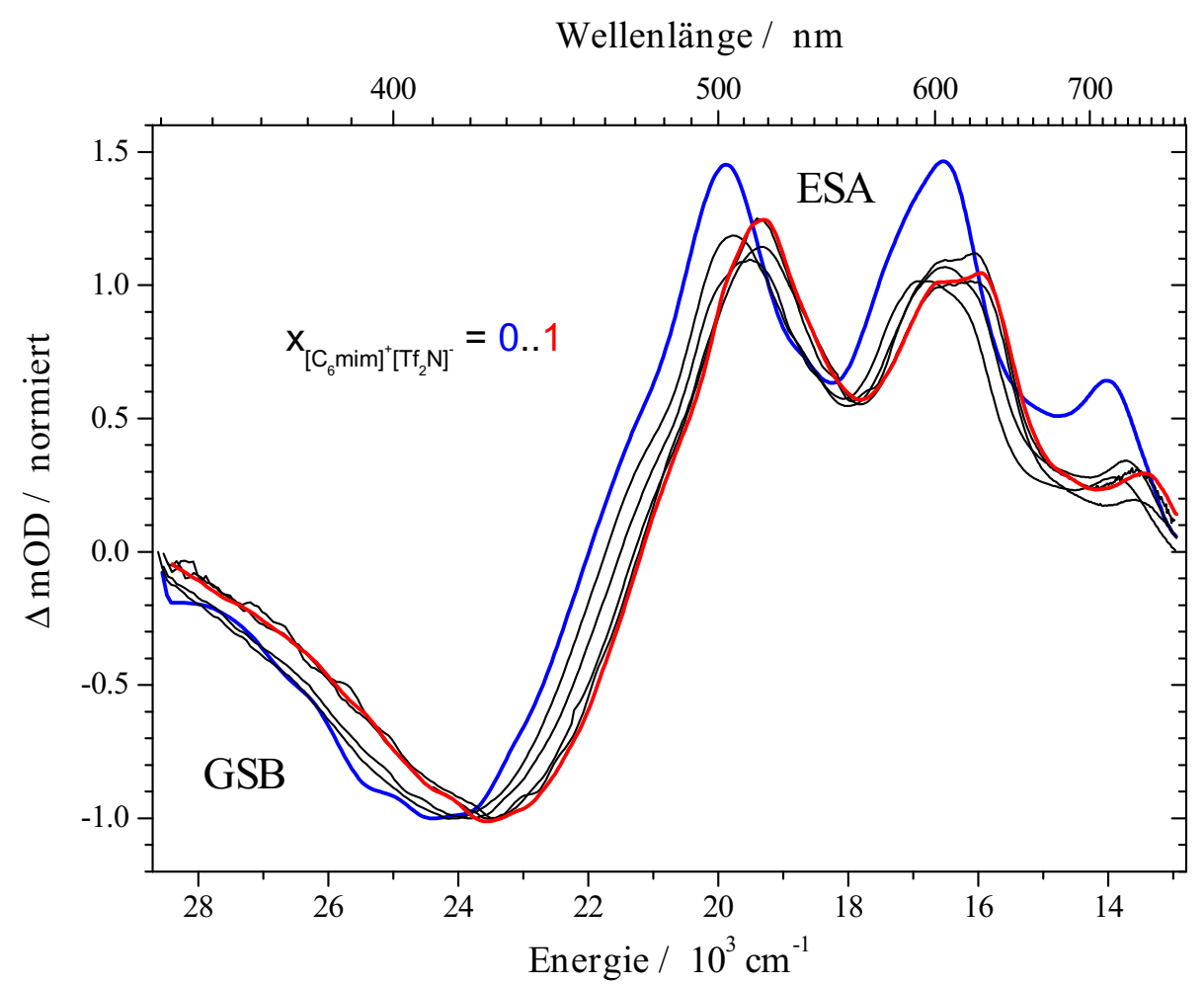

Abbildung 5.16: Transiente PSCP-Absorptionsspektren von $12^{\prime} \mathrm{CA}$ in Mischungen von $\left[\mathrm{C}_{6} \mathrm{mim}\right]^{+}$$\left[\mathrm{Tf}_{2} \mathrm{~N}\right]^{-}$und Acetonitril unterschiedlicher Zusammensetzung bei $10 \mathrm{ps.}$ 


\subsubsection{Transiente Absorptions- (TA) Spektroskopie}

Die Dynamik der inneren Konversion $\mathrm{S}_{1} / \mathrm{ICT} \rightarrow \mathrm{S}_{0}$ von $12^{\prime} \mathrm{CA}$ in den binären Mischungen ist ebenfalls durch transiente Absorptions- (TA) Spektroskopie im nahen Infrarot untersucht worden. In den Abbildungen 5.17 bis 5.20 sind die transienten Signale samt der kinetischen Modellierung nach Gleichung 5.8 (siehe Kapitel 5.1) dargestellt. Tabelle 5.2 führt die Parameter aller Anpassungen auf. Die TA-Signale beginnen alle mit einem schnellen Anstieg, welcher durch die Population des $\mathrm{S}_{2}$-Zustands hervorgerufen wird. Durch schnelle innere Konversion $S_{2} \rightarrow S_{1} /$ ICT bildet sich ein negatives Signal. Der $S_{1} /$ ICT-Zustand wird durch eine langsamere innere Konversion in den $\mathrm{S}_{0}$-Zustand abgebaut. Die Anpassungen der Signale sind in den entsprechenden Abbildungen als rote Linien gezeigt. Das Verhalten bei sehr frühen Zeiten ist bei den korrespondierenden Signalen als Ausschnitt eingefügt.

Tabelle 5.2: Anpassungsparameter

\begin{tabular}{clccccccccc}
\hline $\mathrm{Nr}$. & $\mathrm{x}_{I L}$ & $\mathrm{t}_{0}[\mathrm{ps}]$ & $\gamma[\mathrm{ps}]$ & $\mathrm{A}_{1}$ & $\tau_{1}[\mathrm{ps}]$ & $\mathrm{A}_{2}$ & $\tau_{2}[\mathrm{ps}]$ & $\mathrm{A}_{3}$ & $\tau_{3}[\mathrm{ps}]$ & $\mathrm{A}_{0}$ \\
\hline 1 & 0 & -0.03 & 0.1 & -0.5 & 42 & 5.9 & 0.1 & 0.3 & 0.4 & -0.05 \\
2 & 0.05 & -0.04 & 0.1 & -0.5 & 38 & 5.6 & 0.1 & 0.3 & 0.8 & -0.02 \\
3 & 0.1 & -0.06 & 0.1 & -0.5 & 38 & 4.3 & 0.1 & 0.3 & 1.1 & -0.02 \\
4 & 0.15 & -0.07 & 0.1 & -0.5 & 43 & 2.0 & 0.1 & 0.2 & 1.7 & -0.01 \\
5 & 0.2 & -0.05 & 0.1 & -0.5 & 45 & 2.8 & 0.1 & 0.3 & 1.3 & 0.01 \\
6 & 0.3 & -0.07 & 0.1 & -0.5 & 56 & 2.6 & 0.1 & 0.2 & 1.9 & -0.04 \\
7 & 0.4 & -0.06 & 0.1 & -0.5 & 66 & 1.9 & 0.1 & 0.2 & 2.1 & -0.04 \\
8 & 0.5 & -0.06 & 0.1 & -0.5 & 73 & 4.3 & 0.1 & 0.3 & 2.0 & -0.04 \\
9 & 0.6 & -0.05 & 0.1 & -0.5 & 83 & 3.7 & 0.1 & 0.2 & 2.8 & -0.01 \\
10 & 0.8 & -0.06 & 0.1 & -0.5 & 85 & 3.3 & 0.1 & 0.2 & 2.8 & -0.05 \\
11 & 0.9 & -0.05 & 0.1 & -0.5 & 90 & 3.2 & 0.1 & 0.2 & 2.4 & -0.03 \\
12 & 1.0 & -0.06 & 0.1 & -0.5 & 91 & 3.9 & 0.1 & 0.2 & 2.5 & -0.04 \\
\hline
\end{tabular}

Von besonderem Interesse sind die Lebensdauern $\tau_{1}$ und $\tau_{3}$ des Carotinoids, welche aus der Anpassung der Signale extrahiert werden können. Die Dauer der inneren Konversion des $\mathrm{S}_{1} /$ ICT-Zustands wird mit der Konstanten $\tau_{1}$ beschrieben. Die Bildung dieses Zustands wird durch die Konstante $\tau_{3}$ verdeutlicht. Beide Konstanten hängen von der Beschaffenheit des Lösungsmittels ab. Es sind TA-Messungen von 12'CA in binären Mischungen von $\left[\mathrm{C}_{6} \mathrm{mim}\right]^{+}\left[\mathrm{Tf}_{2} \mathrm{~N}\right]^{-}$und Acetonitril über den gesammten Zusammensetzungsbereich durchgeführt worden. Hierbei hat sich für $\tau_{1}$ ein lineare Abhängigkeit von 
der Zusammensetzung ergeben, wohingegen $\tau_{3}$ einen nicht-linearen Verlauf zeigt (siehe Diskussion, Abbildungen 5.28 und 5.29).

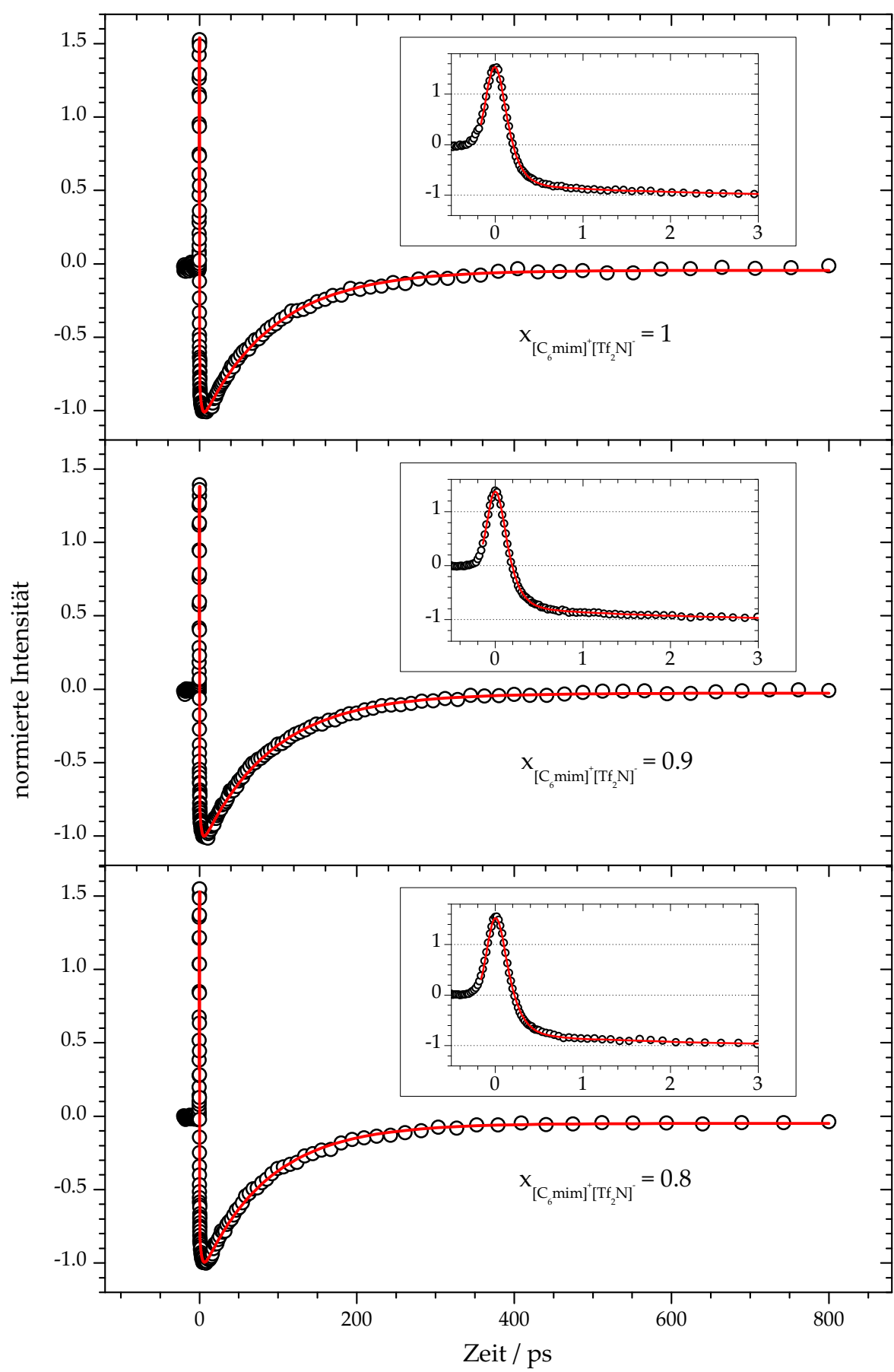

Abbildung 5.17: Transiente Absorptionssignale von $12^{\prime} \mathrm{CA}$ in Mischungen von $\left[\mathrm{C}_{6} \mathrm{mim}\right]^{+}\left[\mathrm{Tf}_{2} \mathrm{~N}\right]^{-}$ und Acetonitril mit den Zusammensetzungen $\mathrm{x}_{I L}=1, \mathrm{x}_{I L}=0.9$ und $\mathrm{x}_{I L}=0.8$ (Anregung: 430 $\mathrm{nm}$; Abfrage: $860 \mathrm{~nm}) .(-)$ : kinetische Anpassung. 


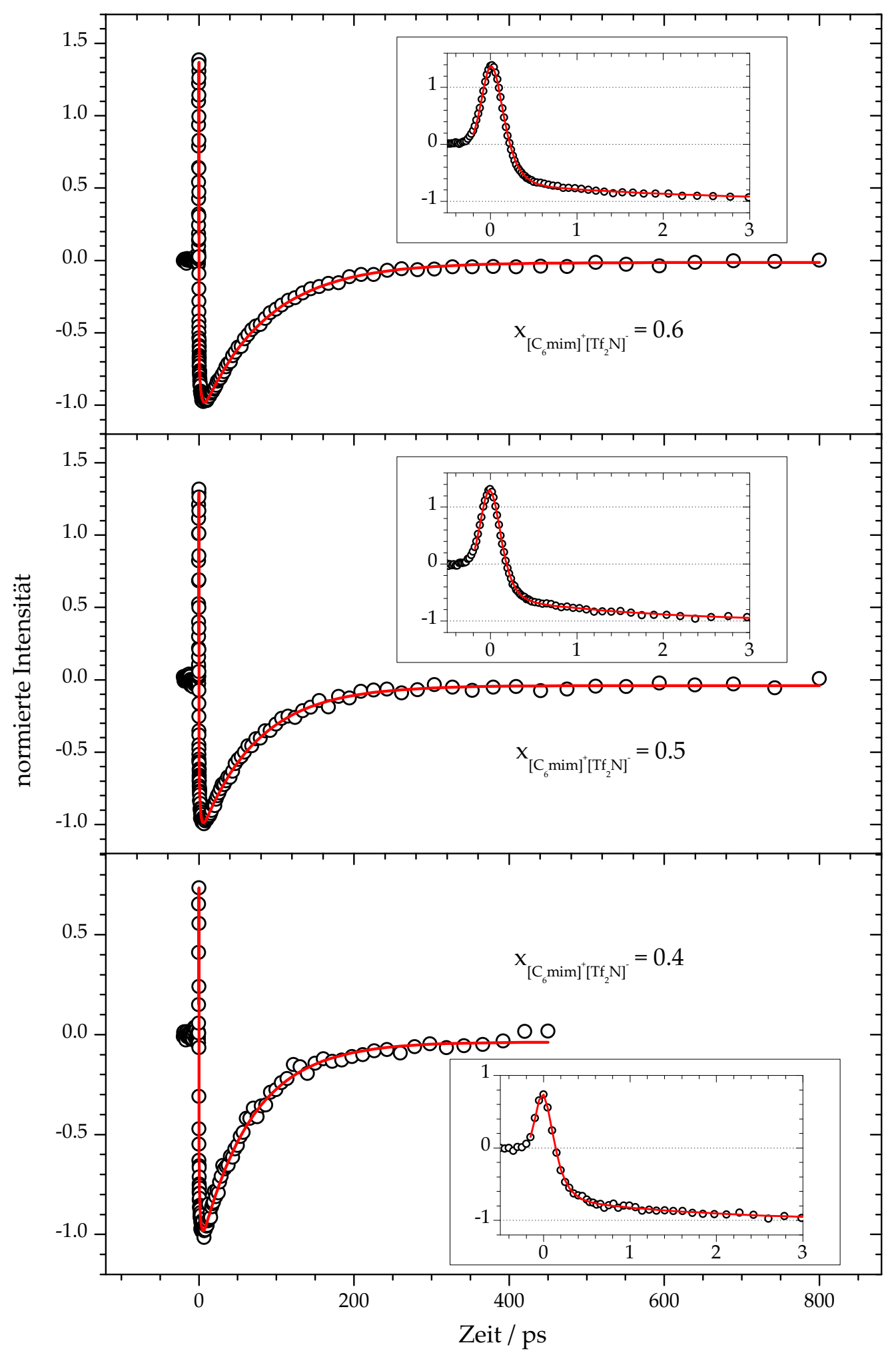

Abbildung 5.18: Wie Abbildung 5.17, aber in Mischungen mit der Zusammensetzung $\mathrm{x}_{I L}=0.6, \mathrm{x}_{I L}$ $=0.5$ und $\mathrm{x}_{I L}=0.4$. 


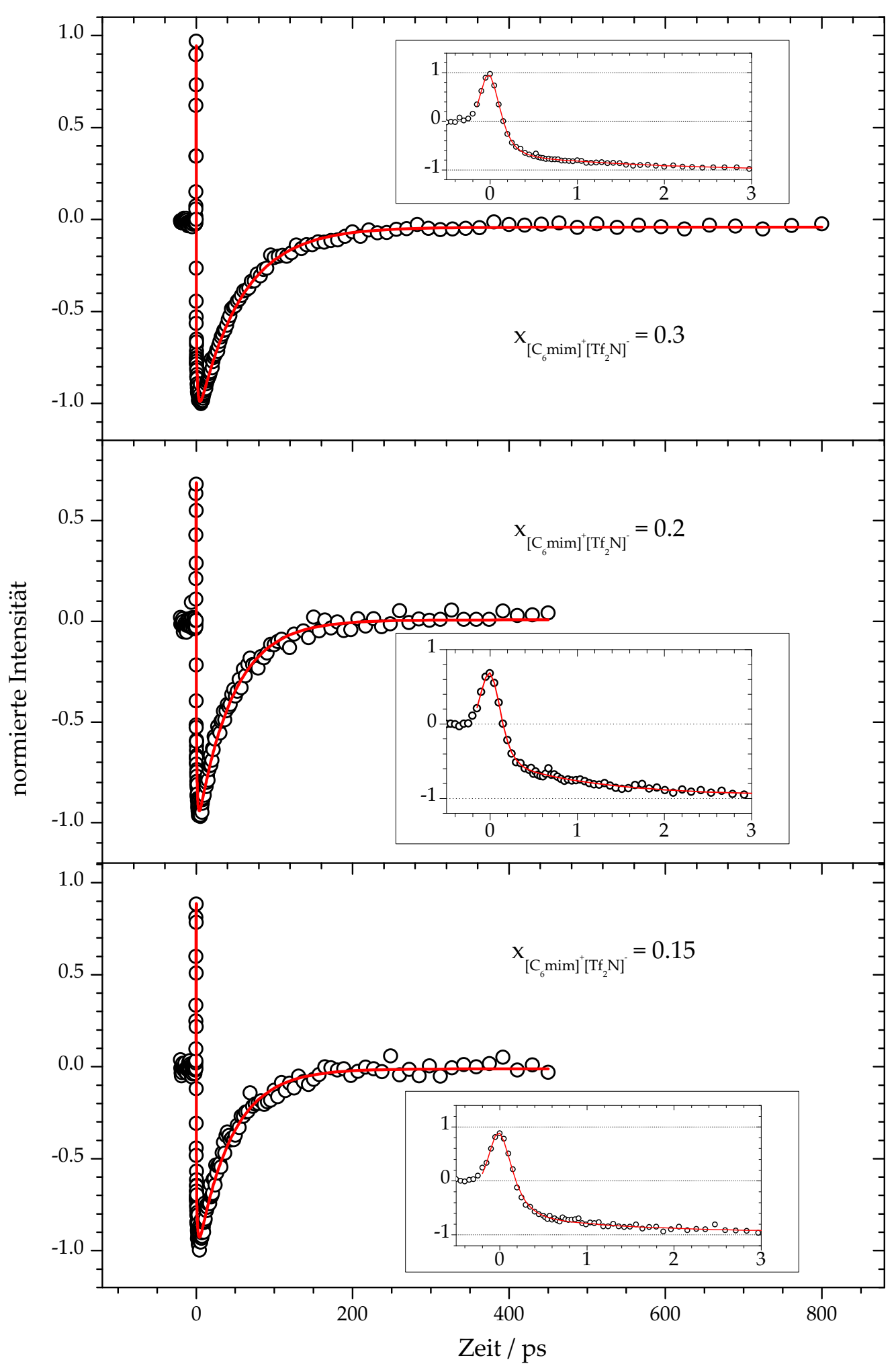

Abbildung 5.19: Wie Abbildung 5.17 aber in Mischungen mit der Zusammensetzung $\mathrm{x}_{I L}=0.3, \mathrm{x}_{I L}$ $=0.2$ und $x_{I L}=0.15$. 


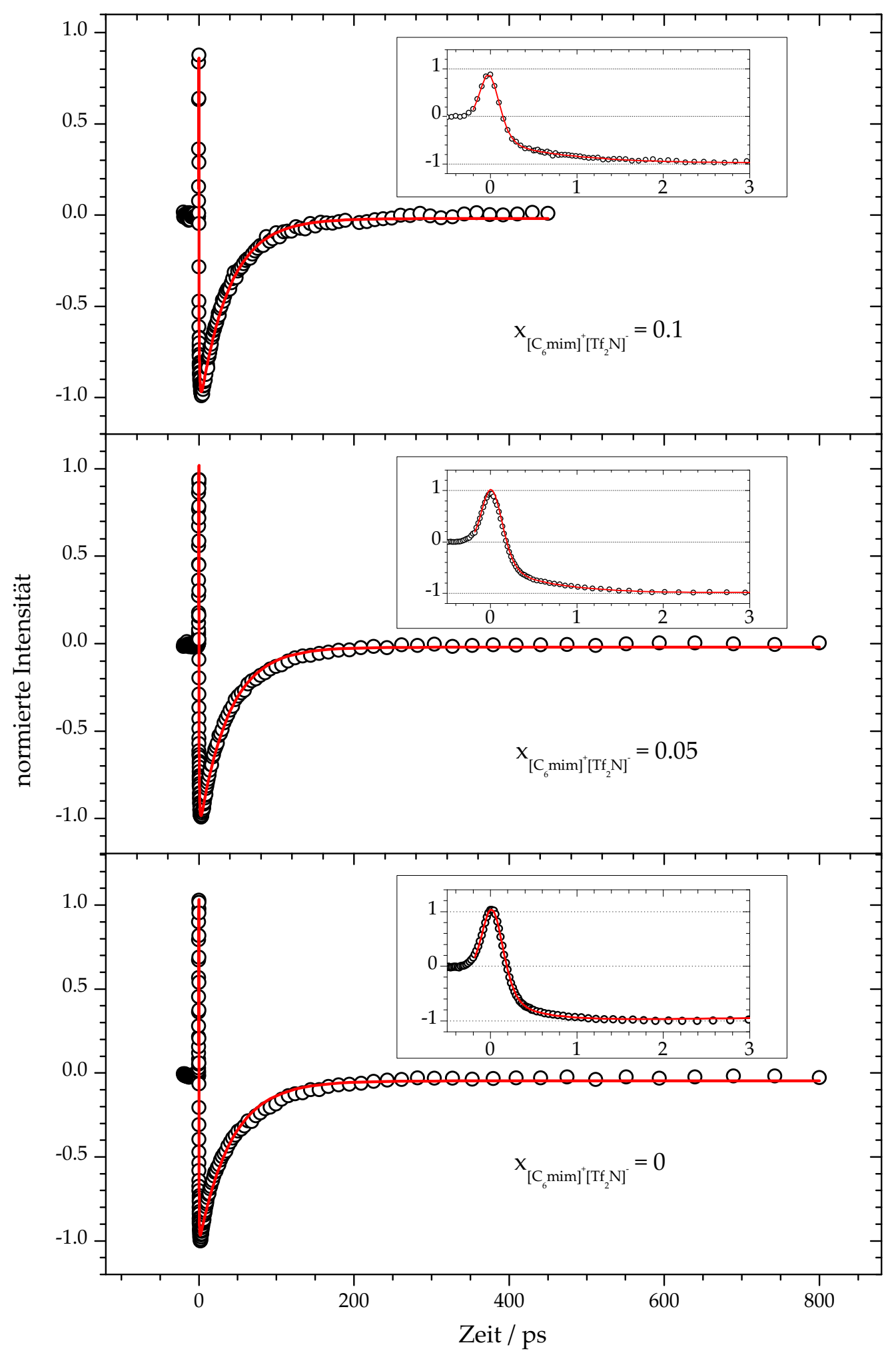

Abbildung 5.20: Wie Abbildung 5.17, aber für Mischungen mit der Zusammensetzung $\mathrm{x}_{I L}=0.1$, $\mathrm{x}_{I L}=0.05$ und $\mathrm{x}_{I L}=0$. 


\subsection{Ergebnisse für die Mischung $\left[\mathrm{C}_{2} \mathrm{mim}\right]^{+}\left[\mathrm{EtSO}_{4}\right]^{-} /$Acetonitril}

Die ionische Flüssigkeit $\left[\mathrm{C}_{2} \mathrm{mim}\right]^{+}\left[\mathrm{EtSO}_{4}\right]^{-}$ist für industrielle Anwendungen sehr vielversprechend, denn sie ist besonders preisgünstig und kann in hohen Maßstäben hergestellt werden. Im Vergleich mit anderen ionischen Flüssigkeiten hat sie eine ziemlich hohe Dielektrizitätskonstante (27.9). Außerdem verfügt sie über ein breites elektrochemisches Fenster und wird bereits als Reaktionsmedium und in Trennverfahren verwendet. ${ }^{118}$ Ein Nachteil bei dem Gebrauch von ionischen Flüssigkeiten ist deren hohe Viskosität. Hier könnte die Zugabe von molekularen Lösungsmitteln eine Abhilfe schaffen. So könnte die Viskosität herabgesetzt werden und die positiven Eigenschaften der ionischen Flüssigkeit noch erhalten bleiben. Für Anwendungen in der Synthese ist es von großem Interesse, die Wechselwirkungen von Edukten in diesem Medium kennen zu lernen, um eventuell Rekationen direkt steuern zu können.

In den folgenden Abschnitten werden die Untersuchungen der Wechselwirkungen von $12^{\prime} \mathrm{CA}$ in binären Mischungen von $\left[\mathrm{C}_{2} \mathrm{mim}\right]^{+}\left[\mathrm{EtSO}_{4}\right]^{-}$und Acetonitril vorgestellt. Zunächst wird in Abschnitt auf die Ergebnisse der transienten Absorptionsspektroskopie eingegangen. Es folgen die Untersuchungen mittels stationärer Absorptionsspektroskopie zur Charakterisierung der solvatochromen Verschiebung in den binären Mischungen. Eine Diskussion der Ergebnisse erfolgt in Abschnitt 5.4 .

\subsubsection{Ergebnisse des transienten Absorptionsexperiments}

Im Abschnitt 5.2.3 sind bereits Ergebnisse der Zweifarben-Transienten-Absorptionsspektroskopie von binären Mischungen einer ionischen Flüssigkeit vorgestellt worden. In diesem Abschnitt sollen die binären Mischungen von $\left[\mathrm{C}_{2} \mathrm{mim}\right]^{+}\left[\mathrm{EtSO}_{4}\right]^{-}$und Acetonitril beleuchtet werden. Als Indikator für die Wechselwirkungen in diesen Mischungen dient die molekulare Sonde 12 'CA. Von großem Interesse hierbei ist die Relaxation des $\mathrm{S}_{1} /$ ICTZustands, die durch das Experiment beobachtbar wird. Die energetische Lage dieses Zustands relativ zum Grundniveau hängt stark von der Zusammensetzung der Mischungen ab. Daher können durch die ermittelte Lebensdauer $\tau_{1}$ dieses Zustands Erkenntnisse über die mikroskopische Umgebung der Sonde gewonnen werden. Die gemessenen Signale sind in den Abbildungen 5.21 bis 5.23 dargestellt. Zusätzlich sind die kinetischen Modellierun- 
gen nach Gleichung 5.8 nebst einem Ausschnitt der Signale bei kurzen Zeiten enthalten. In Tabelle 5.3 sind die Anpassungsparamter angegeben. Das Signal für reines Acetonitril ist bereits in Abschnitt 5.2.3 Abbildung 5.20 dargestellt.

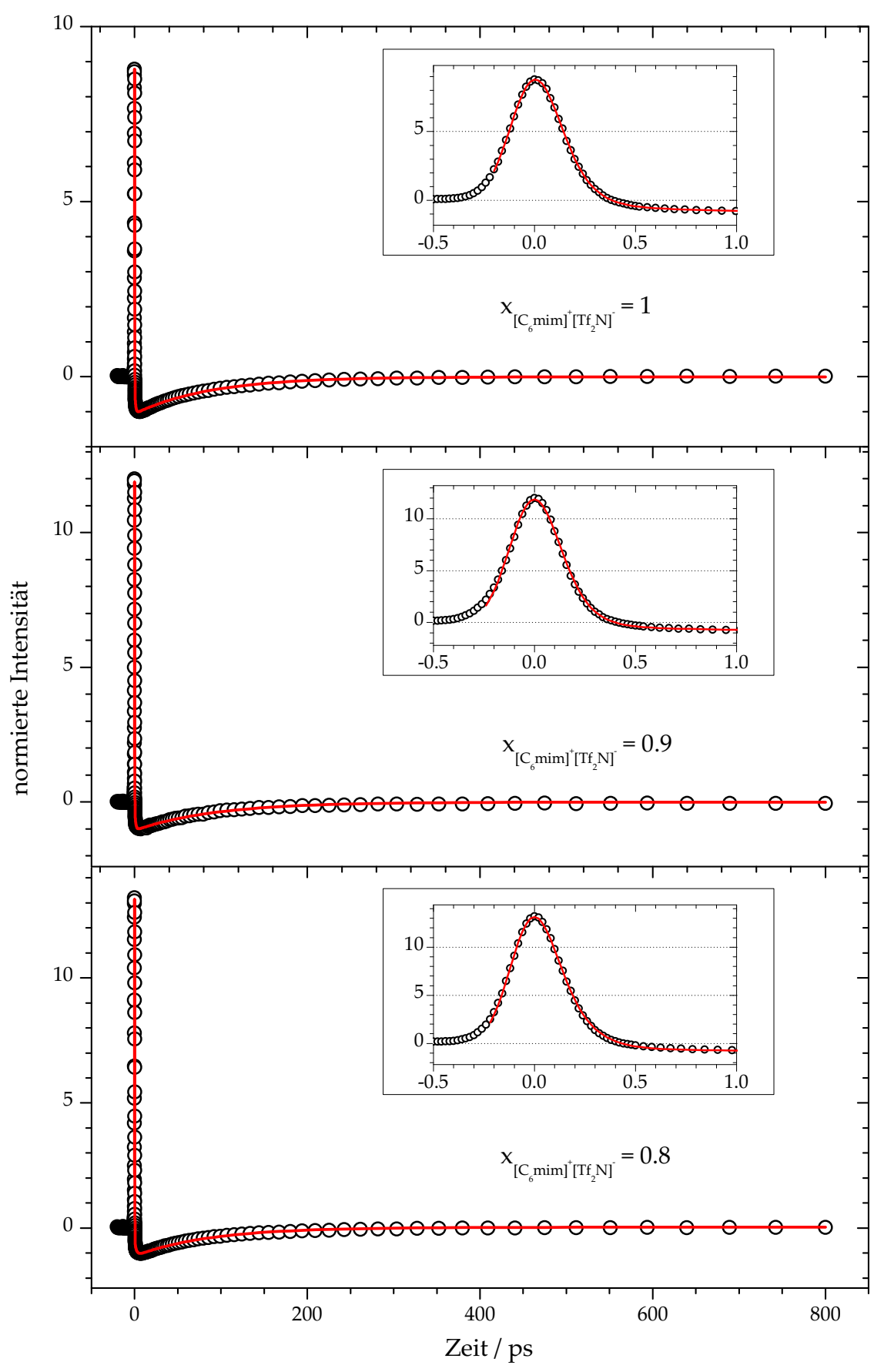

Abbildung 5.21: Transiente Absorptionssignale von $12^{\prime} \mathrm{CA}$ in binären Mischungen von $\left[\mathrm{C}_{2} \mathrm{mim}\right]^{+}\left[\mathrm{EtSO}_{4}\right]^{-}$und Acetonitril mit einer Zusammensetzung von $\mathrm{x}_{I L}=1, \mathrm{x}_{I L}=0.9$ und $\mathrm{x}_{I L}=0.8$ (Anregung: $430 \mathrm{~nm}$; Abfrage: $860 \mathrm{~nm}$ ). (一): kinetische Anpassung. 


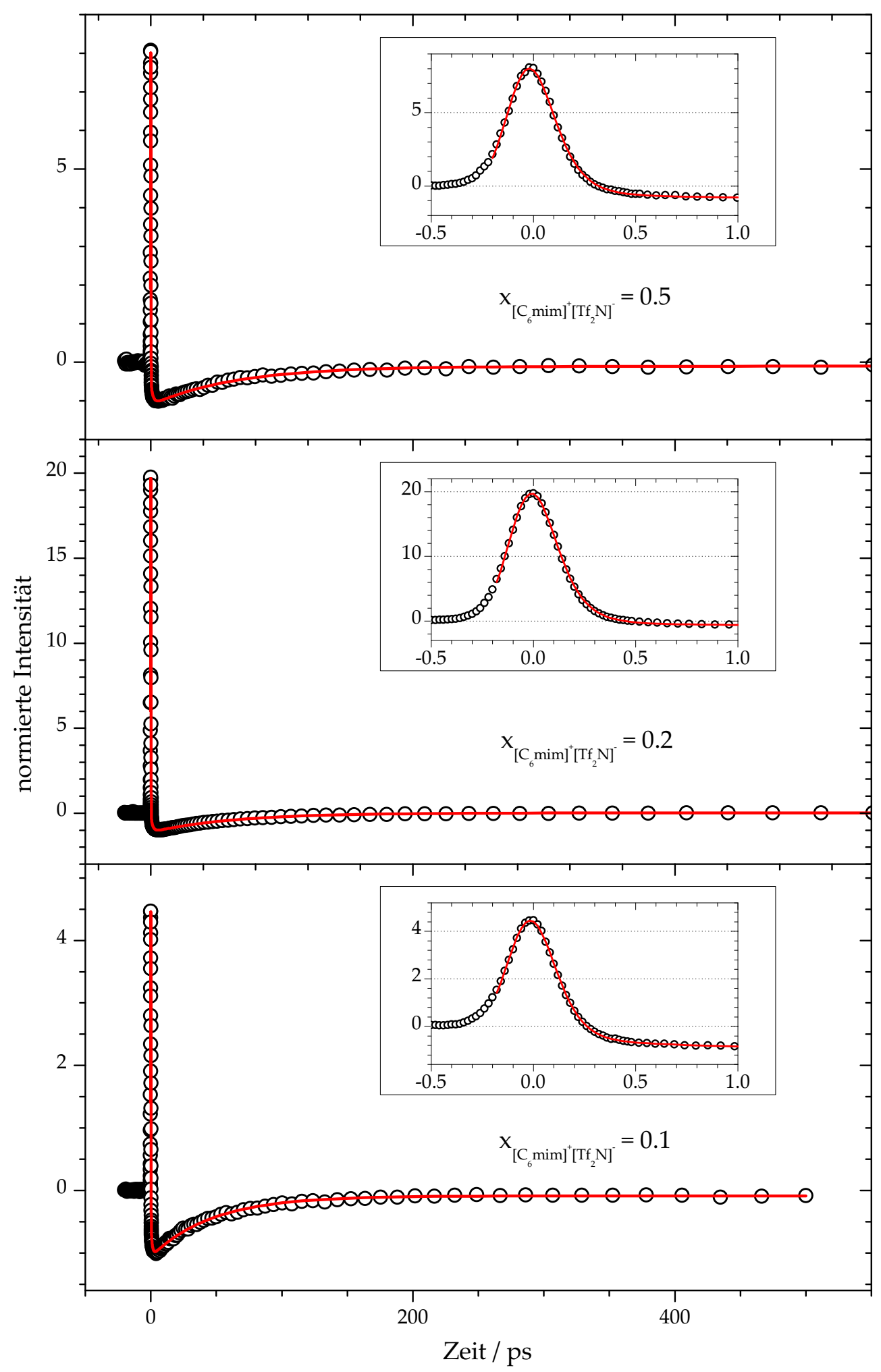

Abbildung 5.22: Wie Abbildung 5.21, aber für Mischungen mit einer Zusammensetzung von $\mathrm{x}_{I L}=$ $0.5, \mathrm{x}_{I L}=0.2$ und $\mathrm{x}_{I L}=0.1$. 


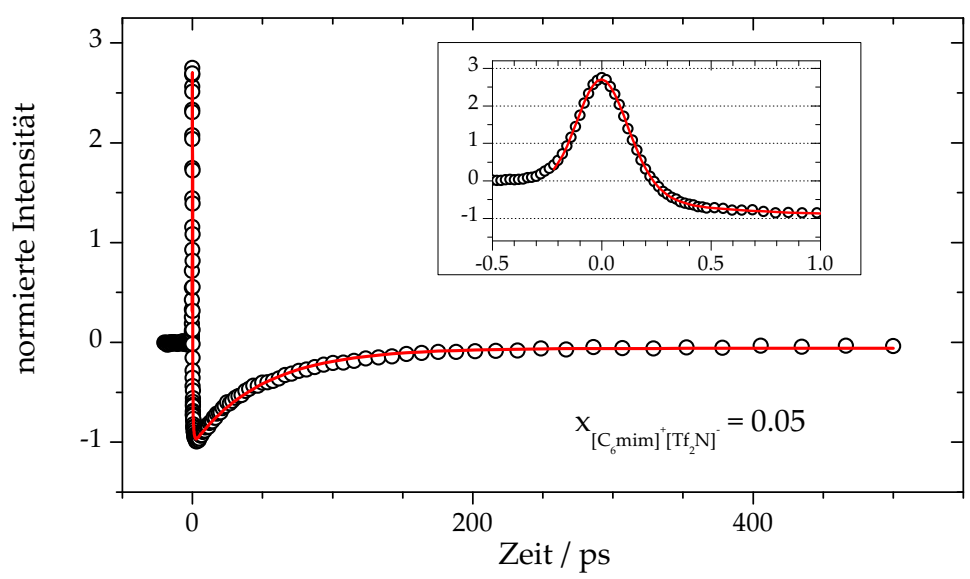

Abbildung 5.23: Wie Abbildung 5.21, aber für eine Mischung mit der Zusammensetzung $x_{I L}=0.05$.

Den Signalen ist ein schneller Anstieg zu entnehmen. Dieser wird durch die $S_{0} \rightarrow S_{2}$ Absorption hervorgerufen. Nach dieser Absorption kommt es zu einer schnellen inneren Konversion $S_{2} \rightarrow S_{1} /$ ICT. Diese läuft auf einer ultrakurzen Zeitskala von etwa $\tau_{2}=100 \mathrm{fs}$ ab. Der angeregte Zustand von 12'CA hat ein anders Dipolmoment als der Grundzustand, daher müssen sich die Ionen bzw. Moleküle in dem Feld neu ausrichten. Der $\mathrm{S}_{1}$ /ICT-Zustand wird mit steigendem Stoffmengenanteil der ionischen Flüssigkeit relativ zum Grundzustand stabilisiert. Diese erste Reorientierung der Ionen bzw. der Moleküle der binären Mischungen unter Bildung eines stabilisierten $\mathrm{S}_{1} /$ ICT-Zustands wird mit der Zeitkonstante $\tau_{3}$ wiedergegeben. Die Relaxation des $\mathrm{S}_{1} /$ ICT-Zustands zurück in den Grundzustand wird schließlich durch $\tau_{1}$ repräsentiert. Die Amplituden der verschiedenen Beiträge werden entsprechend mit $\mathrm{A}_{1}$ bis $\mathrm{A}_{3}$ symbolisiert, $\gamma$ ist die Pulslänge der Anregungspulse und $\mathrm{A}_{0}$ ist der Offset der Signale.

Tabelle 5.3: Anpassungsparameter

\begin{tabular}{clccccccccc}
\hline $\mathrm{Nr}$. & $\mathrm{x}_{I L}$ & $\mathrm{t}_{0}[\mathrm{ps}]$ & $\gamma[\mathrm{ps}]$ & $\mathrm{A}_{1}$ & $\tau_{1}$ [ps] & $\mathrm{A}_{2}$ & $\tau_{2}[\mathrm{ps}]$ & $\mathrm{A}_{3}$ & $\tau_{3}[\mathrm{ps}]$ & $\mathrm{A}_{0}$ \\
\hline 1 & 0 & -0.03 & 0.1 & -0.5 & 42 & 5.9 & 0.1 & 0.3 & 0.4 & -0.05 \\
2 & 0.05 & -0.05 & 0.1 & -0.5 & 50 & 16.4 & 0.1 & 0.3 & 0.7 & -0.06 \\
3 & 0.1 & -0.07 & 0.1 & -0.5 & 46 & 20.7 & 0.1 & 0.3 & 0.9 & -0.09 \\
4 & 0.2 & -0.07 & 0.1 & -0.6 & 62 & 60.7 & 0.1 & 0.5 & 1.5 & 0.02 \\
5 & 0.5 & -0.08 & 0.1 & -0.5 & 65 & 23.5 & 0.1 & 0.3 & 1.8 & -0.10 \\
6 & 0.8 & -0.07 & 0.1 & -0.6 & 87 & 32.2 & 0.1 & 0.3 & 2.0 & 0.03 \\
7 & 0.9 & -0.06 & 0.1 & -0.5 & 93 & 56.7 & 0.1 & 0.5 & 1.0 & -0.01 \\
8 & 1.0 & -0.06 & 0.1 & -0.5 & 87 & 35.8 & 0.1 & 0.4 & 1.0 & -0.01 \\
\hline
\end{tabular}




\subsubsection{Solvatochrome Untersuchungen}

Die solvatochrome Verschiebung von $12^{\prime} \mathrm{CA}$ in binären Mischungen von $\left[\mathrm{C}_{2} \mathrm{mim}\right]^{+}\left[\mathrm{EtSO}_{4}\right]^{-}$ und Acetonitril zeigt die gleiche Tendenz, wie in Abschnitt 5.2.1 gezeigt. Mit zunehmenden Stoffmengenanteil der ionischen Flüssigkeit kommt es zu einer Rotverschiebung, wie in Abbildung $5.24 \mathrm{zu}$ sehen. Hier sind die Banden der $S_{0} \rightarrow S_{2}$-Absorption stark vergrößert dargestellt. Die Abhängigkeit des Absorptionsmaximums von der Zusammensetzung ist Abbildung $5.25 \mathrm{zu}$ entnehmen. Alleine dieses Ergebnis vor Augen würde vermuten lassen, dass es eine Art präferierte Solvatation gibt, da es zu Beginn keine wesentliche Änderung des Absorptionsmaximums nach Zugabe von Acetonitril gibt. Dieser Befund muss daher eingehend in der Dikussion analysiert werden.

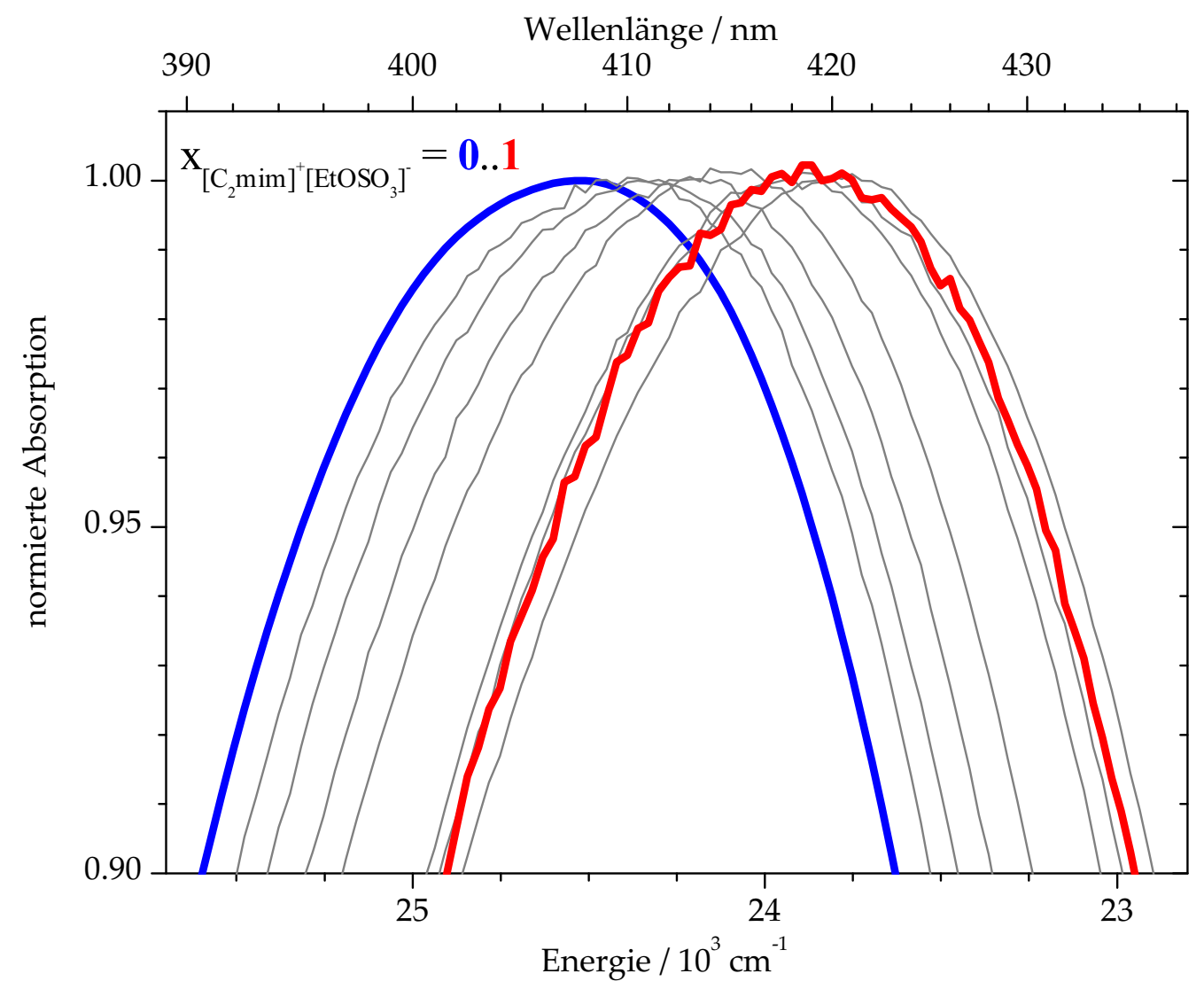

Abbildung 5.24: Stationäre Absorptionsspektren von 12'CA in binären Mischungen von $\left[\mathrm{C}_{2} \mathrm{mim}\right]^{+}\left[\mathrm{EtSO}_{4}\right]^{-}$und Acetonitril bei $298 \mathrm{~K}$. 


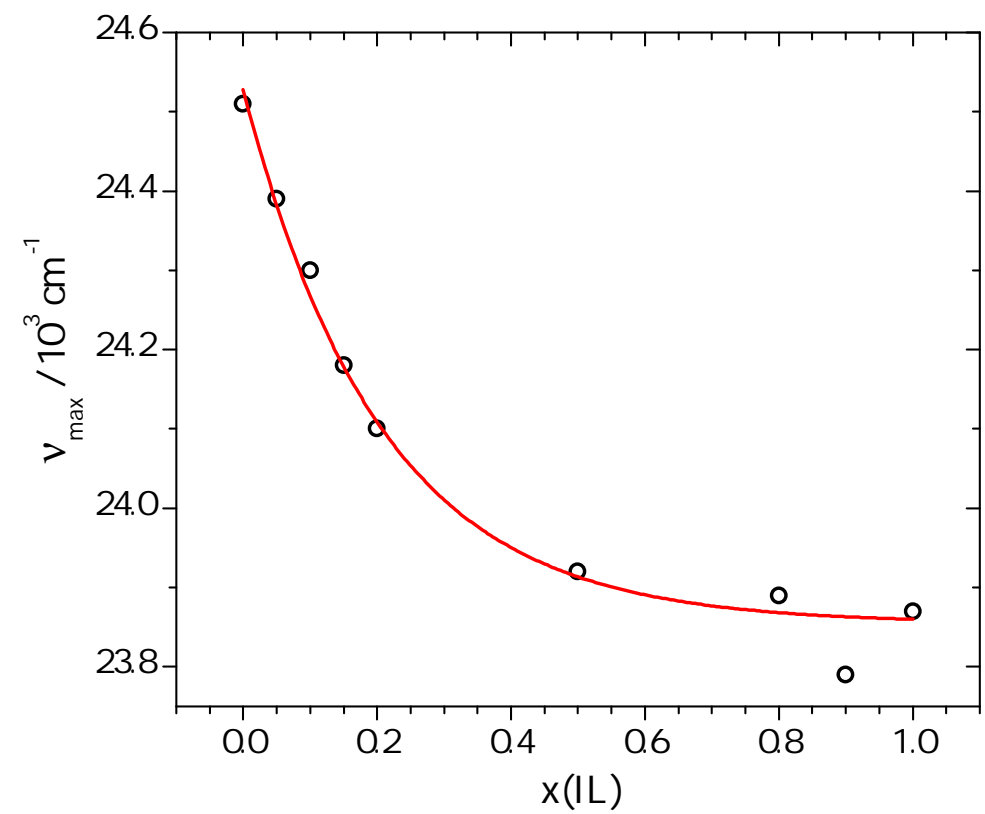

Abbildung 5.25: Abhängigkeit des stationären Absorptionsmaximums der Sonde $12^{\prime} \mathrm{CA}$ von der Zusammensetzung in binären Mischungen von $\left[\mathrm{C}_{2} \mathrm{mim}\right]^{+}\left[\mathrm{EtSO}_{4}\right]^{-}$und Acetonitril.

\subsection{Diskussion}

Neben den reinen ionischen Flüssigkeiten stellen deren binäre Mischungen mit organischen Lösungsmitteln interessante Medien dar. Sie vereinen in gewisser Weise die Eigenschaften beider Komponenten und eröffnen einen anderen Blickwinkel auf das Studium der ionischen Flüssigkeiten. Die Wechselwirkung der molekularen Sonde 12'CA mit diesen Medien ist mit Hilfe von transienten und stationären Absorptionsmethoden eingehend untersucht worden. Die Ergebnisse sind in Kapitel 5.2 und 5.3 dargestellt worden und werden in diesem Abschnitt ausführlich diskutiert.

Die stationären Absorptionsspektren der einzelnen binären Mischungen werden im Hinblick auf eine solvatochromen Verschiebung analysiert. Hierbei zeigt sich sowohl bei $\left[\mathrm{C}_{6} \mathrm{~m}-\right.$ $\mathrm{im}]^{+}\left[\mathrm{Tf}_{2} \mathrm{~N}\right]^{-}$als auch bei $\left[\mathrm{C}_{2} \mathrm{mim}\right]^{+}\left[\mathrm{EtSO}_{4}\right]^{-}$eine Verschiebung zu kleineren Wellenlängen mit der Zugabe von Acetonitril. Dies kann den Abbildungen 5.2 und 5.24 entnommen werden. In beiden Fällen gibt es einen nicht-linearen Zusammenhang zwischen dem Absorptionsmaximum und der Zusammensetzung der binären Mischung. Erst nach Zugabe von etwa 0.5 bis 0.6 Mol-\% Acetonitril kommt es zu einer stärkeren Verschiebung in den kürzerwelligen Bereich. Kann man daher davon ausgehen, dass es eine präferierte Löslichkeit von 
12 'CA in der ionischen Flüssigkeit gibt? Bei näherer Betrachtung ist dies auszuschließen. Die Moleküle der ionischen Flüssigkeit sind wesentlich größer als Acetonitril und haben eine höhere Masse und damit auch eine höhere Polarisierbarkeit. Bei der solvatochromen Verschiebung kommt es daher zu einem Masseneffekt. Wird das Absorptionsmaximum von $12^{\prime} \mathrm{CA}$ in den binären Mischungen als Funktion des Massenanteils der jeweiligen ionischen Flüssigkeit aufgetragen, ergibt sich ein annähernd linearer Verlauf. Dies wird in Abbildung 5.26 deutlich.

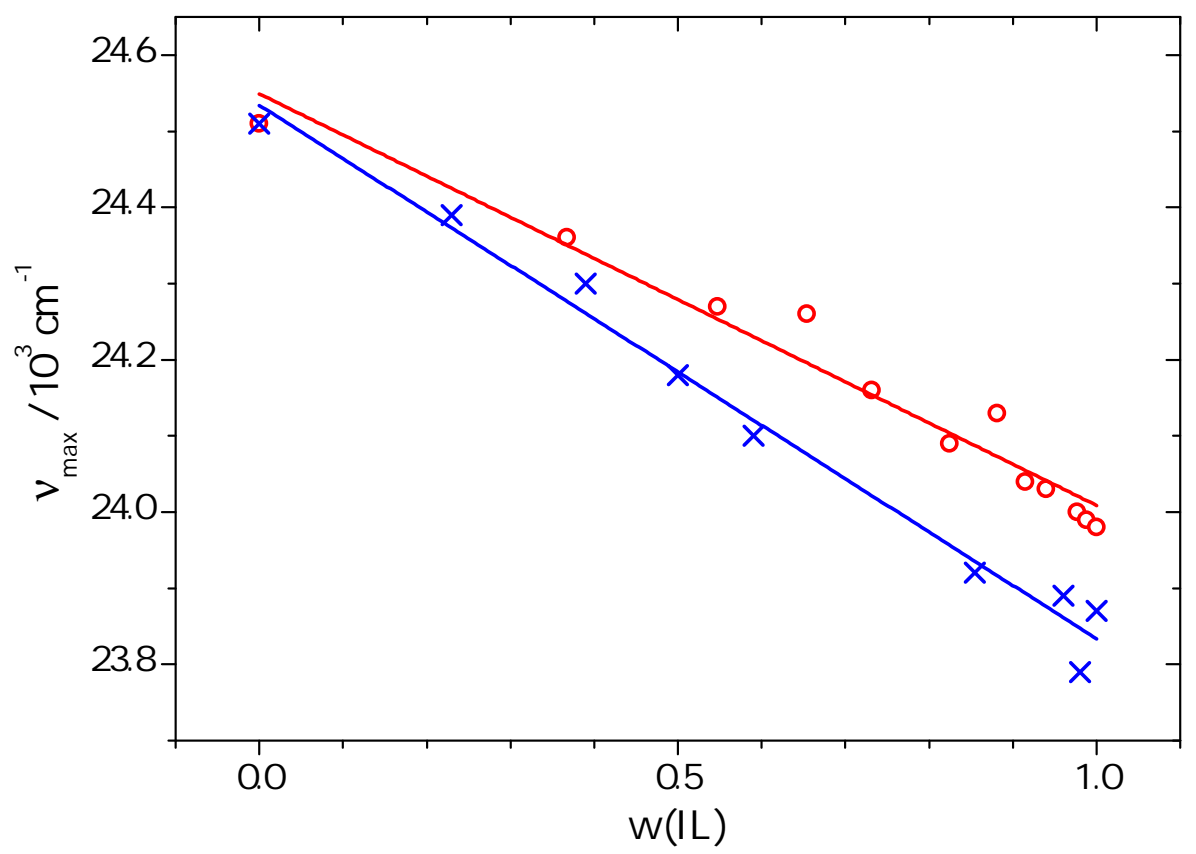

Abbildung 5.26: Absorptionsmaxima von $12^{\prime} \mathrm{CA}$ als Funktion des Massenanteils der ionischen Flüssigkeiten $\left[\mathrm{C}_{6} \mathrm{mim}\right]^{+}\left[\mathrm{Tf}_{2} \mathrm{~N}\right]^{-}(\circ)$ und $\left[\mathrm{C}_{2} \mathrm{mim}\right]^{+}\left[\mathrm{EtSO}_{4}\right]^{-}(\times)$.

Bei der reinen Betrachtung des Absorptionsmaximums in Abhängigkeit vom Stoffmengenanteil wird die Solvenspolarisierbarkeit ausgeklammert. Nach Gleichung 5.9 kann die Polarisierbarkeit anhand des Brechungsindexes $n$ quantifiziert werden. Der sich durch Zugabe von Acetonitril ändernde Brechungsindex hat natürlich einen erheblichen Einfluss auf das Absoprtionsverhalten der molekularen Sonde. Trägt man das Absorptionsmaximum in Abhängigkeit von der Lorentz-Lorenz-Funktion auf, ergibt sich ein linearer Verlauf der solvatochromen Verschiebung. Anhand dieses Befundes kann eine präferierte Solvatation weitestgehend ausgeschlossen werden. In der nachfolgenden Abbildung wird dieser Zusammenhang ersichtlich. Für diese Korrelation sind zunächst die Brechungsindizes der bi- 
nären Mischungen unterschiedlicher Zusammensetzung gemessen worden, da diese nicht in der Literatur vorhanden sind. Eine Zusammenfassung aller Brechungsindizes steht in Tabelle 5.4 am Ende dieser Diskussion in der nochmals alle experimentell ermittelten Größen der Mischungen beider ionischen Flüssigkeiten zu finden sind.

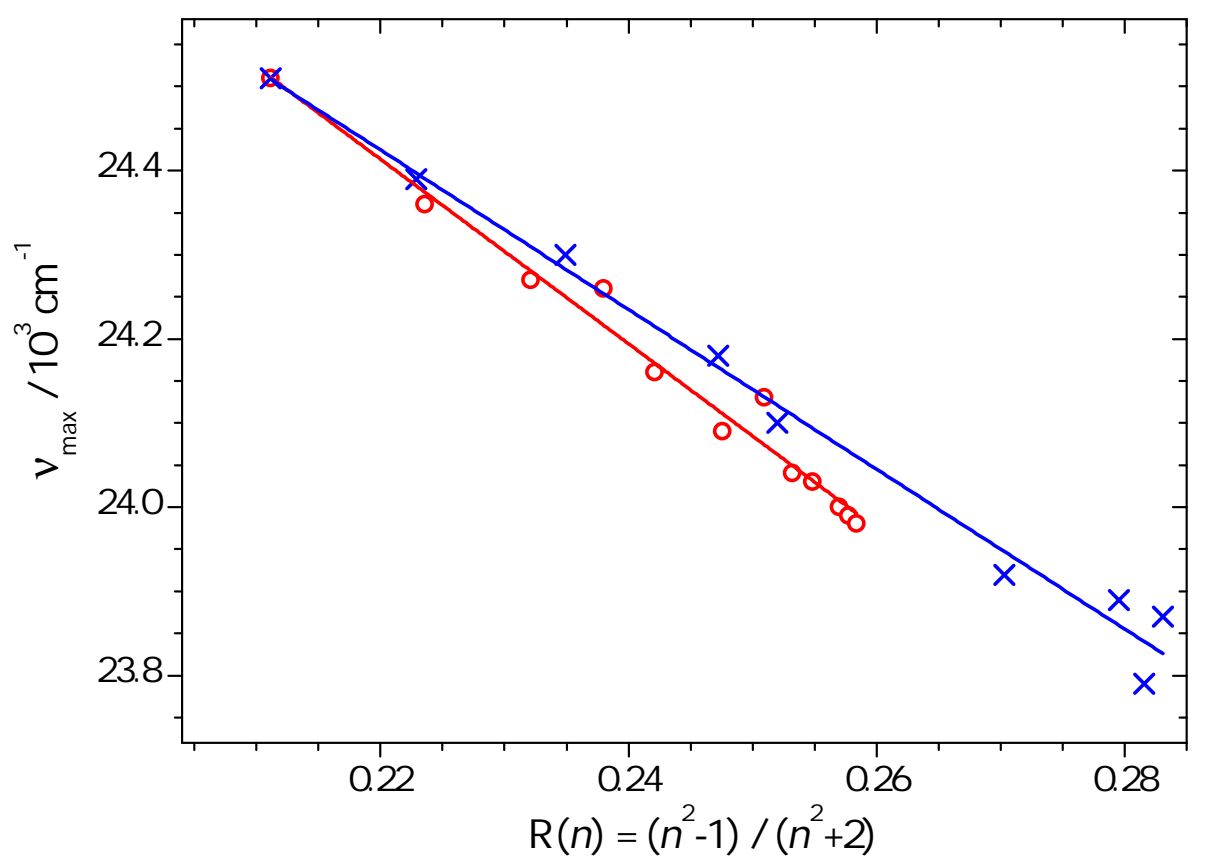

Abbildung 5.27: Abhängigkeit des Absorptionsmaximums von 12' $\mathrm{CA}$ in den binären Mischungen von $\left[\mathrm{C}_{6} \mathrm{mim}\right]^{+}\left[\mathrm{Tf}_{2} \mathrm{~N}\right]^{-}(\circ)$ und $\left[\mathrm{C}_{2} \mathrm{mim}\right]^{+}\left[\mathrm{EtSO}_{4}\right]^{-}(\times)$von der Polarisierbarkeit.

Die ultraschnelle Dynamik von $12^{\prime} \mathrm{CA}$ in reinen ionischen Flüssigkeiten ist bereits mittels zeitaufgelöster transienter Absorptionsspektroskopie untersucht worden. ${ }^{33}$ Ergebnisse für die Wechselwirkung dieser Sonde in reinen molekularen Lösungsmitteln sind ebenfalls in der Literatur zu finden. 81 Bei diesen Messungen konnte gezeigt werden, dass dieses carbonylsubstituierte Apocarotinoid einen niedrig liegenden elektronisch angeregten Zustand mit intramolekularem Charge-Transfer Charakter hat. Dieser Zustand wird daher als $\mathrm{S}_{1} / \mathrm{ICT}$ bezeichnet. Die Änderung der relativen Stabilisierung dieses Zustands im Vergleich mit dem Grundzustand und damit die Lebensdauer geht mit der Polarität des Lösungsmittels einher. In dieser Arbeit sind zum ersten mal transiente Absorptionsexperimente von einem Carotinoid in binären Mischungen von ionischen Flüssigkeiten mit organischen Lösungsmitteln durchgeführt worden. Durch diese Experimente konnten die Lebensdauern des $\mathrm{S}_{1}$ /ICT-Zustands in Mischungen verschiedener Zusammensetzungen 
bestimmt werden. Diese Messungen sind im nahen IR-Bereich durchgeführt worden. Im Fall von $\left[\mathrm{C}_{6} \mathrm{mim}\right]^{+}\left[\mathrm{Tf}_{2} \mathrm{~N}\right]^{-}$sind die Lebensdauern zusätzlich durch eine globale Analyse in einem breiten Spektrum von 350 bis $770 \mathrm{~nm}$ bestimmt worden. Betrachtet man die Lebensdauern, die durch das Zweifarben-Pump-Probe Experiment bestimmt worden sind, als Funktion der Zusammensetzung der binären Mischungen, dann ergibt sich folgendes Bild.

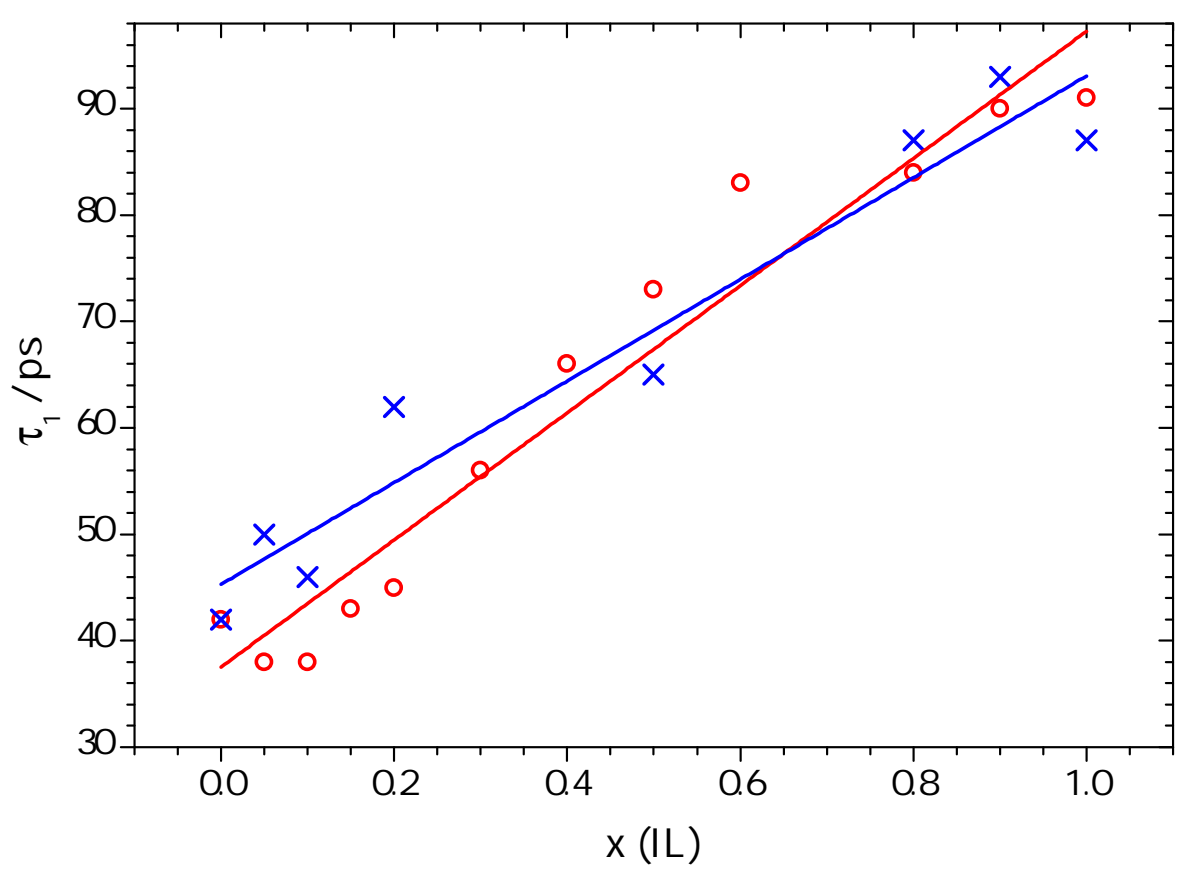

Abbildung 5.28: Abhängigkeit der Lebensdauer $\tau_{1}$ von der Zusammensetzung. $\left[\mathrm{C}_{6} \mathrm{mim}^{+}\left[\mathrm{Tf}_{2} \mathrm{~N}\right]^{-}\right.$ (०) und $\left[\mathrm{C}_{2} \mathrm{mim}\right]^{+}\left[\mathrm{EtSO}_{4}\right]^{-}(\times)$.

Der Anstieg der Lebensdauer mit steigendem Stoffmengenanteil der ionischen Flüssigkeit scheint weitestgehend linear zu sein. Die einzelnen Werte streuen ein wenig, doch die Tendenz ist klar erkenntbar. Der Fehler der bestimmten Lebensdauern liegt zwischen 5 und 10\% und wird von der Ungenauigkeit der Anpassung sowie die Ungenauigkeit beim Herstellen der Mischungen maßgeblich beeinflusst.

Neben der Lebensdauer $\tau_{1}$ können zwei weitere Lebensdauern extrahiert werden. Für die schnelle innere Konversion $S_{2} \rightarrow S_{1} /$ ICT wird eine Lebensdauer $\tau_{2}$ von etwa 100 fs gefunden. Diese Lebensdauer liegt an der unteren Grenze der zeitlichen Auflösung des Experiments, scheint aber unabhängig von der Art des Lösungsmittels zu sein. Eine weitere Zeitkonstante wird bei der Bildung des $\mathrm{S}_{1}$ /ICT-Zustands erkennbar. Diese Zeitkonstan- 
te $\tau_{3}$ ist in reinem Acetonitril kleiner als in den gemessenen ionischen Flüssigkeiten. Die Solvensrelaxation spielt eine wichtige Rolle bei dieser Zeitkonstante. Bis zu einer hohen Verdünnung der ionischen Flüssigkeit von $\mathrm{x}_{I L}=0.4$ bis 0.2 bleibt $\tau_{3}$ etwa konstant. Erst bei diesen hohen Verdünnungen kommt es zu einer starken Abnahme von $\tau_{3}$. Dies kann in Abbildung 5.29 nachvollzogen werden.

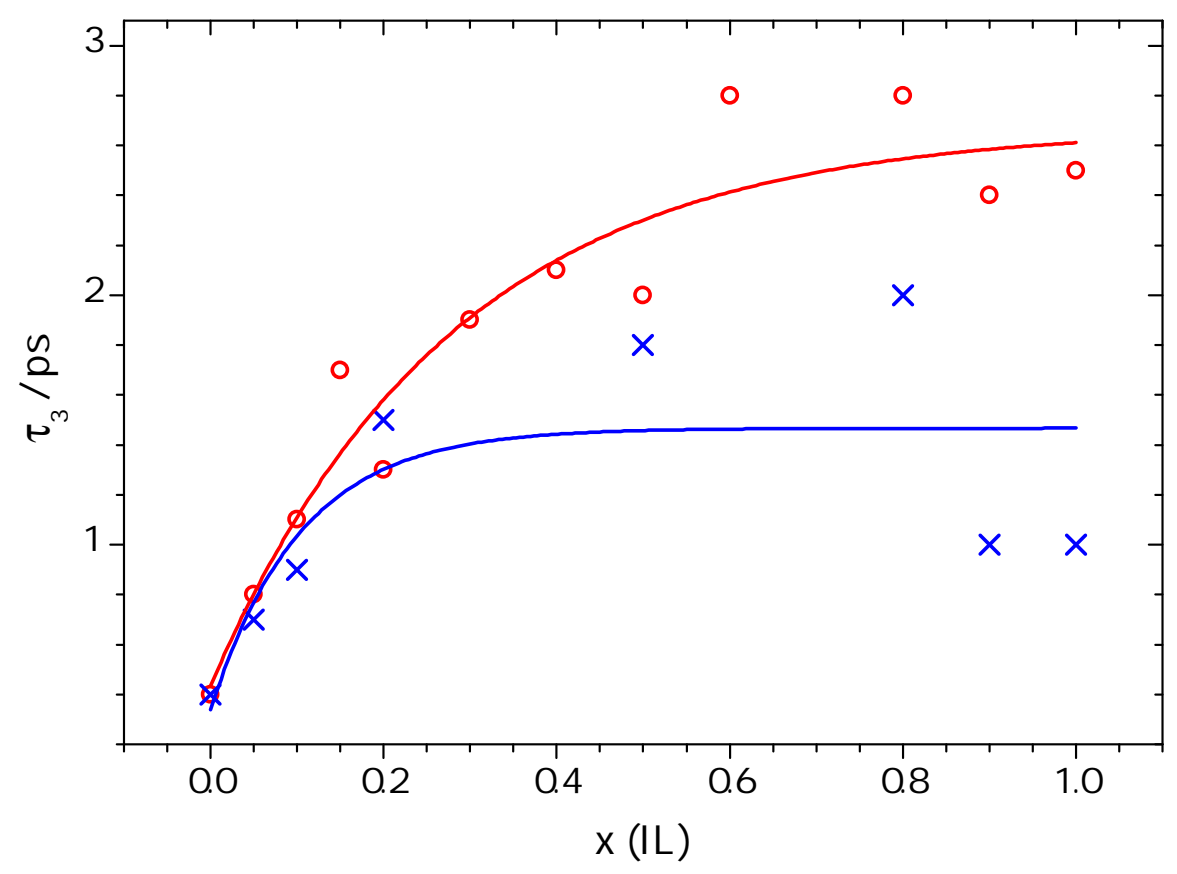

Abbildung 5.29: Abhängigkeit der Lebensdauer $\tau_{3}$ von der Zusammensetzung. $\left[\mathrm{C}_{6} \operatorname{mim}\right]^{+}\left[\mathrm{Tf}_{2} \mathrm{~N}\right]^{-}$ (०) und $\left[\mathrm{C}_{2} \mathrm{mim}\right]^{+}\left[\mathrm{EtSO}_{4}\right]^{-}(\times)$.

In früheren Arbeiten zum Thema Dynamik von 12'CA in organischen Lösungsmitteln ist zu erkennen, dass es eine Polaritätsskala gibt, die die Lebensdauer des $\mathrm{S}_{1} /$ ICT-Zustands mit der Polaritätsfunktion $\Delta f$ korreliert. ${ }^{81}$ Die Polaritätsfunktion von Lösungsmitteln ist folgendermaßen definiert:

$$
\Delta f=\mathrm{R}(\epsilon)-\mathrm{R}(n)=\frac{\epsilon-1}{\epsilon+2}-\frac{n^{2}-1}{n^{2}+2} .
$$

Es handelt sich also um eine Funktion der Dielektrizitäskonstanten $\epsilon$ und des Brechungsindex $n$. Die Polarisierbarkeit der Moleküle wird durch den ersten Teil $\mathrm{R}(\epsilon)$ der Gleichung wiedergegeben und der zweite Term $\mathrm{R}(n)$ steht für die hochfrequente Polarisierbarkeit der Elektronen. Die Differenz der beiden Teile kann also als Maßstab für die Polarität der Lösungsmittel stehen. Für die molekulare Sonde 12'CA sind die $\tau_{1}$-Werte im Bereich der 
nichtpolaren bis mittelpolaren Lösungsmittel kaum von der Polarität abhängig. Im Bereich der polaren Lösungsmittel ist allerdings eine starke Abnahme zu verzeichnen. Diese Polaritätsskala ist in Abbildung 5.30 gezeigt. Die Abnahme der Lebensdauer mit steigender Polarität des Lösungsmittels kann auf Basis des „Energy-Gap-Laws“ als Erniedrigung der

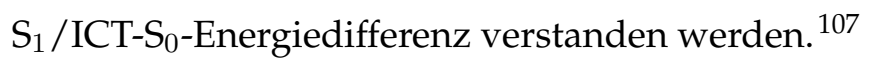

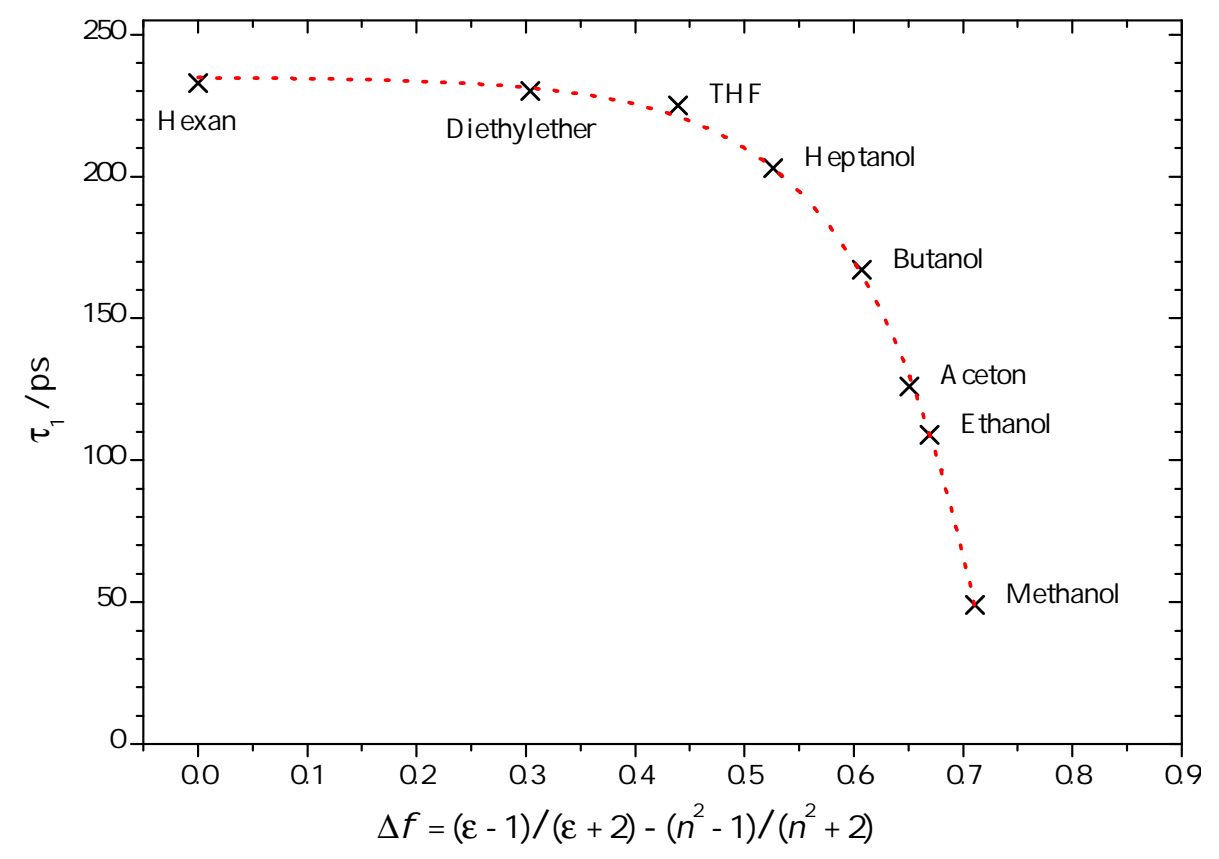

Abbildung 5.30: Polaritätsskala von $12^{\prime} \mathrm{CA}$ in organischen Lösungsmitteln.

Auf dieser Polaritätsskala befinden sich die für diese Arbeit relevanten ionischen Flüssigkeiten im Bereich zwischen Ethanol und Methanol. Die Lebensdauer $\tau_{1}$ von 12 'CA liegt für viele ionische Flüssigkeiten trotz ihrer unterschiedlichen chemischen Struktur und Ionenzusammensetzung in einem eng definierten Bereich. $\cdot 33$ Es wird angenommen, dass die Stabilisierung des $\mathrm{S}_{1}$ / ICT-Zustands in allen ionischen Flüssigkeiten durch ähnliche Wechselwirkungen hervorgerufen wird. Hierbei kommt es wahrscheinlich zu einer elektrostatischen Wechselwirkung zwischen der positiven Ladung der Kationen mit dem negativ geladenen Carbonylsauerstoff der molekularen Sonde. Die $\tau_{1}$-Werte des Carotinoids sind in den ionischen Flüssigkeiten kleiner als das Verhalten dieses Moleküls in den organischen Lösungsmitteln im gleichen $\Delta f$-Bereich vermuten lässt. Da die Polaritätsfunktion (Gleichung 5.10) auf der statischen Dielektrizitätskonstante und dem Brechungsindex des Lösungsmittels gründet, kann die Abweichung von der Skala auf eine wesentlich polare- 
re mikroskopische Umgebung in den ionischen Flüssigkeiten zurückgeführt werden. Dies könnte eine polarere Umgebung sein, als die makroskopischen Größen $\epsilon$ und $n$ vermuten lassen. Ähnliche Abweichungen berichtet MARONCELLI für die Energie der Lösungsmittelreorganisation von Coumarin 153 in ionischen Flüssigkeiten. Die Korrelation dieser Energie mit $\mathrm{R}(\epsilon)$ weicht von dem Verhalten in organischen Lösungmitteln ab. ${ }^{124}$ Interessant ist es, zu sehen, wie sich die molekulare Sonde in binären Mischungen beider Arten von Lösungsmitteln verhält.

Die $\tau_{1}$-Werte von $12^{\prime} \mathrm{CA}$ in den Mischungen können empirisch mit einer „effektiven lokalen Dielektrizitätskonstanten“ korreliert werden, um der höheren lokalen Polarität Rechnung zu tragen. Hierbei nimmt man den Term $\mathrm{R}(\epsilon)$ als 1 an und erhält eine effektive Polaritätsfunktion $\Delta f_{e f f} \approx 1-\mathrm{R}(n)$. Eine Korrelation der $\tau_{1}$-Werte mit dieser Polaritätsfunktion ergibt folgendes Bild.

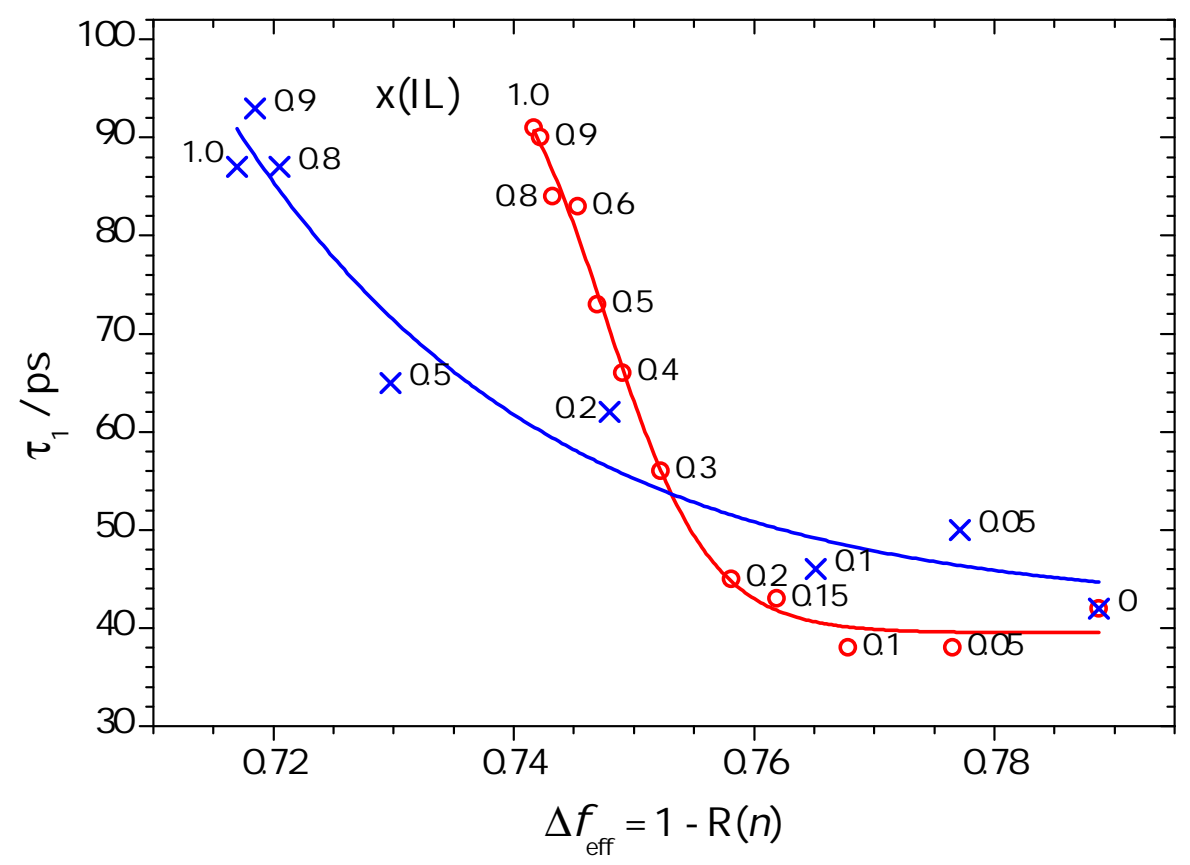

Abbildung 5.31: Polaritätsskala von $12^{\prime} \mathrm{CA}$ in binären Mischungen von $\left[\mathrm{C}_{6} \mathrm{mim}\right]^{+}\left[\mathrm{Tf}_{2} \mathrm{~N}\right]^{-}(\circ)$ und $\left[\mathrm{C}_{2} \mathrm{mim}\right]^{+}\left[\mathrm{EtSO}_{4}\right]^{-}(\times)$mit Acetonitril.

Die mikroskopische Umgebung von $12^{\prime} \mathrm{CA}$ in den binären Mischungen von $\left[\mathrm{C}_{6} \mathrm{mim}\right]^{+}$ $\left[\mathrm{Tf}_{2} \mathrm{~N}\right]^{-}$und $\left[\mathrm{C}_{2} \mathrm{mim}\right]^{+}\left[\mathrm{EtSO}_{4}\right]^{-}$mit Acetonitril scheint sich abgesehen von den verschiedenen Kationen zu unterscheiden. Wie in Abbildung 5.31 ersichtlich, ändert sich die lokale Polarität in den binären Mischungen von $\left[\mathrm{C}_{6} \mathrm{mim}\right]^{+}\left[\mathrm{Tf}_{2} \mathrm{~N}\right]^{-}$bis zu einer relativ hohen 
Verdünnung kaum. Die lokale Polarität wird in dieser Diskussion durch die effektive Polaritätsfunktion $\Delta f_{\text {eff }}$ ausgedrückt. Je höher die Werte dieser Funktion, desto größer ist die mikroskopische Polarität in der binären Mischung. Die molekulare Sonde 12'CA scheint bis zu einem Stoffmengenanteil von 80 \% des Acetonitrils in den Mischungen eine ähnlich polare Umgebung zu haben. In einem engen Polaritätsbereich von $\Delta f_{\text {eff }}=0.741$ bis 0.756 nimmt die Lebensdauer $\tau_{1}$ stark ab. Dies bedeutet, dass sich bei kaum ändernder Polarität die mikroskopische Umgebung der Sonde ändern muss. Vorstellbar ist ein erhalten bleibendes Ionennetzwerk der ionischen Flüssigkeit in der Umgebung der Sonde bis hin zu einer hohen Verdünnung von $\mathrm{x}_{I L}=0.2$. Dadurch bleibt die Wechselwirkung zwischen der Sonde und dem Kation der ionischen Flüssigkeit dominant. Allerdings könnten immer mehr Acetonitrilmoleküle in dieses Netzwerk eindringen um für eine zusätzliche relative Stabilisierung des $S_{1}$ /ICT-Zustands zu sorgen, ohne eine deutliche Änderung der mikroskopischen Polarität hervorzurufen. Erst bei höheren Verdünnungen erfährt die Sonde eine anders geartete Umgebung mit stark steigender Polarität. Bei höherer Verdünnung können sich die Acetonitrilmoleküle vermehrt in die mikroskopische Umgebung der Sonde begeben und lösen das Ionennetzwerk auf. Durch die dielektrische Spektroskopie hat BUCHNER ein vergleichbares Phänomen bei der Betrachtung von binären Mischungen von $\left[\mathrm{C}_{2} \mathrm{mim}\right]^{+}\left[\mathrm{EtSO}_{4}\right]^{-}$und Dichlormethan gefunden. Hier bleiben die dielektrischen Eigenschaften bis zu einer Verdünnung von $\mathrm{x}_{I L}=0.2$ erhalten. 118

Die gleiche ionische Flüssigkeit, die BUCHNER in Mischungen mit Dichlormethan untersucht hat, zeigt in der transienten Absorptionsspektroskopie in Mischungen mit Acetonitril ein etwas anderes Bild. Wie in Abbildung 5.31 zu erkennen ergibt sich ein eher kontinuierlicher Verlauf der mikroskopischen Polarität unter Zugabe von Acetonitril. Hier scheint das Ionennetzwerk schon relativ früh aufgebrochen zu werden und die mikroskopische Wechselwirkung mit der Sonde ist offenbar als richtige Mischung aus beiden Komponenten zu betrachten.

Einige Mischungen von $\left[\mathrm{C}_{6} \mathrm{mim}\right]^{+}\left[\mathrm{Tf}_{2} \mathrm{~N}\right]^{-}$und Acetonitril sind auch mit der PSCP-Technik untersucht worden. Die Ergebnisse lassen sich nicht einfach mit dem Befund des Zweifarben-Pump-Probe Experiments vergleichen. Bei dieser Technik wird die Relaxation des $\mathrm{S}_{1} /$ ICT-Zustands global über einen weiten Wellenlängenbereich betrachtet. Hierbei hat sich ein biexponentieller Verlauf dieser Relaxation ergeben, doch die Abhängigkeit von 
der Art des Lösungsmittels wird auch hier deutlich. Eine zusätzliche Dynamik, die einer Solvensrelaxation zugeordnet werden kann, ergibt sich aus einer Blauverschiebung der ESA-Banden des Carotinoids in den binären Mischungen mit der Zeit. Für die spektrale Entwicklung und die kinetischen Spuren können jeweils zwei Zeitkonsten extrahiert werden, die in Tabelle 5.4 zusammengefasst sind und im folgenden dikutiert werden sollen.

In Abbildung 5.32 ist die Abhängigkeit der beiden Zeitkonstanten aus einer globalen biexponentiellen Anpassung der PSCP-Messungen von der Zusammensetzung der binären Mischungen von $\left[\mathrm{C}_{6} \mathrm{mim}\right]^{+}\left[\mathrm{Tf}_{2} \mathrm{~N}\right]^{-}$und Acetonitril gezeigt. Bei beiden Zeitkonstanten ergibt sich ein linearer Verlauf mit zunehmender Konzentration der ionischen Flüssigkeit. Dies steht im Einklag mit den Ergebnissen der Zweifarben-Transienten-Absorptionsspektroskopie (siehe Abbildung 5.28).
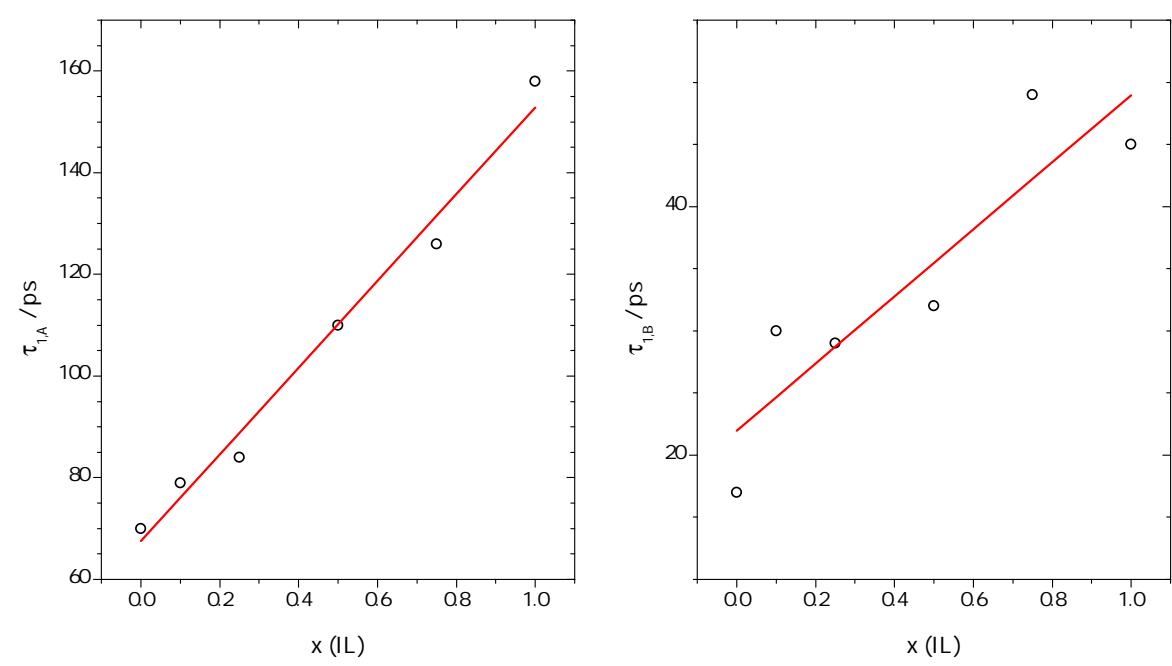

Abbildung 5.32: Abhängigkeit der Lebensdauern aus einer globalen biexponentiellen Anpassung von der Zusammensetzung einer Mischung von $\left[\mathrm{C}_{6} \mathrm{mim}\right]^{+}\left[\mathrm{Tf}_{2} \mathrm{~N}\right]^{-}$und Acetonitril.

Die bestimmten Lebensdauern können ebenfalls mit der effektiven Polaritätsfunktion korreliert werden. Für die langsamere Lebensdauer $\tau_{1, A}$ ergibt sich ein ähnlicher Verlauf, wie in Abbildung 5.28 für die selben Mischungen gezeigt werden konnte. Es kommt ebenfalls bis zu einer hohen Verdünnung von $\mathrm{x}_{I L} \approx 0.2$ nur zu einer geringen Änderung der mikroskopischen Polarität. Die zweite Zeitkonstante $\tau_{1, B}$ zeigt einen anderen Verlauf. Hierbei scheint es sich um einen Relaxationsprozess zu handeln, der zwar von der Polarität des Lösungsmittels abhängig ist, aber nicht so sehr von der mikroskopischen Struktur der Solvensumgebung von 12 'CA. Dies könnten langreichweitende Wechselwirkungen der Kom- 
ponenten von den Mischungen mit der Sonde sein.
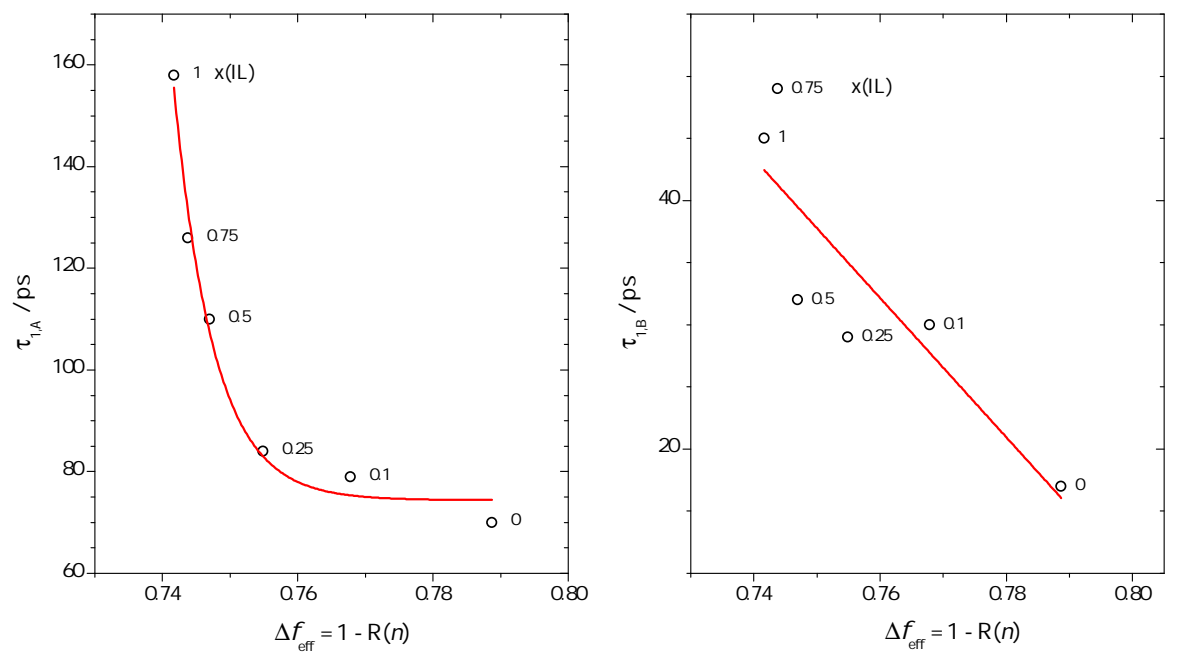

Abbildung 5.33: Polaritätsskala von $12^{\prime} \mathrm{CA}$ in binären Mischungen von $\left[\mathrm{C}_{6} \mathrm{mim}\right]^{+}\left[\mathrm{Tf}_{2} \mathrm{~N}\right]^{-}$und Acetonitril aus PSCP-Daten.

Aus der spektralen Verschiebung der ESA-Banden mit der Zeit können durch eine globale Analyse zwei weitere Zeitkonstanten extrahiert werden. Diese Zeitkonstanten sind in Tabelle 5.4 aufgeführt. Die Bestimmung hat sich aufgrund von Schwankungen während der Messungen als relativ schwierig erwiesen. Es kann keine Korrelation dieser Zeitkonstanten mit der Zusammensetzung der binären Mischungen gefunden werden. Es gibt nur eine klare Tendenz der starken Abnahme mit der Zugabe von Acetonitril. Diese spektrale Dynamik lässt sich wahrscheinlich auf eine Solvensrelaxation zurückführen. In reinem Acetonitril ist diese Relaxation wesentlich schneller als in der ionischen Flüssigkeit.

Die Mischungen von ionischen Flüssigkeiten mit organischen Lösungsmitteln zeigen interessante Eigenschaften. Wie die Messungen der mikroskopischen Umgebung der Sonde 12 'CA zeigen, ist es möglich, die ionischen Flüssigkeiten zu verdünnen ohne ihren Charakter wesentlich zu beeinträchtigen. Dies könnte sehr interessant für die organische Synthese sein. So könnten katalytische Wirkungen der ionischen Flüssigkeiten durch das Mischen mit organischen Lösungsmitteln erhalten bleiben und gleichzeitig deren Viskosität herabgesetzt werden, was einen technischen Vorteil bei der Produktion ergeben könnte. Neben dem Einsatz der ionischen Flüssigkeiten in der Synthesechemie, gibt es weitere interessante Anwendungsmöglichkeiten. So werden sie als mögliche Elektrolyte in Farbstoffsolarzellen getestet. Es konnte gezeigt werden, dass sie sich sehr wohl für dieses Anwendungsgebiet 
eignen, doch sind die wichtigen Prozesse in den Solarzellen noch nicht so gut verstanden. Ein wichtiger Aspekt ist die Wechselwirkung der Farbstoffe mit dem Elektrolyten. Dies ist der Ansatz des nun folgenden Kapitels.

Tabelle 5.4: Brechungsindices und Zeitkonstanten der binären Mischungen

\begin{tabular}{|c|c|c|c|c|c|c|c|c|}
\hline \multirow[t]{2}{*}{$x(\mathrm{IL})$} & \multirow[t]{2}{*}{$n$} & \multicolumn{2}{|c|}{ TA } & \multicolumn{5}{|c|}{ PSCP } \\
\hline & & $\tau_{1}[\mathrm{ps}]$ & $\tau_{3}[\mathrm{ps}]$ & $\tau_{1, A}[\mathrm{ps}]$ & $\tau_{1, B}[\mathrm{ps}]$ & $\beta$ & $\tau_{l}$ [ps] & $\tau_{s}[\mathrm{ps}]$ \\
\hline \multicolumn{9}{|c|}{$\left[\mathrm{C}_{6} \mathrm{mim}^{+}\left[\mathbf{T f}_{2} \mathbf{N}\right]^{-}\right.$} \\
\hline 0 & 1.343 & 42 & 0.4 & 70 & 17 & 0.48 & 4.1 & 1.6 \\
\hline 0.05 & 1.365 & 38 & 0.8 & & & & & \\
\hline 0.1 & 1.381 & 38 & 1.1 & 79 & 30 & 0.23 & 4.4 & 0.2 \\
\hline 0.15 & 1.392 & 43 & 1.7 & & & & & \\
\hline 0.2 & 1.399 & 45 & 1.3 & & & & & \\
\hline 0.25 & 1.406 & & & 84 & 29 & 0.26 & 103 & 0.2 \\
\hline 0.3 & 1.410 & 56 & 1.9 & & & & & \\
\hline 0.4 & 1.416 & 66 & 2.1 & & & & & \\
\hline 0.5 & 1.420 & 73 & 2.0 & 110 & 32 & 0.47 & 17 & 0.1 \\
\hline 0.6 & 1.423 & 83 & 2.8 & & & & & \\
\hline 0.75 & 1.426 & & & 126 & 49 & 0.37 & 65 & 0.1 \\
\hline 0.8 & 1.427 & 85 & 2.8 & & & & & \\
\hline 0.9 & 1.429 & 90 & 2.4 & & & & & \\
\hline 1.0 & 1.430 & 91 & 2.5 & 158 & 45 & 0.33 & 32 & 0.1 \\
\hline \multicolumn{9}{|c|}{$\left[\mathrm{C}_{2} \mathrm{mim}\right]^{+}\left[\mathrm{EtSO}_{4}\right]^{-}$} \\
\hline 0 & 1.343 & 42 & 0.4 & & & & & \\
\hline 0.05 & 1.364 & 50 & 0.7 & & & & & \\
\hline 0.1 & 1.386 & 46 & 0.9 & & & & & \\
\hline 0.2 & 1.418 & 62 & 1.5 & & & & & \\
\hline 0.5 & 1.453 & 65 & 1.8 & & & & & \\
\hline 0.8 & 1.471 & 87 & 2.0 & & & & & \\
\hline 0.9 & 1.475 & 93 & 1.0 & & & & & \\
\hline 1.0 & 1.478 & 87 & 1.0 & & & & & \\
\hline
\end{tabular}




\section{Kapitel 6}

\section{Indolinbasierte Farbstoffe für Solarzellen}

Farbstoffsolarzellen (Dye-Sensitized Solar Cells (DSSCs)) sind beliebt in der Grundlagenforschung sowie für praktische Anwendungen. Das Interesse ist sehr hoch, da sie gegenüber den Siliziumsolarzellen einige Vorteile bieten. Die Herstellungskosten sind relativ niedrig, die Produktion an sich ist sehr einfach, und es können flexible und transparente Module hergestellt werden. Die Stabiltät und der Wirkungsgrad dieser Solarzellen ist in den vergangenen Jahren stetig verbessert worden. $\underline{34[125}$

In diesem Kapitel werden erste Charakterisierungen von indolinbasierten Farbstoffen in organischen Lösungsmitteln sowie in ionischen Flüssigkeiten mittels ultraschneller transienter Absorptionsmethoden präsentiert. Diese Klasse von Farbstoffen wird aktuell als möglicher metallfreier Sensibilisator für DSSCs getestet. ${ }^{[125126}$ Aufgrund der positiven Eigenschaften von ionischen Flüssigkeiten, wie z. B. den quasivernachlässigbaren Dampfdruck, werden diese als mögliche Elektrolyten diskutiert. In den DSSCs treten viele Komponenten auf, die aufeinander abgestimmt werden müssen, um einen hohen Wirkungsgrad erreichen zu können. Wie die Praxis zeigt, funktioniert dieser Typ von Solarzelle hervorragend, die genauen molekularen Abläufe sind allerdings noch nicht hinreichend bekannt. Besonders die Wechselwirkung zwischen dem Sensibilisator und dem Elektrolyten kann entscheidend sein für die optimale Umsetzung in einer Farbstoffsolarzelle. Diesem wichtigen Aspekt widmen sich die nun folgenden Kapitel. 


\subsection{Prinzip der DSSC}

Die Idee der DSSCs stammt von GRÄTZEL, daher werden sie auch "Grätzel-Zellen“ genannt. Im Gegensatz zu den Siliziumsolarzellen, in denen die Lichtabsorption durch den Halbleiter erfolgt, haben die DSSCs ein abweichendes Funktionsprinzip. Es kommen Farbstoffpigmente zum Einsatz, die für die Lichtabsorption verantwortlich sind. Diese Sensibilisatoren sind auf einer porösen Halbleiterschicht aufgebracht. Hierbei werden Oxide, wie z. B. Titanoxid $\left(\mathrm{TiO}_{2}\right)$ aber auch Zink- und Nioboxid verwendet. Wie in Abbildung $6.1 \mathrm{zu}$ sehen, hat der Farbstoff, welcher sich in monomolekularer Schicht auf dem Halbleiter befindet, die Aufgabe Licht zu absorbieren. Dabei geht er in einen elektronisch angeregten Zustand über und kann ein Elektron in das Leitungsband des Halbleiters transferieren. Das Elektron wird dann durch den Halbleiter an eine Kontaktelektrode geleitet. Diese Elektrode muss aus einem lichtdurchlässigen Material bestehen und zugleich leitend sein. Daher besteht sie meist aus Glas, welches mit einer dünnen Lage von beispielsweise Indium-Zinn-Oxid beschichtet ist. Als Gegenelektroden werden meist platinbeschichtete Materialien verwendet. So kann die aus dem Licht gewonnene Energie durch den Elektronenfluss an einen Verbraucher weiter gegeben werden. Damit dieser Prozess ständig wiederholt werden kann, muss der Sensibilisator wieder reduziert werden. Der Ladungstransport erfolgt über einen Elektrolyten, der aus einem organischen Lösungsmittel bzw. einer ionischen Flüssigkeit mit enthaltenem Redox-Paar (Iodid/Triiodid) besteht. Hierbei wird das Triiodid an der Platinelektrode zu Iodid reduziert, welches wiederum seine Ladung an den Farbstoff abgeben kann. 127:128

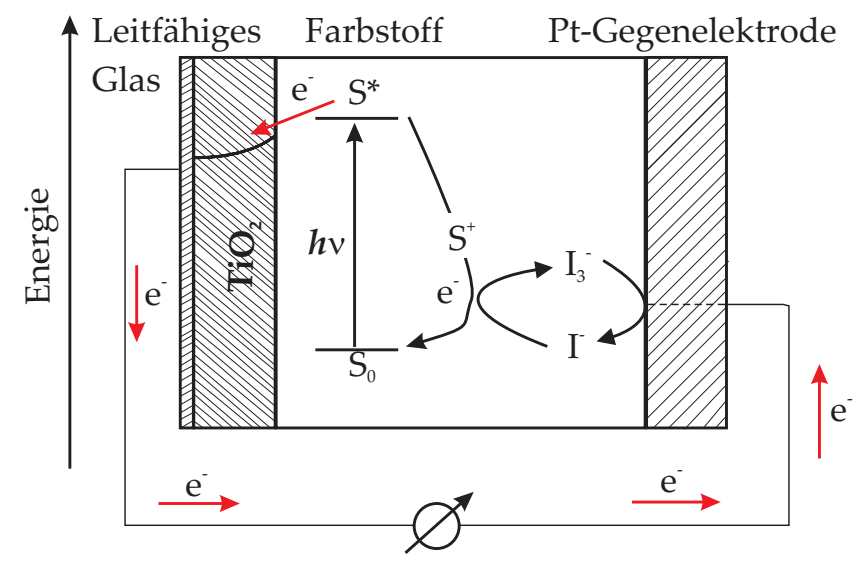

Abbildung 6.1: Prinzip der Dye-Sensitized Solar Cells (DSSCs). 


\subsection{Sensibilatoren und Elektrolyte}

Der Wirkungsgrad der DSSCs liegt in der Größenordnung von $10 \%{ }^{[125]}$ Entscheidend hierbei ist natürlich das eingesetzte Material unter anderem auch die Wahl des Sensibilators und des Elektrolyten. Der angegebene Wirkungsgrad bezieht sich auf DSSCs mit organischen Lösungsmitteln wie z. B. Acetonitril als Elektrolyten. Legt man Wert auf eine Langzeitstabilität der Solarzellen, dann sollte ein Ersatz für die organischen Lösungsmittel gefunden werden. Ionische Flüssigkeiten mit ihrer hohen thermischen Stabilität und guten Leitfähigkeit, die im Gegensatz zu den organischen Flüssigkeiten nicht verdampfen, stellen hierbei eine interessante Alternative dar. Als Redoxpaar hat sich das System Iodid/Triiodid als besonders effizient erwiesen. Es gibt bereits einige Ergebnisse von DSSCs auf Basis von ionischen Flüssigkeiten mit Ruthenium-Komplexen als Sensibilisatoren. ${ }^{1291130}$ Die Verwendung dieser Farbstoffe bringt bisher die höchste Effizienz für die DSSCs. Als Beispiel eines solchen Farbstoffs ist der Ruthenium-Komplex N719 in Abbildung 6.2 gezeigt.

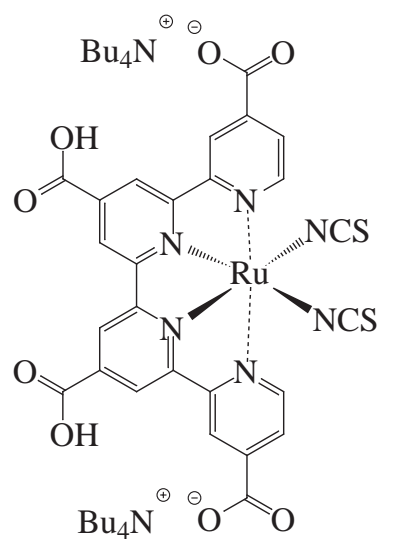

Abbildung 6.2: Chemische Struktur von N719.

Die Umwandlung von Licht in elektrische Ladung ist bei der Benutzung von ionischen Flüssigkeiten noch nicht so effizient wie mit organischen Lösungsmitteln. Der Grund dafür ist die hohe Viskosität der ILs, was zu schlechteren Transporteigenschaften führt. Die positiven Eigenschaften der ionischen Flüssigkeiten möchte man allerdings nicht missen, daher ist eine Weiterentwicklung der Sensibilatoren gefordert. Die Anforderungen für einen Farbstoff sind sehr hoch. Es ist eine möglichst vollständige Absorption des sichtbaren bis nahinfraroten Bereich des elektromagnetischen Spektrums gewünscht. Außerdem müssen die Farbstoffe möglichst gut an der Oberfläche vom Halbleiter binden, was durch geeignete 
funktionelle Gruppen gewährleistet wird. Metallfreie organische Sensibilatoren scheinen diesen Anforderungen gewachsen und könnten dazu beitragen, den Wirkungsgrad von IL-basierten Farbstoffsolarzellen zu steigern. Sie haben im sichtbaren Bereich einen höheren Extinktionskoeffizienten als die Rutheniumfarbstoffe, dass heißt sie können das Licht besser sammeln. Ein Beispiel sind die indolinbasierten Farbstoffe, die in dieser Arbeit untersucht worden sind.

Die molekulare Struktur der Farbstoffe D102, D131, D149 und D205 kann Abbildung 6.3 entnommen werden. Die Farbstoffe D149 und D205 unterscheiden sich von D102 durch eine zusätzliche Rhodanineinheit. Ersetzt man den Ethylrest des Rhodanins von D149 durch einen Oktylrest erhält man D205. Der Farbstoff D131 unterscheidet sich von den anderen durch die Abwesenheit einer Rhodanineinheit. Die Endgruppe, die hier für das Andocken an den Halbleiter Verantwortung trägt, ist ein Nitril- und ein Carbonsäurerest. Die erhöhte $\pi$-Konjugation führt zu einer Rotverschiebung D131 $\rightarrow$ D102 $\rightarrow$ D149 $\approx$ D205 im sichtbaren Spektrum.

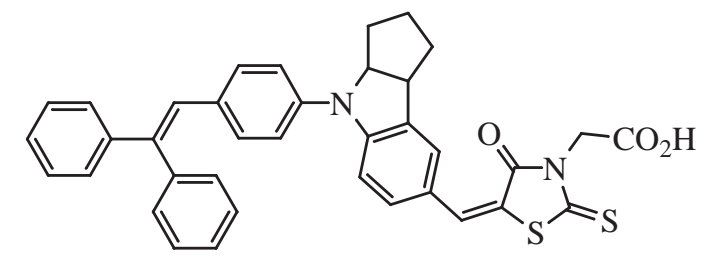

D102

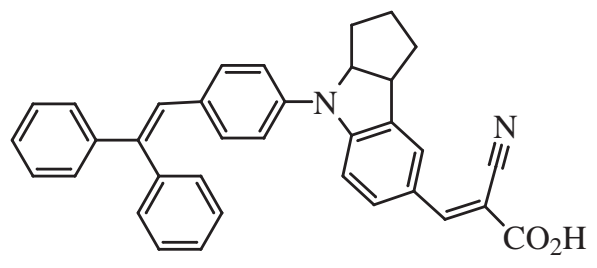

D131<smiles>CCN1C(=O)/C(=c2/s/c(=C/c3ccc4c(c3)C3CCCC3N4c3ccc(C=C(c4ccccc4)c4ccccc4)cc3)c(=O)n2CC(=O)O)SC1=S</smiles>

D149<smiles>CCON1C(=O)/C(=c2/s/c(=C/c3ccc4c(c3)C3CCCC3N4c3ccc(C=C(c4ccccc4)c4ccccc4)cc3)c(=O)n2CC(=O)O)SC1=S</smiles>

D205

Abbildung 6.3: Chemische Struktur der indolinbasierten Farbstoffe.

Die Benutzung von indolinbasierten Farbstoffen mit ionischen Flüssigkeiten als Elektrolyt führte zu vielversprechenden DSSCs. Im besonderen werden ionische Flüssigkeiten 
mit Tetracyanoboratanion benutzt. ${ }^{125}$ Über die genaue mikroskopische Wechselwirkung der Farbstoffe mit den Elektrolyten ist bisher noch nichts bekannt. Durch die ultraschnelle laserspektroskopische Untersuchung dieser Farbstoffe zunächst in organischen Lösungsmitteln aber auch in ionischen Flüssigkeiten, unter anderem in einer tetracyanoboratbasierten IL, können erste Einblicke in diese mikroskopischen Wechselwirkungen gewonnen werden.

\subsection{Ultraschnelle intramolekulare Prozesse von indolinbasierten} Farbstoffen

Der erste Schritt im Betrieb von DSSCs ist die Absorption von Photonen. Diese regen ein Elektron des Farbstoffs in ein höher gelegenes elektronisches Niveau an. Das Elektron kann dann in das Leitungsband des Halbleiters transferiert werden. Die indolinbasierten Farbstoffe dieser Arbeit bestehen aus einer Elektronendonor- und einer ElektronenakzeptorEinheit. Die beiden Einheiten sind über ein konjugiertes $\pi$-System kovalent miteinander verknüpft. Alle hier betrachteten Farbstoffe haben die gleiche Donorgruppe, ein substituiertes Indolin. Die Akzeptoren unterscheiden sich, wie in Abbildung 6.3 ersichtlich wird.

Im Gegensatz zu den Apocarotinoiden ist der Übergang aus dem elektronischen Grundzustand $\left(S_{0}\right)$ in den ersten angeregten Zustand $\left(S_{1}\right)$ nicht symmetrieverboten. Neben der Anregung in diesen Zustand kann auch der Übergang in den zweiten angeregten Zustand $\left(\mathrm{S}_{2}\right)$ erfolgen. Zeitabhängige DFT-Rechnungen der Indolinfarbstoffe konnten zeigen, dass der $\mathrm{S}_{1}$-Zustand einen intramolekularen Charge-Transfer-Charakter (ICT) hat. Das Dipolmoment dieses angeregten Zustands ist ca. $30 \mathrm{D}$ höher als vom Grundzustand. ${ }^{131}$ Daher kommt man bei der Photophysik der indolinbasierten Farbstoffe auf ein ähnliches Bild, wie bei den Apocarotinoiden. In Abbildung 6.4 ist eine schematische Darstellung der elektronischen Zustände zu sehen.

Nach der Anregung in den $\mathrm{S}_{2}$-Zustand erfolgt eine innere Konversion (IC) in den ersten angeregten Zustand $\mathrm{S}_{1}$. Diese IC ist langsamer als bei den Apocarotinoiden und wird durch die Zeitkonstante $\tau_{2}$ beschrieben. Nach der Population des $S_{1}$-Zustands erfolgt eine weitere IC mit der Zeitkonstanten $\tau_{1}$ zurück in den Grundzustand. Aufgrund des ICT-Charakters von $S_{1}$ ergeben sich solvatochrome Effekte. Die relative Lage von $S_{1}$ und $S_{0}$ wird durch das 


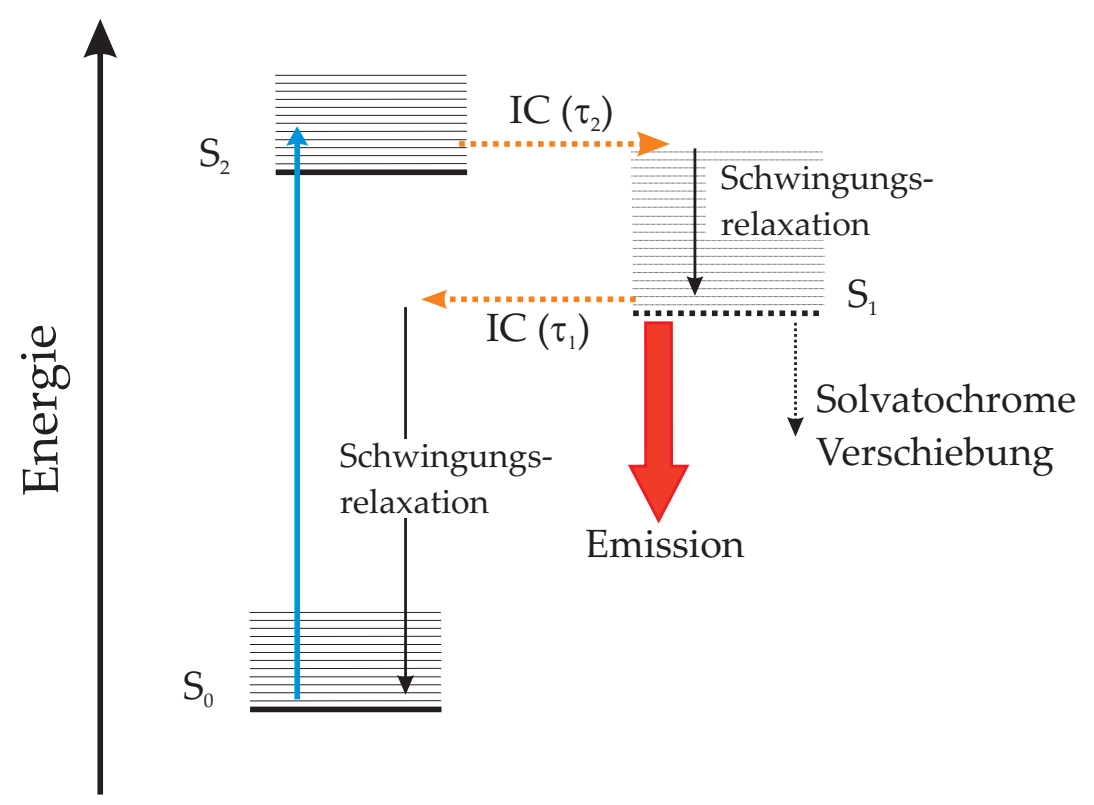

Abbildung 6.4: Drei-Zustände-Modell der indolinbasierten Farbstoffe.

Lösungsmittel beeinflusst. Dies ist in Analogie zu den Apocarotinoiden.

Die Zeitkonstanten der inneren Konversionen $S_{2} \rightarrow S_{1}\left(\tau_{2}\right)$ und $S_{1} \rightarrow S_{0}\left(\tau_{1}\right)$ können mittels der zeitaufgelösten Pump-Probe-Methoden ermittelt werden. Hierbei wird das gleiche kinetische Modell wie bei den Apocarotinoiden angenommen. Zusätzlich zu den beiden Zeitkonstanten ist hier ein dritter Term enthalten, der die Solvensrelaxation beschreibt. Beim Aufbau der $S_{1}$-Population erkennt man in den Signalen eine zusätzliche Dynamik, die ebenfalls vom Lösungsmittel abhängig ist. Den transienten Spektren ist auch hier eine Excited-State Absorption (ESA) aus beiden angeregten Zuständen und besonders im Fall der TA-Messungen eine stimulierte Emission aus dem $S_{1}$-Zustand zu entnehmen.

Das Verständnis der ultraschnellen Dynamik dieser Farbstoffe, welche eine entscheidende Komponente in DSSCs darstellen, kann zur Verbesserung der Solarzellen beitragen. Der Fokus liegt hierbei auf der Lebensdauer $\tau_{1}$ und deren Abhängigkeit vom Lösungsmittel. In den Solarzellen werden die Farbstoffe durch das Sonnenlicht hauptsächlich in den $S_{1}$ Zustand angeregt. Die IC in den Grundzustand steht dann in Konkurrenz zur Elektronenübertragung in den Halbleiter. Aufgrund der Solvensabhängigkeit könnte es möglich sein diese Prozesse gezielt zu steuern. Durch die Wahl des Lösungsmittels könnte die Quantenausbeute für die Elektroneninjektion gesteigert werden, indem beispielsweise $\tau_{1}$ erhöht wird. 


\subsection{Experimentelle Ergebnisse für D102, D131, D149 und D205}

In den nachfolgenden Kapiteln werden die Ergebnisse der stationären Absorptions- sowie der transienten Absorptionsspektroskopie präsentiert. Homogene Lösungen sind in einem Ultraschallbad hergestellt worden. Die Konzentration der Farbstoffe betrug $2 \cdot 10^{-4} \mathrm{~mol}$. $\mathrm{L}^{-1}$. In Methanol und Acetonitril sind die Konzetrationen geringer, da die Löslichkeit hier niedriger ist und die Farbstoffe filtriert werden mussten. Die Löslichkeit in ionischen Flüssigkeiten ist besser als die von Apocarotinoiden.

In Abschnitt 6.4.1 werden die stationären Absorptionsspektren von D102, D131, D149 und D205 in verschiedenen Lösungsmitteln gezeigt. Dem schließen sich in Abschnitt 6.4.2 und 6.4.3 die Messungen der ultraschnellen Dynamik an. Die Ergebnisse werden schließlich in Abschnitt 6.5 diskutiert.

\subsubsection{Stationäre Absorptionsspektroskopie}

In Abbildung 6.5 sind die stationären Absorptionsspektren der indolinbasierten Farbstoffe in verschiedenen organischen Lösungsmitteln gezeigt. Es ist jeweils ein Ausschnitt der $S_{1}$-Bande zu sehen. Für ein gesamtes Spektrum der Farbstoffe in Methanol sei auf Abbildung 3.12 verwiesen. Aus den Spektren wird ersichtlich, dass es zu einer solvatochromen Verschiebung kommt. Auch ist die Lage des Absorptionsmaximums von der chemischen Identität des Farbstoffs abhängig. D149 und D205 unterscheiden sich lediglich in der Kettenlänge des Seitenrestes und haben beide ein Absorptionsmaximum von etwa $530 \mathrm{~nm}$. D102 besitzt einen Rhodaninrest weniger und ist $30 \mathrm{~nm}$ ins Blaue verschoben, das Absorptionsmaximum liegt bei $500 \mathrm{~nm}$. Strukturell unterscheidet sich D131 deutlich von den anderen Farbstoffen durch die Abwesenheit von Rhodaninresten. Dieser Farbstoff ist am weitesten blauverschoben und hat ein Absorptionsmaximum von ca. $450 \mathrm{~nm}$. Eine genaue Gegenüberstellung der Absorptionsmaxima in Abhängigkeit vom Lösungsmittel ist zusammen mit den Ergebnissen der transienten Absorptionsmessungen in Tabelle 6.1 aufgeführt. Abschnitt 6.4 .3 behandelt die Dynamik von D149 in ionischen Flüssigkeiten. Die stationären Spektren der gemessenen ILs $\left[\mathrm{C}_{4} \mathrm{mim}\right]^{+}\left[\mathrm{B}(\mathrm{CN})_{4}\right]^{-}$und $\left[\mathrm{C}_{2} \mathrm{mim}\right]^{+}\left[\mathrm{N}(\mathrm{CN})_{2}\right]^{-}$zeigen eine relativ starke Rotverschiebung von $\left[\mathrm{C}_{2} \mathrm{mim}\right]^{+}\left[\mathrm{N}(\mathrm{CN})_{2}\right]^{-}$im Vergleich $\mathrm{zu}\left[\mathrm{C}_{4} \mathrm{mim}\right]^{+}$ $\left[\mathrm{B}(\mathrm{CN})_{4}\right]^{-}$, welche ein ähnliches Absorptionsmaximum wie Ethanol besitzt. 

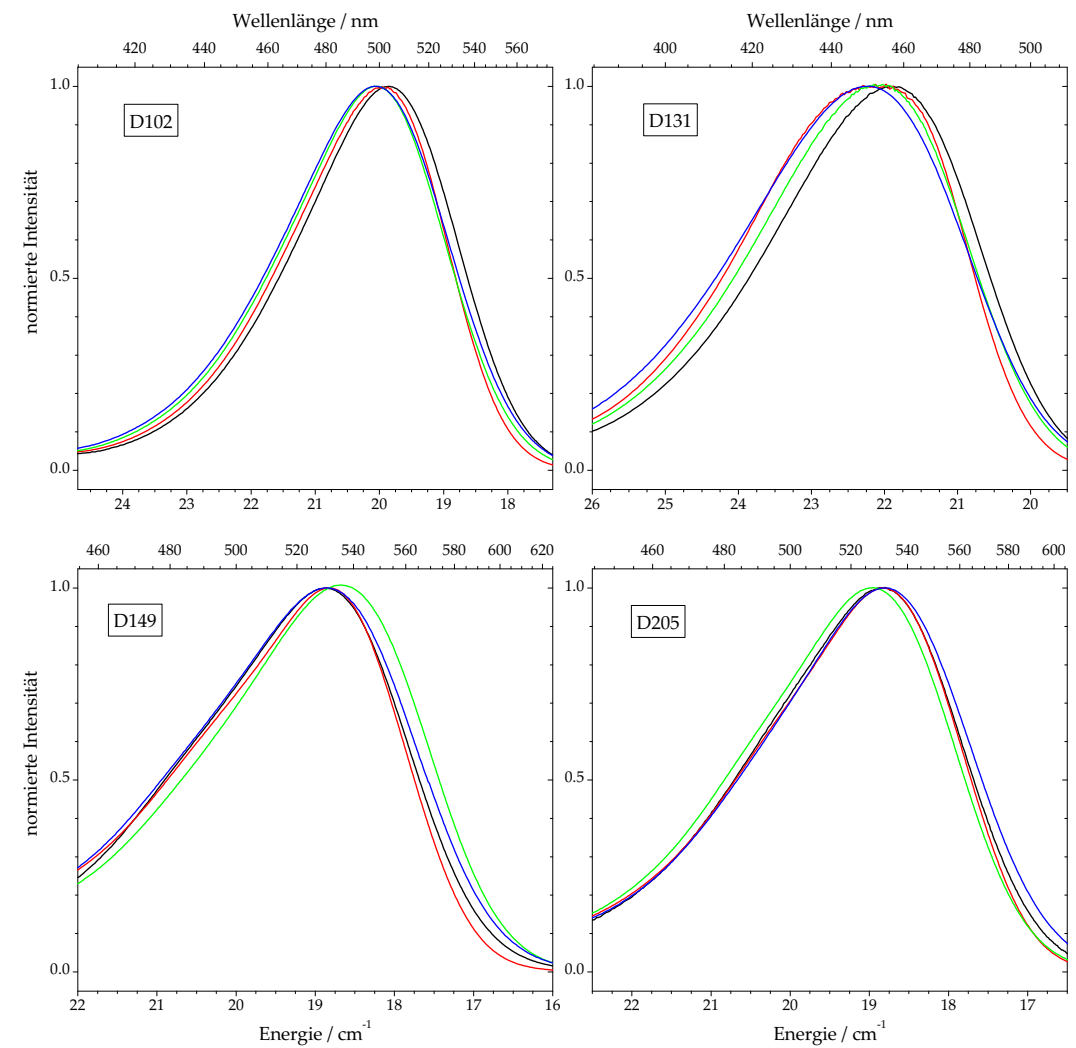

Abbildung 6.5: Stationäre Absorptionsspektren von D102, D131, D149 und D205. Die Messungen wurden in unterschiedlichen organischen Lösungsmitteln durchgeführt: - Acetonitril, - Tetrahydrofuran, - Ethanol und - Methanol.

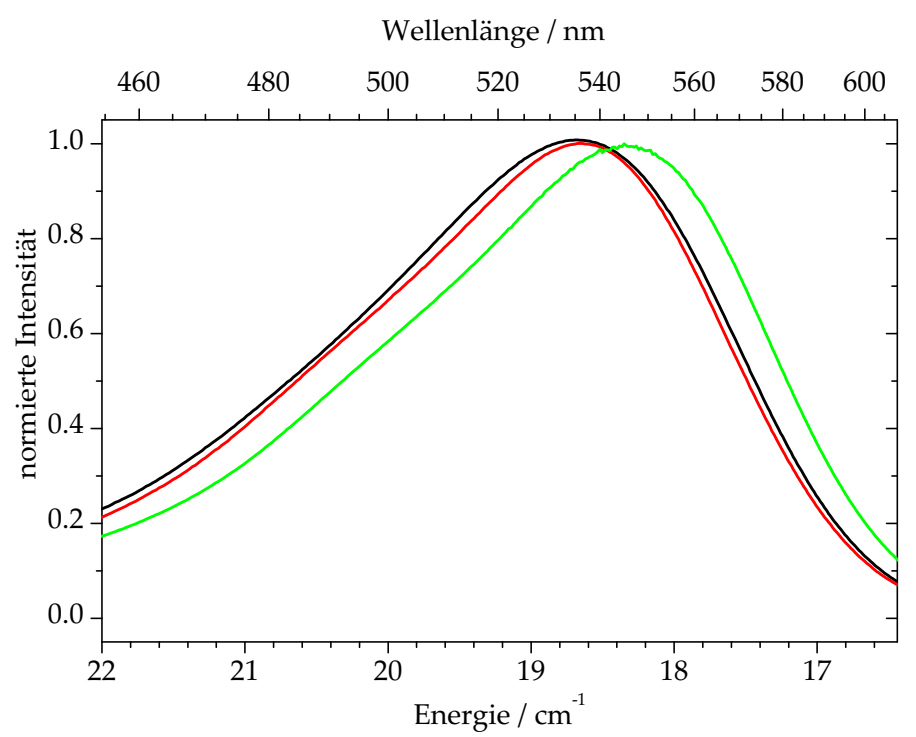

Abbildung 6.6: Stationäre Absorptionsspektren von D149 in - Ethanol, $-\left[\mathrm{C}_{4} \mathrm{mim}\right]^{+}\left[\mathrm{B}(\mathrm{CN})_{4}\right]^{-}$ und $-\left[\mathrm{C}_{2} \mathrm{mim}\right]^{+}\left[\mathrm{N}(\mathrm{CN})_{2}\right]^{-}$. 


\subsubsection{Transiente Absorptions- (TA) Spektroskopie}

Die transienten Absorptionssignale im nahen Infrarotbereich von D102, D131, D149 und D205 sind in den Abbildungen 6.7 bis 6.10 gezeigt. Es sind jeweils Messungen in Acetonitril, Tetrahydrofuran, Ethanol und Methanol durchgeführt worden. Die Farbstoffe sind mit $375 \mathrm{~nm}$ angeregt und mit $750 \mathrm{~nm}$ abgefragt worden. Der steile Anstieg am Anfang der Signale resultiert aus der Excited-State Absorption (ESA) des $\mathrm{S}_{2}$-Zustands. Durch innere Konversion (IC) wird der $\mathrm{S}_{1}$-Zustand mit intramolekularem Charge-Transfer Charakter (ICT) bevölkert. Diese IC ist im Vergleich mit den gemessenen Carotinoiden langsam, doch sehr viel schneller als die Relaxation des $S_{1}$-Zustands, was wiederum in Relation mit den Carotinoiden steht. Der Aufbau der $S_{1}$-Population ist dem Signal durch den Abfall bis hin zu negtiven Werten zu entnehmen. Zusätzlich ist diesem Abfall eine Solvensdynamik aufgeprägt, welche sich als Krümmung vor dem Abklingen der stimulierten Emission bemerkbar macht. Die stimulierte Emission erfolgt aus dem $S_{1}$-Zustand und deren Abfall kennzeichnet die Relaxation dieses Zustands, wobei der Grundzustand wieder bevölkert wird.

Die transienten Signale werden durch das kinetische Modell $S_{2} \rightarrow S_{1} \rightarrow S_{0}$ beschrieben und mit Gleichung 5.8 (siehe Kapitel 5.1) simuliert. In diesem Modell enthalten sind die Zeitkonstanten $\tau_{2}$ für die Relaxation des $S_{2}$-Zustands, $\tau_{1}$ für den Übergang $S_{1} \rightarrow S_{0}$ und $\tau_{3}$, welche die Dynamik des Lösungsmittels widerspiegelt. Eine Zusammenfassung der kinetischen Anpassung ist Tabelle $6.1 \mathrm{zu}$ entnehmen.

Betrachtet man die Form der transienten Signale, so stellt man fest, dass die Form der THF-Signale eine andere ist. Die transienten Spektren aller gemessenen indolinbasierten Farbstoffe in THF weichen von den anderen organischen Lösungsmitteln ab. Diese Abweichung wird in Abschnitt 6.5 diskutiert. Hier wird ebenfalls ein genauer Vergleich der Farbstoffe erfolgen. 

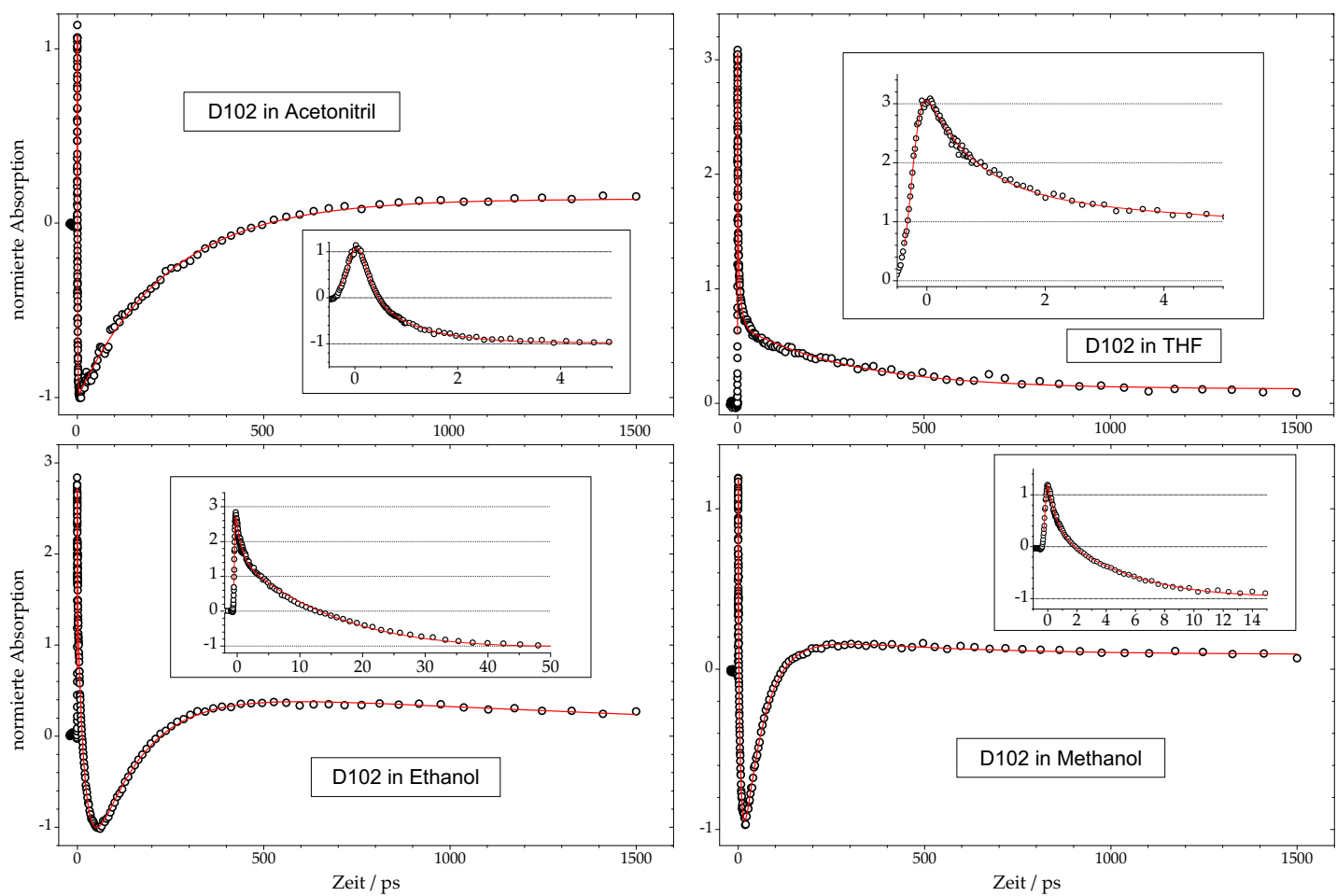

Abbildung 6.7: Transientes Absorptions- / stimuliertes Emissionssignal von D102 in organischen Lösungsmitteln.
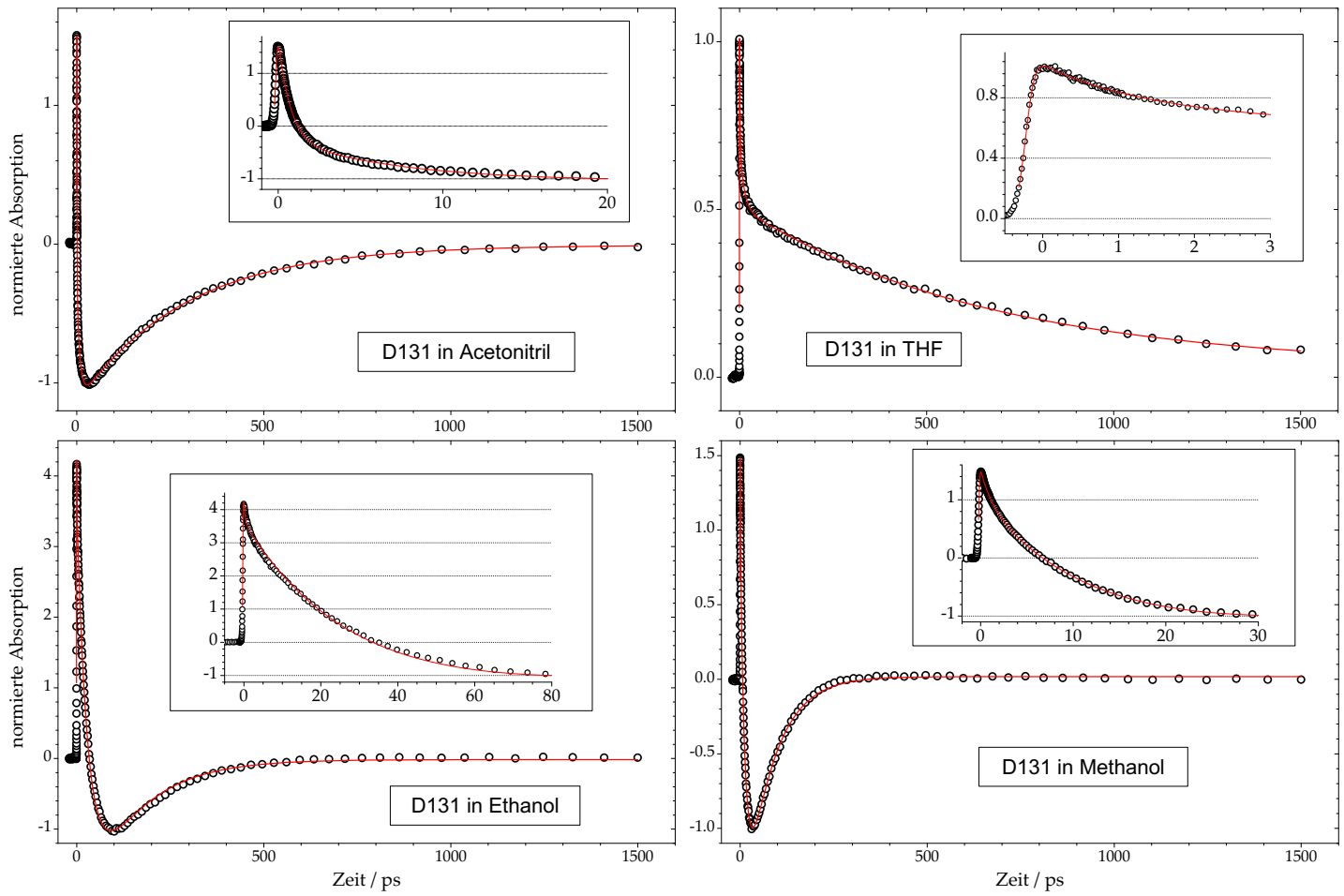

Abbildung 6.8: Wie Abbildung 6.7, aber für D131. 

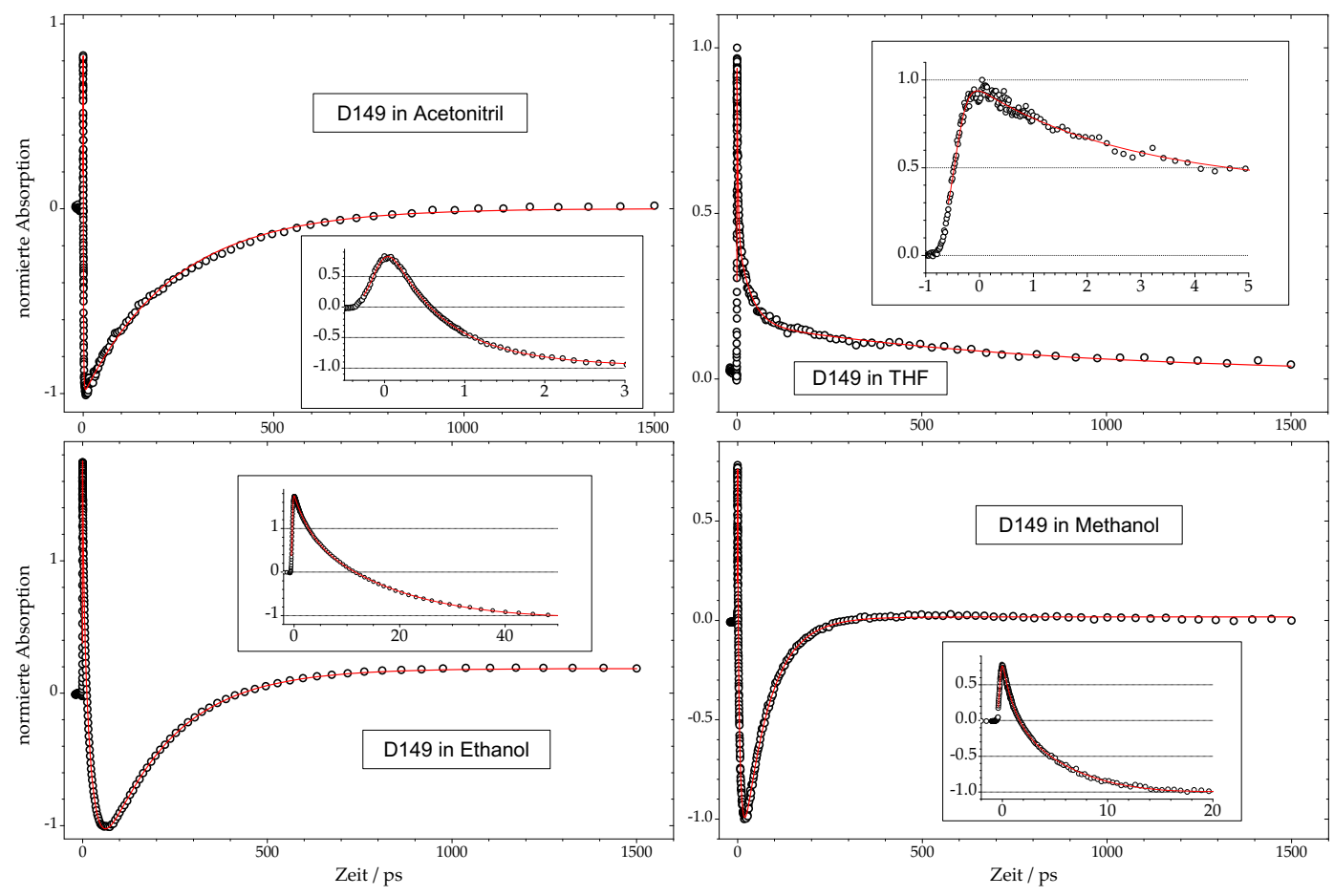

Abbildung 6.9: Wie Abbildung 6.7 aber für D149.
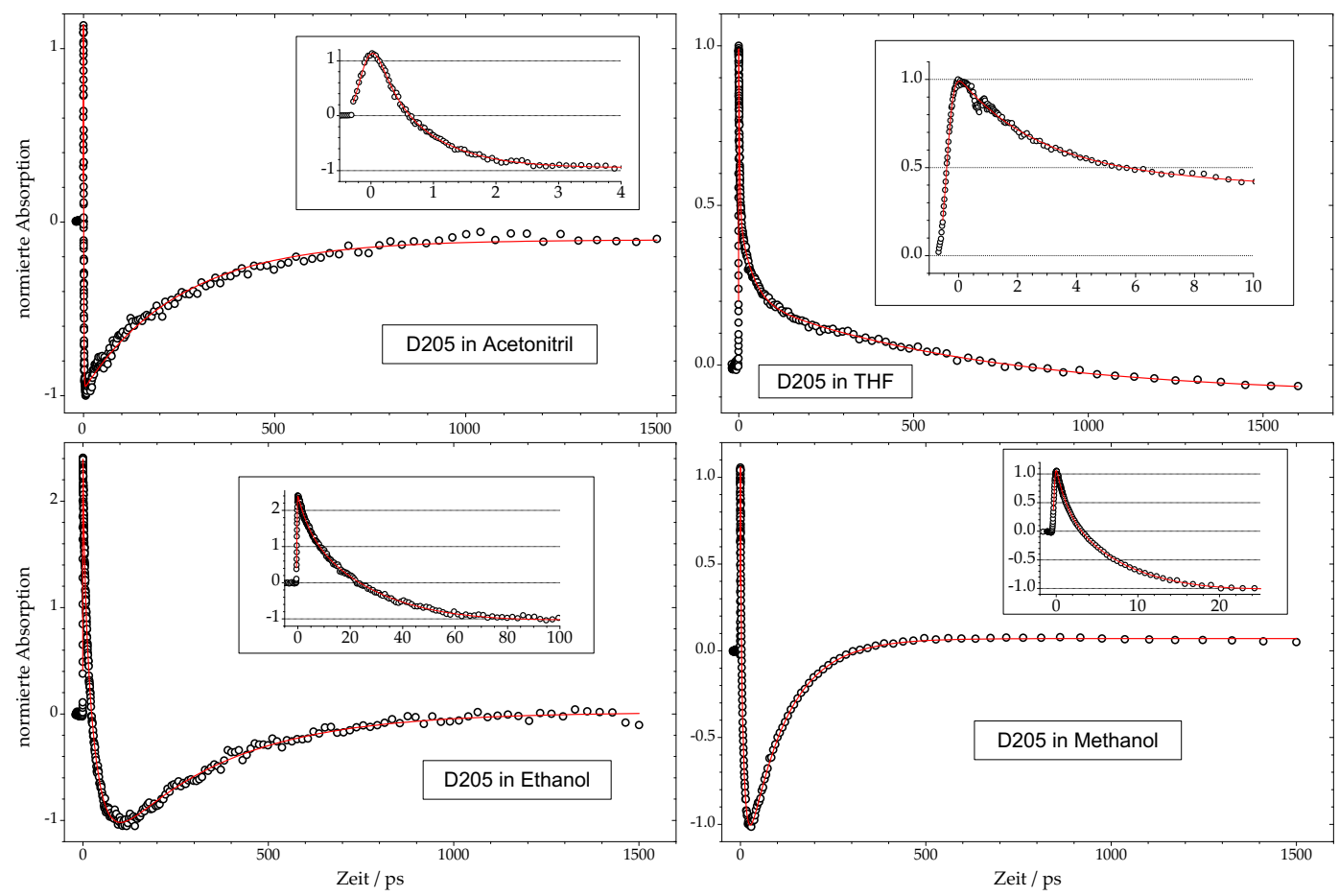

Abbildung 6.10: Wie Abbildung 6.7 aber für D205. 


\subsubsection{Pump-Superkontinuum-Probe-Spektroskopie}

In den Abbildungen 6.11 und 6.12 sind die transienten Spektren von D149 in $\left[\mathrm{C}_{4} \mathrm{mim}\right]^{+}$ $\left[\mathrm{B}(\mathrm{CN})_{4}\right]^{-}$und $\left[\mathrm{C}_{2} \mathrm{mim}\right]^{+}\left[\mathrm{N}(\mathrm{CN})_{2}\right]^{-}$dargestellt. Dieses wird in den PSCP-Messungen mit $480 \mathrm{~nm}$ in den $\mathrm{S}_{1}$-Zustand angeregt. Im oberen Teil der Abbildungen ist die Entwicklung bei frühen Zeiten zu erkennen. Aufgrund der schlechteren Löslichkeit von D149 in $\left[\mathrm{C}_{4} \mathrm{mim}\right]^{+}\left[\mathrm{B}(\mathrm{CN})_{4}\right]^{-}$sind die Signale wesentlich kleiner und sehen stark verrauscht aus. Die Bildung des GSB und der ESA-Banden ist dennoch deutlich zu erkennen. Die transienten Spektren sind hier in Abständen von 40 fs gezeigt. Im mittleren Teil der Abbildung ist die Entwicklung der Banden bei Zeiten unter einer Pikosekunde gezeigt, wohingegen der untere Teil die Relaxation aller Banden bis hin zu 1.5 ns zeigt.

Die PSCP-Spektren von D149 unterscheiden sich deutlich von den Apocarotinoiden. Es sind fünf anstelle von vier Banden erkennbar und die Form ist ebenfalls eine andere. Da die Anregung direkt in den $S_{1}$-Zustand erfolgt, werden im Gegensatz zu den Apocarotinoiden bei frühen Zeiten keine spektralen Beiträge des $\mathrm{S}_{2}$-Zustands beobachtet. In dem etwas deutlicheren Signal von D149 in $\left[\mathrm{C}_{2} \mathrm{mim}\right]^{+}\left[\mathrm{N}(\mathrm{CN})_{2}\right]^{-}$treten bei frühen Zeiten zwei negative Banden auf. Diese sind dem GSB zuzuordenen. Die erste kleinere Bande liegt zwischen 360 und $410 \mathrm{~nm}$ und die weitere größere Bande zwischen 460 und $580 \mathrm{~nm}$. Der Grundzustand ist nach Anregung weniger populiert, daher werden die Probepulse in diesem spektralen Bereich weniger stark absorbiert als vor der Anregung und es resultiert ein negatives Signal. Die GSB-Banden erreichen in Femtosekunden ihr Maximum und werden langsam bis hin zu 1500 ps wieder aufgefüllt. Im unteren Feld sind die jeweiligen stationären Absorptionsspektren gezeigt. Ein Vergleich zeigt, dass diese Spektren dem GSB entsprechen. Sogar das Verhältnis beider Banden ist vergleichbar.

Bei den frühen Zeiten sind erst zwei positive Banden deutlich. Die eine Bande liegt im Bereich von 410 bis $460 \mathrm{~nm}$ und die andere erstreckt sich von 580 bis zum Rand des Spektralbereichs bei $770 \mathrm{~nm}$. Hierbei handelt es sich um Übergänge aus dem $\mathrm{S}_{1}$-Zustand in höher gelegene Niveaus. Im unteren Teil wird dann deutlich, dass sich die rechte ESABande in zwei Banden aufteilt. Im Bereich von 670 bis $770 \mathrm{~nm}$ ist ein langsamer Aufbau von stimulierter Emission zu erkennen, sodass ein weiteres negatives Signal entsteht. Diese stimulierte Emission zusammen mit dem GSB und der ESA-Banden klingen mit der Zeit $\mathrm{ab}$, der $\mathrm{S}_{1}$-Zustand relaxiert zurück in den Grundzustand. 


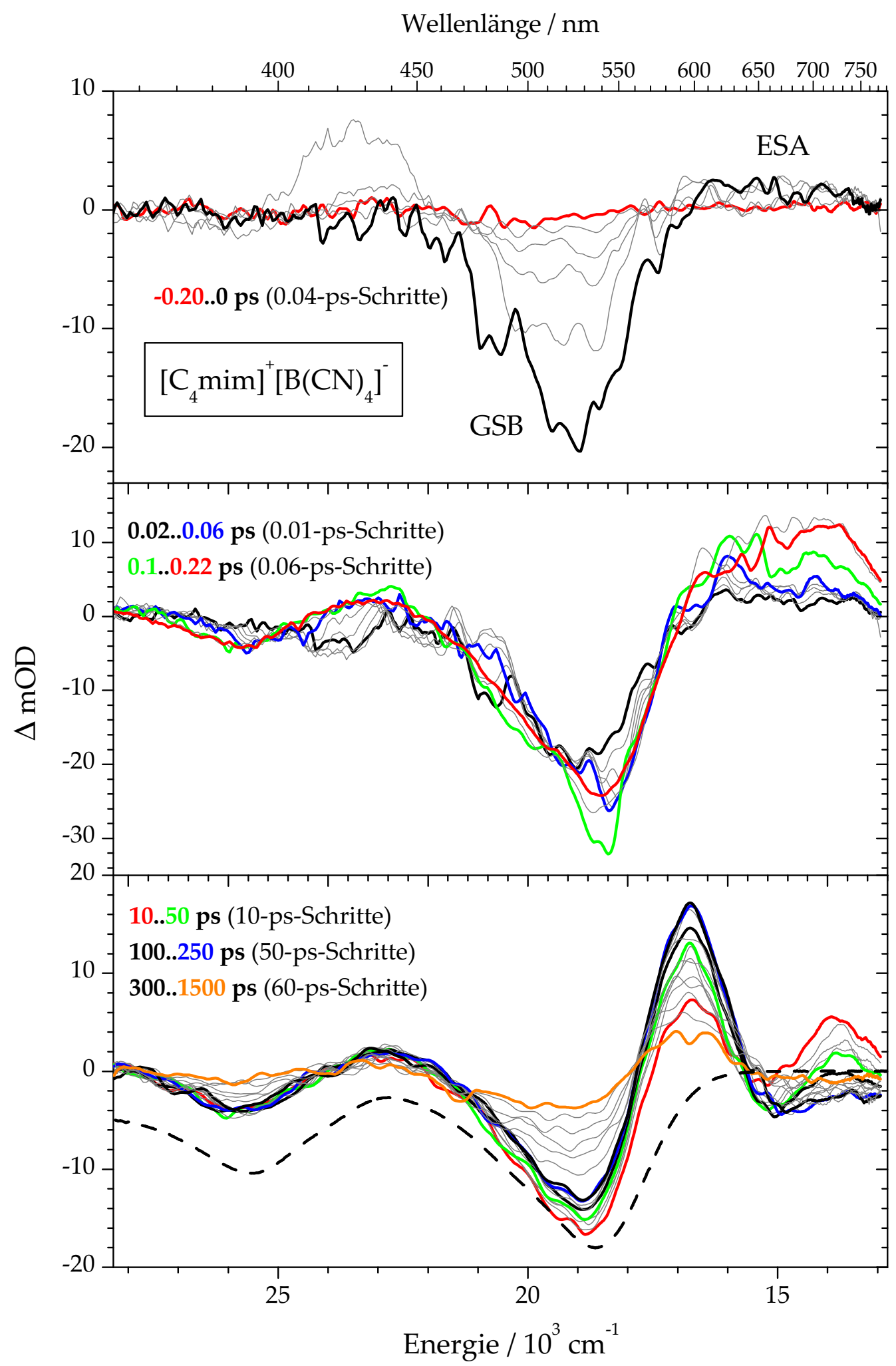

Abbildung 6.11: Transientes PSCP-Absorptionsspektrum von D149 in $\left[\mathrm{C}_{4} \mathrm{mim}\right]^{+}\left[\mathrm{B}(\mathrm{CN})_{4}\right]^{-}$bei einer Anregungswellenlänge von $480 \mathrm{~nm}$. 


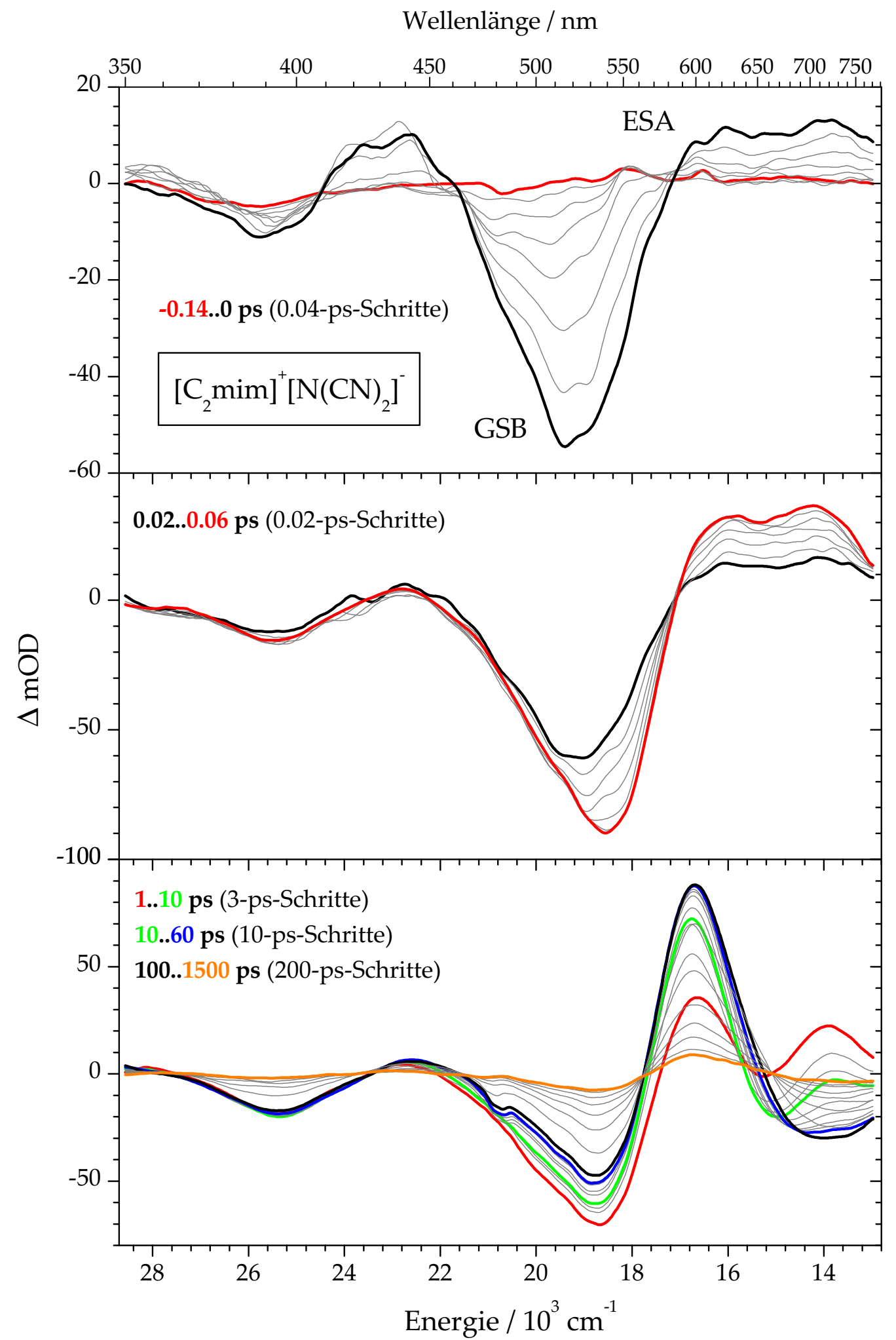

Abbildung 6.12: Transientes PSCP-Absorptionsspektrum von D149 in $\left[\mathrm{C}_{2} \mathrm{mim}\right]^{+}\left[\mathrm{N}(\mathrm{CN})_{2}\right]^{-}$bei einer Anregungswellenlänge von $480 \mathrm{~nm}$. 
Im mittleren und unteren Feld wird eine spektrale Verschiebung der transienten Spektren deutlich. Dieses Phänomen wird ebenfalls bei der Verwendung von Apocarotinoiden als molekulare Sonden beobachtet. Diese Verschiebung ist auf die Solvensrelaxation nach Anregung der Sonde zurückzuführen. Besonders auffällig ist die inertiale Blauverschiebung der größeren ESA-Bande bei 550 bis $670 \mathrm{~nm}$ bei frühen Zeiten. Nach kurzer Zeit wandelt sich diese Blauverschiebung in eine leichte Rotverchiebung um, bis das Absorptionsmaximum konstant bleibt. Dies ist in Abbildung 6.14 verdeutlicht. Hier sind die Absorptionsmaxima dieser ESA-Bande gegen die Zeit aufgetragen. Es resultieren zwei Zeitkonstanten für die schnelle Blauverschiebung $\left(\tau_{s}\right)$ und für die langsamere Rotverschiebung bei späteren Zeiten $\left(\tau_{l}\right)$. Diese Zeitkonstanten sind in Tabelle 6.1 aufgeführt. Die Bande der stimulierten Emission zeigt dem gegenüber eine beginnende Verschiebung ins Rote gefolgt von einer minimalen Blauverschiebung. In der folgenden Abbildung ist ein Vergrößerung der beiden Banden von $\mathrm{D} 149$ in $\left[\mathrm{C}_{2} \mathrm{mim}\right]^{+}\left[\mathrm{N}(\mathrm{CN})_{2}\right]^{-}$gezeigt.

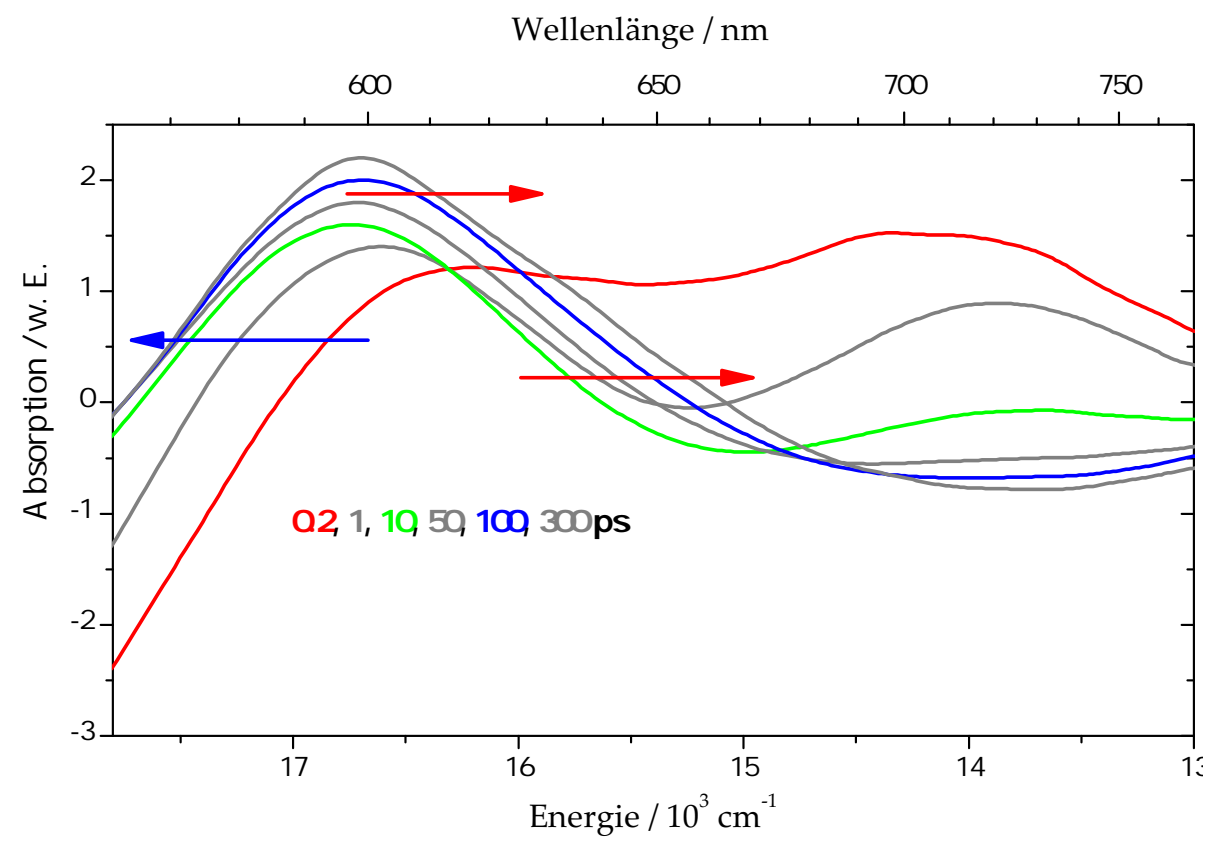

Abbildung 6.13: Ausschnitt der ESA- und SE-Banden von D149 in $\left[\mathrm{C}_{2} \mathrm{mim}\right]^{+}\left[\mathrm{N}(\mathrm{CN})_{2}\right]^{-}$.

Diese spektrale Eigenschaften werden durch eine Solvensrelaxation verursacht. Durch die Relaxation kommt es zu einer schnellen Stabilisierung des $S_{1}$-Zustands relativ zum Grundzustand, die Energielücke wird kleiner, daher schiebt die SE-Bande in den längerwelligen Bereich. Zeitgleich wird die Energielücke zum höher angeregtem Zustand größer, 
woraus die Blauverschiebung der ESA-Bande resultiert. Auf einer längeren Zeitskala tritt ein destabilisierender Effekt auf, der aber keinen so großen Einfluss hat. Hierbei könnte es sich allerdings auch um einen anderen Kanal handeln, wie z. B. eine Isomerisierung der Sonde. Für den Wechsel zwischen dem stabilisierenden und dem destabilisierenden Effekt kann durch eine Anpassung mit drei Exponentialfunktionen eine dritte Zeitkonstante $\left(\tau_{m}\right)$ gewonnen werden, die etwas langsamer ist als $\tau_{s}$ (siehe Abbildung 6.14). Diese Befunde werden ausführlich in Kapitel 6.5 diskutiert.

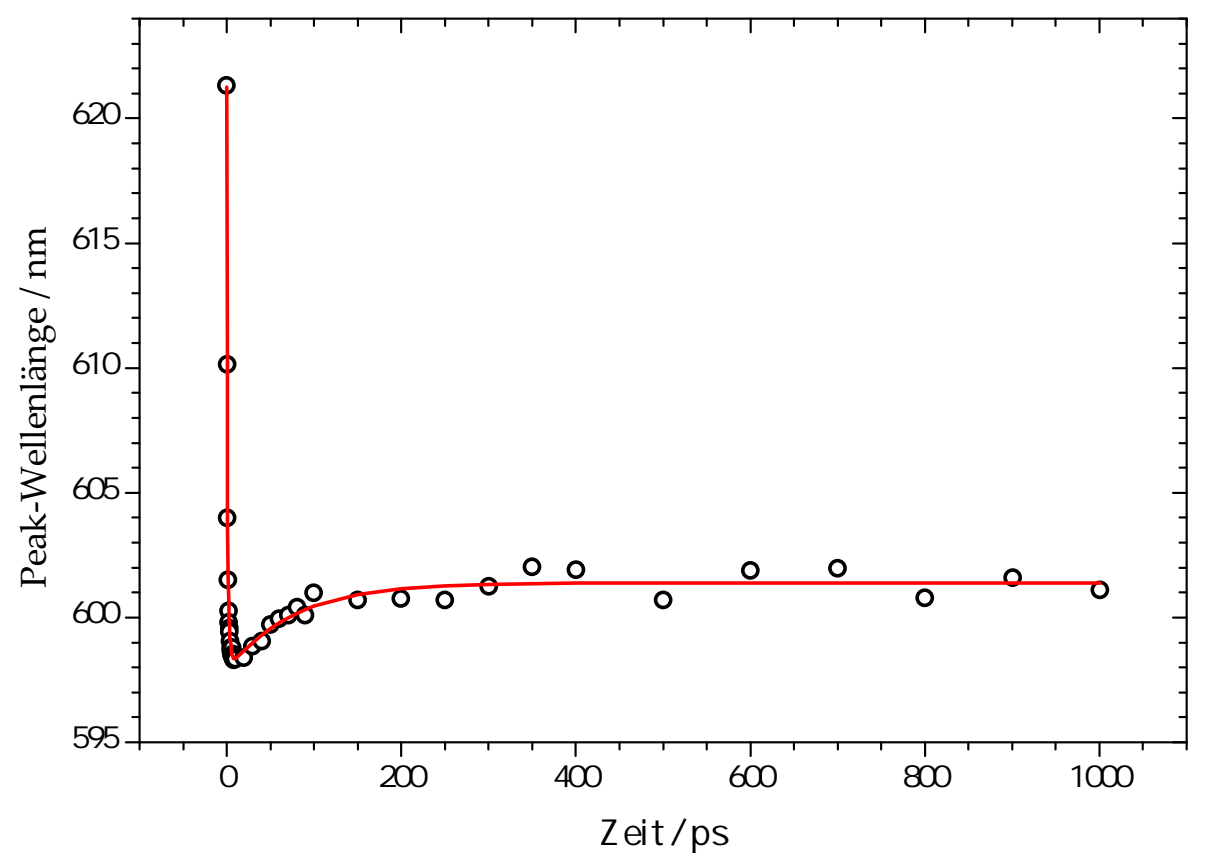

Abbildung 6.14: Transiente Absorptionsmaxima der ESA-Bande von D149 in $\left[\mathrm{C}_{2} \mathrm{mim}\right]^{+}\left[\mathrm{N}(\mathrm{CN})_{2}\right]^{-}$.

Den PSCP-Daten können kinetische Spuren entnommen werden. Die Lebensdauer des $\mathrm{S}_{1}$-Zustands wird unmittelbar von der Polarität des Lösungsmittels beeinflusst. So kann abhängig von der ionischen Flüssigkeit eine globale Analyse der kinetischen Spuren erfolgen (siehe Kapitel 4.2). Dies ermöglicht Schlussfolgerungen auf die Mikopolarität der ionischen Flüssigkeiten. Es werden jeweils exemplarische kinetische Spuren bei unterschiedlichen Wellenlängen in den Abbildungen 6.15 und 6.16 gezeigt. Aufgrund der nicht ganz so guten Qualität der Messung von $\left[\mathrm{C}_{4} \mathrm{mim}\right]^{+}\left[\mathrm{B}(\mathrm{CN})_{4}\right]^{-}$erfolgt hier eine globale Analyse erst ab einer Wellenlänge von etwa $500 \mathrm{~nm}$. Es sind daher nur drei kinetische Spuren gezeigt. Den Abbildungen sind globale Anpassungen der Signale zu entnehmen. Die PSCP-Daten lassen sich am besten durch biexponentielle Funktionen simulieren. Die Zeitkonstanten $\tau_{1, A}$ 
und $\tau_{1, B}$ der Modellierung für die innere Konversion $S_{1} \rightarrow S_{0}$ sind in Tabelle 6.1 zusammengefasst. Die Lebensdauer des $\mathrm{S}_{1}$-Zustands von D149 in $\left[\mathrm{C}_{4} \mathrm{mim}\right]^{+}\left[\mathrm{B}(\mathrm{CN})_{4}\right]^{-}$scheint sehr hoch zu sein und kann aufgrund der begrenzten Zeitverzögerung von Pump- und Pobestrahl nicht komplett gemessen werden, daher sind die angegebenen Zeitkonstanten als relativ ungenau anzusehen.

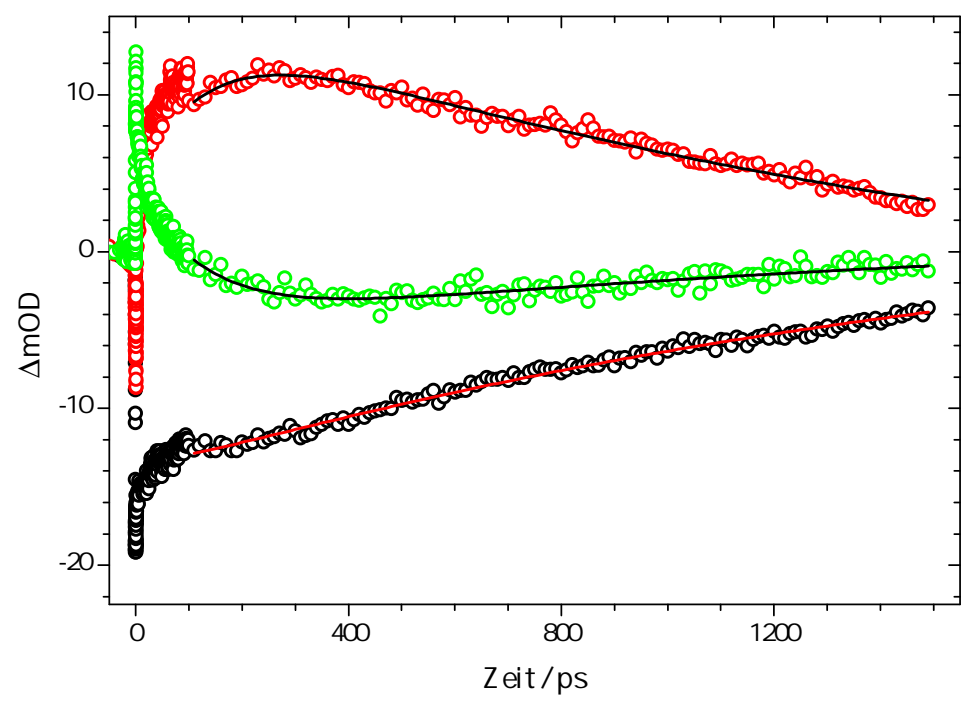

Abbildung 6.15: Kinetische Spuren mit globaler Anpassung von D149 in $\left[\mathrm{C}_{4} \mathrm{mim}\right]^{+}\left[\mathrm{B}(\mathrm{CN})_{4}\right]^{-}$bei vier repräsentativen Wellenlängen. (०) $423 \mathrm{~nm},($ ) $522 \mathrm{~nm}$, ( ) $563 \mathrm{~nm}$ und (०) $620 \mathrm{~nm}$.

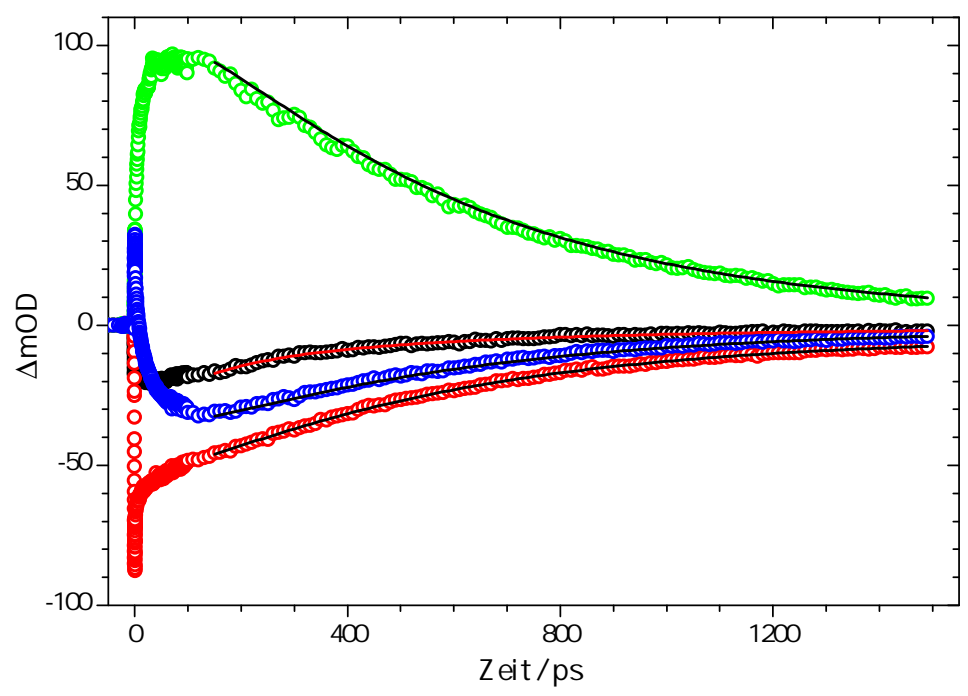

Abbildung 6.16: Kinetische Spuren mit globaler Anpassung von D149 in $\left[\mathrm{C}_{2} \mathrm{mim}\right]^{+}\left[\mathrm{N}(\mathrm{CN})_{2}\right]^{-}$bei vier repräsentativen Wellenlängen. (०) $392 \mathrm{~nm}$, (०) $531 \mathrm{~nm}$, ( () $595 \mathrm{~nm}$ und (०) $734 \mathrm{~nm}$. 
Im Folgenden werden alle ermittelten Zeitkonstanten aus den transienten Absorptionsexperimenten von den idolinbasierten Farbstoffen in organischen Lösungsmitteln und ionischen Flüssigkeiten in einer Tabelle zusammengefasst. Anhand dieser Ergebnisse erfolgt eine Diskussion über die Wechselwirkung der Farbstoffe mit dem Lösungsmittel im nächsten Abschnitt.

Tabelle 6.1: Anpassungsparameter in ps

\begin{tabular}{|c|c|c|c|c|c|c|c|c|}
\hline Lösungsmittel & $\tau_{1}$ & $\tau_{2}$ & $\tau_{3}$ & $\tau_{1, A}$ & $\tau_{1, B}$ & $\tau_{l}$ & $\tau_{s}$ & $\tau_{m}$ \\
\hline \multicolumn{9}{|l|}{ D102 } \\
\hline Acetonitril & 244 & 0.2 & 1.0 & & & & & \\
\hline THF & 301 & 0.8 & 7.6 & & & & & \\
\hline Ethanol & 133 & 0.9 & 19.7 & & & & & \\
\hline Methanol & 56 & 0.6 & 7.1 & & & & & \\
\hline \multicolumn{9}{|l|}{ D131 } \\
\hline Acetonitril & 288 & 0.7 & 8.0 & & & & & \\
\hline THF & 666 & 1.4 & 11.2 & & & & & \\
\hline Ethanol & 136 & 0.4 & 29.5 & & & & & \\
\hline Methanol & 73 & 1.2 & 12.8 & & & & & \\
\hline \multicolumn{9}{|l|}{ D149 } \\
\hline Acetonitril & 239 & 0.6 & 1.5 & & & & & \\
\hline THF & 828 & 2.6 & 33.8 & & & & & \\
\hline Ethanol & 185 & 3.0 & 21.9 & & & & & \\
\hline Methanol & 70 & 1.1 & 7.4 & & & & & \\
\hline$\left[\mathrm{C}_{4} \mathrm{mim}\right]^{+}\left[\mathrm{B}(\mathrm{CN})_{4}\right]^{-}$ & & & & 1670 & 127 & & 8 & \\
\hline$\left[\mathrm{C}_{2} \mathrm{mim}\right]^{+}\left[\mathrm{N}(\mathrm{CN})_{2}\right]^{-}$ & & & & 516 & 131 & 75 & 0.4 & 2.3 \\
\hline \multicolumn{9}{|l|}{ D205 } \\
\hline Acetonitril & 251 & 0.2 & 0.8 & & & & & \\
\hline THF & 673 & 2.7 & 36.6 & & & & & \\
\hline Ethanol & 308 & 4.4 & 30.5 & & & & & \\
\hline Methanol & 104 & 1.4 & 9.2 & & & & & \\
\hline
\end{tabular}




\subsection{Diskussion}

Die elektronisch angeregten Zustände der indolinbasierten Farbstoffe D102, D131, D149 und D205 zeigen ein interessantes Verhalten in orgnischen Lösungsmitteln. Mit Hilfe der transienten Absorptionsspektroskopie sind die Lebensdauern des ersten angeregten $\mathrm{Zu}$ stands $\mathrm{S}_{1}$ bestimmt worden. Diese sind in Tabelle 6.1 aufgeführt. Für eine detaillierte Übersicht der Verläufe dieser Lebensdauern in Abhängigkeit vom Lösungsmittel sei auf die nachfolgende Abbildung verwiesen. Die Lebensdauer $\tau_{1}$ folgt hierbei folgenden Trend: THF > Acetonitril > Ethanol > Methanol. Eine Ausnahme bildet der Farbstoff D205, hier ist $\tau_{1}$ in Ethanol größer als in Acetonitril.
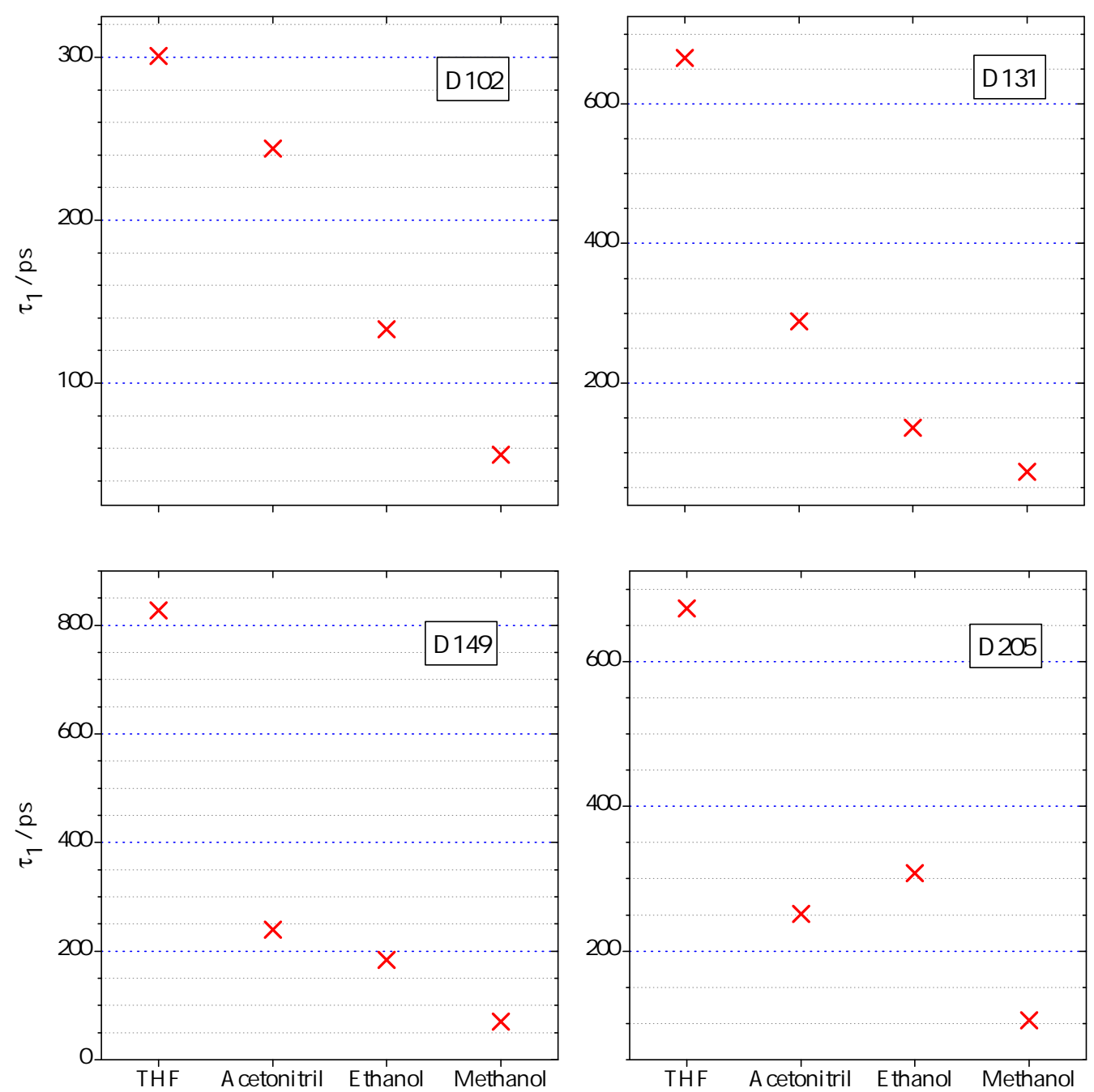

Abbildung 6.17: Vergleich der Lebensdauern $\tau_{1}$ der indolinbasierten Farbstoffe in verschiedenen organischen Lösungsmitteln. 
Die Polarität des Lösungsmittels scheint nicht der einzige Beitrag zur Stabilisierung bzw. Destabilisierung des $S_{1}$-Zustands relativ zum Grundzustand zu sein. Obwohl Methanol und Acetonitril eine ähnliche Polarität aufweisen, unterscheiden sich $\tau_{1}$ aller Farbstoffe in diesen Lösungsmitteln beträchtlich. Eine mögliche Erklärung hierfür könnte die Ausbildung von Wasserstoffbrücken sein. Diese Art von Wechselwirkung, die in Ethanol und Methanol auftreten würde, könnte einen zusätzlichen stabilisierenden Effekt hervorrufen, was zu einer Abnahme der Zeitkonstanten führen würde. Aufgrund einer höheren sterischen Hinderung von D205 verglichen mit D149 nimmt die stabilisierende Wirkung der Wasserstoffbrücken bei diesem Farbstoff ab, hier sind die Zeitkonstanten in Ethanol und Methanol am höchsten. D205 besitzt einen Octylrest an der Rhodanineinheit im Gegensatz zu D149, welches einen Ethylrest hat. Die höchste Stabiliesierung des $\mathrm{S}_{1}$-Zustands ist bei D102 dem kleinsten der Moleküle zu finden.

Weitere Einblicke in die mikroskopische Wechselwirkung der Farbstoffe mit dem Lösungsmittel erhält man durch das Betrachten der Lebensdauer $\tau_{3}$. Hierbei handelt es sich um eine Dynamik bei der Bildung des $S_{1}$-Zustands die durch intertiale Bewegung der Solvensmoleküle hervorgerufen wird. Es folgt ein Vergleich dieser Zeitkonstante analog zu $\tau_{1}$. Durch diese Abbildung gewinnt man den Eindruck, dass das Verhalten von D102 und D131 sowie D149 und D205 sich jeweils ähnelt. Im Fall von D102 und D131 sind die $\tau_{3^{-}}$ Werte von THF und Methanol etwa gleich, wohingegen sie bei D149 und D205 in THF sehr viel größer sind. Wie in den TA-Signalen zu erkennen (siehe Abbildungen 6.7-6.10), unterscheiden sich die Messungen in THF bei allen Farbstoffen von den anderen Lösungsmitteln. Die Signale haben in THF eine gänzlich andere Form. Daher sind besonders die $\tau_{3}$-Werte von THF mit Vorsicht zu genießen. Es bleibt die Diskussion der Zeitkonstanten der Solvensrelaxation in den übrigen Lösungsmitteln.

In den Lösungsmitteln Acetonitril, Ethanol und Methanol zeigen alle gemessenen Farbstoffe bezüglich des Einflusses der Solvensrelaxation ein gleiches Verhalten. Die ermittelten $\tau_{3}$-Werte sind in Acetonitril am niedrigsten gefolgt von Methanol und dann Ethanol. Dieser Trend wird ebenfalls bei der Wechselwirkung mit Apocarotinoiden gefunden, wie in früherer Arbeit gezeigt werden konnte. ${ }^{80}$ Die Rotation der Lösungsmittelmoleküle nach Anregung der molekularen Sonden könnte eine Erklärung für diese zusätzliche Zeitkonstante sein. Dies würde auch Sinn machen, denn das kleinste Molekül Acetonitril, welches 
sicherlich am leichtesten rotieren kann, liefert auch die kleinste Zeitkonstante.
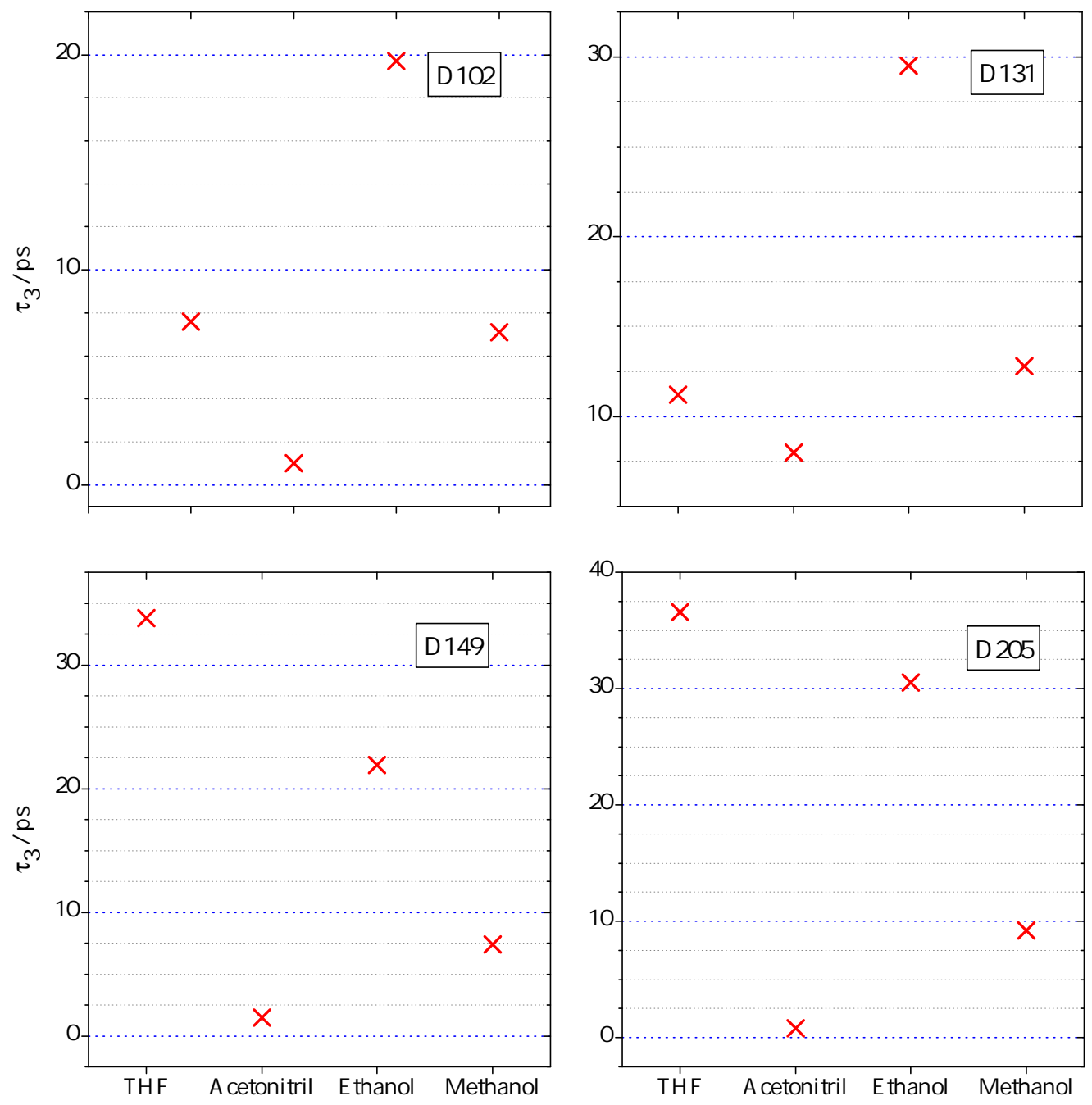

Abbildung 6.18: Vergleich der Lebensdauern $\tau_{3}$ der indolinbasierten Farbstoffe in verschiedenen organischen Lösungsmitteln.

Die abweichende Form der transienten Signale der Farbstoffe in THF lässt sich durch Betrachtung der PSCP-Spektren von D149 in den organischen Lösungsmitteln erklären. Wie in Abbildung 6.19 zu sehen, tritt im Fall von THF auch bei langen Zeiten keine beobachtbare stimulierte Emission im nahen IR-Bereich auf, diese könnte allerdings von einer transienten Absorption überlagert sein. Hier bleibt eine ESA erhalten, daher beobachtet man im Zwei-Farben-Experiment ein Abklingen der transienten Absorption vom $\mathrm{S}_{1}$-Zustand in höhere Niveaus. In der nachfolgenden Abbildung sind die PSCP-Spektren von D149 in Acetonitril, Methanol und THF miteinander verglichen, sie sind jeweils normiert zum 
Zeitpunkt von $\tau_{1}$ gezeigt. Bei dem Spektrum von THF musste im Bereich von 450 und $500 \mathrm{~nm}$ eine Streulichtschwankung abgezogen werden. Die Daten sind aus früherer Arbeit übernommen worden. 128

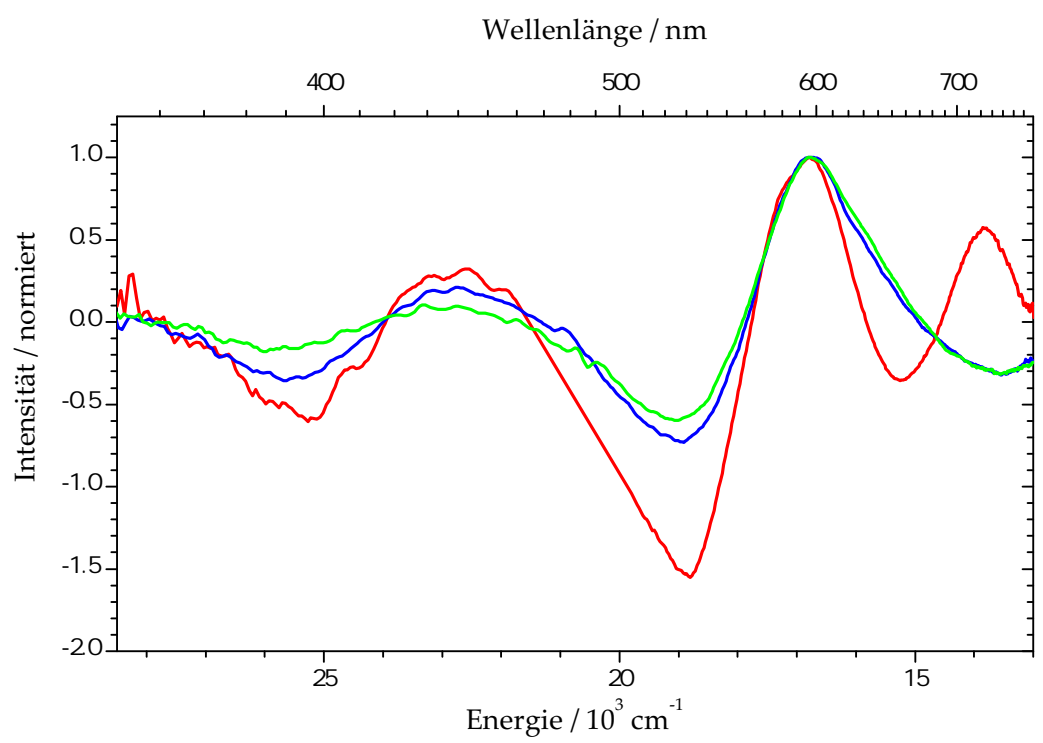

Abbildung 6.19: Vergleich der PSCP-Spektren von D149 in organischen Lösungsmitteln. — THF, Methanol und - Acetonitril.

Zusammenfassend lässt sich nocheinmal feststellen, dass die Zeitkonstante $\tau_{1}$ bei den Messungen in der Reihenfolge D205 $\rightarrow$ D149 $\rightarrow$ D131 $\rightarrow$ D102 abnimmt. Dies trifft zumindest $\mathrm{zu}$, betrachtet man die Messungen in Ethanol und Methanol. Diese vier Farbstoffe sind bereits im Hinblick auf ihren Wirkungsgrad in Farbstoffsolarzellen untersucht worden. 125:132 Es konnte festgestellt werden, dass Indolinsensibilisator D205 den höchsten Wirkungsgrad erzielt. Es sind vergleichende Messungen von D205, D149, D131 und D102 mit organischen Lösungsmitteln als Elektrolyte gemacht worden. Die Reihenfolge der Wirkungsgrade nimmt mit kleiner werdenden Molekülgröße ab. Die korrespondiert mit der Lebensdauer des $S_{1}$-Zustands dieser Farbstoffe beispielsweise in Ethanol. Der Farbstoff D205 mit dem höchsten Wirkungsgrad in DSSCs zeigt die höchste Lebensdauer. Bei den anderen Farbstoffen nimmt die Lebensdauer ab und auch der Wirkungsgrad wird geringer. Eine mögliche Erklärung wär, dass die innere Konversion $S_{1} \rightarrow S_{0}$ immer stärker in Konkurrenz zur Elektroneninjektion in den Halbleiter tritt. Natürlich müssten zur Verifizierung dieser These transiente Messungen der Farbstoffe auf einer Halbleiteroberfläche durchgeführt werden.

Wie in der Einleitung erwähnt, ist es wünschenswert, organische Lösungsmittel als Elek- 
trolyte in den DSSCs zu ersetzen. Der große Nachteil dieser Elektrolyte ist deren starke Verdampfung, was zu einer geringeren Haltbarkeit der DSSCs führen könnte. Einen möglichen Ersatz könnten die ionischen Flüssigkeiten bieten. In dieser Arbeit ist der Farbstoff D149 in den ionischen Flüssigkeiten $\left[\mathrm{C}_{2} \mathrm{mim}\right]^{+}\left[\mathrm{N}(\mathrm{CN})_{2}\right]^{-}$und $\left[\mathrm{C}_{4} \mathrm{mim}\right]^{+}\left[\mathrm{B}(\mathrm{CN})_{4}\right]^{-}$untersucht worden. Es liegen interessante Ergebnisse der Solvatationsdynamik der indolinbasierten Sonde in den ILs vor. So ergibt sich ein gänzlich anderes Solvatationsverhalten als bei den gemessenen Carotinoiden. Bei der biexponentiellen Anpassung der kinetischen Spuren ergibt sich eine langsame $\left(\tau_{1, A}\right)$ und eine etwas schnellere Komponente $\left(\tau_{1, A}\right)$. Die schnelle Zeitkonstante ist in beiden ionischen Flüssigkeiten in etwa gleich. Die langsame Zeitkonstante zeigt einen großen Unterschied. Der Unterschied der beiden Lebensdauern von D149 in $\left[\mathrm{C}_{2} \mathrm{mim}\right]^{+}\left[\mathrm{N}(\mathrm{CN})_{2}\right]^{-}$und $\left[\mathrm{C}_{4} \mathrm{mim}\right]^{+}\left[\mathrm{B}(\mathrm{CN})_{4}\right]^{-}$beträgt ca. $1000 \mathrm{ps}$, wobei es in $\left[\mathrm{C}_{2} \mathrm{mim}\right]^{+}\left[\mathrm{N}(\mathrm{CN})_{2}\right]^{-}$eine geringere Lebensdauer gibt. Dies ist ein enormer Unterschied, der so bei der Verwendung von Carotinoiden als molekulare Sonden nicht gefunden werden konnte. Dies zeichnet D149 als eine sehr mächtige Sonde aus, mit der man wahrscheinlich durch transiente Absorptionsspektroskopie die Mikropolarität von ionischen Flüssigkeiten sehr gut erkunden kann. Durch ein solch großes Zeitfenster lassen sich kleine Unterschiede bei der Solvatation leichter erfassen. Es konnte noch nicht hinreichend geklärt werden, ob es sich bei der Dynamik um eine Isomersierung handelt, daher sollten in Zukunft weitere Untersuchungen mit dieser Sonde durchgeführt werden.

Bei diesem starken Effekt handelt es sich wohl um die Wechselwirkung mit dem Anion der ionischen Flüssigkeit. Das Kation der beiden ionischen Flüssigkeiten unterscheidet sich nur geringfügig in der Alkylkettenlänge, jedoch ist das Anion völlig unterschiedlich. Die Wechselwirkung mit dem Tetracyanoboratanion scheint eine eine stark destabilisierende Wirkung auf den ersten angeregten Zustand zu haben. Interessant ist die Relaxationsdynamik beider ionischen Flüssigkeiten, die sich in den PSCP-Messungen als spektrale Verschiebung der transienten Spektren bemerkbar macht. Wird die ESA-Bande bei 550 bis 640 $\mathrm{nm}$ betrachtet, so ergibt sich bei kurzen Zeiten eine Blauverschiebung die mit $\tau_{s}$ abklingt. Dem folgt eine leichte Rotverschiebung auf einer längeren Zeitskala $\tau_{l}$. Der Wechsel beider Verschiebungen erfolgt mit $\tau_{m}$. Diese drei Zeitkonstanten konnten nur für $\left[\mathrm{C}_{2} \mathrm{mim}\right]^{+}$$\left[\mathrm{N}(\mathrm{CN})_{2}\right]^{-}$aufgelöst werden. Bei $\left[\mathrm{C}_{4} \mathrm{mim}\right]^{+}\left[\mathrm{B}(\mathrm{CN})_{4}\right]^{-}$ist dies aufgrund von apparativ bedingten Schwankungen nicht möglich. Hier konnte allerdings die Dauer für die schnel- 
le Blauverschiebung extrahiert werden. Ein Vergleich der beiden ionischen Flüssigkeiten zeigt, dass auch die Blauverschiebung von D149 in $\left[\mathrm{C}_{4} \mathrm{mim}\right]^{+}\left[\mathrm{B}(\mathrm{CN})_{4}\right]^{-}$länger dauert.

Die spektrale Verschiebung in den kürzerwelligen Bereich im Fall der ESA-Banden bedeutet, dass die Energiedifferenz zwischen $S_{1}$ und dem höher gelegenen Niveau $S_{n}$ größer wird. Im Umkehrschluss bedeutet dies eine Stabilisierung von $\mathrm{S}_{1}$ relativ zum Grundniveau, denn die stimulierte Emissionsbande schiebt gleichzeitig ins Rote. Dieser Prozess läuft sehr schnell ab. Hierbei könnte es sich um eine inertiale Bewegung der Kationen handeln, die sich nach der Anregung von D149 neu ausrichten. Im Fall dieser indolinbasierten molekularen Sonde tritt ein weiterer Effekt auf, der bei den Carotinoiden in der Form nicht beobachtet werden konnte. Nach dieser schnelle Stabilisierung erfolgt eine relativ schwache Destabilisierung auf einer längeren Zeitskale. Dies könnte durch repulsive Wechselwirkungen der Anionen mit dem negativen Ende des Charge-Transfer-Zustands hervorgerufen werden. Dieser Effekt ist wie gesagt aber sehr klein.

Ionische Flüssigkeiten eignen sich als Elektrolyte für Farbstoffsolarzellen. Als besonders effizient haben sich auf Tetracyanoborat basierende ionische Flüssigkeiten herausgestellt. Es gibt noch keine ultraschnelle spektroskopische Untersuchungen der indolinbasierten Farbstoffe auf einer Halbleiterschicht gekoppelt mit dem Einfluss der ionischen Flüssigkeit. Doch lassen die Ergebnisse dieser Arbeit vermuten, dass es einen starken Zusammenhang zwischen der Wechselwirkung mit dem Elektrolyten und der Effizienz der Solarzellen gibt. Es konnte gezeigt werden, dass der angeregt Zustand von D149 eine sehr große Lebensdauer in der tetracyanoboratbasierten ionischen Flüssigkeit besitzt. Dies könnte wiederum bedeuten, dass durch die Wahl des Elektrolyten Konkurrezprozesse zur Elektroneninjektion unterdrückt werden können. 


\section{Literaturverzeichnis}

[1] Weingärtner, H. Angew. Chem. Int. Ed. 2008, 47, 654.

[2] Wasserscheid, P.; Welton, T. Ionic Liquids in Synthesis; Wiley-VCH:Weinheim, 2007.

[3] Endres, F.; El-Abedin, S. Z. Phys. Chem. Chem. Phys. 2006, 8, 2101.

[4] Holbrey, J. D.; Seddon, K. R. J. Chem. Soc. Dalton 1999, 8, 2133.

[5] Crosthwaite, J. M.; Muldoon, M. J.; Dixon, J. K.; Anderson, J. L.; Brennecke, J. F. J. Chem. Thermo. 2005, 37, 559.

[6] Earle, M. J.; Esperança, J. M. S. S.; Gilea, M. A.; Canongie-Lopes, J. N.; Rebelo, L. P. N.; Magee, J. W.; Seddon, K. R.; Widegren, J. A. Nature (London) 2006, 439, 831.

[7] Rebelo, L. P. N.; Canongie-Lopes, J. N.; Esperança, J. M. S. S.; Filipe, E. J. Phys. Chem. B 2005, 109, 6040 .

[8] Ngo, H. L.; LeCompte, K.; Hargens, L.; McEwen, A. B. Thermochim. Acta 2000, 357, 97.

[9] Fox, D. M.; Gilman, J. W.; DeLong, H. C.; Trulove, P. C. J. Chem. Thermo. 2005, 37, 900.

[10] Lohse, P. W. Laserspektroskopische Untersuchungen inter- und intramolekularer Relaxationsprozesse großer organischer Moleküle; Diplomarbeit, Göttingen, 2007.

[11] Tokuda, H.; Tsuzuki, S.; Susan, M. A. B. H.; Hayamizu, K.; Watanabe, M. J. Phys. Chem. B. 2006, 110, 19593.

[12] Tokuda, H.; Hayamizu, K.; Ishii, K.; Susan, M. A. B. H.; Watanabe, M. J. Phys. Chem. B 2005, 109, 6103.

[13] Hyun, B. R.; Dzyuba, S. V.; Bartsch, R. A.; Quitevis, E. L. J. Phys. Chem. A 2002, 106, 7579 .

[14] Giraud, G.; Gordon, C. M.; Dunkin, I. R.; Wynne, K. J. Chem. Phys. 2003, 119, 464.

[15] Cang, H.; Li, J.; Fayer, M. D. J. Chem. Phys. 2003, 119, 13017.

[16] Rajian, J. R.; Li, S.; Bartsch, R. A.; Quitevis, E. L. Chem. Phys. Lett. 2004, 393, 372.

[17] Shirota, H.; Funston, A. M.; Wishart, J. F.; Castner-Jr., E. E. J. Chem. Phys. 2005, 122, 184512.

[18] Xiao, D.; Rajian, J. R.; Cady, A.; Li, S.; Bartsch, R. A.; Quitevis, E. L. J. Phys. Chem. B 2007, 111, 4669. 
[19] Asaki, M. L. T.; Redondo, A.; Zawodzinski, T. A.; Taylor, A. J. J. Chem. Phys. 2002, 116, 10377.

[20] Yamamoto, K.; Tani, M.; Hangyo, M. J. Phys. Chem. B. 2007, 111, 4854.

[21] Triolo, A.; Russina, O.; Arrighi, V.; Juranyi, F.; Janssen, S.; Gordon, C. M. J. Chem. Phys. $2003,119,8549$.

[22] Triolo, A.; Russina, O.; Hardacre, C.; Nieuwenhuyzen, M.; Gonzales, M. A.; Grimm, H. J. Phys. Chem. B 2005, 109, 22061.

[23] Wishart, J. F.; Neta, P. J. Phys. Chem. B 2003, 107, 7261.

[24] Wishart, J. F.; Lall-Ramnarine, S. I.; Raju, R.; Scumpia, A.; Bellevue, S.; Ragbir, R.; Engel, R. Rad. Phys. Chem. 2005, 72, 99.

[25] Brands, H.; Chandrasekhar, N.; Unterreiner, A. N. J. Phys. Chem. B 2007, 111, 4830.

[26] Strehmel, V.; Laschewsky, A.; Stoesser, R.; Zehl, A.; Herrmann, W. J. Phys. Org. Chem. 2006, 19, 318.

[27] Stoesser, R.; Herrmann, W.; Zehl, A.; Laschewsky, A.; Strehmel, V. Z. Phys. Chem. 2006, 220, 1309.

[28] Oehlke, A.; Hofmann, K.; Spange, S. New J. Chem. 2006, 30, 5331698.

[29] Reichardt, C. Green Chem. 2005, 7, 339.

[30] Arzhantsev, S.; Hui, J.; Baker, G. A.; Maroncelli, M. J. Phys. Chem. B 2007, 111, 4978.

[31] Samanta, A. J. Phys. Chem. B 2006, 110, 13704.

[32] Chandrasekhar, N.; Unterreiner, A. N. Phys. Chem. Chem. Phys. 2010, 12, 1698.

[33] Lohse, P. W.; Bürsing, R.; Lenzer, T.; Oum, K. J. Phys. Chem. B 2008, 112, 3048.

[34] O’Regan, B.; Grätzel, M. Nature 1991, 353, 737.

[35] Kawano, R.; Katakabe, T.; Shimosawa, H.; Nazeeruddin, M. K.; Grätzel, M.; Matsui, H.; Kitamura, T.; Tanabec, N.; Watanabe, M. Phys. Chem. Chem. Phys. 2010, 12, 1916.

[36] Lide, D. R. Handbook of Chemistry and Physics; CRC Press, 1995-1996.

[37] Strehmel, V.; Rexhausen, H.; Strauch, P. Tetrahedron Lett. 2008, 49, 586.

[38] Muller, P. Pure Appl. Chem. 1994, 66, 409.

[39] Reichardt, C.; Harbusch-Görnert, E. Liebigs Ann. Chem. 1983, 721.

[40] Poole, C. F. J. Chromatogr. A 2004, 1037, 49.

[41] Abboud, J. L. M.; Kamlet, M. J.; Taft, R. W. Progress in Physical Organic Chemistry; John Wiley \& Sons, 1981. 
[42] Laurence, C.; Nicolet, P.; Dalati, M. T.; Abboud, J. L. M.; Notario, R. J. Chem. Phys. $1994,98,5807$.

[43] Harrod, W. B.; Pienta, N. J. J. Phys. Org. Chem. 1990, 3, 534.

[44] Muldoon, M. J.; Gordon, C. M.; Dunkin, I. R. J. Chem. Soc. Perkin Trans. 2001, 2, 433.

[45] Baker, S. N.; Baker, G. A.; Bright, F. V. Green Chem. 2002, 4, 165.

[46] Fletcher, K. A.; Pandey, S. Proc. Electrochem. Soc. 2002, 19, 244.

[47] Fletcher, K. A.; Baker, S. N.; Baker, G. A.; Pandey, S. New. J. Chem. 2003, 27, 1706.

[48] Fredlake, C. P.; Muldoon, M. J.; Aki, S. N. V. K.; Welton, T.; Brennecke, J. F. Phys. Chem. Chem. Phys. 2004, 6, 3280.

[49] Crowhurst, L.; Mawdsley, P. R.; Perez-Arlandis, J. M.; Salter, P. A.; Welton, T. Phys. Chem. Chem. Phys. 2003, 5, 2790.

[50] Taft, R. W.; Pienta, N. J.; Kamlet, M. J.; Arnett, E. M. J. Org. Chem. 1981, 661, 46.

[51] Stoesser, R.; Herrmann, W.; Zehl, A.; Strehmel, V.; Laschewsky, A. ChemPhysChem 2006, 7, 1106.

[52] Stoesser, R.; Herrmann, W.; Zehl, A.; Laschewsky, A.; Strehmel, V. Z. Phys. Chem. 2006, 220, 1309.

[53] Strehmel, V.; Rexhausen, H.; Strauch, P. Tetrahedron Lett. 2008, 49, 3264.

[54] Strehmel, V.; Rexhausen, H.; Strauch, P. Tetrahedron Lett. 2008, 49, 7143.

[55] Strehmel, V.; Rexhausen, H.; Strauch, P.; Strehmel, B. ChemPhysChem 2008, 9, 1294.

[56] Strehmel, V.; Rexhausen, H.; Strauchl, P. Phys. Chem. Chem. Phys. 2010, 12, 1933.

[57] Bart, E.; Huppert, D. Chem. Phys. Lett. 1992, 195, 37.

[58] Bart, E.; Meltsin, A.; Huppert, D. Chem. Phys. Lett. 1992, 200, 592.

[59] Bart, E.; Meltsin, A.; Huppert, D. J. Phys. Chem. 1994, 98, 3295.

[60] Bart, E.; Meltsin, A.; Huppert, D. J. Phys. Chem. 1994, 98, 10819.

[61] Karmakar, R.; Samanta, A. J. Phys. Chem. A 2002, 106, 4447.

[62] Karmakar, R.; Samanta, A. J. Phys. Chem. A 2002, 106, 6670.

[63] Karmakar, R.; Samanta, A. J. Phys. Chem. A 2003, 107, 7340.

[64] Saha, S.; Mandal, P. K.; Samanta, A. Phys. Chem. Chem. Phys. 2004, 6, 3106.

[65] Mandal, P. K.; Paul, A.; Samanta, A. Res. Chem. Intermed. 2005, 31, 575.

[66] Mandal, P. K.; Samanta, A. J. Phys. Chem. B 2005, 109, 15172.

[67] Samanta, A. J. Phys. Chem. Lett. 2010, 1, 1557. 
[68] Chakraborty, A.; Seth, D.; Chakrabarty, D.; Setua, P.; Sarkar, N. J. Phys. Chem. A 2005, $109,11110$.

[69] Halder, M.; Headley, L. S.; Mukherjee, P.; Song, X.; Petrich, J. W. J. Phys. Chem. A 2006, $110,8623$.

[70] Baker, S. N.; Baker, G. A.; Munson, C. A.; Bukowski, E. J.; Cartwright, A. N.; Bright, F. V. Ind. Eng. Chem. Res. 2003, 42, 6457.

[71] Lang, B.; Angulo, G.; Vauthey, E. J. Phys. Chem. A 2006, 110, 7028.

[72] Ingram, J.; Moog, R. S.; Ito, N.; Biswas, R.; Maroncelli, M. J. Phys. Chem. B 2003, 107, 5926.

[73] Arzthantsev, S.; Ito, N.; Heitz, M.; Maroncelli, M. Chem. Phys. Lett. 2003, 381, 278.

[74] Ito, N.; Arzthantsev, S.; Heitz, M.; Maroncelli, M. J. Phys. Chem. B 2004, 108, 5771.

[75] Ito, N.; Arzthantsev, S.; Maroncelli, M. Chem. Phys. Lett. 2004, 396, 83.

[76] Arzhantsev, S.; Jin, H.; Ito, N.; Maroncelli, M. Chem. Phys. Lett. 2006, 417, 524.

[77] Kovalenko, S. A.; Dobryakov, A. L.; Ruthmann, J.; Ernsting, N. P. Phys. Rev. A 1999, $59,2369$.

[78] Lenzer, T.; Schubert, S.; Ehlers, F.; Lohse, P. W.; Scholz, M.; Oum, K. Archives of Biochemistry and Biophysics 2009, 483, 213.

[79] Wild, D. A.; Winkler, K.; Stalke, S.; Oum, K.; Lenzer, T. Phys. Chem. Chem. Phys. 2006, $8,2499$.

[80] Kopczynski, M.; Ehlers, F.; Lenzer, T.; Oum, K. J. Phys. Chem. A 2007, 111, 5370.

[81] Stalke, S.; Wild, D. A.; Lenzer, T.; Kopczynski, M.; Lohse, P. W.; Oum, K. Phys. Chem. Chem. Phys. 2008, 10, 2180.

[82] Oum, K.; Lohse, P. W.; Ehlers, F.; Scholz, M.; Kopczynski, M.; Lenzer, T. Angew. Chem. Int. Ed. 2010, 49, 2230.

[83] Lohse, P. W.; Ehlers, F.; Oum, K.; Scholz, M.; Lenzer, T. Chem. Phys. 2010, 373, 45.

[84] Demtröder, W. Laserspektroskopie, Grundlagen und Techniken; Springer, 2007.

[85] Kappel, C. Stoßwellenuntersuchungen zur Kinetik und Druckabhängigkeit der Wasserstoffperoxidpyrolyse mittels Laser Absorptions-Spektroskopie; Doktorarbeit, Georg-AugustUniversität Göttingen, 2002.

[86] Lustres, J. L. P.; Kovalenko, S. A.; Mosquera, M.; Senyushkina, T.; Flasche, W.; Ernsting, N. P. Angew. Chem. Int. Ed. 2005, 44, 5635.

[87] von Benten, R. Der Einfluss von chemischer Konstitution, Symmetrie und molekularer Umgebung auf die intramolekulare Schwingungsrelaxation aromatischer Moleküle; Doktorarbeit, Georg-August-Universität Göttingen, 2005. 
[88] Ye, J.; Cundiff, S. T. Femtosecond Optical Frequency Comb Technology: Principle, Operation and Application; Springer, 2005.

[89] Niedrig, H. Lehrbuch der Experimentalphysik, Optik, Bd. 3; Gruyter, 2004.

[90] Nibbering, E. T. J.; Dühr, O.; Korn, G. Opt. Lett. 1997, 22, 1335.

[91] Knox, W. H.; Fork, R. L.; Downer, M. C.; Stolen, R. H.; Shank, C. V.; Valdmanis, J. A. Appl. Phys. Lett. 1985, 46, 1120.

[92] Fork, R. L.; Shank, C. V.; Hirlimann, C.; Yen, R.; Tomlinson, W. J. Opt. Lett. 1983, 8, 1.

[93] Paul, H. Lexikon der Optik; Spektrum, 2003.

[94] Polívka, T.; Sundström, V. Chem. Rev. 2004, 104, 2021.

[95] Chábera, P.; Fucimann, M.; Hříbek, P.; Polívka, T. Phys. Chem. Chem. Phys. 2009, 11, 8795.

[96] Papagianakis, E.; van Stokkum, I. H. M.; Vengris, M.; Cogdell, R. J.; van Grondelle, R.; Larsen, D. S. J. Phys. Chem. B 2006, 110, 5727.

[97] Zigmantas, D.; Hiller, R. G.; Sharples, F. P.; Frank, H. A.; Sundström, V.; Polívka, T. Phys. Chem. Chem. Phys. 2004, 6, 3009.

[98] He, Z.; Gosztola, D.; Deng, Y.; Gao, G.; Wasielewski, M. R.; Kispert, L. D. J. Phys. Chem. B 2000, 104, 6668.

[99] Ehlers, F.; Lenzer, T.; Oum, K.; Seehusen, J.; Wild, D. A. J. Phys. Chem. A 2007, 111, 2257.

[100] Zigmantas, D.; Hiller, R. G.; Yartsev, A.; Sundström, V.; Polívka, T. J. Phys. Chem. B 2003, 107, 5339 .

[101] Shima, S.; Ilagan, R. P.; Gillespie, N.; Sommer, B. J.; Hiller, R. G.; Sharples, F. P.; Frank, H. A.; Birge, R. R. J. Phys. Chem. A 2003, 107, 8052.

[102] Vaswani, H. M.; Hsu, C.-P.; Head-Gordon, M.; Fleming, G. R. J. Phys. Chem. B 2003, $107,7940$.

[103] Papagianakis, E.; Larsen, D. S.; van Stokkum, I. H. M.; Vengris, M.; Hiller, R. G.; van Grondelle, R. Biochemistry 2004, 43, 15303.

[104] Papagianakis, E.; Vengris, M.; Larsen, D. S.; van Stokkum, I. H. M.; Hiller, R. G.; van Grondelle, R. J. Phys. Chem. B 2006, 110, 512.

[105] Archer, D. G.; Widegren, J. A.; Kirklin, D. A.; Magee, J. W. J. Chem. Eng. Data 2005, 50, 1484.

[106] Tavan, P.; Schulten, K. Phys. Rev. B 1987, 36, 4337.

[107] Englman, R.; Jortner, J. Mol. Phys. 1970, 18, 145.

[108] Ehlers, F.; Lenzer, T.; Oum, K. J. Phys. Chem. B 2008, 112, 16690. 
[109] Zigmantas, D.; Polívka, T.; Hiller, R. G.; Yartsev, A.; Sundström, V. J. Phys. Chem. A 2001, 105, 10296.

[110] Sajadi, M.; Obernhuber, T.; Kovalenko, S. A.; Mosquera, M.; Ernsting, N. P. J. Phys. Chem. A 2009, 113, 44.

[111] Kobrak, M. N. The Chemical Enviroment of Ionic Liquids: Links between Liquid Structure, Dynamics and Solvation; Advances in Chemical Physics, Volume 139, John Wiley \& Sons, Inc., 2008.

[112] Kobrak, M. N. J. Chem. Phys. 2006, 125, 064502.

[113] Arzhantsev, S.; Jin, H.; Baker, G. A.; Maroncelli, M. J. Phys. Chem. B 2007, 111, 4978.

[114] Turton, D. A.; Hunger, J.; Stoppa, A.; Hefter, G.; Thoman, A.; Walther, M.; Buchner, R.; Wynne, K. J. Am. Chem. Soc. 2009, 131, 11140.

[115] Wakai, C.; Oleinikova, A.; Ott, M.; Weingärtner, H. J. Phys. Chem. B 2005, 109, 17028.

[116] Weingärtner, H. Z. Phys. Chem. 2006, 220, 1395.

[117] Weingärtner, H.; Sasisanker, P.; Daguenet, C.; Dyson, P. J.; Krossing, I.; Slattery, J. M.; Schubert, T. J. Phys. Chem. B 2007, 111, 4775.

[118] Hunger, J.; Stoppa, A.; Buchner, R.; Hefter, G. J. Phys. Chem. B 2009, 113, 9527.

[119] Li, H.; Maroncelli, M. J. Phys. Chem. B 2006, 110, 21189.

[120] Li, H.; Arzhantsev, S.; Maroncelli, M. J. Phys. Chem. B 2007, 111, 3208.

[121] Swalina, C.; Arzhantsev, S.; Li, H.; Maroncelli, M. J. Phys. Chem. B 2008, 112, 14959.

[122] Nagae, H.; Kuki, M.; Cogdell, R. J.; Koyama, J. J. Chem. Phys. 1994, 101, 6750.

[123] Andersson, P. O.; Gillbro, T.; Ferguson, L.; Cogdell, R. J. Photochem. Photobio. 1991, 54, 353.

[124] Jin, H.; Baker, G. A.; Arzhantsev, S.; Dong, J.; Maroncelli, M. J. Phys. Chem. B 2007, $111,7291$.

[125] Kuang, D.; Uchida, S.; Humphry-Baker, R.; Zakeeruddin, S. M.; Grätzel, M. Angew. Chem. Int. Ed. 2008, 120, 1949.

[126] Ogura, R.; Nakane, S.; Morooka, M.; Orishashi, M.; Suzuki, Y.; Noda, K. Appl. Phys. Lett. 2009, 94, 073308.

[127] Grätzel, M. Inorg. Chem. 2005, 44, 6841.

[128] Kuhnt, J. Ultraschnelle Dynamik angeregter Zustände von Solarzellen-Farbstoffen; Bachelorarbeit, Göttingen, 2010.

[129] Kuang, D.; Ito, S.; Wenger, B.; Klein, C.; Moser, J. E.; Humphry-Baker, R.; Zakeeruddin, S. M.; Grätzel, M. J. Am. Chem. Soc. 2006, 128, 4146. 
[130] Kuang, D.; Wang, P.; Ito, S.; Zakeeruddin, S. M.; Grätzel, M. J. Am. Chem. Soc. 2006, 128,7732 .

[131] LeBahers, T.; Pauporté, T.; Scalmani, G.; Adamo, C.; Ciofini, I. Phys. Chem. Chem. Phys. 2009, 11, 11276.

[132] Dentani, T.; Kubota, Y.; Funabiki, K.; Jin, J.; Yoshida, T.; Minoura, H.; Miura, H. New J. Chem. 2009, 33, 93. 



\section{Lebenslauf}

Am 26. Juli 1981 wurde ich, Peter William Lohse, als Sohn von Peter Lohse und seiner Ehefrau Claudia Lohse, geb. Heese, in Northeim geboren.

1988 wurde ich in der Grundschule Albanischule Göttingen eingeschult, welche ich bis 1989 besuchte. In diesem Jahr wechselte ich zur Grundschule Tönisvorst bei Krefeld. Im Jahr 1992 wurde ich an der Realschule Tönisvorst aufgenommen welche ich bis 1998 besuchte. Im selben Jahr wechselte ich zum Micheal-Ende-Gymnasium in Tönisvorst um dort im Juni 2001 meine allgemeine Hochschulreife zu erwerben.

Den Zivildienst habe ich von September 2001 bis Juni 2002 in einem Altenpflegeheim (Gösta-Blomberg-Haus) der Heilsarmee geleistet.

Zum Wintersemester 2002/2003 immatrikulierte ich mich an der Georg-August-Universität Göttingen in den Diplomstudiengang Chemie und bestand am 27. Oktober 2004 die Diplomvorprüfung. Im Wintersemester 2006/2007 absolvierte ich ein Forschungspraktikum in der organischen Chemie an der Universität Florenz unter der Leitung von Prof. Dr. Alberto Brandi. Im Juli 2007 begann ich unter der Leitung von Prof. Dr. Jürgen Troe meine Diplomarbeit am Institut für physikalische Chemie der Universität Göttingen und am Max-Planck-Institut für biophysikalische Chemie mit dem Thema „Laserspektroskopische Untersuchungen großer organischer Moleküle“, die ich am 27. Februar 2008 mit der Diplomprüfung abschloss.

Im Anschluss beschäftigte ich mich am Max-Planck-Institut für biophysikalische Chemie (Karl-Freidrich-Bonhoeffer-Institut) in Göttingen und am Institut für physikalische Chemie der Georg-August-Universität Göttingen als wissenschaftlicher Mitarbeiter in der Abteilung von Prof. Dr. J. Troe mit laserspektroskopischen Untersuchungen von ionischen Flüssigkeiten. Das Ergebnis ist diese vorliegende Dissertation. 